6. Hider 
<smiles>CC1CCCCC1C</smiles> 




\section{THESES}

PRÉSENTÉES

\section{A LA FACULTÉ DES SCIENCES DE L'UNIVERSITÉ DE PARIS}

POUR OBTENIR

LE GRADE DE DOCTEUR ESS SCIENCES NATURELLES

PAR

M. EDMOND HESSE

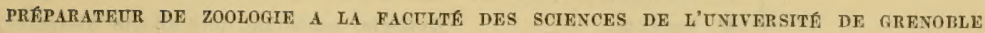

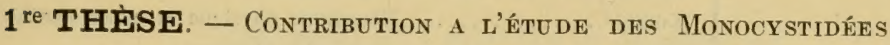
DES OLIGochèTES.

$2^{\text {me }}$ THÈSE. - Propositions données par la Faculté.

Soutenues le 20 , devant la Commission d'examen

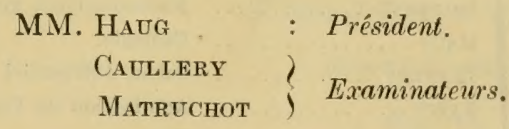

\section{PARIS}

ÉDITION DES ARCHIVES DE ZOOLOGIE EXPÉRIMENTALE

LIBR AIRIE A. SCHULZ

3, Place de la Sorbonne, 3 


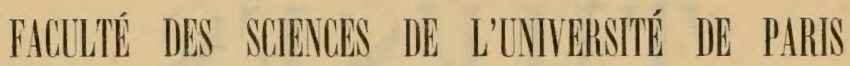

MM.

Doyen..$\ldots \ldots \ldots \ldots$

Doyen honoraire
P. Appeli, Professeur.... Mécanique rationnelle.

G. DaRbodx, - ... Géométrie supérieure.

Professeurs honoraires.... $\left\{\begin{array}{l}\text { L. Troost. } \\ \text { CH. WoLf. } \\ \text { J. RIBAN. }\end{array}\right.$

LIPPMANN

BoutY

Boussinese.

PICARD

H. Porroupf.

Y. DELAGE.............

G. BonNiER

DASTRE.

KCENIGS.

VELAIN

GoURSAT.

Chatin .

PELLAT

HALLER

JoAnnis.

Professeurs

Professeurs-adjoints
JANET

WALLERANT

ANDOYER .

PAINLEVE

HaUe

TANNERY.

RAFFY.

Hodssay

H. Le Chatelier.

Gabriel Bertrand

Mme P. CURIF.

CAULLery

Chabrié.

G. URBAIN .

BOREI

N

I

LEDUC

HADAMARD .

MATRUснот.............

MICHEL

G. PRUVOT

A. GUILIET.

A. Gutiris.
Physique.

Physique.

Physique mathémat. et calcul des probabilités. Analyse supérieure et algèbre supérieure. Astronomie mathématique et mécanique céleste. Zoologie, anatomie, physiologie comparée. Botanique.

Physiologie.

Mécanique physique et expérimentale.

Géographie physique.

Calcul différentiel et calcul intégral.

Histologie.

Physique.

Chimie organique.

Chimie (Enseignement P. C. N.).

Physique (Enseignẹment P. C. N.).

Minéralogie.

Astronomie physique.

Mathématiques générales.

- Géologie.

Calcul différentiel et calcul intégral.

Application de l'analyse à la géométrie.

Zoologie.

Chimie.

Chimie biologique.

Physique générale.

Zoologie, Evolution des êtres organisés.

Chimie appliquée.

Chimie.

Théorie des fonctions.

Zoologie, anatomie, physiologie comparée.

Mécanique et astronomie.

Physique.

Calcul différentiel et calcul intégral.

Botanique.

Minéralogie.

Anatomie comparée. 

A Monsieur le Docteur L. LÉGER, Professeur à l'Université de Grenoble.

A Monsieur le Docteur O. DuboscQ,

Professeur à l'Université de Montpelkier.

\section{Témoignage d'affectueuse reconnaissance}

ED. H. 

ARCHIVES DE ZOOLOGIE EXPERIMENTALE ET GENERALE

$5^{\text {e }}$ Série. Tome III, p. 27 à 301 , pl. I et VII.

25 Décenubre 1909

PAR

\section{EDMOND HESSE}

Préparateur de Zoologie à la Faculté des Sciences de l'Université de Grenoble.

\section{TABLE DES MATIÈRES}

INTRODUCTION

Plan du travail. ....................................... 29

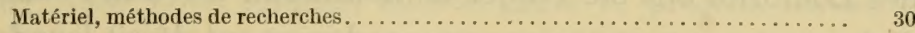

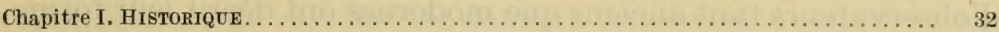

Chapitre II. DESCRIPTION ET ÉTUDE SPECIALE DES DIVERSES ESPECES.

Les parasites et leurs hôtes............................ 40

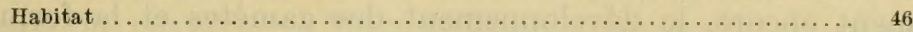

Monocystidées intestinales. Monocystis mitis Leidy ................. 47

Monocystidées de la cavité générale. M. Bretscheri (p. 52), - M. Michaelseni..... 53

Monocystidées génitales : $M$. lumbrici (p. 56), - M, agilis (p. 69), - M. striata (p. 92). - M. hirsuta (p. 97). - M. crenulata (p. 99). - M. Le Mêmei (p. 101). - M. Elmassiani (p. 106), - M. lumbrici olidi (p, 108),-M. villosa (p. 110). - M. turbo (p. 112). - M. hereulea (p. 114). - M. Cogneitii

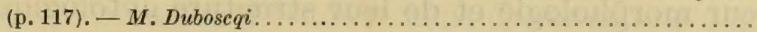

Rhynchocystis pilosa (p. 124), - R. porrecta ................... Nematocystis magna (p. 145), - N. lumbricoides (p, 153), - N. vermicularis (p. 155), $-N$, anguillula . . . . . . . . . . . . . . . . 158

Stomatophora coronata (p. 161). — S. diadema .............. 187

Pleurocystis Cuenoti........................... 189

Zygocystis cometa (p. 194), - Z. pilosa (p. 197). - Z. Legeri........... 199

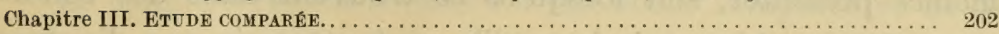

Morphologie. (p. 203). - Cytologie ......................... 206

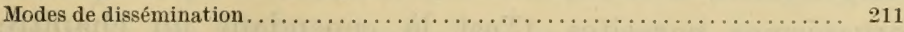

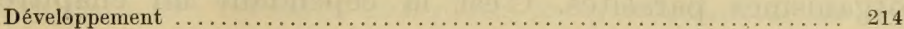

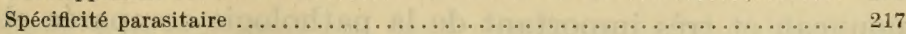

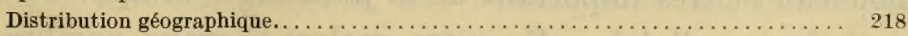

Relation entre le développement des parasites et celui des cellules sexuelles de l'hôte 219

AROH, DE ZOOL. EXP. ET GEX. - 5 SERIE, - T. III. - (II).

3

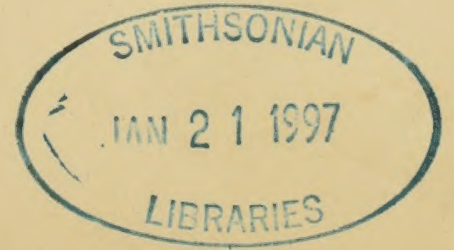


Chapitre IV. ALTRRATIONS ET PARASITES.

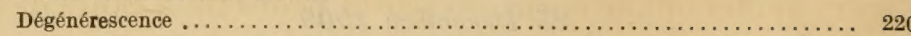

M. agilis (p. 221). - M. lumbrici (p. 223), - R. pilosa (p. 225). - N. vermicularis (p. 227). - St. diadema (p. 227). - M. lumbrici olidi (p. 229). St. coronata (p. 229). - M. Le Mêmei ....................... 232

Parasites . . . . . . . . . . . . . . . . . . . . . . . . . . . . . . . . 233

Chapitre V. AOTION PATHOLOGIQUE SUR L'HÔTE ET REACTIONS DFFENSIVES DE CET HÔTE...... 240

Monocystidées intestinales ............................. 244

Monocystidées cœlomiques .............................. 244

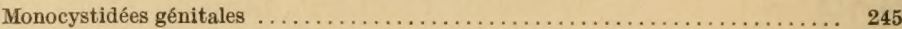

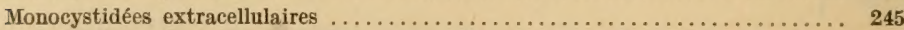

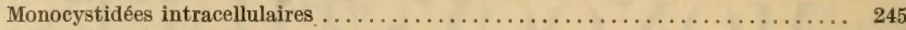

N. magna (p. 245). - Histologie normale des pavillons ciliés (p. 245). - Cel-

lules cupuliformes . ............................. 247

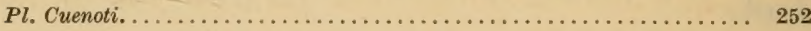

Monocystidees intrablastophoriennes (p. 253). - Action sur le blastophore (p. 254). - Action sur les cellules sexuelles.................... 258

M. agilis (p. 259). - Pénétration précoce (p. 260). - Pénétration tardive.... 264

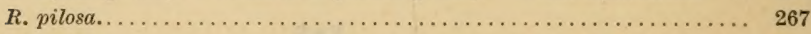

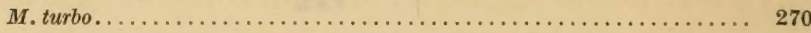

St. coronata. Pénétration précoce (p. 271). - Pénétration tardive ........ 272

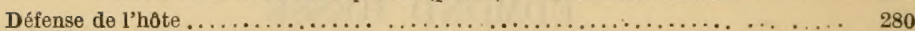

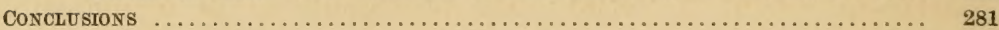

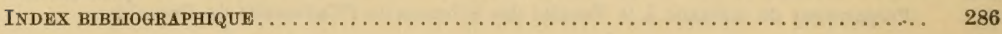

EXPLICATION DES PLANCHES . . . . . . . . . . . . . . .

\section{INTRODUCTION}

Les Monocystidées des Oligochètes, particulièrement celles des Lombrics ont été l'objet de nombreux travaux ; beaucoup d'observateurs tant anciens que modernes ont décrit leur forme, leur structure, leur enkystement, leur sporulation. Tout récemment encore, le développement des gamètes et la formation des sporocystes ont été étudiés avec soin et l'anisogamie nettement mise en évidence. Mais si ces parasites sont bien connus à divers points de vue, on a beaucoup négligé l'étude comparée de leur morphologie et de leur structure cytologique, celle des premières phases de leùr évolution et celle, pourtant si intéressante de l'action qu'ils exercent sur l'organisme de l'hôte, On ne connaît pas non plus les altérations qu'ils peuvent euxmêmes présenter, soit lorsqu'ils se trouvent dans des conditions défavorables, soit lorsqu'ils sont attaqués par d'autres organismes parasites. C'est là cependant un chapitre tout nouveau et très important de la pathologie comparée et de la pathologie cellulaire. En outre, malgré le grand nombre d'au- 
teurs qui les ont étudiées, ou plutôt peut-être à cause de ce fait même, la systématique des Grégarines des Lombriciens est très embrouillée. Dans la plupart des travaux, elles sont désignées communément, sans spécification sous le nom de Nonocystis du Lombric ou encore confondues sous la dénomination de Monocystis agilis Stein. Tombant dans un excès contraire, les chercheurs qui se sont occupés plus particulièrement de leur systématique ont multiplié les espèces, trompés par la grande variété de formes que peut prendre un même parasite.

CuÉnot (1902) a done pu dire avec juste raison " qu'il est presque impossible de se retrouver dans le dédale de leur synonymie et de leur appliquer un nom correct „); il a tenté de mettre un peu d'ordre dans ce chaos; mais, comme il s'attachait plus particulièrement à l'étude des processus de la fécondation, il n'a pas éclairci complètement la question.

Ainsi, au quadruple point de vue de la morphologie et par suite de la systématique, du développement, de la pathologie et de l'action sur l'hôte, les Monocystidées des Oligochètes sont mal connues, il était done important de reprendre leur étude. Nous nous sommes efforcé dans le présent travail de compléter et de rectifier les données imprécises et souvent contradictoires que l'on possède sur tout ce groupe de parasites.

Plan du TRavaIL. - Notre mémoire comprendra six chapitres.

Le chapitre Ier est consacré à l'historique.

Dans le chapitre II, nous décrivons les divers parasites que nous avons eu l'occasion d'étudier, nous donnons une diagnose précise des espèces et nous établissons aussi exactement que possible leur synonymie; une trentaine d'espèces dont 18 nouvelles y sont passées en revue.

Le chapitre III est consacré à une étude comparative de ces diverses espèces ; c'est une sorte de résumé de la biologie générale des parasites où nous indiquons les particularités de leur éthologie, leur morphologie, leur cytologie et leur développement. 
Dans le chapitre IV, nous étudions les diverses altérations présentées par nos Monocystidées, les parasites qu'elles hébergent et les dégénérescences causées par ces parasites.

Dans le chapitre V, nous nous occupons de l'action pathologique exercée sur les divers organes parasités des Oligochètes, ce qui nous conduit à dire quelques mots sur certains points de l'histologie normale de ces organes encore incomplètement connus. Puis nous indiquons succinctement quels sont les divers modes de défense des hôtes. Enfin le chapitre VI renferme les conclusions du travail.

MATÉRIEL et MÉTHOdes DE RECHERChes. - Il a été nécessaire pour nos recherches d'étudier des Oligochètes recueillis en toute saison et à divers stades de leur développement. Nous verrons ainsi que, en ce qui concerne Lumbricus herculeus par exemple, ce sont les individus jeunes, à glandes sexuelles mâles peu développées, en état de prespermatogénèse qui nous ont montré de très nombreux stades jeunes de Monocystis agili.s, tandis que ces stades sont peu abondants dans les Lombrics adultes; ce sont également ces jeunes $L$. herculeus qui donnent asile à $M$. herculea : le parasite ne se rencontre qu'exceptionnellement dans les Lombrics arrivés à la maturité sexuelle.

Les Oligochètes récoltés en diverses régions, nous les avons ouverts et nous avons examiné avec soin l'intestin, la cavité générale et les vésicules séminales. Lorsqu'ils contenaient des parasites, ceux-ci ont été étudiés, d'abord in vivo, dans le liquide physiologique, puis par la méthode des frottis et par celle des coupes sériées. Pour celles-ci, il est souvent nécessaire de débarrasser l'intestin des graviers qu'il contient; dans ce but nous avons employé les méthodes classiques : les animaux mis au jeûne étaient gardés dans des flacons avec du papier buvard humide, ou placés pendant quelque temps dans du marc de café.

Nous avons utilisé comme fixateurs : le sublimé acétique ou physiologique, le sublimé alcool de Schaudinn, l'alcool absolu le Flemming fort, le Flemming-Benda (fixateur de Benda 
pour la recherche des mitochondries), enfin le formol picrique de Bouin modifié par Duboseq et dont Brasil (1905) a indiqué la formule. Le sublimé acétique et le Flemming nous ont donné les résultats les meilleurs.

Comme colorants, nous nous sommes servi de l'Hématoxyline (méthode de Heidenhain à l'alun de fer ou méthode à l'Hémalun de Mayer) suivie d'une coloration cytoplasmique par l'éosine, l'Orange Gou le Vert-lumière (ce dernier colorant surtout nous a été très utile pour mettre en évidence certaines structures); - de la Safranine ou du Rouge magenta (triple coloration de Flemming), ou méthode de Benda (Safranine ou Magenta vert-lumière); - du carmin : picro-carmin, carmin au lithium, picro-indigo-carmin; - le Kristallviolet (méthode de Benda pour la recherche des mitochondries); le Mann (bleu de méthyle-éosine, méthode lente), le Giemsa nous ont donné aussi quelques résultats intéressants.

Nous présentons ici avec plaisir nos meilleurs remerciements à tous ceux qui nous ont aidé au cours de ces recherches. A notre vénéré Maître, M. le professeur Léger qui nous a constamment guidé de ses conseils et dont l'érudition et la longue expérience nous ont été si souvent d'un grand secours, nous sommes heureux d'adresser l'hommage de notre plus vive gratitude.

M. le professeur Duboscq nous a donné des renseignements techniques et bibliographiques; il a daigné revoir quelques unes de nos préparations et nous a facilité l'interprétation de quelques points délicats; nous l'en remercions bierı sincèrement.

Nous avons mis quelquefois à contribution les connaissances taxonomiques de M. le professeur Michælsen de Hambourg et de M. Karl Bretscher, nous les prions de vouloir bien agréer l'hommage de nos remerciements.

Nous n'aurons garde d'oublier l'extrême obligeance de M. Cognetti de Martiis, du musée de Turin qui nous a déterminé de nombreux échantillons d'Oligochètes. Son amabilité lui donne droit à toute notre reconnaissance. 
Qu'il nous soit permis enfin de remercier également ici M. le professeur Pruvot, notre premier maître en zoologie et M. Racovitza directeurs des Archives de zoologie expérimentale, pour l'honneur qu'ils nous ont fait en acceptant ce travail dans leur périodique.

\section{CHAPITRE PREMIER}

\section{HISTORIQUE}

Des Grégarines des Oligochètes, la plus anciennement connue est celle du Lombric.

La découverte de ces Grégarines du Lombric est généralement attribuée à DUJARDIN (1835); cet observateur en effet a décrit le premier une espèce vivant dans le cœlome d'un Ver de terre; il en a décrit les mouvements caractéristiques, mais il confond ce parasite avec le Proteus tenax O.-F. Müller.

SurIRAy (1836) déclare avoir fait connaître antérieurement cet organisme dans un mémoire communiqué à la Société Linnéenne de Normandie, et reconnaît que ce n'est pas un vrai Protée, il le nomme "Sablier protéiforme ».

Longtemps avant ces savants, d'autres naturalistes avaient observé le même parasite enkysté dans les vésicules séminales du Ver de terre; mais sans comprendre la véritable nature de ces kystes. C'est ainsi que Home (1823) et Montègre (1823) étudiant l'anatomie du Lombric terrestre prennent ces kystes pour les œufs de l'hôte et considèrent comme ovaires les vésicules séminales dans lesquelles ils les ont rencontrés.

Morren (1826) commet la même erreur, et, retrouvant ces orgnnismes inclus dans des phagocytes, à l'extrémité du corps du Ver parasité, il les considère comme des œufs libres qui auraient été transportés à l'extrémité du corps.

DuGìs (1828) regarde également les kystes de Monocystis comme des œufs de Lombric, du moins ceux qu'il rencontre 
dans les vésicules séminales (les ovaires, pour lui); quant à ceux qui se trouvent dans la cavité générale, ce sont pour lui des échinocoques.

Henle (1835), reconnaît la nature parasitaire de ces formations ; il compare la forme des spores à des graines de courge et les considère comme des "Navicules ".

Hoffmeister (1842) regarde également les spores comme des "Navicules ".

Mecket (1844) reprenant pour son compte l'erreur des anciens observateurs décrit à nouveau les kystes parasitaires comme œufs du Lombric.

Dujardin (1845) homologue les spores aux "Psorospermies " décrites par Müller chez les Poissons.

Cette même année 1845, HENLE décrit dans les vésicules séminales du Lombric une Grégarine qu'il nomme Gregarina lumbrici; deux autres espèces sont également vues par cet observateur, dont l'une est probablement celle que Schmidt a appelée plus tard Monocystis magna; mais elles sont trop mal figurées et insuffisamment décrites pour être reconnaissables. L'auteur croit à une relation entre les Grégarines et les “ Navicules » qu'il a décrites antérieurement, mais il ne sait pas exactement laquelle.

STEIN (1848) trouve et décrit Zygocystis cometa; il a suivi avec soin la formation des kystes et des spores chez cette espèce et démontré ainsi l'erreur de MEcket. La relation qui existe entre les Grégarines et les "Navicelles » est ainsi nettement mise en évidence. Dans ce même travail, Stein donne des dessins, malheureusement un peu trop schématiques, d'une autre Grégarine qu'il nomme Monocystis agilis.

Frantzius (1848) observe des kystes où il n'y a qu'un seul noyau.

BRUCH (1849) pense que Gregarina lumbrici " hervorbildet" des larves de Nématodes du Lombric et que ce n'est pas autre chose que "stillgewordene Filaria".

Schmid (1854) distingue trois espèces dans $L$. agricola : 
Monocystis magna, $M$. cristata dont il constate la grande ressemblance avec $\boldsymbol{M}$. lumbrici Henle ; et une autre espèce qu'il désigne comme $M$. agilis Stein, remarquable par l'extrême variabilité de sa forme. Il décrit également une espèce parasite de $L$. rubellus, $M$. porrecta qu'il a rencontrée aussi mais rarement dans $L$. agricola; cette Grégarine rappelle beaucoup M. magna, mais l'auteur ne l'a jamais observée comme cette dernière dans les "Nebenhoden". Chez L. olidus, Schumd rencontre encore une autre espèce, arrondie et couverte de poils courts.

Cet auteur a reconnu la nature des poils qui recouvrent certaines Grégarines et montré que ce sont là des spermatozoïdes de l'hôte altérés. Enfin, il a étudié divers stades jeunes du parasite dans les blastophores de l'hôte; mais il prétend que jamais deux individus ne se rassemblent pour former un kyste. Ces kystes ne renfermeraient qu'un seul individu, parfois même un simple fragment d'individu.

LIEBERKÜHN (1855) étudie surtout l'évolution des parasites. Il pense que les "psorospermies " (sporocystes) donnent naissance à des Amibes qui deviennent des Grégarines; celles-ci donneraient les psorospermies par division. Il constate la présence de poils, tantôt cunéiformes, tantôt en forme de cils fins et observe que les "Grégarines velues sont sujettes à la mue ». Il représente des $M$. magna, $M$. agilis, Zygocystis cometa, et une Grégarine arrondie, poilue, analogue à $M$. lumbrici olidi Schmidt ; d'autres formes arrondies dessinées par Lieberkühn sont probablement pathologiques.

D'Udenem (1856) suit le développement des psorospermies qu'il considère comme des parasites spéciaux et, découvrant les véritables ovaires du Lombric, démontre l'erreur des auteurs qui ont pris les kystes grégariniens des vésicules séminales pour des œufs de l'hôte. Il décrit et représente des parasites filamenteux trouvés dans les entonnoirs vibratils et que Stein (1842) avait regardés comme les ovaires du Lumbricus. Il s'agit là, vraisemblablement d'après description et figures 
de $M$. magna Schmidt, bien que d'Udeкem leur attribue une " absence complète de mouvement ".

LANKESTER (1863) fait un historique succinct des Monocystis parasites du Lombric; il décrit des $M$. lumbrici sans noyaux et des enkystements solitaires.

SCHNEIDER (1875) combat longuement les conclusions de LIEBERKüHN sur l'évolution des Monocystis; il trouve dans les spores les " corpuscules falciformes " qu'il considère comme les germes des Grégarines, il constate l'existence de macro et de microspores très polymorphes et dont il représente les diverses formes. Il ne croit pas à la réalité des mues signalées par Lieberkühn, mais ne sait comment expliquer ce phénomène de rejet de la cuticule très bien compris cependant auparavant par SсHмIDT.

Gabriel $(1876,1877,1878,1879,1880)$, de même que LieberKÜHN croit que les spores des Monocystis se développent sur place dans les testicules du Lombric et donnent d'abord des Amibes ; celles-ci se rassemblent en "Synamöben" d'où proviendraient les Grégarines. L'auteur conclut de ce fait à la parenté des Grégarines et des Myxomycètes.

BüTschli (1881) trouve en grande quantité $M$. magna et décrit son mode d'attache aux cellules épithéliales vibratiles des pavillons séminaux, il donne une bonne description du sporocyste et des huit sporozoïtes nucléés qu'il renferme.

En 1882, dans son traité classique (Bronn's Thierrheich) il expose l'état des connaissances sur les Monocystis du Lombric qu'il appelle tantôt $M$. agilis, tantôt $M$. tenax. Il partage l'avis de Schмid sur les poils qui recouvrent cette espèce. En ce qui concerne le développement, la présence de corpuscules falciformes dans les spores lui fait rejeter les assertions de Gabriel et de LieberküHN.

BaLBIANI (1884) écrit également quelques mots sur l'historique des Monocystis du Lombric et attribue par erreur à Léon Dufour au lieu de DuJardin la découverte de la Grégarine cœlomique du Ver de terre. 
Ruschinaupt (1885) décrit les stries cuticulaires longitudinales de $M$. magna ; il observe l'aspect filiforme des poils antérieurs de $1 I$. cristata Schmidt et distingue dans le Lombric plusieurs espèces nouvelles: $\boldsymbol{M}$. cuneiformis, $\boldsymbol{M}$. minuta. Il admet l'enkystement solitaire suivi d'une division à l'intérieur du kyste. Pour lui, le nucléus de reliquat visible dans les sporocystes serait le vrai germe des Grégarines et non les corpuscules falciformes comme le pensent SCHNEIDER et BÜTSCHLI. Ruschinupt croit avoir vu la spore pénétrer dans les "Spermamutterzellen " de l'hôte et s'y développer en Grégarine ; les figures qu'il donne des stades jeunes intracellulaires du parasite rappellent celles de Sснмпт sur le même objet. On trouve aussi dans ce travail de RuschHaupt quelques observations sur la fréquence relative des Grégarines et des kystes aux diverses saisons de l'année.

Hennegur (1888) étudie en détail la formation des spores et constate que la multiplication des noyaux se fait par mitose dans les kystes; mais il n'a pas remarqué que la sporulation était précédée de l'accolement de deux individus.

Pfeiffer (1891 et 1893) représente des Monocystis pilosa (qu'il appelle $M$. magna), dont la tête est piquée dans des blastophores flétris qu'il considère comme des restes de "Becherzelle». Il croit à un enkystement solitaire et à la division ultérieure des parasites dans le kyste. Il a rencontré des sporocystes mûrs de Monocystis dans l'intestin des Bécasses qui se nourrissent de Vers de terre. Il donne, de même que RusCHHAUPT quelques détails sur les relations qui existent entre le développement du parasite et les diverses saisons.

WoLTERS'(1891) a constaté nettement qu'il y a accolement de deux individus pour former un kyste, mais il croit avoir vu une expulsion de globules polaires et une karyogamie, faits qui n'ont pas été confirmés par les observations récentes.

BoSANQUet (1894) décrit une Grégarine cœlomique arrondie de $L$. herculeus, parfois libre dans le cœlome, parfois enfermée 
dans les cellules situées autour de l'intestin. Il a vu également les kystes de ce parasite.

ClARKE (1895) croit pouvoir confirmer les observations de Wolters.

LABBÉ (1899) donne une diagnose de toutes les espèces décrites jusqu'à lui ; mais, comme le lui reproche arec juste raison Cuénot, il ne les a pas étudiées lui-même « et délimite les formes au petit bonheur ".

CÚ́Not (1901) étudie soigneusement les processus de fécondation chez les diverses Monocystis des Lumbricus et Allobophora et, en même temps, essaie de caractériser avec un peu plus de précision que ses devanciers les Grégarines adultes, sans cependant réussir complètement dans sa tentative, comme nous le verrons.

Cecconr (1902) observe dans Allobophora complanata Dugès un parasite qu'il identifie à Monocystis agilis Stein, et constate un enkystement solitaire, suivi d'une conjugaison des kystes deux à deux.

Prowazek (1902) suit les processus de la formation des spores et de la différenciation des sporozoïtes dans leur intérieur.

Drzewecki (1903) découvre de curieuses transformations dans le noyau, au cours de la vie végétative, chez $M$. agilis et $M$. porrecta; il a cru voir les sporozoïtes au moment de leur entrée dans les blastophores; mais les figures qu'il donne des premiers stades du développement ne montrent pas trace de parasite.

Plus tard, le même observateur (1907 a) décrit deux espèces nouvelles ( $M$. ciliata et Stomatophora borealis) parasites de Lombrics de Saint-Pétersbourg et d'Arkangel.

BRASIL (1905) décrit et étudie en détail les premiers phénomènes de la division nucléaire chez les parasites enkystés et il met en évidence une anisogamie peu marquée.

Hoffmann (1908) trouve au contraire un anisogomie très accentuée chez une Monocystis de $L$. herculeus. 
Telles sont, brièvement résumées les diverses observations relatives aux Monocystis des Lombriciens. Il nous reste à énumérer les recherches concernant les Grégarines des autres Oligochètes; elles sont beaucoup moins nombreuses.

Menge (1845) observe dans le cœlome de Euaxes obtusirostris des corps en croissants et des capsules visibles à travers les téguments et qui ne sont probablement pas autre chose que des Monocystis cœlomiques avec leurs kystes.

KöLliKer (1848) décrit Gregarina enchytraei de l'intestin d'Enchytraeus albidus Henle, et $G r$. Scenuridis dans les parties sexuelles mâles des plus grands individus de Saenuris variegatus Hoffm.

Claparìde (1861) observe une Grégarine immobile dans l'intestin de Pachydrilus semifuscus Clap.

RadKewitsch (1870) publie en russe un travail sur Monocystis enchytraei Kölliker.

RAY Lankester (1872) signale dans Tubifex rivulorum une Monocystis dont les sporocystes présentent un appendice caudal filamenteux et qui, depuis a été, à tort, croyons-nous, homologuée avec $M$. saenuridis Kölliker.

VeJdowsky (1876) signale des Monocystis dans la cavité générale et les testicules de divers Oligochètes (Psammoryctes umbellifer, Rhynchelmis limosella, Phreatothrix).

Perrier (1881) rencontre dans la cavité générale des Pontodrilus diverses Grégarines monocystidées dont quelques-unes possèdent un appareil fixateur assez compliqué.

LeIdy (1882) remarque dans l'intestin de Distichopus silvestris de petites Grégarines, Monocystis mitis qu'il considère comme très proches de $\boldsymbol{M}$. enchytraei Kölliker et dans lesquelles, il a remarqué de petits corps elliptiques qui seraient pour lui les spores du parasite et qui sont probablement des organismes voisins des Metchnikovella.

NASSE (1882) a observé autour des kystes de Urospora Saenuridis une couverture de cils qui sont probablement des spermatozoïdes altérés de l'hôte, comme le suppose Bütschli. 
Beddart (1888 et 1889) trouve dans Perichaeta novaezelandiae et Megascolex armatus, deux Monocystis dont il ne connaît pas les spores.

EIsen (1895) décrit deux espèces : Spermatobium Freudi et $S p$. eclipidrili, qui paraissent être des Monocystis typiques, bien que l'auteur les considère comme intermédiaires entre les Klossia et les Monocystis. Les stades jeunes seraient parasites dans les spermatogonies et non dans les blastophores de l'hôte. Il y aurait une schizogonie avec multiplication du noyau par mitose, et, pour la formation des sporocystes, le noyau se multiplierait par division multiple.

GIARD (1898) signale sans les décrire des Grégarines parasites dans un Oligochète exotique acclimaté à Wimereux (Microscolex phosphoreus Dugès).

Mrazek (1899) publie une note préliminaire sur une Monocystis de Rhynchelmis; il a suivi la sporulation et observé la division des noyaux par mitose.

Nusbaum (1903) fait connaître une espèce nouvelle, Schaudinnella henleae, de l'intestin de Henlea leptodera Vejd., remarquable par les processus très spéciaux de sa sporulation.

Nous avons nous-même donné (1904) une brève diagnose d'une Grégarine parasite des Pheretima.

Drzewecki (1908) a repris l'étude de cette espèce et $€$ n a fait, comme nous le verrons plus loin, le type d'un genre nouveau.

Mentionnons enfin les traités classiques de Bütschli (1882) Delage et Hérouard (1893), Wasielewsky (1896), Labbé (1899), Doflein (1901), Minchin (1903), Lühe (1904) où sont énumérées ou étudiées diverses espèces.

L'histoire des Monocystis des Oligochètes étant ainsi rapidement esquissée, avant de commencer leur étude, il nous reste à indiquer quelques caractères qui leur sont communs et à dire quelques mots de leurs hôtes. 


\section{DESCRIPTION ET ÉTUDE SPÉCIALE DES ESPÈCES}

\section{Les Parasites et leurs Hôtes.}

Les Grégarines que nous allons étudier appartiennent toutes au sous-ordre des EugrÉGARINES (Léger) et à la tribu des Acephalina (Kölliker) =Monocystidea (Stein). Elles sont caractérisées ainsi qu'il suit dans les traités classiques :

Eugrégarines dans lesquelles le corps ne présente pas de septum, ni d'épimérite à aucun stade. Parasites surtout colomiques. Spores biconiques à pôles semblables.

Nous verrons que nos parasites ne possèdent pas de septum et qu'ils ont tous des spores biconiques à pôles semblables, mais quelques-uns d'entr'eux présentent une différenciation de la portion antérieure du corps, véritable appareil épiméritique pouvant servir à les fixer à une cellule hôte, parfois aussi compliqué que les vrais épimérites des Polycystidées et, comme eux transitoire. Le titre de Monocystidea leur convient donc mieux que celui de Acephalina.

On sait que la plupart des espèces de Monocystidées des Oligochètes sont des Grégarines cœlomiques et que deux seulement ont été rencontrées dans l'intestin. Pour être complet, nous dirons quelques mots de l'une de ces dernières que nous avons eu l'occasion d'observer chez Fridericia polycheta Bretsch.

Quelques-unes de ces espèces se rencontrent uniquement dans la cavité générale, entre l'intestin et la paroi du corps, principalement dans les derniers segments; d'autres se rencontrent à la fois dans le colome proprement dit et dans les vésicules séminales qui n'en sont qu'une dépendance, d'autres enfin ne vivent que dans les vésicules séminales, parfois même dans une région bien localisée de celles-ci.

Un certain nombre de ces formes sont nouvelles et ne nous paraissent pas avoir été figurées; la plus grande partie ont été souvent observées et décrites; mais les descriptions qu'on en a données ne sont pas toujours très exactes et ne concordent 
pas entr'elles, de sorte que leur synonymie est assez embrouillée; elle est rendue plus difficile encore par ce fait que les auteurs n'ont pas toujours désigné exactement les hôtes; la synonymie de ces hôtes eux-mêmes est bien compliquée, à tel point que l'on pourrait dire que rien n'est plus fastidieux que la synonymie des Monocystis des Lombrics, si ce n'est la synonymie de leurs hôtes.

Nous donnons ci-après un tableau des hôtes étudiés nommés d'après Michaelsen (1900) avec leur synonymie; en face de chacun de ces hôtes se trouve la liste des Monocystis qu'il renferme avec leur synonymie et le nom des organes parasités.

LUMBRICUS TERRESTRIS L., Mull. = Enterion herculeum Savigny.

$=L$. herculeus Duges

$=L$. agricola Hoffmeister.

L. RUBELLUS Hoffm.

= Enterion rubellum Örley

L. CASTANEUS Sav.

$=L$. purpureus Eisen

$=\boldsymbol{L}$. minor Johnston

= Enterion pumilum Sav.

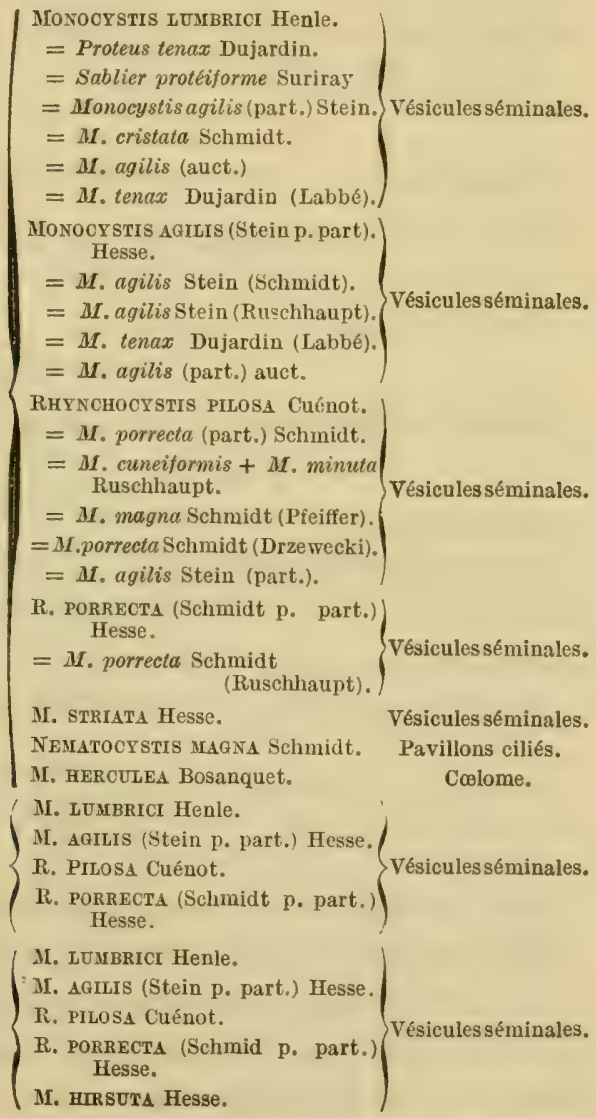




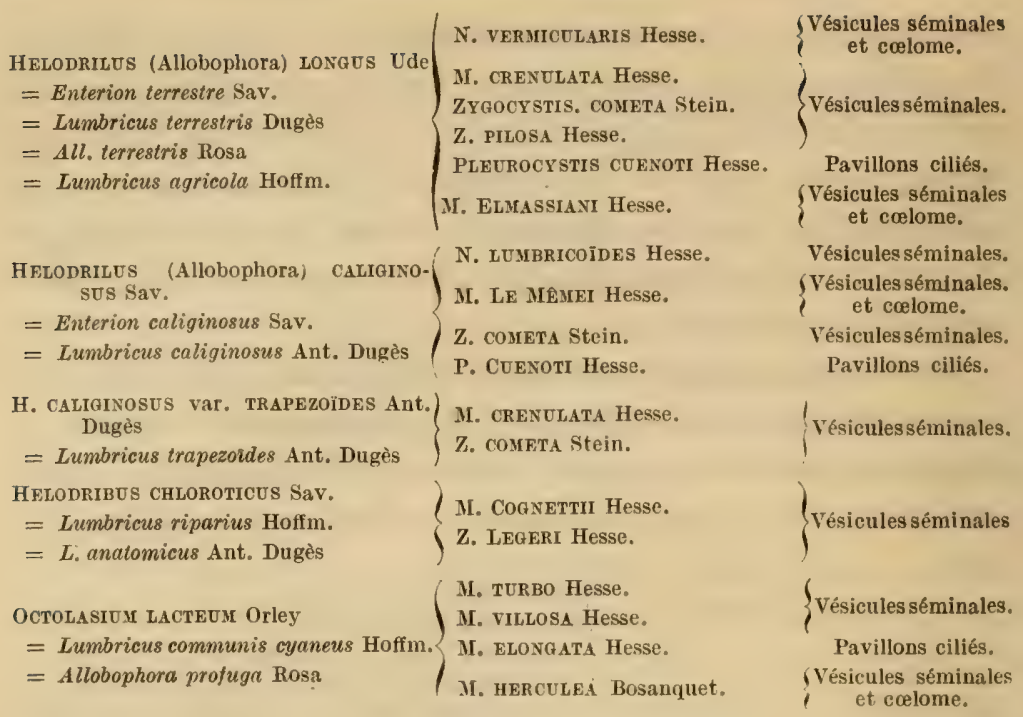

EISENIA FGETIDA Sav.

\} M. LUмвRici ouid Schmidt. Vésicules séminales.

$=L$, olidus Hoffm.

M. Elmassiani Hesse.

II. LTMRRICI OLIDI Schmidt.

\}ésicules séminales. Eisen.

$=L$. puter Hoffm

Jamais de Grégarines.

EISENIELLA TETRAEDRA Sav.

Pheretma rodericensis Grube $=$ Perichoeta rodericensis Grube

Pheretima hawayana Rosa $=$ Perichoeta aspergillum Beddart

= Perichoeta bermudensis Beddart

LUMBRICULUS VARIEGATUS MULI.

= Lumbricus variegatus Mull.

= Saenuris variegatus Johnston

FRIDERICIA POLYChETA Bretscher

DISTICHOPUS SYLVESTRIS Leidy

(Stomatophora coronata Hesse.

$\int$ N. ANGUILLULA Hesse.

II. MaCrospora ilesse (kystes et spores).

Coclome.
S. coronata Hesse.
S. DIADEma Hesse.
N. ANGUILLULA Hesse.
M. Mrichaelseni Hesse.
II. MACrospora Hesse (kystes et spores).
¿Vésicules séminales.
Cœlome.

$\{$ М. Duвоscqi Hesse.

¿Vésicules séminales

\{ M. Bretscheri Hesse. Cœlome.

M. Mris Leidy. Intestin.

I. MrTs Leidy. Intestin.

Pour compléter la nomenclature des Monocystis des Oligochètes, nous indiquons dans le tableau suivant les espèces que nous n'avons pas observées. L'ensemble des deux tableaux donnera ainsi une liste complète des Monocystidées jusqu'ici étudiées chez les Oligochètes. 
DIPOROCHAETA INTERMEDIA (Beddart) = Perichaeta novae-zelandice (Beddart)

M. PERICHAETAE Beddart.

Vésicules séminales.

MEgASCOLEX MLAURTII (Kinb.)

$=$ Perichaeta armata (Beddart)

$=$ Megascolex armatus (Rosa)

IUMBriculus VARIEGatús Mïll

$=$ Lumbricus variegatus Müll

= Saenuris variegata Jolınston

ECLIPIDRHuS FRIGIDTS Eisen

OCNERODRIUUS TASTE Eisen

$=$ Phonicodrilus taste Eisen

Henled NASUTA Eisen

$=\boldsymbol{H}$. leptodera Vejd.

ENch YTRAEUS ALBIDUS Henle

Fridericta GaLBa Hoffm.

= Enchytraeus galba Hoffm.

Fridericta Hegemon Vejd.

= Enchytraeus Hegemon Vejd.

JUMBricillus PAGENSTECHERI Ratz.

$=$ Pachydrilus pagenstecheri Vejd.

Marionta semifusca Clap.

$=$ Pachydrilus semifuscus Clap.

TUBIFEX TUBIFEX Müll.

= Lumbricus tubifex Müll.

= Tubifex rivulorum Lamarck

= Saenuris variegata Hoffm.

$$
\}
$$

M. PERtchatan Beddart. Vésicules séminales.

II. sıtexuribrs Kölliker. (Vésicules séminales

$\int$ t et cavité générale.

$\left\{\begin{array}{c}\text { Spermatobium ECLIPIDRILI Eisen. } \\ =\text { Spermatophagus (Labbé)ecli- } \\ \text { pidrili Eisen. }\end{array}\right.$

\} SPERJLATOBIUM FREUdI Eisen. Vésicules séminales

\}=Spermatophagus freudi Eisen.

SCHAUDINNELLA HENLEAE, Nus-
baum.

Mr. ENCHYTRAeI Kölliker. $\quad\left\{\begin{array}{c}\text { Vésicules séminales } \\ \text { et cavité générale. }\end{array}\right.$

f et cavité générale.

M. РАCH Y DRILI Clap. Vésicules séminales.

\section{Si l'on metà part le genre aberrant Schaudinnella Nusbaum,} si différent, d'après les données de l'auteur, de toutes les Grégarines connues actuellement, par les processus spéciaux de sa reproduction sexuée et par les caractères de ses sporocystes et de ses sporozoïtes, les autres Monocystidées des Oligochètes constituent un groupe assez homogène. Seul le genre Urospora A. Schneider se distingue par l'aspect de ses sporocystes pour-

(1) Cette espèce est désignée par tous les auteurs sous le nom de Urospora saenuridis Kölliker ; mais elle ne peut pas porter ce nom. L'espèce décrite par KöLLIKER (1848) est en effet une Monocystis et non un Urospora, il suffit pour s'en convaincre de jeter un coup d'œil sur la fig. 22, Taf. 11 et la tlgure 28, Taf. 3 où Kölliker a représenté les kystes et les sporocystes de ce parasite, ceux ci sont dépourvus du filament caudal caractéristique des sporocystes du genre Urospora. L'espèce trouvée par RAY LANKESTER (1872) dans Tubifex tubifex Irüller, a des sporocystes appendiculés, c'est donc un Urospora à laquelle il faut donner un nom nouveau, nous proposons celui de tubificis. Urospora saenuridis Kölliker, de Ray Lankester devient ainsi Urospora tubificis nom. nov. La confusion provient sans doute de ce que Kölliker a mal identifié l'hôte, il le nomme en effet : Saenuris variegata Hoffm., mais il indique comme synonyme Lumbricus variegatus O. Müll., or d'après BEDdART (1895) et MrchaELSEN (1900), Lumbricus variegatus Müller = Lumbriculus variegatus Müll. tandis que Saenuris variegate Hoffm. = Tubifex tubifex Mü̈ll. 
vus d'un filament caudal, les autres parasites présentent tous des sporocystes biconiques à pôles semblables, dépourvus d'appendice. Ce caractère a conduit les auteurs à ranger tous ces parasites dans un seul genre, le genre Monocystis, malgré la grande diversité d'aspect des états végétatifs. Mais cette diversité est si considérable que le genre Monocystis constitue un ensemble fort disparate au point de vue morphologique. Il nous paraît nécessaire d'y faire des coupures et de créer des groupements homogènes de façon à établir un peu d'ordre dans ce chaos. Nous ne faisons du reste que suivre l'exemple donné pour d'autres familles de Grégarines, c'est ainsi que l'on s'est basé également sur les caractères morphologiques du trophozoïte pour grouper en genres un certain nombre d'ActinocéPHALIdes à sporocystes très voisins. Chez les Monocystidées même, Stein a déjà créé le genre Zygocystis pour des espèces que l'on rencontre constamment en association; chez ces parasites, l'accolement se fait par les extrémités céphaliques ; d'autres espèces sont néogames comme les Zygocystis, mais avec un accolement latéral et longitudinal semblable à celui que l'on observe chez les Pleurozyga, nous les rangerons dans le genre Pleurocystis. Il existe en outre des parasites sphériques, ovoïdes, peu allongés, tandis que d'autres sont longs, cylindriques et nématoïdes, nous créons pour ceux-ci le genre Nematocystis, les premiers restant des Monocystis. A côté de ces espèces dépourvues d'appareil fixateur nettement différencié, d'autres possèdent un véritable épimórite, tantôt en forme de trompe cylindro-conique plus ou moins allongée, ils rentreront dans le genre Rhynchocystis, tantôt en forme de ventouse, ce seront les Stomatophora.

De la sorte, les Monocystidées des Oligochètes se trouvent divisées en huit genres :

Genre Monocystrs Stein. Corps ovoïde ou sphérique sans différenciation antérieure bien marquée. Trophozoütes solitaires. Sporocystes biconiques à pôles semblables non appendiculés (fig. I). 
G. Nenatocystis g. nov. Corps cylindrique allongé, de grande taille. Aspect nématoüde. Trophozoïtes solitaires. Sporocystes biconiques à pôles semblables non appendiculés (fig. II).

G. Rhynchocystis g. nov. Corps ovoïde ou cylindroide. Pôle antérieur muni d'un épimérite métabolique le plus souvent allongé en une trompe conique ou cylindro-conique. Sporocystes biconiques à pôles semblables non appendiculés (fig. III).

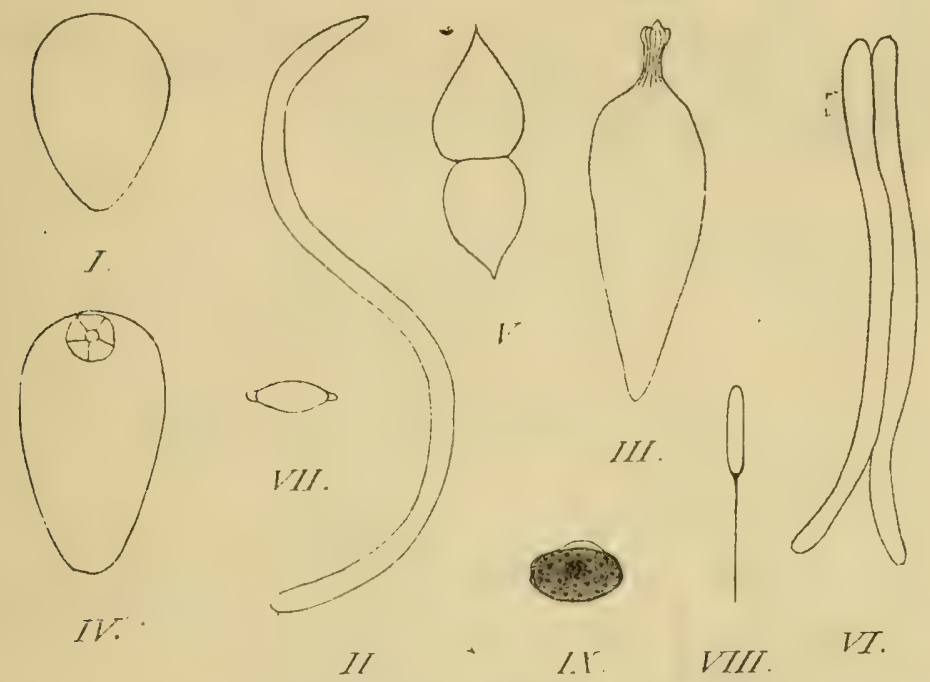

1'xu. I. Monocystis Schéma. - VIG.II. Nenutocystis. Schéma.- Fig. III. Rhynchocystis. Schéma. - Fig. IV. Stomatophora Schéma. - Fig. V. Zygocystis Schéma. - Frg. VI, Pleurocystis Schéma. - FIG. VII. Sporocystes des genres Monocystis, Nematocystis, Rhynchocystis, Stomatophora, Zygocystis et Pleurocystis. - FIG. VIII. Sporocystes de Urospora tubificis, d'après R. Lankester.-FIG. IX. Sporocyste (Amphiont) de Schaudinnella, d'après Nusbaum.

G. Stomatophora Drzewecki. Corps ovoïde ou sphérique. Pôle antérieur muni d'une ventouse pétaloïde à côtes radiées. Sporocystes biconiques à pôles semblables non appendiculés (fig. Iv).

G. Zygocystis Stein. Individus tonjours en association ì l'état adulte. Accolement par le pôle antérieur. Corps piriforme ou sphérique. Sporocystes biconiques à pôles semblables (fig. v).

G. Pleurocy stis g. nov. Trophozoïtes toujour's associés. Accolement longitudinal, latéral. Sporocystes biconiques à pôles semblables (fig. vI). 
G. Urospora A. Schneider. Sporocystes ovalaires plus ou moins allongés, munis d'un filament caudal (fig. virr.)

G. Schaudinnella Nusbaum. Individus mâles et individus femelles distincts dès le jeune âge. Association temporaire durant la vie végétative d'individus de même sexe ou de sexe différent. Sporocystes (Amphiontes) arrondis, mobiles dans le jeune âge. Sporozoïtes arrondis (fig. Ix).

Le tableau suivant résume les caractères distinctifs de ces genres, il permet de classer immédiatement une Grégarine quelconque rencontrée dans les Oligochètes et pourra ainsi rendre quelques services aux chercheurs.

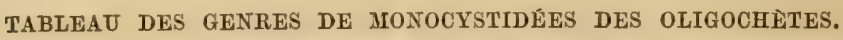

Sporocystes sphériques, mobiles à l'état jeune. Sporozoïtes sphériques. SCHAUDINNELIA Nus-

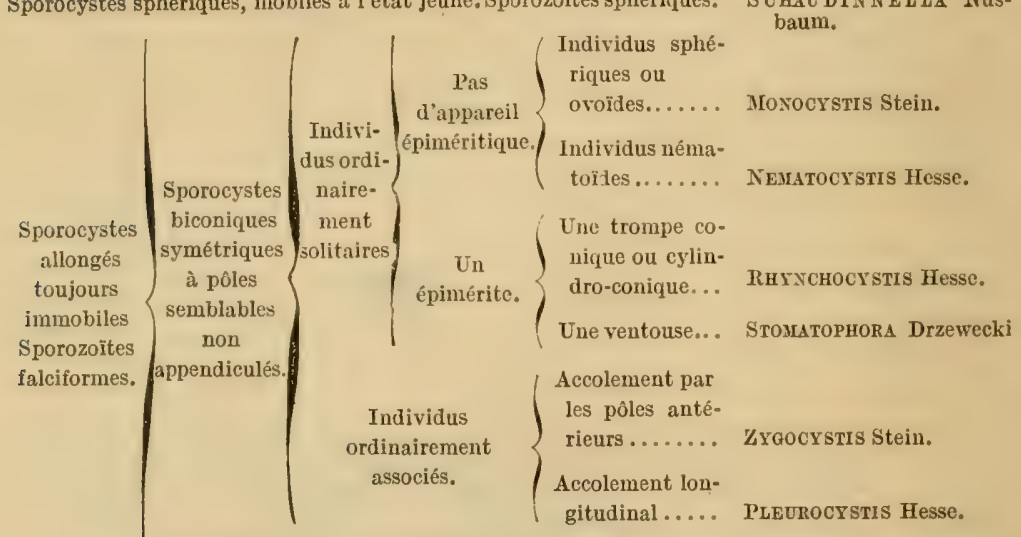

Sporocystes ovalaires avec filament caudal........ UrosporA A. Schneider.

\section{Habitat.}

Nous avons vu déjà que presque toutes les espèces étudiées dans ce mémoire sont des parasites cœlomiques, une seule est parasite de l'intestin, il s'agit très probablement de Monocystis mitis Leidy.

Parmi les Monocystidées cœlomiques, les unes se rencontrent uniquement dans la cavité générale, elles sont peu nom- 
breuses, nous en avons observé seulement deux : $M$. Bretscheri n. sp. et M. Michaelseni n. sp.

D'autres peuvent habiter aussi bien la cavité générale que les vésicules séminales, telles sont : $\boldsymbol{M}$. herculea Bosanquet, Nematocystis vermicularis n. sp., M. Le Mêmei n. sp., M. Elmassiani, n. sp., M. Duboscqi n. sp.

Il en est que l'on trouve localisées dans les vésicules séminales comme : $M$. lumbrici Henle, $M$. agilis Stein, $R$. pilosa Cuénot, $R$. porrecta Schmidt, M. hirsuta n. sp., M. turbo n. sp., M. lumbrici olidi Schmidt, $M$. villosa n. sp., $M$. crenulata n. sp., M. Cognettii n. sp., N. lumbricoüdes n. sp., N. anguillula n.sp., Stomatophora coronata Hesse, S. diadema n. sp., Zygocystis cometa Stein, Z. Legeri n. sp., Z. pilosa n. sp.

Quelques-unes s'observent dans les vésicules séminales et sur le bord libre des pavillons séminaux : $M$. striata $\mathrm{n}$. sp. et $R$. pilosa (variété $\mathrm{C}$ ).

D'autres enfin sont localisées dans l'intérieur des pavillons ciliés, ce sont $M$. magna Schmidt et Pleurocystis Cuenoti n. sp.

\section{Monocystidées intestinales.}

Nous n'avons rencontré qu'une seule espèce dans l'intestin des Oligochètes que nous avons étudiés et nous n'en connaissons que les états végétatifs; nous la croyons très voisine de Monocystis mitis Leidy.

\section{Monocystis mitis Leidy.}

LeIDy (1882) a trouvé dans l'intestin de Distichopus silvestris Leidy, une petite Grégarine fusiforme qu'il a nommé Monocystis mitis. Il considère cette espèce comme voisine de $M$. enchytraei Kölliker.

Nous avons observé un parasite très voisin sinon identique dans l'intestin de Fridericia polycheta Bretscher recueillis en grand nombre à Montessaux (Haute-Saône). Comme nous n'avons pas observé les sporocystes, il nous paraît inutile pour 
l'instant de séparer les deux espèces bien qu'elles habitent des hôtes différents.

Diagnose. - Monocystidée fusiforme mesurant 180 \% de long sur 50 u. d'épaisseur. Extrémité antérieure pourvue d'un. mucron conique ou renflée en boule; extrémité postérieure pointue; Epicyte à stries longitudinales parfois assez accentuées. Myocyte présentant des fibrilles longitudinales très fines. Endoplasme. dense, finement granuleux. Noyau arrondi ou prismatique, renfermant un karyosome sphérique et des grains chromatiques dispersés sur le réseau de linine.

Kystes et sporocystes non observés (Leidy a considéré comme spores de la Grégarine des parasites de celle-ci appartenant au genre Metchnikovella Caullery et Mesnil, et que nous étudierons plus loin).

Навітат : Intestin de Fridericia polycheta Bretsch.

Monocystis mitis n'est pas très commune, mais elle est toujours très abondante dans un hôte envahi. L'épimérite piqué dans les cellules épithéliales de l'intestin

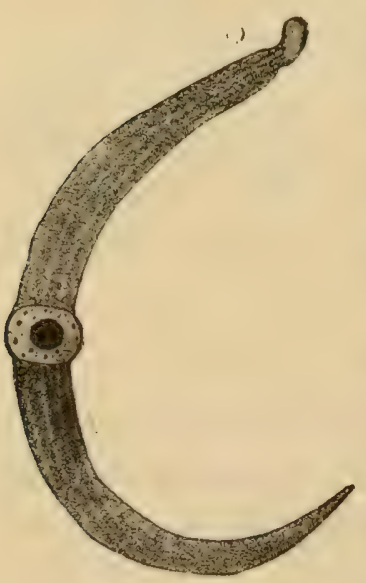

FIG. X. Monocystis milis Leidy. Parasite courbé en are; bandes longitudinales de sarcocyte; épimérite en bouton ; noyau bosselant la surface du corps, $] \times 750$. maintient le parasite dont le corps flotte librement dans la cavité intestinale. Souvent les Grégarines sont accumulées en certains points en telle quantité qu'elles obstruent presque complètement la lumière intestinale.

Le parasite a la forme d'un fuseau parfois court et assez renflé vers son milieu, parfois au contraire très allongé et mince. Les individus courts sont en général rectilignes, quelquefois cependant ils sont un peu courbés en arc. Les formes allongées présentent toujours cette incurvation (fig. $\mathrm{x}$ ), en outre leur extrémité postérieure s'infléchit souvent légèrement en crosse. 
Les formes courtes mesurent jusqu'à $40 \mu$ d'épaisseur pour $160 \%$ de long, quant aux individus allongés leur grand axe atteint quelquefois 240 \% de long, l'épaisseur du corps ne dépassant pas $15 \mu$.

Les mouvements du parasite sont excessivement lents, souvent même il paraît complètement immobile. On n'observe pas ici comme chez beaucoup d'autres Monocystis de course des granulations cytoplasmiques d'une extrémité du corps à l'autre, il existe seulement une sorte de mouvement pendulaire de l'extrémité postérieure, l'extrémité antérieure restant immobile (fig. XI, a); lorsque la Grégarine est détachée de l'épithélium, l'extrémité antérieure peut présenter ce même mouvement pendant que l'extrémité opposée se tient immobile (fig. XI, b).

L'extrémité antérieure est légèrement renflée tandis que l'extrémité postérieure est acuminée. Au pôle antérieur s'observe une sorte d'appareil épiméritique rappelant tout à fait celui de Schaudinnella henlae Nusbaum et voisin par

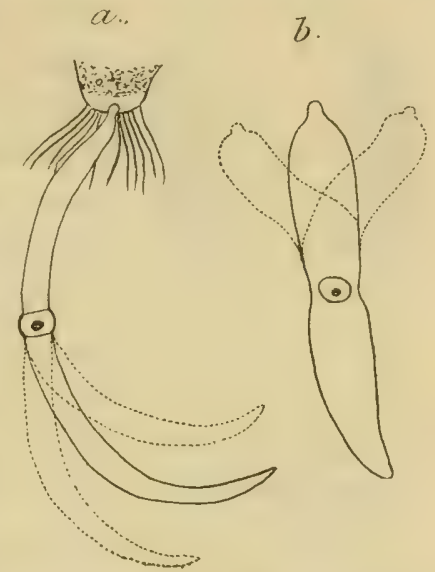

FIc. XI. Monocystis mitis Leidy. Schéma montrant le mouvement de l'extrémité postérieure d'un individu fixé ì une cellule intestinale, et le mouvement de l'extrémité antérieure d'un individu libre $\times 500$ conséquent de celui de Mon. ascidiae. C'est un cône de cytoplasme hyalin qui s'enfonce dans la cellule épithéliale de l'hôte. Il n'est pas recouvert par l'ectoplasme, les stries de l'épicyte s'arrêtent à sa base. Parfois, il se renfle en un bouton et se courbe légèrement constituant une sorte de genou. Les colorants plasmatiques, Eosine et Vert-lumière en particulier, le teintent avec plus d'intensité que le cytoplasme, il montre alors une consistance homogène et est complètement dépourvu de granulations. Mais cette structure varie, il arrive souvent que l'axe seul de cet épimérite présente ainsi une consistance. 
homogène et possède une grande affinité pour les colorants acides, les bords sont alors constitués par un cytoplasme granuleux; on observe aussi une disposition inverse, l'axe étant formé de cytoplasme granuleux et la couche périphérique de cytoplasme dense, homogène, fortement colorable. Ce changement de structure s'observe également in-vivo, le centre du mucron paraît alors quelquefois occupé par une vacuole plus ou moins développée.

La surface du corps est ornée de crêtes assez larges, parfois

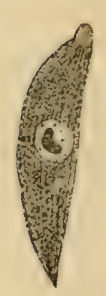

'XIT.

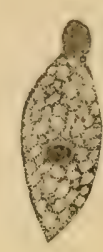

XIII.

Fr. XII. Jeune M. Mitis $\times 1200 .-$ FIG. XIII. Monocystis mitis à noyau en dégénérescence. Structure alvéolaire du cyto. plasme $\times 1200$. très élevées et qui se colorent intensivement par le Fer. Ce ne sont pas là de simples ornements épicytaires, le sarcocyte contribue à leur formation. Elles sont séparées par des sillons d'une largeur moindre que la leur. Ces côtes vont en s'amincissant vers les deux extrémités; elles sont disposées suivant des lignes méridiennes régulières et divergent en avant à partir de la base du mucron épiméritique sur lequel elles ne s'avancent pas. L'ébauche de ces côtes apparaît chez les jeunes individus sous forme de minces stries épicytaires assez espacées et partant de la base d'un mucron hyaloplasmique en bouton arrondi (fig. XII).

La couche de sarcocyte est mince et de consistance homogène ; c'est elle qui constitue les côtes ectoplasmiques en se disposant en bandes longitudinales plus ou moins épaisses, séparées par des sillons de largeur variable.

Les fibrilles du myocyte sont fines et serrées, elles dessinent des lignes méridiennes longitudinales régulièrement espacées; difficiles à voir dans les préparations colorées, elles sont toujours facilement perceptibles sur le parasite vivant, en mouvement. Je n'ai pas observé de fibrilles transversales, leur absence expliquerait le manque de mouvement dans les granulations cytoplasmiques, mouvement qui existe chez presque toutes les autres Monocystidées des Oligochètes, 
L'endoplasme finement granuleux est très dense, les plus forts grossissements dont j'ai pu disposer ne m'ont pas permis d'y déceler une structure alvéolaire, sauf cependant chez quelques individus parasités, ou dans des formes massives et très petites (fig. XIII) à noyau paraissant en dégénérescence. Les vacuoles de taille inégale renferment parfois des grains de paramylon.

Le noyau est logé au voisinage du milieu du corps ; en général ovoïde ou sphérique, il a quelquefois une forme cubique. Cet aspect s'observe même in-vivo, il ne peut, par suite, pas être attribué à l'action des fixateurs. Lorsqu'il est ovoïde, son grand axe est placé perpendiculairement à celui de la Grégarine; souvent dans ce cas, le corps du parasite présente une constriction au niveau du noyau (fig. xI, b). Quadrangulaire, il est plus volumineux et distend parfois le corps de la Grégarine qui montre à son niveau un renflement annulaire formant une sorte de ceinture (fig. $x$ et Xr a).

La membrane nucléaire se colore avec intensité par les colorants de la chromatine. Le noyau renferme un gros karyosome sphérique et des grains chromatiques peu volumineux dispersés sur le fin réseau de linine. Le karyosome paraît massif ; on n'observe que rarement dans son intérieur les vacuoles que nous allons rencontrer chez la plupart des autres Monocystis des Oligochètes.

Je n'ai observé ni accouplement, ni kyste, ni sporocystes.

Très fréquemment, le corps est rempli de corpuscules naviculaires que Leidy considérait comme des spores de la Grégarine, ce sont les spores d'un parasite appartenant au genre Metchnikovella Caullery et Mesnil et dont nous dirons quelques mots dans un chapitre ultérieur.

\section{Monocystidées de la cavité générale.}

Etudions d'abord les deux seules espèces que nous ayions rencontrées uniquement dans la cavité générale, 


\section{Monocystis Bretscherı n. sp.}

Des individus de Fridericia polycheta Bretscher recueillis à Montessaux (Haute-Sâone), m'ont montré assez fréquemment une Grégarine cœlomique volumineuse d'un blanc mat, facilement visible à travers les téguments de l'hôte et que

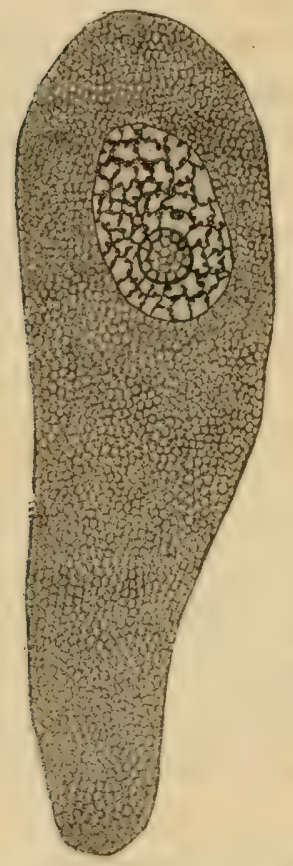

FIG. XIV, M. Bretscheri. Forme libre du cœlome $\times 1200$. j'appellerai $M$. Bretscheri, la dédiant au savant spécialiste suisse qui a bien voulu me déterminer les hôtes.

C'est une Monocystidée ellipsoïdale très grande, mesurant jusqu'à $200 \mu$. de long sur $50 \mu$ d'épaisseur. Elle présente un ectoplasme mince sur lequel je n'ai pas observé d'ornementations épicytaires.

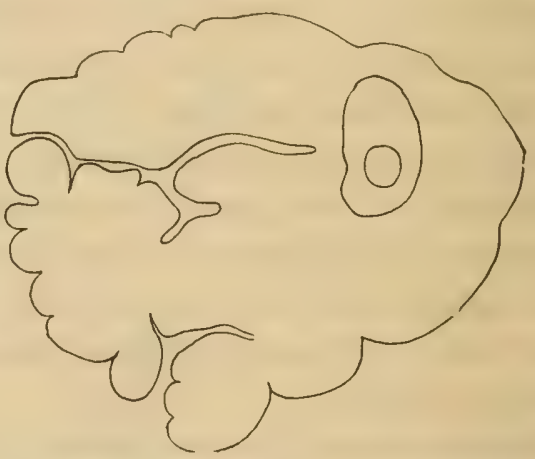

Fig. XV. M. Bretscheri. Forme du parasite englobé dans les cellules chloragogènes $\times 500$.

L'endoplasme finement granuleux est dense, creusé de très petites alvéoles régulières renfermant des grains de paramylon sphériques, de taille remarquablement uniforme. On y voit souvent des masses chromatoïdes de volume et de forme variables.

Il y a un gros noyau sphérique qui renferme un karyosome central à moelle vacuolaire et dont la couche externe seule se teint par le carmin; et des grains de chromatine abondants 
disposés sur un réseau de linine à épais filaments (fig. xrv).

La plupart des individus sont libres dans le colome; mais un certain nombre sont englobés dans la couche de cellules chloragogènes qui entourent l'intestin et ils présentent alors des transformations curieuses que nous retrouverons chez les Monocystidées génitales attaquées par les phagocytes. Ils sont hypertrophiés et peuvent atteindre jusqu'à 180 ".. de diamètre, ils sont en outre découpés en lobes profonds plus ou moins réguliers qui leur donnent un aspect très particulier (fig. $\mathrm{xv}$ ).

Les kystes sont sphériques et renferment des sporocystes de $18 \mu$ sur $8 \mu$ ayant la forme et la structure si souvent décrites pour les spores des Monocystis du Lombric et renfermant 8 sporozoïtes.

\section{Monocystis Mıchaelsem n. sp.}

On observe souvent dans le cœlome des Pheretima hawayana Rosa recueillis dans les serres du Jardin des Plantes de Grenoble, une quantité considérable de petites masses d'un blanc laiteux, visibles comme $M$. Bretscheri à travers les téguments de l'hôte; ce sont également des Grégarines monocystidées à divers stades de leur développement. J'ai dédié ce parasite à M. le professeur Michaelsen de Hambourg qui a eu l'amabilité de m'indiquer le nom des Oligochètes infestés.

Dragnose. - M. Michaelseni est une Monocystidée sphérique ou ovoïde, mesurant jusqu'à $225 \mu$ de diamètre ou $295 \%$ sur 230 p. Ses mouvements sont très lents. Il y a un ectoplasme peu'épais, mais à double contour très net. Endoplasme à granulations volumineuses donnant au parasite in-vivo un aspect très sombre, opaque. Noyau à un gros karyosome central.

Kystes ellipsoïdaux ayant de 235 à 300 p. de grand axe sur 170 à $220 \mu$ de petit axe.

Sporocystes naviculaires, fortement renflés à l'équateur, de 15 u. sur 9 .

Hоте : Pheretima hawayana Rosa 
Le parasite est particulièrement abondant dans le voisınage du clitellum de l'hôte, il forme là des amas très serrés. Rarement il est libre dans le cœlome, le plus souvent il se trouve entouré par une enveloppe de cellules conjonctives de l'hôte qui lui constituent une sorte de manteau et, se continuant en un pédicule creux le rattachent à la paroi du corps de l'hôte. Quelquefois il est inclus dans les tubes néphridiens.

Sa forme fondamentale est sphérique, mais elle peut se déformer de diverses manières, de sorte que l'on en voit d'ovoïdes ou d'ellipsoïdaux à grand axe très allongé. Il atteint de 200 à $300 \mu$ de diamètre. Ses mouvements très lents n'offrent rien de particulier.

Nous n'avons pas observé chez ce parasite de différenciation polaire qui permette de l'orienter.

L'ectoplasme comprend un épicyte sur la surface duquel je n'ai pas vu d'ornementation, et un sarcocyte de faible - épaisseur, hyalin. Je n'ai pas aperçu les fibrilles du myocyte.

L'endoplasme, qui paraît très sombre in-vivo, est constitué par un cytoplasme granuleux, alvéolaire, dont les mailles sont occupées par des grains de réserve volumineux.

Sur les coupes colorées par la méthode de Benda (SafranineVert lumière, ou Rouge Magenta-Vert lumière), après fixation par le Flemming, les parasites ne présentent pas tous la même coloration; certains d'entre eux ont une teinte très foncée, leur cytoplasme se colore en rouge brun; d'autres, à teinte beaucoup plus claire ont un cytoplasme rouge pâle avec quelques tâches bleuâtres. Il ne s'agit pas là d'une différence sexuelle car on observe que, dans un kyste, les deux individus conjugués présentent la même teinte. Il existe du reste des kystes à coloration foncée et des kystes à teinte claire et la même différence s'observe jusque dans les sporocystes. Faut-il voir là une véritable différence spécifique? Je n'ose me prononcer pour l'affirmative, car la colorabilité seule est variable, la structure cytoplasmique et nucléaire est absolument la même chez les formes claires et chez les formes foncées. 
Le noyau sphérique présente une membrane chromatique et renferme un gros karyosome vacuolaire central. sphérique également. Le reste du noyau est occupé par le réticulum lininien dont les mailles sont remplies de fines granulations achromatiques. Le réseau supporte des grains chromatiques assez nombreux petits et irréguliers vers le centre, plus volumineux et sphériques au voisinage de la membrane (fig. xvI).

J'ai observé chez cette espèce des enkystements solitaires qui sont toujours un

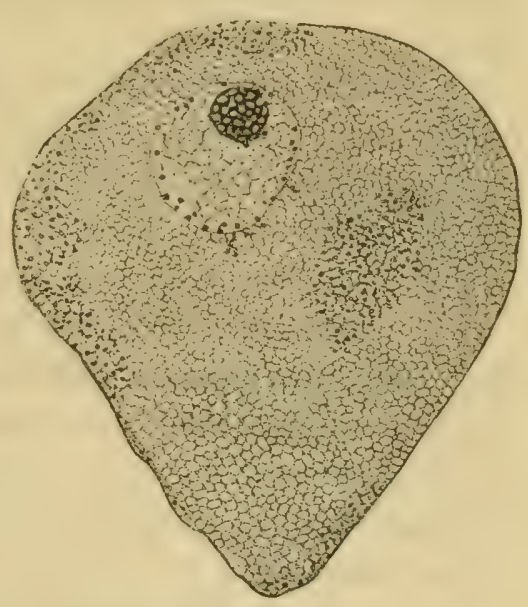

Fra. XVI. M. Michaelseni $\times 500$. prélude de dégénérescence.

Les kystes à deux individus sont ellipsoïdaux et mesurent 23. à 300 \% de long sur 170 à 220 ;. d'épaisseur. Il existe aussi

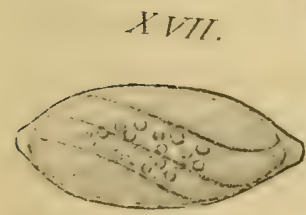

$X / X$

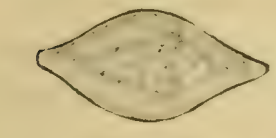

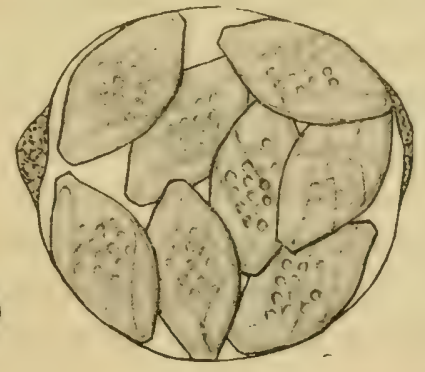

XTIII.
FIG. XVII., Sporocyste de MI. Michaelseni x 1.300.-FIG. XVIII, Kyste de $\boldsymbol{M}$. maerospora $\times$ 500. - FIG. XIX. Sporocysto de $\boldsymbol{M}$. macrospora $\times 1300$. des kystes à 3 individus dont je ne connais pas le sort ultérieur.

Les sporocystes naviculaires (fig. xvII) ont la structure de ceux des Monocystis de Lumbricus herculeus; mais ils sont moins allongés, plus fortement renflés à l'équateur et mesurent $15 \mu$ sur $9 \mu$. Ils renferment 8 sporozoïtes à disposition normale. 
Je ne connais pas le développement du parasite.

A côté des kystes de $M$. Michaelseni, on trouve dans les mêmes amas d'autres kystes dont je n'ai pu déterminer l'origine.

Ces kystes (fig. xvIII) ont une paroi mince présentant parfois un ou plusieurs renflements, et ils contiennent 4, 6,8 ou 16 sporocystes très volumineux, ellipsoïdaux, montrant à chaque extrémité un petit bouton aplati et mesurant 20 à $24 \mu$ de long sur 12 à $13 \mu$ de large (fig. XIX).

Ces sporocystes se rencontrent souvent isolément; on en observe parfois dans les vésicules séminales. Ils existent également chez $P h$. rodericensis Grube, où ne se rencontre jamais M. Michaelseni.

L'état végétatif du parasite qui donne ces sporocystes nous est inconnu.

Nous le désignerons (à cause de ses volumineux sporocystes) sous le nom de $M$. macrospora.

\section{Monocystidées génitales.}

Nous avons vu que l'on pouvait observer chez les Monocystidées génitales une certaine spécialisation dans l'habitat, et énuméré les divers groupes que l'on pouvait distinguer à ce point de vue; nous n'étudierons pas les espèces dans l'ordre de ce groupement, mais, pour faciliter les comparaisons entre diverses espèces nous décrirons préférablement à la suite les unes des autres celles qui appartiennent à un même genre, et dans le même genre celles qui parasitent le même hôte ; par suite, les tableaux placés en tête du mémoire indiqueront l'ordre de cette étude.

\section{Monocystis Iumbrici Henle.}

Historique ét synonyme. - Monocystis lumbrici Henle est l'espèce la plus anciennement connue des Monocystis du Lombric. Elle a été vue pour la première fois par DUJARDIN (1835) dans le cœlome du Ver de terre et considérée comme un 
Flagellé, le Proteus tenax O.-F. Müller. Surtray (1836) frappé par ses mouvements caractéristiques l'appelle " Sablier protéiforme "; enfin Henle (1845) décrit pour la première fois le bouquet de poils terminal et donne au parasite le nom de Gregarina lumbrici. STEIN (1848) paraît avoir revu cette espèce ; les figures 1 et 2 , planche IX de cet auteur représentent en effet très probablement $G$. lumbrici Henle, quoique les poils ne soient pas représentés, mais les figures 3 et 4 planche IX se rapportent certainement à un autre parasite que Stein confond avec le précédent sous le nom de Monocystis agilis.

Schmidt (1854) retrouve le même organisme et lui donne encore un nom nouveau, Monocystis cristata, il décrit avec une grande exactitude les mouvements en sablier du cytoplasme déjà remarqués par les observateurs précédents; les poils terminaux ne sont pas pour lui filiformes, mais ils se présentent comme des expansions arrondies.

Ruschhaupt (1885) désigne le parasite sous le nom de Monocystis cristata Schmidt, il en donne une très bonne figure et déclare avoir vu les poils se renfler parfois en boule et prendre l'aspect décrit par Schmidt.

Enfin Cuénot (1901) écrit une diagnose précise de cette espèce et en débrouille la synonymie, cependant il n'a pas remarqué que, parmi les Grégarines appelées $M$. agilis par Stein, il y a manifestement plusieurs espèces.

Habitat et SiÈge. - J'ai rencontré assez communément M. Iumbrici dans les vésicules séminales de divers Lombriciens : Lumbricus terrestris L., Müll., L. rubellus Hoffm., L. castaneus Sav. provenant de diverses localités de la Franche-Comté, du Dauphiné ou de la Provence. C'est chez L. rubellus qu'elle m'a paru le plus abondante. Je n'ai jamais observé ce parasite dans la cavité générale où cependant il a été signalé par diver's observateurs. Tous les individus que j'ai rencontrés étaient libres dans le fluide séminal, jamais je n'ai vu de stades, même très jeunes dans les blastophores de l'hôte; $M$. lumbrici serait donc extra-cellulaire pendant toute la durée de son développement. 
Forme, imensions et mouvements. La forme du parasite est variable. Jeune et au repos, il est ovoïde puis il devient un ellipsoïde étroit et allongé et conserve cet aspect jusqu'à la fin de son développement chez L. rubellus; chez les autres hôtes, le petit axe, s'accroissant proportionnellement plus vite que le grand axe la Grégarine prend la forme d'un ovoïde ou d'un ellipsoïde fortement renflé.

Les dimensions varient aussi dans de grandes limites avec les hôtes ; chez L. casta-

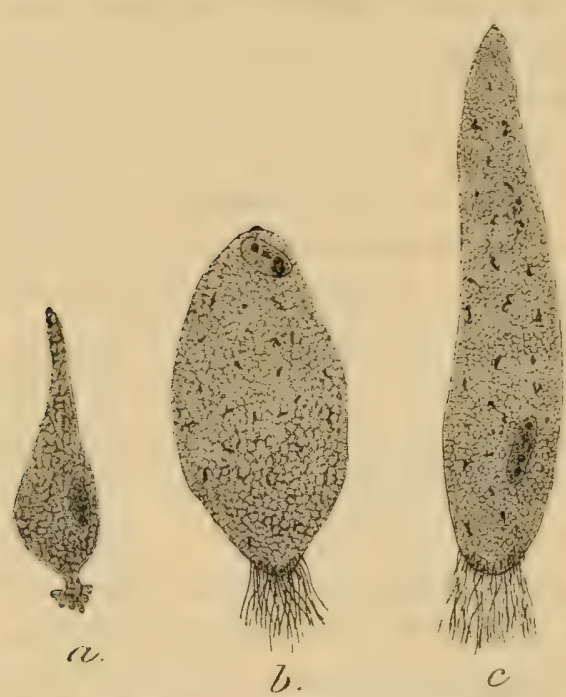

FIG. XX. M. lumbrici $\times 250 . a$, forme parasite de $L$.castaneus; $b$, forme parasite de $L$. terrestris; $c$, forme parasite de $L$. rubellus. neus, le parasite n'atteint pas souvent $200 \mu$ sur $50 \mu$; chez $L$. terrestris il est un peu plus volumineux, $200 \mu$ sur $60 \%$ en moyenne, enfin chez $L$. rubellus ses dimensions ordinaires à l'état adulte sont de 350 à $400 \mu$, sur 70 à $80 \mu$. La figure $\mathrm{xx}$ représente côte à côte ces trois formes qui diffèrent assez notablement.

La forme générale du corps est légèrement modifiée par la présence à l'un des pôles d'un petit mucron conique et à l'autre d'une touffe de poils d'aspect variable, je reparlerai plus loin de ces formations en décrivant l'ectoplasme du parasite. Une autre cause modifie beaucoup plus profondément la forme de $M$. lumbrici, c'est le mouvement continuel qui agite son cytoplasme et qui est produit par la contractibilité de ses fibrilles myocystiques. Tous les observateurs ont remarqué la course rapide des granulations cytoplasmiques, des grains de paramylon et du noyau lui-même qui sont chassés sans cesse d'une extrémité du corps à l'autre. 
C'est ce mouvement si caractéristique qui avait valu au parasite le nom de "Sablier protéiforme " donné par Suriray (1836); il a été très bien décrit par Sснмidt (1854) et il est inutile de revenir ici sur cette description. Rappelons seulement que ce mouvement est très rapide et qu'il se produit toujours en ligne droite, suivant la direction du grand axe de la Grégarine ; ces faits permettent de distinguer au premier coup d'œil, dans les préparations in-vivo, M. lumbrici et une autre espèce décrite plus loin $M$. agilis où le mouvement plus lent ne se fait pas en ligne droite.

Par suite de ces mouvements, le corps du parasite peut prendre l'aspect d'un fuseau, d'un sablier, d'une toupie, d'une massue à manche étroit et strié longitudinalement et portant à son extrémité la touffe de poils caractéristique. Nous retrouverons ces mêmes variations de forme chez les autres espèces à mouvements également rapides et rectilignes, $M$. turbo et St. coronata par exemple.

Comment convient-il d'orienter le corps du parasite? Celuici n'étant jamais fixé, il ne présente pas à son pôle antérieur d'appareil fixateur différencié comme il en a été signalé chez quelques Monocystidées ou comme ceux que nous décrivons plus loin chez d'autres espèces parasites des Lombrics. Mais Henle (1845) avait déjà observé que l'une des extrémités porte un bouquet de cils fins, immobiles. Schмпd (1854) a revu la même formation, non plus sous forme de poils fins, mais comme un faisceau de filaments courts, un peu allongés, arrondis au sommet (" einen Bündel kurzer, länglicher, oben abgerundeter Fortsätze »).

Pour Ruschinupt (1885) l'aspect des filaments est variable, ils peuvent se raccourcir, se renfler en boules puis redevenir filiformes; Ruschнаupt considère l'extrémité qui les porte comme antérieure, il croit que les filaments peuvent se coller aux cellules amœboïdes des vésicules séminales.

Cú́not (1901) représente les poils comme des filaments longs et fins; de même que Ruschhaupt il regarde le bouquet de $\triangle R O B, D E$ ZOOL, EXP. ET GEN。 - 5e SERIE. - T. III. - (II)。 
poils comme antérieur. BRASIL (1905 b) n'a pas vu ce bouquet de poils, cela tient sans doute à ce que cet observateur a eu affaire non à $M$. lumbrici, mais à $M$. agilis qui sera décrite plus loin.

Je n'ai pas remarqué in-vivo les changements de forme constatés par RuschHAUPT, mais dans les préparations fixées et colorées, le bouquet de poils peut se présenter sous trois aspects différents. Ces poils peuvent être courts et larges, ce sont plutôt de petites émergences cylindro-coniques que des filaments, parfois même ils sont renflés en boule à leur extrémité (fig. 2) ; c'est l'aspect décrit et figuré par Schмid (1854, fig. 13, 14 pl. XIV), on l'observe surtout dans les stades jeunes; chez les parasites plus âgés on a, ou bien un bouquet de poils longs et fins (fig. 7), un peu plus épais à la base qu'au sommet, tels que le représentent RuschHAupt et CuÉNOT, ou bien une sorte de feutrage (fig. 3) constitué par des poils tassés et enchevêtrés, entre lesquels semble s'étendre une couche cytoplasmique granuleuse. Comme l'a très bien vu RuschHaupt, ces filaments ne sont pas tout à fait terminaux, ils s'insèrent à une faible distance de l'extrémité, suivant une ligne circulaire plus ou moins régulière. Nous considérons ces poils comme homologues aux expansions postérieures de Zygocystis cometa Stein. LÉGER (1892) pense qu'ils ne sont pas autre chose que des prolongements des côtes de l'épicyte ; c'est aussi notre avis ; en décrivant l'épicyte, nous montrerons comment il faut interpréter la variabilité d'aspect qu'ils peuvent présenter.

Ainsi, les poils de $M$. lumbrici seraient placés à l'extrémité postérieure du corps et non à l'extrémité antérieure. Si l'on examine celle-ci avec attention, on y observe une toute petite masse de cytoplasme dense, non granuleux, où ne pénètrent jamais les inclusions endoplasmiques et qui fait saillie sous forme d'une éminence conique, peu développée, non recouverte par le sarcocyte et sur laquelle l'épicyte lui-même est excessivement mince et sans ornementation (fig. 1) ; c'est en effet à partir de la base de ce mucron rudimentaire que rayonnent 
les stries épicytaires. Une formation semblable existe chez d'autres espèces, mais elle est plus développée et sert même parfois à fixer le parasite à la cellule hôte; cette formation a donc les caractères d'un véritable épimérite et on doit la considérer comme antérieure, le petit mucron de $M$. lumbrici qui peut lui être homologué à juste raison doit donc être lui aussi regardé comme appartenant au pôle antérieur. Il a été nettement dessiné par Ruschнaupt (1885, fig. 1, taf xxII), mais cet observateur n'en a pas étudié la structure et il le considère comme une pointe postérieure.

Le pôle postérieur est arrondi et non mucroné; parfois, cependant, la région située en arrière de l'insertion des poils se présente comme une petite bosse arrondie faisant fortement saillie; mais on ne peut la confondre avec le mucron antérieur parce qu'elle est toujours recouverte par l'ectoplasme.

Ectoplasme. - L'ectoplasme est formé par les trois couches ordinaires : épicyte, sarcocyte et myocyte; nous n'avons pas observé ici la "Gallertschichte " de Schewrakoff (1894).

L'épicyte est mince; son épaisseur paraît uniforme sur toute la surface du corps, sauf sur le mucron antérieur où il est très réduit, peut-être même absent.

La couche de sarcocyte est, elle aussi, assez mince et d'une épaisseur sensiblement uniforme, seulement interrompue sur le mucron antérieur. Elle est creusée de sillons méridiens qui séparent des côtes parallèles; ces côtes sont peu saillantes, à peine perceptibles chez le parasite au repos, mais lorsque celuici se contracte et prend par exemple la forme d'une toupie ou d'une massue, les côtes ectoplasmiques diminuent de largeur et deviennent au contraire très épaisses dans la région contractée qui prend ainsi un aspect cannelé caractéristique; une coupe. transversale du parasite a alors tout à fait l'aspect d'une collerette plus ou moins régulière (fig. $\mathrm{xxI}$ ).

Ce sont ces côtes qui se prolongent postérieurement pour constituer le bouquet de poils postérieurs. Lorsque la côte se prolonge peu, on a l'aspect représenté par la figure 3 qui rap-. 
pelle beaucoup les figures 13 et 14, planche XIV de Schmid (1854) ; elle peut au contraire s'allonger beaucoup et se diviser en plusieurs filaments, réduits presque à la seule cuticule (fig. 4); ces filaments, très fins et très nombreux peuvent se tasser, s'enchevêtrer les uns dans les autres et, s'ils renferment un peu de sarcocyte, il se produit entre

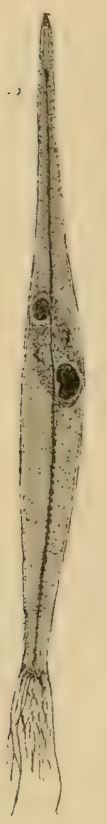

FIG.XXII. M.lumbrici, présentant un profond sillon médian $\times 600$. eux des fusions partielles qui donnent naissance au feutrage dont nous avons parlé plus haut (fig. 3). J'ai observé aussi une Grégarine chez laquelle un sillon profond (fig. XXII) séparait le corps en 2 parties sensiblement symétriques, de sorte que le parasite avait l'aspect des Cystobia chirodotae figurées par DoGIEL (1906).

Les fibrilles du myocyte sont parallèles et dessinent des stries circulaires sur le corps de la Grégarine; elles paraissent formées comme l'a vu Van Beneden (1872) chez Porospora du Homard, d'une série de petites granulations très rapprochées les unes des autres et ordon nées en rangées inégalement espacées, disposées par groupes de deux très rapprochées et séparées du groupe voisin par un espace un peu plus considérable (fig. 8). Le Fer les colore très fortement.

Il existe en outre des fibrilles longitudinales (fig. xxuI) parallèles aux sillons ectoplasmiques et qui apparaissent dans les frottis comme des lignes très fines, régulièrement espacées. De telles fibrilles ont déjà été signalées chez d'autres espèces de Grégarines par Bütschli (1882, fig. 4, taf xxxiv) par Claparéde (1861) et par LIeberkühn (1865). Caullery et Mesnil (1899) ont décrit aussi des myonèmes longitudinaux chez les Selenidium.

L'entocyte des individus adultes est constitué par un cytoplasme très fluide et très mobile qui renferme deux sortes 
d'inclusions : des grains de paramylon et des granulations chromidiales.

Ce cytoplasme est creusé de grandes alvéoles polyédriques et de dimensions fort inégales. Les mailles du réseau d'abord remplies d'un hyaloplasme homogène, réfringent, sont ensuite occupées par les grains de réserve ; quant au réticulum spongioplasmique il comprend des travées assez étroites séparant les alvéoles et formées par une substance éosinophile dans laquelle s'observent des granulations de taille variable, faiblement colorables par l'Hématoxyline; à certains nœuds du réseau, ces travées se renflent de façon à constituer une masse volumineuse quelque-

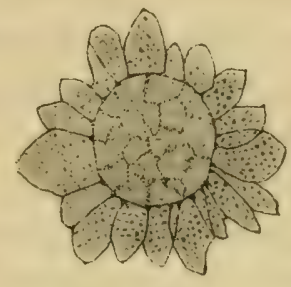

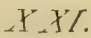

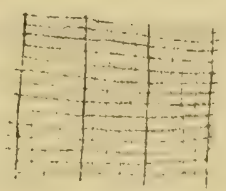

Y.XIII

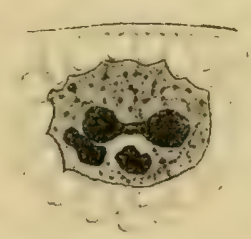

X.YIF

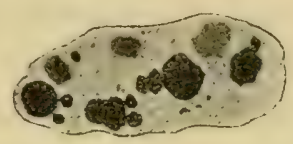

FYY

FIf. XXI. Coupe de la 'région postérieure contractée de $\boldsymbol{M}$. lum. brici $\times 1700 .-$ FIG. XXIII. Ectoplasme de $M$. lumbrici, montrant les sillons longitudinaux de la cuticule et les fibrilles plus fines du myocyte, les unes longitudinales les autres transversales $\times 1.300$. - FIG. XXIV. Noyau do M. lumbrici avec trois karyosomes dont l'un est étiré en haltère $\times 1.300$. $-\mathbf{F}$ I. $\mathbf{X X V}$. Noyau de $\boldsymbol{M}$. lumbrici avec karyosome moniliforme et karyosomes bourgeonnant $\times 1.300$.

fois presque aussi grande que les alvéoles et dans laquelle il y a peu de granulations; elle se colore en gris dans les fortes imprégnations par le Fer; et au lieu d'être éosinophile, elle présente au contraire beaucoup d'affinités pour l'Orange. C'est dans ces nœuds orangophiles que s'accumulent les granulations chromidiales.

Celles-ci se colorent en bleu noirâtre par le Fer, et en rouge par le Mann, Il en existe deux sortes, les unes précèdent l'appa- 
rition des grains de paramylon, et elles disparaissent à mesure que se développent ces grains de réserve, servant peut-être dans une certaine mesure à leur édification. Ce sont des grains arrondis, assez volumineux, isolés ou rassemblés en séries moniliformes rectilignes ou circulaires; parfois on en voit un très gros suivi d'une série d'autres de plus en plus petits, de telle façon que l'ensemble prend la forme d'une alène; ces grains peuvent encore se souder en bâtonnets d'aspect varié ; le plus souvent, ils constituent des masses lancéolées paraissant renfermées dans des vacuoles (fig. 7).

D'autres sortes de masses chromatoïdes se rencontrent aussi dans le cytoplasme au moment de la disparition des grains de paramylon. Ce sont des sortes de gouttelettes divisées en deux zônes : la zone interne fortement colorée en noir par le Fer, la zone externe entourant la première, colorée en gris pâle (fig. 6). Si les granulations chromidiales de la première espèce jouent un rôle dans la synthèse des grains de réserve, il est probable que les gouttelettes représentent un stade de l'assimilation de ces réserves; nous discuterons cette question dans un chapitre ultérieur.

Les grains de paramylon ont des formes et un volume très variables, le plus souvent ce sont des masses ellipsoïdes de 6 . sur 3 i. en moyenne ; mais fréquemment, ces ellipsoïdes s'allongent en bâtonnets peu épais ou se renflent de façon à devenir des sphères assez volumineuses. En leur centre il existe une région fortement colorée en rouge par la Safranine et surtout par le Rouge Magenta et qui a la forme d'une sphère ou d'un court bâtonnet, il s'agit là sans doute d'une vacuole centrale comme le pensent Léger et Duboscq (1908) et non d'un grain d'excrétion comme le croit KusChaKewITSCH (1907).

Le noyau est un ellipsoïde allongé, assez régulier; il n'est sphérique que dans les jeunes stades; il se courbe en are dans certains cas et présente l'aspect figuré par Cú́not (1901, fig. 2, planche 18.) Sa position est variable car il est ballotté avec les grains de paramylon et transporté d'une extrémité 
du corps à l'autre par les contractions du parasite ; le plus souvent, il est incliné à $45^{\circ}$ sur le grand axe du corps, mais on l'observe aussi placé parallèlement ou perpendiculairement à cet axe.

La structure de ce noyau est variable, il contient toujours plusieurs karyosomes volumineux; en outre, il peut renfermer des grains de chromatine disposés sur un réseau ou en être totalement dépourvu, il est dans ce cas rempli par une substance granuleuse qui ne se teint pas par les colorants nucléaires. CuÉnot (1901) avait noté déjà la pluralité des karyosomes comme un caractère constant de cette espèce, et si BRASIL $(1905$ b) la nie, c'est qu'il a eu affaire à une autre espèce comme nous l'avons dit déjà. Le nombre des karyosomes est variable, j'en ai compté jusqu'à une douzaine de volumineux chez des individus de grande taille comme ceux que l'on rencontre communément chez L. rubellus (fig. 9.)

Ces karyosomes sont sphériques ou ovalaires le plus souvent, mais il en est aussi d'irréguliers; on peut en voir qui ont la forme d'haltères (fig. xxIv) ou de saucisses moniliformes (fig. $\mathbf{x x v}$ ); ce sont probablement des karyosomes en voie de fragmentation. Ainsi que l'a déjà constaté CuÉnot (1901), ces karyosomes sont creusés de vacuoles de dimensions variées et parfois assez nombreuses; cette structure vacuolaire est bien mise en évidence par le Mann, mais le Fer ne la décèle pas toujours. Cú́not déclare que les karyosomes "prennent les matières colorantes plus faiblement que la chromatine typique " nous verrons à propos du développement que ceci n'est exact que pour certains moments de l'évolution.

Cúnot (1901) et Brasil (1905 $b$ ) ont fait remarquer qu'il n'était guère possible de distinguer les kystes et les sporocystes des diverses espèces de Monocystis que l'on rencontre chez une même espèce de Lombric ; je n'ai guère été plus heureux que mes prédécesseurs à ce point de vue, aussi, faute de pouvoir attribuer à une espèce donnée des kystes et des sporocystes bien déterminés, je me contenterai de décrire ceux-ci d'une façon générale dans un chapitre particulier. 
Je ne m'attarderai pas non plus à décrire les processus de l'enkystement si bien étudiés par Hennegur (1888), Cúńnot (1901), puis par BRASIL (1905 b).

Pour tout ce qui concerne les phénomènes de la reproduction je renverrai done au travail de Brasil dont j'ai pu vérifier souvent l'exactitude.

Développement. - Les sporocystes mûrs s'ouvrent dans le tube digestif du Lombric, les sporozoïtes s'en échappent et gagnent les vésicules séminales; je n'ai pu les suivre durant cette migration qui doit s'opérer très rapidement si l'on en juge par les dimensions des plus jeunes stades observés dans les poches séminales; ces jeunes parasites ne présentent en effet pas plus de $16 \mu .5$ sur $10 \mu .5$, alors que les plus petits sporozoïtes ont une dizaine de $\mu_{\text {sur }} 1$ à $2 \mu$. Le sporozoïte ne s'allonge donc pas au début de sa croissance, il s'arrondit, ce n'est que plus tard que cette croissance devient plus rapide suivant une direction qui est celle du grand axe.

Le cytoplasme des formes jeunes est finement granuleux et très dense (fig. 5), il renferme de bonne heure des granulations chromidiales parfois nombreuses et volumineuses. Le noyau est sphérique ou ellipsoïdal, il contient d'abord un seul karyosome presque toujours excentrique, parfois même accolé à la membrane nucléaire et formant à l'un des pôles du noyau une espèce de calotte; il se colore en violet ou en rouge par le Mann ; le reste du noyau est occupé par de fines granulations bleues.

Bientôt on observe deux karyosomes placés aux deux extrémités d'un même diamètre, puis un plus grand nombre qui proviennent probablement de la fragmentation de l'unique karyosome primitif. Chez certains parasites, en effet, on voit le karyosome s'étirer en biscuit, dans d'autres, il a la forme d'un haltère, parfois il devient moniliforme comme nous l'avons $\mathrm{vu}$ assez souvent dans des cas pathologiques; toutes ces formes représentent sans doute des stades de fragmentation qui conduisent à la pluralité des karyosomes, 
En général les karyosomes sont vacuolaires, mais ils peuvent aussi être massifs; dans ce cas, leur coloration est aussi intense que celle de la chromatine typique. Non seulement, les karyosomes se multiplient par bipartition, mais il semble en outre qu'ils peuvent donner naissance ì des granulations chromatiques de diverses grosseurs par un véritable processus de bourgeonnement. BERNDT (1902) a décrit une modalité particulière de ce processus chez les Grégarines du Tenebrio molitor, modalité retrouvée par KuschaKewitsch (1907); nous n'avons rien vu de semblable chez $M$. lumbrici; les figures que nous avons eu sous les yeux se rapprochent davantage de celles que représentent LÉGER et DUBOSCQ (1904) chez Stylorhynchus, et Brasil $(1905 b)$ chez des individus accouplés de Monocystis des Lombrics. LÉGER et DuboscQ ont remarqué chez Stylorhynchus que les grains bourgeonnés sont basophiles ou acidophiles suivant leur taille; chez M. lumbrici, tous ces grains sont colorés en violet par le Mann, ils seraient donc tous basophiles. A mesure qu'augmente le nombre de ces granulations, le nombre des vacuoles s'accroît dans les karyosomes et la colorabilité de ces karyosomes diminue. Les grains chromatiques ainsi émis dans le noyau restent d'abord disposés en cercle autour du karyosome qui leur a donné naissance, parfois accolés à lui, parfois rattachés simplement par un mince filament. Ils se dispersent ensuite dans le suc nucléaire et se disposent sur le réseau de linine très délicat et souvent difficile à voir. Il est surtout bien mis en évidence par le Mann qui le colore en bleu pâle. Je n'ai pu établir par quel processus les karyosomes décolorés récupèrent la chromatine qu'ils ont émise dans le noyau, mais cette récupération se produit très certainement, car les parasites prêts à l'accouplement sont pourvus de karyosomes bien colorés en même temps que d'un réseau chromatique bien développé.

La membrane nucléaire est peu colorable, cependant, à certains moments, elle peut paraître très colorée par suite de l'accumulation à sa surface de fines granulations chromatiques 
très nombreuses. Cette membrane n'est pas toujours nette ; à certains moments le noyau prend une forme étoilée et la membrane disparaît vers les pointes de l'étoile mettant le suc nucléaire en rapport direct avec le cytoplasme (fig. 7) ; c'est là le " noyau flammé " de Wolters. Cuénot (1901) considère cet aspect particulier comme dû à un défaut de fixation, il n'en est rien et Drzewecki (1903) a pu l'observer in-vivo. Cette disparition de la membrane nucléaire facilite les échanges entre le noyau et le cytoplasme, peut-être même a-t-elle pour résultat de permettre le passage direct dans le cytoplasme d'une partie des granulations chromatiques du noyau. En tous cas si ce passage a lieu, la chromatine qui est ainsi émise hors du noyau subit une transformation car les granulations chromidiales du cytoplasme sont colorées par le Mann en rouge et non en violet comme la chromatine.

Ces formations chromidiales sont très caractéristiques de l'espèce ; sans doute, on en retrouve chez les autres Monocystis mais elles ont ici une forme particulière comme nous l'avons vu plus haut. Nous avons indiqué aussi le rôle qu'elles paraissent jouer dans l'évolution des grains de réserve. Lorsque ceux-ci sont bien développés les chromidies disparaissent, les nœuds du réseau cytoplasmique où elles étaient placées se colorent alors en violet pâle par le Fer et l'Hémalun, l'ensemble du cytoplasme se teint fortement par l'Orange, la Grégarine est adulte, prête pour l'accouplement. Dès lors on voit réapparaître dans son cytoplasme des formations chromatoïdes un peu différentes des précédentes et dont nous avons parlé déjà précédemment. L'accouplement n'a pas toujours lieu, certaines Grégarines en effet sont attaquées par les phagocytes de l'hôte ou envahies par des Microbes qui arrêtent leur développement et produisent en elles des dégénérescences que nous étudierons dans un chapitre spécial.

M. lumbrici accomplit ainsi tout son cycle évolutif sans pénétrer dans les cellules sexuelles de son hôte; elle se nourrit simplement aux dépens du fluide séminal par osmose. Les 
échanges osmotiques peuvent se faire par toute la surface du corps à travers un épicyte toujours peu épais, ils sont encore facilités par l'extrême minceur de la couche ectoplasmique au fond des sillons épicytaires. Ils sont sans doute aussi plus actifs au niveau du mucron antérieur, mais cet organe est trop réduit pour jouer un rôle bien important dans la nutrition. Chez quelques-unes des espèces que nous allons étudier, au contraire il prend un développement considérable et sert très certainement à la nutrition.

\section{M. agilis (M. agilis Stein p. p.)}

Historique et synonyme. - La première description de cette espèce est due à STEIN (1848); mais cet observateur a confondu sous ce nom deux parasites: l'un, qu'il représente planche IX, fig. 1 et 2 , est fort probablement M. lumbrici Henle, l'autre, (planche IX, fig. 3 et 4), correspond à l'espèce que nous étudions. Nous conservons à cette Grégarine le nom spécifique d'agilis parce qu'il est pour ainsi dire consacré par l'usage. Du reste l'espèce a été très bien décrite et figurée sous ce nom de $M$.agilis Stein par A. Schmid (1854) et surtout par Ruschinupt (1885). Ce dernier a donné (taf. 22, fig. 8) un dessin fort exact du parasite et de son extrémité courbée si caractéristique.

Bütschli (1880-82) (pl. XXXIII, fig. 2 et 3) ne distingue pas cette espèce de $M$. lumbrici, il désigne les deux Grégarines sous le nom de $M$. agilis Stein.

Pfetffer(1891) a observé et représenté quelques stades intracellulaires de cette Grégarine et il l'appelle $M$. agilis.

LABBÉ (1899) confond $M$. agilis Stein et $M$. lumbrici Henle, sous le nom de M. tenax Dujardin. Cuénot (1901), a montré que ce nom de tenax ne devait pas être conservé; mais il ne paraît pas avoir étudié $M$. agilis, cette espèce est cependant très commune et je l'ai rencontrée constamment dans $L$. her'culeus, L. castaneus provenant soit de la Franche-Comté, soit du Dauphiné, soit de la Provence. 
DRZEWECKr (1903) a observé ce parasite auquel il donne le nom de $M$. agilis Stein.

C'est sans doute encore à cette espèce que Brasil (1905 b) a eu affaire ; bien qu'il la dénomme en effet, $M$. lumbrici Henle, il reconnaît n'avoir pas toujours observé le bouquet de poils antérieur (postérieur pour nous) et ajoute "La Grégarine que j'ai le plus souvent vue se rapproche bien davantage de celle que figure Bütschli par exemple (1882, pl. XXXIII, fig. 3 e et $3 \mathrm{f}$ ) que du dessin de Cú́not (1901, pl. XVIII, fig. 2) ». Ces figures de Bütschlr auxquelles il fait allusion représentent en effet très bien certaines formes de notre parasite.

Aucune description absolument précise de cette espèce n'ayant été faite, nous allons en donner une brève diagnose avant de commencer l'étude détaillée de ses caractères.

Corps ayant en moyenne 303 à $350 \mu$ de long sur une trentaine de jo d'épaisseur, fusiforme, le plus souvent courbé en arc; l'une des extrémités est pointue, l'autre généralement infléchie de façon à former un genou sur la convexité duquel le cytoplasme est dépourvu de toute granulation ou inclusion et sur lequel l'ectoplasme est très mince. Stries épicytaires longitudinales fines, fibrilles contractiles peu visibles. Noyau sphérique ne renfermant qu'un seul karyosome.

Houvements très variables, rappelant parfois ceux de $M$. lumbrici Henle, mais non rectilignes, et, le plus souvent aussi, à peine sensibles et n'affectant pas la forme extérieure du corps.

Hotes. -- L. terrestris L., Müller, L. rubellus Hoffm., L. castaneus. Sav.

Habitat et sik̀ge. - M. agilis nous a paru l'espèce la plus répandue chez les Lombriciens; on la trouve couramment en grande abondance dans les vésicules séminales de Lumbricus terrestris L., Müller, L. rubellus Hoffm., L. castaneus Sav. Les premiers stades de son développement se passent dans l'intérieur des blastophores de l'hôte ; puis la Grégarine devient libre au milieu des éléments sexuels qui emplissent les vésicu- 
les séminales, là elle achève de croître, s'accouple et sporule.

Forme, dimensions, houvements. - Le parasite est très polymorphe. Sa forme est en général celle d'un fuseau parfois court, renflé et très régulier, parfois très allongé, peu épais, presque toujours courbé en arc, et avec une des extrémités géniculée.

Les deux pointes du fuseau peuvent se rabattre sur le ventre, et même, d'après Schuidt (1854, pl. XIV, fig. 8) se fusionner ; je n'ai pas observé cette fusion, mais le parasite a souvent l'aspect de la figure 7 du même auteur.

La figure . xxvi nous montre les formes sous lesquelles il se présente le plus communément.

Le parasite adulte peut atteindre jusqu'à $500 \%$ de long sur 40 j. d'épais-

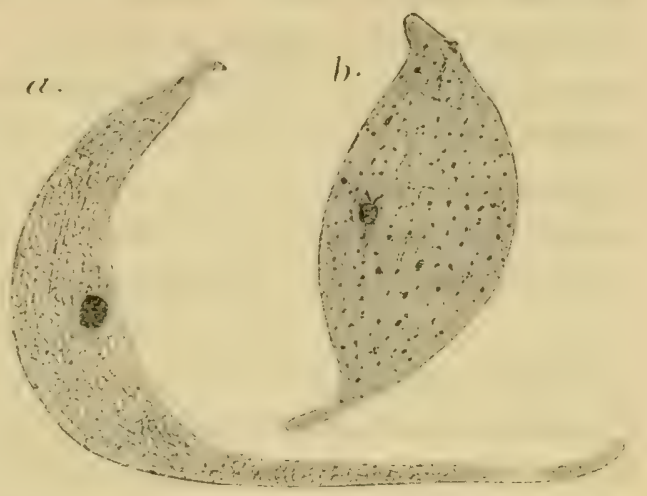

FI⿳. XXVI. M. agilis $\times 200$. Les 2 formes les plus communes. seur, certaines formes plus épaisses ne dépassent pas $350 \mu$ de long et ont jusqu' à $50 \mu$ d'épaisseur.

De même que $M$. lumbrici, $M$. agilis s'observe souvent en mouvement. Schmot (1854) a décrit les particularités de ce mouvement et montré combien il est capricieux. Souvent le parasite paraît complètement immobile et rigide, et l'on peut remarquer avec Schind que le mouvement est toujours moins vif que chez $M$. lumbrici et qu'il ne se produit jamais en ligne droite ; il modifie aussi moins profondément les contours extérieurs du corps. Celui-ci se renfle simplement en une nodosité peu accentuée à l'endroit où se trouve la plus grande accumulation de cytoplasme et cette nodosité se déplace lentement d'une extrémité du corps à l'autre. 
$M$. agilis présente comme $M$. lumbrici une différenciation antérieure qui permet de l'orienter facilement. Cette différenciation n'a pas été remarquée par les premiers observateurs qui ont étudié le parasite; Stein (1848) et Schmidt (1854) n'en font pas mention. Ruschiaupt (1885) a vu que la pointe antérieure du corps se recourbe en hameçon ou en genou ver's le côté concave du parasite, il a noté également que le cytoplasme contenu dans cette région coudée ne contenait jamais ni granulations, ni inclusions ; mais il ne signale pas la diversité d'aspect que peut revêtir cette formation et il ne paraît pas non plus avoir observé le mucron différencié qui en couronne le sommet. Il existe en effet au centre de la convexité du genou un petit soulèvement conique de la surface du corps rempli par un cytoplasme très dense, dépourvu de granulations, et dans lequel on n'aperçoit jamais ni alvéoles, ni grains de paramylon comme dans le reste de l'entocyte. Ce cytoplasme se colore plus intensivement que le reste de l'endoplasme par les colorants cytoplasmiques : en vert, par le Vert lumière, en rouge par l'Eosine, en bleu par le Mann, en rose par le Giemsa; le sommet du cône reste tout à fait incolore ou bien il se colore comme la chromatine (fig. xxvi). Sur toute la surface de ce cône, l'épicyte est très mince; il paraît même manquer au sommet où le cytoplasme serait à nu; les stries épicytaires s'arrêtent à la base du cône où s'interrompt également la couche de sarcocyte (fig. 10). Les formations ectoplasmiques sont aussi aljsentes sür le reste de la bosse, mais là, l'épicyte est toujours un peu plus épais que sur le mucron.

La bosse et son mucron apparaissent lorsque le parasite est encore dans le blastophore nourricier. La région géniculée peut être plus ou moins développée ; parfois elle manque totalement, la Grégarine prenant l'aspect d'un fuseau symétrique ; mais le cône d'endoplasme hyalin est constant, on l'aperçoit toujours au voisinage de l'un des pôles du parasite. C'est ce pôle que nous considérons comme antérieur ; le mucron hyaloplasmique, en effet, de même que celui que nous avons décrit 
chez $M$. lumbrici rappelle les formations épiméritiques plus développées de certaines Grégarines ; il ressemble étonnamment par exemple à l'appendice antérieur de Lankesteria ascidiae R. Lank., que SiedLecki (1899) regarde comme une petite masse de cytoplasme hyalin amœboïde qui ferait hernie à l'extrémité du parasite, et serait destinée à remplacer un épimérite plus développé tombé auparavant.

La fonction de cet appareil est énigmatique; il ne peut pas servir à la fixation; peut-être joue-t-il un rôle dans la nutrition; la minceur de l'ectoplasme qui le recouvre facilitant beaucoup les échanges nutritits ; mais ce rôle est certainement très réduit, presque nul, étant donné le très faible développement de l'appareil ; il est fort probable qu'il s'agit simplement là d'un organe rudimentaire, reste ancestral d'une formation volumineuse, ce qui indiquerait que M.agilis dérive d'une forme fixée (Monocystidée ou Polycystidée) pourvue d'un épimérite bien développé.

On rencontre fréquemment des $M$. agilis très allongées, grêles, et qui sont terminées à l'une des extrémités par un léger renflement en forme de pied (fig. 11); ce renflement correspond sans doute à la bosse des $M$. agilis renflées, le talon étant l'homologue du mucron cônique ; cependant le pied tout entier est rempli par un cytoplasme dense, sans alvéoles ni inclusions et l'on n'observe sur sa surface ni sarcocyte, ni stries épicytaires, en outre la cuticule qui le recouvre est très mince, sa structure est celle du cône mucronaire, il faudrait done homologuer au mucron non seulement le talon, mais le pied tout entier.

Les parasites pourvus d'un genou sont en général plus courts et plus renflés que ceux qui sont munis d'un pied, ceux-ci atteignent jusqu'à $500 \mu$ sur 40 : tent pas plus de $350 \mu$ de long pour $50 \mu$ d'épaisseur et peuvent même être plus trapus encore, comme ceux que l'on rencontre chez $L$. rubellus. La forme géniculée seule existe chez cet hôte, elle est excessivement renflée, j'en ai observé dont l'épaisseur était de $120 \mathrm{j}$, la longueur totale n'excédant pas $145 \%$ 
Le genou est peu marqué, la queue courte, la forme se rapproche de celle d'une sphère. Le parasite est encore courbé en arc. Les deux formes géniculée et pédiculée se rencontrent à la fois chez $L$. terrestris et chez $L$. castaneus; dans L. terrestris, les dimensions du parasite sont beaucoup plus considérables que chez L. rubellus; chez L. castaneus au contraire, elles sont plus réduites.

La cuticule ou épicyte de M.agilis est très mince, légèrement plus épaisse cependant que chez $M$. lumbrici ; elle est ornée de fines stries méridiennes très régulièrement espacées, plus serrées et plus fines chez les formes grêles et allongées à extrémité antérieure pédiculée, plus espacées et plus marquées chez les formes courtes et épaisses à extrémité antérieure géniculée. Ces stries sont plus rapprochées que chez $M$. lumbrici; il n'existe pas ici comme chez cette dernière espèce de sillons profonds entamant les couches sous-jacentes de l'ectoplasme.Les stries épicytaires ne se continuent pas sur la bosse et le mucron ou sur le pied, mais elles sont visibles sur tout le reste du corps (fig. $\mathrm{xxvI}$ ).

De même que l'épicyte, l'ensemble de l'ectoplasme est aussi plus développé que chez $M$. lumbrici. Entre la cuticule et le sarcocyte, on observe souvent une zone claire qui correspond peut-être à la "Gallertschicht " décrite par Schewiakoff (1894) chez Clepsidrina munieri; mais sa présence n'est pas constante.

Le sarcocyte assez fortement coloré par les colorants cytoplasmiques et de consistance en apparence homogène offre la même épaisseur que la couche claire; cette épaisseur est uniforme sur toute la surface du corps, sauf sur le genou et son mucron, ou sur le pied où le sarcocyte manque toujours.

Les stries du myocyte sont beaucoup moins apparentes que chez $M$. lumbrici, elles sont parallèles, excessivement fines, très serrées, beaucoup plus encore que les stries épicytaires. Il faut des colorations appropriées pour les mettre en évidence ; le Mann et la méthode de Benda pour la recherche des mito- 
chondies nous ont donné les meilleurs résultats à ce point de vue ; avec les méthodes ordinaires, on arrive cependant à apercevoir ces fibrilles en coupe optique, elles se présentent sous forme de grains colorés excessivement ténus et très rapprochés qui forment une zone entre le sarcocyto et l'entocyte (fig. 11).

L'entocyte est constitué par un cytoplasme finement granuleux dans lequel sont baignés les grains de paramylon et les granulations chromidiales.

Le cytoplasme est plus dense que dans l'espèce précédente, il est creusé d'alvéoles très petites, dont quelques-unes s'agrandissent considérablement pour loger les grains de réserve. Les travées de spongioplasme sont plus ou moins épaisses suivant le développement de ces grains. On y distingue deux sortes de granulations, les unes se teintent par les colorants chromatiques : en bleu foncé par l'Hématoxyline, en violet par le Giemsa ; les autres sont imprégnés par les colorants plasmatiques, elles prennent, traitées par le Giemsa l'aspect de fines gouttelettes rose pâle. La coloration du cytoplasme est variable d'ailleurs avec les stades du développement : dans les formes jeunes il est éosinophile et il prend une teinte bleue ou violette par le Giemsa; dans les parasites adultes prêts à l'accouplement, il devient orangophile et, par le Giemsa, se colore en rouge.

Comme chez $M$. lumbrici, le réseau spongioplasmique se dilate en certains points où il change également de structure; au lieu d'être granuleux, il semble alors formé d'une substance fluide qui prend avec une grande intensité les colorants cytoplasmiques et dans laquelle s'observent à certains moments les chromidies qui sont parfois excessivement nombreuses, surtout dans le voisinage du noyau, mais qui cependant sont généralement moins abondantes que chez $M$. lumbrici. Il existe ici aussi deux sortes de ces formations chromatoïdes, les unes précédant l'apparition des grains de paramylon, les autres se montrant au moment où ces grains sont en voie d'absorption.

Parmi les premières il en est qui se présentent sous forme $\triangle R C H, D E$ ZOOI, EXP. ET GEN, - 5e SERIE, - T. III. - (II). 
de masses irrégulières très volumineuses et qui rappellent beaucoup l'aspect du karyosome, elles ont été vues déjà par DrzeWECKI (1903), nous en reparlerons ultérieurement. D'autres plus petites proviennent peut-être de l'émiettement des précédentes, ce sont des grains sphériques ou irréguliers, des bâtonnets, des anneaux qui restent isolés ou se rassemblent en figures régulières.

Les chromidies qui apparaissent au moment où les grains de paramylon sont en voie de résorption sont de deux sortes : les

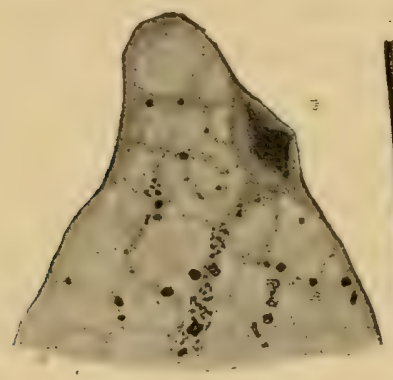

$X X V I 6$

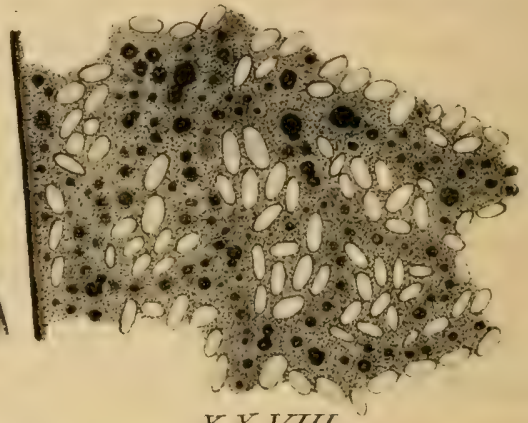

XX IIII.

FIG. XXVII. Mon. agilis. Mucron très coloré. Grains chromidiaux irréguliers dans les travées fortement colorées du spongioplasme, $\times 1.300$. - FIa. XXVIII. $M$. agilis. Absorption des réserves. Masses chromatoïdes sphériques dans le cytoplasme $\times \mathbf{1 . 3 0 0}$.

unes sont de petites granulations irrégulières (fig. XxvII) ayant l'aspect des grains chromatiques nucléaires ; les autres, toujours assez régulières, sont sphériques ou formées de deux sphères accolées de diamètre inégal (fig. xxviII) elles sont renfermées dans une zone sphérique également plus colorée que le cytoplasme environnant; celui-ci présente aussi plus d'affinité pour les colorants chromatiques de sorte que les nœuds du réseau où se trouvent parfois accumulées en certaine quantité les granulations chromatoïdes tranchent fortement sur le reste du cytoplasme et simulent des masses multinuclées à contour amiboïde qui pourraient facilement être confondues avec des parasites (fig. 18).

Les grains de réserve sont très variables comme forme et 
comme dimensions, on en observe qui n'ont que 1 \%. 5 à $2 \%$ d'épaisseur sur 4 à 5 „ de long; d'autres au contraire sont sphériques et atteignent 7 à 8 y. de diamètre, ceux-ci ne sont jamais très nombreux dans les parasites normaux, on peut en voir quelques-uns de ce volume dans le voisinage de la queue (fig. 14) tous les autres étant de taille normale, soit, comme chez $M$. lumbrici, $3 \mu$ sur 6 \% en moyenne.

Le noyau est logé vers le milieu du corps, il occupe généralement la région la plus renflée du fuseau et peut être situé au voisinage de l'axe ou tangent à la surface concave du parasite. C'est une vésicule sphérique ou ellipsoïdale, limitée par une membrane mince peu avide des colorants chromatiques, et qui renferme en son centre un karyosome assez volumineux. Il peut exister plusieurs karyosomes, mais c'est une exception : nous avons ainsi rencontré un karyosome émietté en un grand nombre de grains chromatiques vacuolaires (fig. 12). De ce karyosome irradient des filaments de linine qui sont disposés en un réseau sur lequel sont placés quelques grains chromatiques de dimensions variables, mais en général peu volumineux. Les mailles du réseau sont remplies par un suc nucléaire qui se teint avec intensité par les colorants plasmatiques et dans lequel on observe parfois une sorte de coagulum granuleux.

Le karyosome a toujours une forme voisine de celle de la sphère, mais sa surface est le plus souvent, rugueuse, mamelonnée; elle paraît servir de point d'attache aux filaments du réseau lininien.

Coloré par l'Hémalun, le Fer, la Safranine, le rouge Magenta, le Carmin, le karyosome se présente comme une masse assez foncée, parfois massive, parfois creusée do vacuoles et dans laquelle, il n'apparaît pas de différenciation. Avec la méthode de Benda pour la recherche des mitochondries le karyosome paraît quelquefois plus foncé au centre; autour de cette masse centrale violette, on aperçoit une zone rouge creusée de vacuoles; à la périphérie se trouve une sorte de croûte mince colorée en violet foncé comme la masse centrale. La méthode 
de Mann met également en évidence une enveloppe homogène peu épaisse, colorée en violet, (fig. 171, 173, 176) alors que le reste du' karyosome est rouge et creusé d'assez nombreuses vacuoles.

Telle est la structure normale du noyau, mais nous verrons en étudiant le développement, que cette structure est très instable et qu'elle varie à chaque instant au cours de la vie végétative.

De même que pour $M I$. Tumbrici, nous n'avons pas réussi à

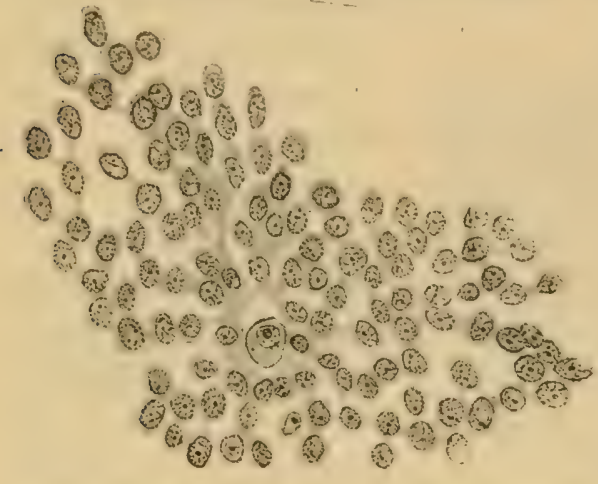

Frg. XXIX. $M$-agilis jeune dans un blastophore entouré de spermatocytes I $\times 1200$. distinguer les kystes et les sporocytes de M. agilis.

Nous étudierons dans un chapitre spécial la déhiscence des sporocystes qui s'ouvrent dans l'intestin ainsi que la forme et les mouvements du sporozoïte. Nous n'avons pas vu la migration de ce sporozoïte

à travers la paroi intestinale et nous ne savons pas par quelle voie il gagne les vésicules séminales. Dans celles-ci, les plus jeunes stades que nous ayons observés étaient déjà logés dans les spermatosphères de l'hôte.

Ce sont des corps ovoïdes ou ellipsoïdaux, parfois sphériques ayant 7 à $8 \mu$ de long sur 4 à $5 \mu$ d'épaisseur, c'est-à-dire à peine plus volumineux que les sporozoïtes, nous en avons même remarqué quelquefois ront les dimensions étaient moindres encore, $4 \mu 5$ sur $3 \mu .75$ (fig. Xxix, fig. 107, 108, 109).

La pénétration a toujours lieu lorsque la spermatosphère est au début de son évolution ; elle se produit parfois même dès l'instant où le follicule tombe du testicule, avant la phase de dissociation de cefollicule. Cette pénétration semble être pour ainsi dire passive; le parasite insinué entre les pédicules 
de deux cellules germinales voisines et logé près du centre de la spermatosphère est entouré par le cytoplasme résiduel qui provient des cellules mâles en voie de développement et qui doit constituer le blastophore (fig. 109). Je n'ai jamais vu de $M$. agilis très jeune dans des blastophores adultes. Les figures 21 et 22 planche XIV de Schmidt (1854) où la très jeune Grégarine est placée dans un blastophore sphérique volumineux, représentent sans doute les premiers stades d'une autre espèce, $R$. pilosa que nous étudierons ultérieurement, il en est de même des figures 37 et 38 , planche XXII de RuschHaupt (1885). Schmid etRuschiaupt supposent que la spore pénètre dans le blastophore et donne directement la jeune Grégarine en perdant son enveloppe; RuschHaupt croit même avoir vu quelques stades de cette transformation qu'il représente fig. 31 et 32. Les connaissances incomplètes que l'on possédait alors sur la constitution du sporocyste et sur les premières phases du développement des Grégarines expliquent facilement l'erreur de ces observateurs. DrzewECKI (1904) ne semble pas avoir vu de stades bien jeunes; sans doute il dit " Die Sporozoïten, mit deutlichen Nucleolus versehen, dringen in ein Blastophor ein, runden sich ab, etc. ") ; mais dans ses figures $1,2,3 \mathrm{a}, 3 \mathrm{~b}$, planche 9 , qui représentent cependant d'après lui des sporozoïtes dans les blastophores, on ne voit pas trace de parasites. Dans la figure 4, c'est bien un $M$. agilis qui cette fois est dessiné, mais c'est un individu presque adulte.

Les figures de Schmid et de RuschHaupt donnent une idée assez exacte de la forme du jeune parasite, mais les détails de structure n'y sont pas relevés.

En général, il n'y a qu'un parasite par blastophore, parfois cependant on en trouve deux dans le même blastophore (fig. $\mathrm{xxx}$ ).

La plus petite Grégarine que nous ayons rencontrée mesurait ainsi que nous l'avons dit $4 \mu .5$ sur $3 \mu .75$. Son cytoplasme finement granuleux était très dense, sans vacuoles, ni inclusions. Le noyau légèrement excentrique de 1 ;. 5 de diamètre 
renfermait un karyosome de 0 \%. 75 entouré par une zone claire. Il n'y avait ni réseau de linine, ni grains chromatiques visibles.

Les parasites un peu plus volumineux gardent la même forme ovoïde ou sub-sphérique $7 \mu 5$ sur $4 \mu, 5,7 \mu .5$ sur $5 \mu$, 7 . 5 sur 6 . 6 . Mais leur cytoplasme, toujours finement granu-

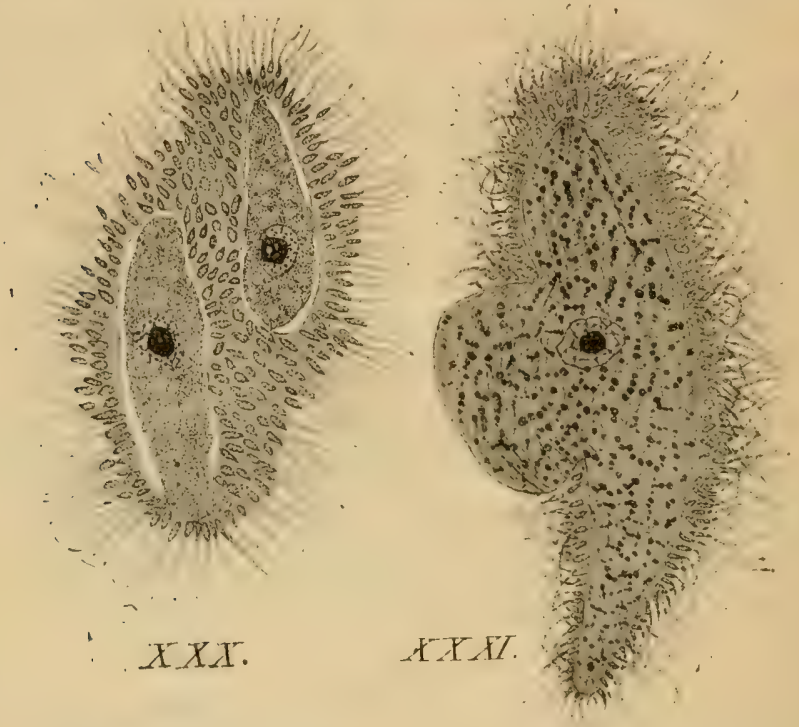

FIG. XXX. Deux $\boldsymbol{M}$, agilis dans un même blastophore $\times 650 .-$ FIG. XXXI. $\boldsymbol{M}$. agilis dans 1 blasphore $\times 650$. Le milieu du corps fait hernie hors de la cellule hôte.

leux paraît moins dense; dans la région antérieure, en avant du noyau, on voit apparaître quelques vacuoles, l'une d'elles, plus développée et constante occupe toujours le pôle antérieur (fig. 108). C'est peut-être là l'origine du cône mucronaire, malheureusement je n'ai pu suivre sa destinée au cours du développement de la Grégarine. On aperçoit parfois ça et là dans l'entocyte quelques granulations sidérophiles peu volumineuses que le Mann colore en rouge de même que le karyosome (fig. 171). Le noyau sphérique mesure de $2 \mu 5$ à $3 \mu$; il est entouré d'une membrane peu chromatique et l'on voit en son centre un volumineux karyosome, sphérique également, et qui a de 1 « 25 à $2 \%$ de diamètre. 
Ce karyosome est coloré en gris pâle par le Fer, en rouge par le Mann; cette dernière méthode décèle à sa surface une zone mince colorée en violet et une ou deux petites vacuoles dans l'intérieur (fig. 176). Autour de ce karyosome rayonnent les filaments de linine que met en évidence le Mann; ils sont colorés en bleu pâle et l'on peut voir, accolés à leur surface les grains chromatiques violets ou bleu foncé. Ces grains peuvent être dispersés sans ordre apparent sur le réseau de linine; mais en général, on les voit disposés en deux cercles réguliers, l'un à une faible distance du karyosome (fig. 108, 110), l'autre, formé par les grains accolés à la membrane. Le Fer ne met pas bien en évidence le réseau de linine; avec cette méthode, on ne voit en général que le karyosome avec les deux cercles concentriques de grains chromatiques, le cercle le plus proche du centre étant séparé du karyosome par une zone claire plus ou moins large.

Dans ces stades jeunes, les rapports des dimensions linéaires du parasite, du noyau et du karyosome peuvent être à peu près représentés par les chiffres $1, \frac{2}{5}$ et $\frac{1}{5}$. Quelquefois, le noyau est plus volumineux par rapport au corps, et le karyosome par contre, beaucoup plus petit; nous avons représenté (fig. 112) une jeune $M$. agilis de $8 \%$ de diamètre qui montre un volumineux noyau de $5 \mu$ de diamètre avec un petit karyosome vacuolaire de $1 \mu .5$ de diamètre. Il s'agit là, vraisemblablement d'une forme pathologique. L'hypertrophie du noyau est due à un grand développement du suc nucléaire; ces noyaux très grands sont en effet pâles et paraissent peu chromatiques. Le réseau de linine $\mathrm{y}$ est très net, les grains de chromatine, peu nombreux sont disposés sur un cercle entourant le karyosome, quelques-uns sont disséminés sur le réseau entre ce cercle et la membrane, enfin, il en est d'autres, plus volumineux qui sont accolés à cette membrane.

Le parasite grandit en conservant d'abord sa forme ovoïde ou sphérique; on en observe qui ont de 9 à $15 \%$ de long sur 7 à $10 \mu$ d'épaisseur ; la croissance devient ensuite plus rapide suivant le grand axe et on peut noter les dimensions suivantes : 
$16 \mu$ sur $12 \mu, 19 \mu$ sur $15 \mu, 19 \mu .5$ sur $10 \mu, 30 \mu$ sur $12 \mu$, $40 \mu 5$ sur $13 \mu$; le parasite devient ainsi un ovoïde allongé ou un ellipsoïde (fig. 110, 111); puis, ses extrémités s'étirant en pointe, un fuseau plus ou moins régulier. L'extrémité antérieure se coude, se repliant plus ou moins complètement sur le reste du corps, et constitue le genou de l'adulte. On voit alors le mucron conique sur sa convexité. Souvent la pointe postérieure se reploie également et l'ensemble garde une forme voisine de la sphère en distendant considérablement la cellule hôte; ce reploiement peut ne pas se produire, alors la cellule hôte se déforme en se distendant et épouse la forme en fuseau du parasite.

Celui-ci est recouvert par une sorte de toison constituée par la membrane du blastophore portant son revêtement de cellules séminales plus ou moins profondément altérées et qui seront décrites plus loin (fig. xxxI). Bientôt, la croissance continuant, cette enveloppe blastophorienne se rompt, le parasite s'échappe et devient libre dans les vésicules séminales. La présence de cette enveloppe velue avaitété notée déjà par les anciens observateurs; STEIN (1848) représente ainsi (fig. 4, planche IX), une jeune $M$. agitis dans un blastophore; LIEBERKÜHN (1855) figure aussi plusieurs Grégarines velues (fig. 3 et 7 , planche I et fig. 7, planche VI), qui sont également des Mon. agilis contenues dans des blastophores à divers stades. Pour ces auteurs, l'enveloppe faisait partie intégrante du corps du parasite et LIEBERKÜHN notait que ces parasites étaient sujets à des mues et pouvaient se débarrasser de cette enveloppe. SCHNEIDER (1875) ne croit pas à la réalité de cette mue, mais il n'explique pas le phénomène; comme l'a fait observer BaLbiani (1884), c'est Schмпт (1854) qui, le premier a montré la nature de la toison enveloppante et expliqué ainsi les mues; il a représenté des Monocystis en train de sortir de blastophores altérés.

Généralement le parasite dégage d'abord l'une de ses extrémités ; mais il peut arriver aussi que ce soit vers le milieu de son corps que se fasse la rupture du blastophore; c'est alors 
la partie médiane du parasite qui sort la première de l'enveloppe; notre figure xxxi montre une Grégarine dont le ventre fait ainsi hernie ce qui lui donne un aspect tout particulier. Après Schmid, Ruschinupt (1885), et Drzewecki (1903) ont remarqué les cellules séminales altérées qui recouvrent les jeunes $M$. agilis ; mais ils n'ont pas vu aussi exactement que lui la façon dont le parasite s'en débarrasse. RuschHaUpT pense que la Grégarine est parfois forcée de s'enkyster dans l'enveloppe blastophorienne, et qu'alors le revêtement de spermatozoïdes disparaît peu à peu à partir du pôle antérieur. Pour Drzewecki aussi, les spermatozoïdes tombent peu à peu. Nous n'avons jamais observé ce fait; toutes les Grégarines qui sortent de l'enveloppe blastophorienne amènent la rupture de cette enveloppe et sortent par la fente ainsi produite, comme l'ont remarqué Lieberkühn et Schmidt. Cette sortie a lieu en général lorsque le parasite a atteint presque son complet développement, cependant elle peut se produire avant; nous avons vu par exemple des jeunes Grégarines de $12 \%$ sur 10 « 5 sortir du blastophore nourricier et d'autres de cette taille ou un peu plus grandes déjà dépouillées de leur enveloppe. Nous n'avons pu établir comment se fait dans ces conditions la rupture de la cellule hôte.

Le volume du noyau grandit d'abord proportionnellement à celui du corps ; puis il s'accroît plus lentement. Les rapports entre les dimensions du noyau et celles du karyosome varient également. Le karyosome présente longtemps des dimensions moitié moindres que celles du noyau ; puis le noyau s'accroît plus rapidement et, chez les Grégarines adultes, nous pouvons noter les dimensions suivantes pour les parasites, leur noyau et leur karyosome.

\begin{tabular}{|c|c|c|c|}
\hline \multicolumn{2}{|r|}{ Parasites } & Noyaux & Karyosomes \\
\hline 19 & ․ 5 sur $10 \mu .5$ & $7 \mu .5$ & $3 \mu 10$ \\
\hline 40 & $\mu . \quad \operatorname{sur} 13 \mu$ & $7 \mu$ & $4 \%$ \\
\hline 300 & sur $33 \mu$ & $22 \mu .5$ sur $10 \mu .5$ & $7 \mu .5 \operatorname{sur} 6 \mu$ \\
\hline 435 & sur $27 \mu$ & $33 \mu . \operatorname{sur} 7 \mu .5$ & $7 \mu \cdot 5 \operatorname{sur} 4 \mu$ \\
\hline
\end{tabular}


A mesure que s'accroît le parasite, la compacité de son cytoplasme diminue; nous avons vu apparaître à son pôle antérieur une vacuole claire qui est peut-être le point de départ de la formation mucronaire; mais je ne puis être affirmatif sur ce point car je n'ai pu suivre son évolution. Le reste de l'entocyte ne tarde pas à se creuser d'alvéoles dont les dimensions vont en augmentant et dans lesquelles apparaissent les grains de réserve. Les granulations chromidiales se montrent de très bonne heure sous forme de petits grains ou de sphérules isolés ou rassemblés en masses un peu plus volumineuses; elles se colorent fortement par le Fer; le Mann leur donne une teinte rouge. Nous allons voir qu'elles sont probablement d'origine nucléaire.

Lorsque les grains de paramylon sont bien développés, les chromidies disparaissent complètement; puis il ne tarde pas à en apparaître d'autres qui sont, celles-ci, manifestement d'origine nucléaire et dont nous reparlerons un peu plus loin.

L'évolution du noyau est assez compliquée. Drzewecki a étudié (1904) en détail les échanges qui se produisent entre le noyau et le cytoplasme au cours de la vie végétative du parasite et il déclare avoir assisté à des disparitions et à des réapparitions successives du noyau et des grains de paramylon. Il nous a été impossible de revoir ces phénomènes en ce qui concerne les grains de paramylon; quant au noyau nous allons décrire son évolution.

Nous n'avons jamais observé de disparition du noyau durant les premiers stades du développement. DRzewecki a décrit des faits de ce genre, mais ses figures sont loin d'emporter la conviction; on peut facilement se convaincre que cet observateur n'a pas observé de stades très jeunes du parasite, il suffit pour cela de comparer les premières figures, planche IX de son travail avec nos figures 107, 108, 109, 110 ou avec celles de Schmid et de RuschHaupt. On ne voit pas de parasites dans les blastophores représentés par DRzEWECKI à moins qu'il ne s'agisse là d'une véritable " mycoplasmose " comme 
on en a signalé chez certaines cellules végétales parasitées par des Urédinées.

A part quelques cas pathologiques signalés précédemment, les formes jeunes ont toujours un noyau normal arrondi, avec karyosome central et grains chromatiques sur un réseau. Dès ce moment, on peut observer, comme chez $M$. lumbrici des figures qui font penser à un bourgeonnement du karyosome; la figure 176 montre un karyosome de jeune $M$. agilis dans le voisinage duquel on observe un grain rouge très rapproché; dans la figure xxxII on voit également un grain assez volumineux encore rattaché au karyosome par un mince pédicule. Dans les figures 16 et 17 nous avons représenté des karyosomes desquels paraissent s'échapper une série de filaments radiaires sur lesquels sont échelonnés des grains fortement colorés de diverses grosseurs. De nombreux observateurs ont remarqué ces grains qui paraissent s'échapper du karyosome. BERndT (1902) les a signalés chez les Grégarines de la larve de Tenebrio molitor et décrit leur mode de sortie que KUSCHAKEWITSCH (1907) a observé à son tour; nous n'avons pas retrouvé le même processus chez $M$. agilis. LÉGER et DuboscQ (1904) ont distingué chez Stylorhynchus deux sortes de ces grains bourgeonnés, les plus petits sont basophiles, les plus grands acidophiles. Chez $M$. agilis les petits grains bourgeonnés nous ont toujours montré les mêmes affinités colorantes que le karyosome, avec le Mann par exemple, ils se colorent en rouge. Mais on rencontre une autre modalité de ce bourgeonnement; dans la figure 174 (préparation colorée à la méthode de Mann) on peut voir le karyosome volumineux excentrique dont un hémisphère tangent à la membrane nucléaire est coloré en violet comme la chromatine, tandis que l'autre moitié tournée vers le centre est colorée en rouge, toutes les deux étant également vacuolaires; dans la figure 172 le karyosome rouge paraît comprimé par trois gros grains violets accolés à sa surface.

Ces karyosomes formés de deux parties à colorabilité diffé- 
rente ont été décrits aussi par DoGiel (1906) chez Cystobia chirollotae ; Kuschikewitch (1907) les a rencontrés également chez Gregarina polymorpha. Ces observateurs représentent de tels karyosomes dont une partie fait saillie dans le cytoplasme, en bosselant la membrane nucléaire et leurs figures rappellent dans une certaine mesure notre figure 174. NERESHEIMER (1905) a trouvé aussi chez Amoba Dofleini des noyaux
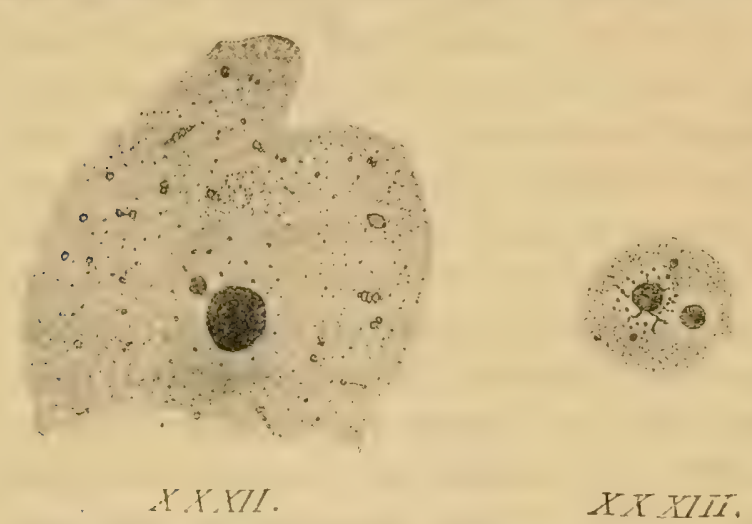

Fic. XXXIT, $M$, agilis $\times 1300$. Région antérieure d'une forme massive. Dśbut du noyau flammé : membrane nucléaire avec 2 bosses situées à deux pôles diamétralement opposés. 1 grain bourgeonné encore rattaché au karyosome. Masses chromidiales dans le cytoplasme. - FIG. XXXIII. Jeune M. agilis $\times 1300$. Dans le cytoplasme, une masse chromatoïde entourée d'une zone claire.

dans lesquels le karyosome donne par bourgeonnement un nucléole à colorabilité différente; celui-ci se détache du karyosome puis il passe dans le cytoplasme où il ne tarde pas à disparaître. Il est fort possible que chez $M$. agitis les masses basophiles qui bossellent la surface du karyosome se détachent de celui-ci pour tomber dans le suc nucléaire et même dans le cytoplasme, mais nous n'avons pas observé cette chute.

Pendant que se déroulent ces processus, la membrane nucléaire reste intacte, et il semble difficile qu'elle puisse livrer passage à des masses solides comme des grains de chromatine ; cependant le cytoplasme se remplit peu à peu de granulations qui présentent beaucoup d'affinité pour les colorants chromatiques; les unes sont de très faible volume, sphériques ou irrégulières, ainsi que nous l'avons vu, elles peuvent se rassembler en bâtonnets ou en figures régulières (fig. xxxII), on les 
aperçoit disséminées dans toute la masse cytoplasmique; les autres sont très volumineuses, elles atteignent et même souvent dépassent la taille du karyosome. Drzewecki (1903) en a décrit qui ont absolument l'aspect de noyaux, elles sont situées au milieu d'une zone claire et entourées par une membrane différenciée. Je n'ai pas réussi à retrouver de ces masses ainsi encerclées d'une membrane, bien que j'en aie observé assez fréquemment. Je les ai toujours vues au centre d'une zone claire dépourvue de membrane, leur aspect rappelle beaucoup celui du karyosome (fig. xxxIII). Leur colorabilité comme leur volume sont très variables. Avec l'Hématoxyline ferrique, elles se comportent toujours comme le karyosome; avec le Mann, il n'en est pas de même, parfois elles prennent la coloration rouge du karyosome et sont comme lui vacuolaires, présentant une mince couche colorée en violet à leur surface (fig. 171) ; mais elles peuvent aussi se colorer en bleu foncé ou en violet (fig. 172 et 174) comme la chromatine du réseau nucléaire. On trouve souvent deux do ces masses, l'une en avant du noyau, l'autre en arrière (fig. 111), parfois une seule dans le voisinage du noyau, parfois 3 ou 4 dispersées dans le cytoplasme. En général, les masses les plus volumineuses sont colorées en violet par le Mann, les plus petites se colorant au contraire en rouge.

Ces chromidies sont connues depuis longtemps chez les Grégarines, Schneider (1875) les avait déjà signalées; depuis elles ont été revues par nombre d'auteurs, DrzeweCki (1903), LÉger (1904, 1906, 1907), LÉger et DuboscQ (1902, 1904). Brass (1883-84), et KusChaKEwITSCI (1907) ont trouvé chez diverses Gregarina des masses aussi volumineuses que celles que nous venons de décrire chez $M$. agilis : nous avons vu que Drzewecki les a également observées chez cette même espèce.

KUSCHAKEWITSCH (1907) pense queces masses proviennent de l'agglomération des petites granulations chromidiales disséminées un peu partout dans le cytoplasme; il n'mdique pas leur sort ultérieur. Drzewecki (1903) croit qu'elles s'émiet- 
tent en grains plus petits, puis finalement se dissolvent dans le cytoplasme.

La plupart des auteurs considèrent ces masses chromatoïdes comme d'origine nucléaire; pour DRZEWECKI, au contraire, elles seraient dues à l'abondance de matières nutritives, et chez Stomatophora coronate dont nous reparlerons plus loin, il les considère comme de la chromatine de néo-formation qui se constituerait dans le plasma aux dépens des substances nutritives absorbées. Nous n'avons rien observé de certain sur la genèse de ces productions, cependant, nous sommes porté à croire qu'elles ont bien une origine nucléaire. Nous avons décrit plus haut des parasites chez lesquels le karyosome est formé de deux parties à colorabilité différente dont l'une, accolée à la membrane nucléaire refoule celle-ci devant elle, faisant ainsi en quelque sorte hernie dans le eytoplasme. C'est cette partie qui, se détachant du karyosome et tombant dans le cytoplasme donnerait une masse chromidiale volumineuse. S'émiettant ensuite en fragments plus petits, cette masse donne naissance à une série de granulations sidérophiles qui se dispersent dans le plasma. Ces granulations jouent sans doute un rôle dans la formation des grains de réserve, comme le supposent LÉGER et Duboscq (1908). Ainsi que Drzewecki l'avait déjà remarqué, les parasites qui renferment les grosses masses chromidiales sont dépourvus de grains de paramylon ou du moins ne renferment que des grains très petits et peu nombreux; à mesure que ceux-ci grossissent et se multiplient, les granulations chromidiales diminuent. La Grégarine adulte n'en renferme plus guère; c'est un sac bourré de grains de paramylon de forme et de taille variées et au milieu duquel se trouve le noyau. Ce stade dure peu. Le noyau jusque là sphérique ou ovoïde présente bientôt à l'un des pôles une bosse qui s'étire en pointe ; une bosse semblable apparaît au pôle diamétralement opposé, qui s'effile également (fig. xxxıi).

Le noyau a pris la forme d'un croissant, il se place alors en croix avec la direction du grande axe du parasite, ou du moins 
il forme un angle plus ou moins grand avec celui-ci, et l'une des pointes se rapproche de la surface du parasite et arrive même à son contact en un point où se produit une légère invagination. Pendant ce temps, la membrane nucléaire a disparu en plusieurs points par lesquels le suc nucléaire communique directement avec le cytoplasme dans lequel il envoie même des prolongements étroits ; le noyau prend une forme étoilée (fig. 16) c'est le " noyau flammé " de Wolters (1891); nous avons dit déjà à propos de $M$. lumbrici que Cú́Not le considère comme un " artefact ", mais que DrzewEckI a pu l'observer in-vivo. Par les trous de la membrane, les grains bourgeonnés par le karyosome passent directement dans le cytoplasme (fig. 16) ; tout autour du noyau, on peut voir alors dans ce cytoplasme (fig. 18) de larges travées de spongioplasme chargées de granulations sidérophiles qui se dispersent ensuite dans toute l'étendue du corps. Chez les Aggregata, LÉGER et DuBoscQ (1908) ont observé cette émission de grains nucléolaires dans le cytoplasme, et comme elle a lieu au moment de la première mitose, ils pensent que ces grains pourraient bien jouer le rôle de ferments provoquant la dissolution des réserves et leur assimilation. C'est bien ce que l'on observe chez M. agilis. Au moment de l'émission des grains nucléolaires dans le cytoplasme, celuici est bourré de grains de paramylon; ces grains semblent disparaître ensuite peu à peu, et le cytoplasme est alors formé de granulations de diverses grosseurs constituant de larges travées séparant des alvéoles claires où l'on n'aperçoit plus de grains de réserve (fig. 17).

Les grains de paramylon se présentent normalement comme des sphères ou des ellipsoïdes ayant en leur centre une petite vacuole sphérique ou allongée et irrégulière qui se colore fortement par la Safranine ou le rouge Magenta; ces grains sont formés de couches concentriques mais ils sont si compacts que ces couches sont difficilement visibles. Lorsque les grains sont en voie d'assimilation, ils se gonflent fortement (fig. 74) la vacuole centrale s'agrandit considérable- 
ment, et les couches concentriques apparaissent avec une grande netteté, à ce moment le grain s'imprègne fortement par l'Orange G.Je ne sais comment se poursuivent les phénomènes de disparition du grain; après les stades précédents on n'observe plus que des vacuoles parfois fortement colorables par l'Orange, parfois transparentes. Pendant que se produisent ces phénomènes, on voit apparaître dans le réseau spongioplasmique les formations chromatoïdes sphériques que nous avons décrites précédemment, et dont la forme rappelle étonnamment celles que Comes (1907) a observées chez Stylorhynchus longicollis et qu'il représente fig. 18, pl. 20. Pour cet auteur, ces granulations seraient homologuables aux granulations chromatiques du cytoplasme ovulaire et n'auraient pas du tout une origine nucléaire. Il se pourrait qu'elles proviennent de la transformation des grains de réserve et qu'elles soient en quelque sorte un stade de leur digestion. Mais c'est un point que nous n'avons pas cherché à élucider; il nécessiterait des recherches chimiques approfondies. Quoi qu'il en soit nous ne partageons pas complètement l'avis de Comes; si ces granulations ne tirent pas directement naissance du noyau, celui-ci joue tout au moins un rôle indirect important dans leur formation.

Ces granulations sont surtout bien mises en évidence par des colorations à l'Hématoxyline ferrique après fixation au Flemming ou au Benda; même elles paraissent beaucoup plus nombreuses après l'action de ces fixateurs qu'après le sublimé alcoolique ou acétique. Peut-être s'agit-il là de précipités produits par l'agent fixateur. La figure xxxr représente une Grégarine ainsi fixée au Benda et colorée par le Fer. Les masses chromatoïdes ont ici une teinte foncée, il arrive assez fréquemment qu'elles ne se colorent pas tout à fait comme la chromatine, mais qu'elles prennent une coloration brun acajou, plus ou moins claire. Leur affinité pour les colorants chromatiques 's'atténue ensuite, en même temps que leur volume diminue et bientôt, elles no se distinguent plus des autres granulations 
cytoplasmiques que par des dimensions un peu plus considérables (fig. 17).

Le " noyau flammé » est difficile à fixer d'une façon satisfaisante, au moment où il envoie des grains chromidiaux dans le cytoplasme, surtout vers la fin du processus, lorsque le karyosome a perdu en partie sa colorabilité ; il semble même souvent disparaître totalement; mais nous n'avons constaté que très rarement de telles disparitions totales du noyau sur du matériel bien fixé par le Flemming fort; et dans co cas, il s'agit manifestement d'altérations pathologiques; les parasites dépourvus de noyau nous ont toujours montré des signes de dégénérescence.

Parfois le karyosome perd presque toute affinité pour les colorants nucléaires (fig. 18), et comme la membrane, le réseau de linine et la chromatine ont aussi disparu du noyau, celui-ci semble s'être totalement évanoui; mais un examen attentif permet de retrouver au sein du cytoplasme une zone plus colorée au centre de laquelle on observe une masse homogène teintée par les colorants cytoplasmiques et qui est le karyosome devenu achromatique. Le Mann le colore encore en rouge comme le karyosome normal, mais ses contours sont diffus et ne prétentent plus la bordure violette caractéristique (fig. 175). Sans doute, le réseau de linine persiste également, mais il n'est pas démontrable. Du reste, il semble parfois manquer même dans les noyaux normaux (fig. 12 et 173) sans doute parce qu'il n'a pas été mis en évidence par une coloration appropriée.

Après cette sorte de crise, le karyosome récupère sa colorabilité, mais je n'ai pu déterminer par quel processus.

Je n'ai pas rencontré comme RuschHaupt de Grégarines enkystées encore recouvertes de leur enveloppe blastophorienne. $M$. agilis sort du blastophore nourricier avant l'accouplement. En général, elle commence par dégager une de ses pointes, comme l'avaient remarqué Schndт (1854) et LieberKühN (1855) ; quelquefois cependant, c'est la région équatoriale qui 
sort en premier lieu, le parasite se présentant alors avec un ventre renflé qui fait hernie hors du blastophore (fig. xxxr).

Le processus de résorption des réserves relaté plus haut a lieu après cette sortie du blastophore, puis les parasites s'accouplent. Les phénomènes de l'accouplement, de la sécrétion de l'enveloppe kystique ont été décrits par Henneguy (1888), et ceux de la formation des gamètes, de leur accouplement et du développement des sporocystes par Cuénot (1901) Prowazek (1902), Brasil (1905 b), Hoffuman (1908); nous n'y reviendrons done pas ici. Disons seulement que les enkystements solitaires que nous avons pu observer nous ont toujours paru suivis de dégénérescence.

Monocystis striata n. sp.

J'ai observé quelquefois dans les vésicules séminales de Lumbricus terrestris L., Müll., une espèce de Monocystis remarquable par son aspect rigide et par les sillons profonds et les côtes très développées qui ornent la surface de son corps. ('ette Grégarine n'a pas été décrite par les divers auteurs qui ont étudié les Monocystis des Lombrics, je lui donnerai le nom de $M$. striata qui rappelle son caractère le plus saillant.

Voici la diagnose de ce nouveau parasite :

Diagnose. - Corps fusiforme mesurant jusqu'à 210 p. de long sur 35 p. de large. Extrémité antérieure arrondie mais portant généralement à son sommet un court mucron conique; extrémité postérieure assez longuement acuminée. Surface creusée de sillons longitudinaux profonds souvent disposés en hélice et séparant des côtes arrondies ou des crêtes plus ou moins aiguës qui s'amincissent vers le pôle postérieur et se prolongent souvent en arrière, formant une touffe de filaments qui rappellent ceux de Zygocystis cometa Stein. Parfois ces côtes sont comme crénelées ou ondulées, ces ondulations s'accentuant à tel point que les côtes peuvent se découper en lobules arrondis.

Noyau présentant généralement plusieurs karyosomes. Grains de paramylon assez volumineux. 
Pas de mouvements ; le parasite parait toujours immobile et rigide, comme figé.

Libre dans les vésicules séminales ou retenu par son extrémité postérieure dans les replis des pavillons ciliés.

Stades jeunes dans les cytophores de l'hôte.

Hôte : Lumbricus terrestris L., Müll.

$M$. striata se rencontre dans les vésicules séminales de $L$. terrestris en compagnie des espèces précédentes, mais elle est beaucoup plus rare. Je ne l'ai jamais observée chez les autres Lombriciens que j'ai examinés.

Le parasite se développe dans l'intérieur des blastophores de l'hôte; adulte, il est libre, dans les vésicules séminales ou, le plus souvent fixé, comme nous le verrons, d'une façon particulière par son extrémité postérieure au bord des pavillons ciliés. Sa forme est peu variable. D'abord cordiforme (fig. 32), il rappelle absolument les formes jeunes de Rhynchocystis pilosa, puis la région postérieure s'allonge en s'étirant tandis que la région antérieure s'allonge très peu en s'effilant légèrement. La Grégarine prend ainsi la forme d'un fuseau asymétrique (fig. xxxIv) rappelant $R$. pilosa, mais moins allongé. Je n'ai pas trouvé d'exemplaires dont les dimensions excédassent $210 \mu$ sur $35 \mu$; la longueur moyenne est de 160 p., l'épaisseur moyenne de $30 \mu$.

$M$. striata $\mathrm{m}^{\text {'a }}$ toujours paru complètement immobile, l'ectoplasme épais et très ornementé qui la recouvre lui constitue une enveloppe rigide, sorte d'armure dans laquelle elle est comme figée.

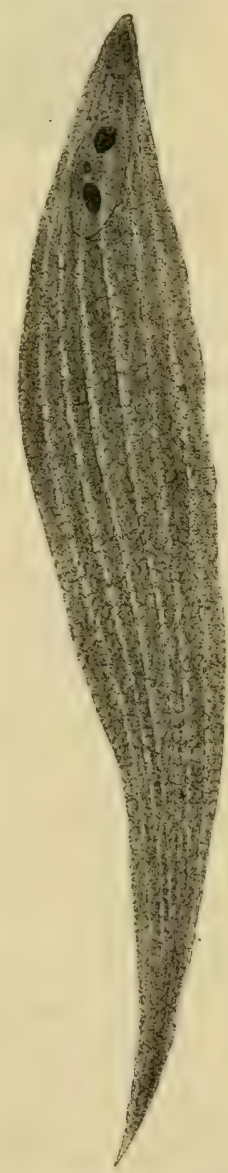

FIG.XXXIV. M. striate adulte fusiforme $\times 500$. 
On observe à son extrémité antérieure un mucron conique constitué par du hyaloplasme dense, absolument comparable

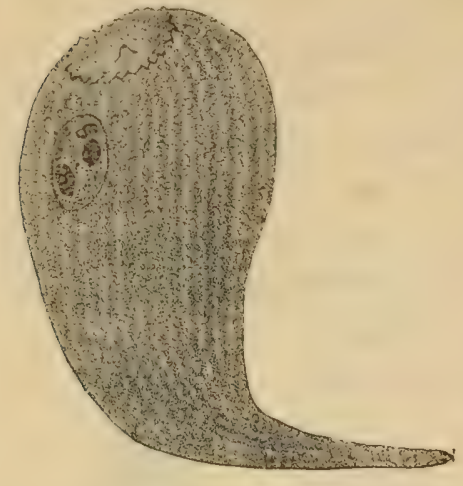

Fig. $\mathrm{XXXV} . \quad M$. striata $\times 750$. Mucron encore enfoncé dans un cratère. Noyau à 3 karyosomes. à celui que j'ai signalé chez $M$. lumbrici, mais un peu plus développé. Ce mucron se teint fortement en vert par le Vert lumière, en violet intense comme les karyosomes par le Kristallviolet (méthode de Benda pour la recherche des Mitochondries). Il n'existe pas à sa surface de formations ectoplasmiques. Dans les parasites peu développés, il. est parfois placé au fond d'un cratère creusé dans l'extrémité antérieure (fig. 32, fig. xxxv), comme cela s'observe aussi chez certaines $R$. pilosa; mais, chez les adultes, il fait toujours saillie à l'extrémité du pôle antérieur.

L'épicyte est aussi mince que chez les autres Monocystis précédemment étudiées, il est orné de stries longitudinales qui sont placées au sommet des côtes dont est ornementée la surface du corps.

La couche de sarcocyte est épaisse et peut atteindre jusqu'à $1 \mu .5$ à $2 \mu$ : Elle est creusée de sillons profonds qui séparent des côtes à forme variable, se présentant sur les coupes transversales comme des lames aplaties, des arêtes triangulaires ou des bourrelets arrondis (fig. xxxvI).

A leur sommet, on aperçoit les stries de l'épicyte. Ces côtes dessinent généralement des lignes méridiennes à la surface du

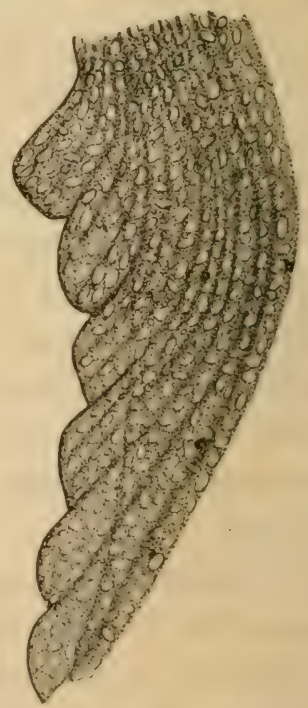

Fig. XXXVI. Côtes do $M$. striala $\times 1.300$. 
corps, mais elles peuvent aussi être disposées en hélice; elles n'ont ni une épaisseur uniforme, ni une même largeur sur toute la longueur du corps du parasite; en avant et en arrière elles sont plus étroites et ne sont formées que par du sarcocyte ; vers le milieu du corps, elles sont plus larges et l'endoplasme peut pénétrer dans leur intérieur avec toutes ses inclusions. Elles se prolongent souvent à l'extrémité postérieure en lanières filiformes, constituant ainsi une sorte de touffe de poils. Parfois leur bord libre est ondulé et le parasite présente alors sur ses bords des lobulations, sortes de bourrelets crénelés, arrondis qui peuvent même prendre l'aspect de sphérules aplaties lorsque le corps est contracté. La figure 96 représente un parasite duquel on voit se détacher quelques-unes de ces boules, ceci est dû à une torsion exagérée du corps de la Grégarine et ce n'est peut-être qu'un artifice de préparation. Il en est sans doute de même des boules que l'on voit pendre à la partie postérieure de Grégarines mutilées et privées de leur queue comme certaines $R$. pilosa.

Je n'ai pas observé les fibrilles du myocyte ; elles sont sans nul doute très peu développées étant donné l'absence presque complète de mouvement chez ce parasite.

L'endoplasme a le même aspect que celui de $R$. pilosa que nous décrivons plus loin, il renferme des grains de paramylon de même forme et des grains chromatoïdes semblables. On observe souvent aussi une masse chromidiale volumineuse à proximité de la queue.

Le noyau est ellipsoïdal ou irrégulier, il est le plus souvent parallèle au grand axe du corps, mais il peut aussi quelquefois se placer perpendiculairement à cet axe ; médian dans les formes jeunes, il est presque toujours placé près de l'extrémité antérieure, au voisinage du mucron, chez l'adulte.

Il renferme un karyosome volumineux qui se fragmente le plus souvent en 2, 3 ou 4 karyosomes plus petits (fig. $\mathrm{xxxv).} \mathrm{Le} \mathrm{reste}$ du noyau est rempli par un coagulum granuleux sans affinité pour les colorants de la chromatine. Je n'ai pas vu de réseau de linine. 
Les karyosomes sont vacuolaires, ils présentent l'aspect et les affinités colorantes que nous retrouverons pour celui de $R$. pilosa. Ils donnent par bourgeonnement des grains chromatiques qui se dispersent dans le noyau et peuvent même passer directement dans le cytoplasme. On observe ici en effet des " noyaux flammés " analogues à ceux que nous avons décrits chez $M$. agilis.

Comme dans $R$. pilosa, le mucron doit être le siège d'échanges nutritifs intenses, car le hyaloplasme mucronaire est souvent rempli de nombreuses granulations chromatoïdes. Dans ce cas, le noyau est tangent à la surface du hyaloplasme de la trompe, ou même, il baigne partiellement dans l'intérieur de ce hyaloplasme. Sa structure est normale.

Le développement a lieu dans les blastophores de l'hôte, et il est absolument semblable à celui de $R$. pilosa, seulement, il ne se forme pas de poils et, l'épimérite étant peu développé, le parasite ne reste pas suspendu au blastophore nourricier, il s'en détache dès qu'il est sorti de l'intérieur.

Les $M$. striata adultes sont libres dans les vésicules séminales, on les trouve surtout rassemblées en nombre considérable sur les bords libres de l'entonnoir cilié des pavillons séminaux; elles occupent ainsi la situation que nous retrouverons dans les formes C de $R$. pilosa; leur mode de stabulation est aussi très comparable, leur extrémité postérieure est étroitement appliquée entre les cellules saillantes qui bordent ces pavillons et les cils s'engagent dans les sillons hélicoïdaux de la queue du parasite maintenant celui-ci immobile au milieu des spermatozoïdes de l'hôte dont le mode de fixation est à peu près le même. Lorsque la Grégarine est pourvue d'une touffe de poils terminale, ces poils s'insinuent entre les cils, ainsi que nous le verrons aussi pour $R$. pilosa $\mathrm{C}$, et assurent d'une façon plus complète encore le maintien du parasite. Ces singuliers modes de fixation par l'extrémité postérieure, nous montrent combien il faut être circonspect lorsqu'on veut se baser sur la fixation pour orienter le corps d'un parasite. 
De même que les espèces précédemment étudiées, $M$. striatu est attaquée parfois par une Bactérie spéciale qui provoque la dégénérescence de la Grégarine.

\section{Monocystis hirsuta n. sp.}

J'ai observé dans les vésicules séminales de Lumbricus castaneus Sav. une curieuse Grégarine, remarquable par sa toison formée de poils épais et larges, souvent enchevêtrés et qui lui vaut son nom.

Diagnose. - Monocystidée à corps ellipsoïdal mesurant jusqu'à 225 is sur 90 p. Pôle antérieur présentant un mucron formé de cytoplasme très dense et souvent très développé. Corps recouvert de poils larges et épais, souvent en broussaille. Stries épicytaires longitudinales fines et serrées. Noyau à karyosome unique très volumineux.

Hôte : Lumbricus castaneus Sav.

Ce parasite est très rare, je ne l'ai rencontré que dans quelques exemplaires de Lumbricus castaneus Sav. recueillis à Montessaux; le même hôte capturé aux environs de Grenoble ne m'a jamais paru envahi par la même Grégarine. Celle-ci habite les vésicules séminales et paraît rester toujours extracellulaire.

Les formes observées étaient sub-sphériques, ovoïdes ou ellipsoïdales (fig. xxxvII), quelques-unes atteignaient jusqu'à $225 \mu$ sur $90 \mu$. Elles ne présentent que des mouvements très lents, à peine sensibles. A la partie antérieure du corps qui est généralement un peu renflée, on aperçoit un mucron conique semblable à celui que nous avons signalé déjà chez d'autres Monocystis, c'est-à-dire formé d'un cytoplasme très dense, non granuleux ou formé de granulations excessivement fines et serrées. Ce mucron est très développé, il rappelle par son volume la trompe que nous décrirons chez $R$. pilosa, mais il est toujours nu et dépourvu de la couronne de sarcocyte, caracté- 
ristique de cette dernière espèce (fig. xxxvII, xxxviII, 33).

'L'épicyte présente des stries longitudinales fines et serrées. Au-dessous existe une couche épaisse de sarcocyte. Je n'ai pas aperçu le myocyte peu développé.

La surface du parasite est recouverte d'une toison dense de poils larges, coniques ou irréguliers, très allongés, constitués

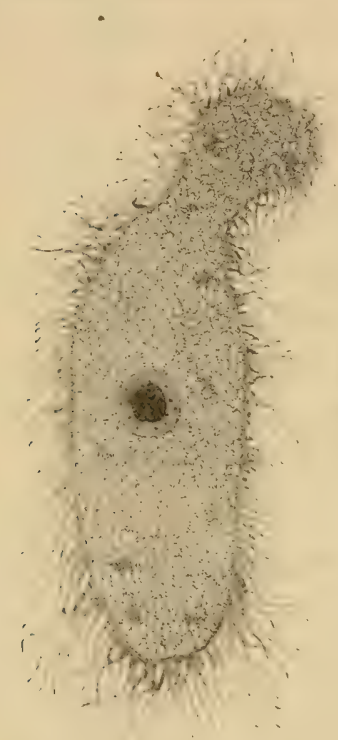

$X X X V I I$

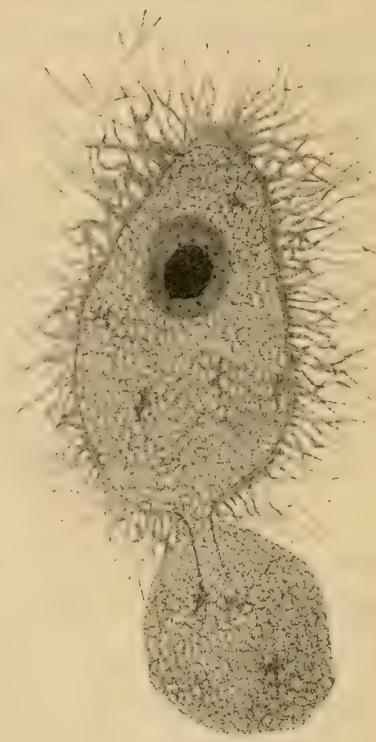

XXXVIII.

FIG. XXXVII. $\boldsymbol{M}$. hirsuta $\times 300 .-$ FIG. XXXVIII. $\boldsymbol{M}$, hirsuta $\times 300$. Cuticule rompuo postérieurement.

par le sarcocyte dense recouvert par l'épicyte et dans lesquels pénètrent parfois les granulations entocytiques et même les grains de paramylon. Ces poils sont serrés et irrégulièrement disposés, souvent enchevêtrés les uns dans les autres ; ceux qui recouvrent le pôle postérieur sont rassemblés en une touffe épaisse dirigée en arrière ; ils sont généralement plus longs que les autres, et, chez les jeunes parasites, ils atteignent et souvent même dépassent la longueur du corps (fig. 33). Dans les parasites adultes, cette longueur est moins considérable et 
la forme des poils est très variable, ce sont de longues lanières coniques ou de petites boules pédiculées supportant un long filament délié (fig. 34) ou encore des lames coniques rappelant les bourrelets latéraux de $M$. striata. Cette toison n'est pas très résistante, surtout vers la région postérieure du corps, elle se déchire souvent en ce point et l'on peut voir sur des frottis le corps de la Grégarine faire hernie hors de son enveloppe hérissée sans que les granulations entocytiques s'écoulent au dehors, et prendre la forme d'une boule rattachée au reste du parasite par un court pédicule parfois très mince (fig. xxxvIII).

L'endoplasme est formé de fines granulations très denses, parmi lesquelles on observe, chez les jeunes stades de nombreux grains sidérophiles qui disparaissent lors de l'apparition du paramylon. Celui-ci est en grains ellipsoïdaux assez volumineux et fortement tassés.

Le noyau sphérique ou ellipsoïdal occupe une situation variable; il renferme un volumineux karyosome vacuolaire dont le diamètre atteint presque la moitié de celui du noyau et autour duquel irradient les fins filaments de linine. Il n'y a pas, le plus souvent, de grains chromatiques dans le reste du noyau; s'il en existe, ils sont disposés en cercle autour du karyosome.

Les plus petites formes observées, mesurant $18 \mu$ sur $16 \mu$ étaient déjà extra-cellulaires, et présentaient le revêtement de longs poils larges caractéristiques de l'espèce. Le noyau est très volumineux et il renferme un énorme karyosome dont le diamètre est supérieur à la moitié du diamètre propre du noyau. La présence de ce noyau très développé permet de distinguer facilement les jeunes $M$. hirsuta des jeunes $R$. pilosa qui sont du reste à cette taille encore contenues dans les blastophores de l'hôte.

Monocystis crenulata $\mathrm{n}$. sp.

Monocystis crenulata est une Grégarine sub-sphérique, ovoïde ou réniforme atteignant jusqu'à $120 \mu$ sur $105^{\circ} \mu_{\text {_ et }}$ paraissant 
complètement immobile. Région antérieure aplatie en un plateau entouré de dents. Ectoplasme recouvert de villosités irrégulières. Entocyte à aspect très sombre in-vivo. Noyau à plusieurs karyosomes

Hôtes : Helodrilus longus Ude. et $H$. caliginosus var. trapezoïdes Ant. Dugés.

Cette Monocystis est spéciale aux $H$. longus et $H$. caliginosus var. trapezoïdes ; je ne l'ai pas observée chez $H$. caliginosus type. Le parasite ne se rencontre pas souvent, mais il est assez abondant chez les individus infestés. Il habite les vésicules séminales.

Sa forme est variable, tantôt presque sphérique ou ovoïde, elle devient parfois réniforme ou cordiforme. Les plus gros exemplaires examinés mesuraient $120 \mu$ sur $105 \mu$. Nous n'avons jamais vu le parasite en mouvement. In-vivo, il a un aspect très sombre dû à la structure particulière de son entocyte et il apparaît bordé par une enveloppe épaisse d'ectoplasme hyalin.

La région antérieure est souvent déprimée en un plateau circulaire bordé de denticulations larges et assez élevées et présentant en quelque sorte l'aspect du sommet d'une tour bordée de créneaux (fig. xxxIx). Quelquefois c'est la région postérieure qui se déprime et présente une cavité peu profonde ; d'autres fois au contraire elle se prolonge en une courte pointe mousse paraissant uniquement constituée de sarcocyte (fig. $\mathrm{xxxIx}$ ).

L'épicyte est mince, il recouvre une couche de sarcocyte très épaisse ; je n'ai pas vu de myocyte.

Les denticulations qui bordent le plateau antérieur sont constituées par du sarcocyte, mais elles peuvent contenir aussi de l'endoplasme granuleux et des grains de paramylon (fig. 36). Elles sont parfois très développées et peuvent atteindre 10 à $11 \mu$ de large sur 7 à $8 \mu$ de hauteur ; elles ne sont pas toujours régulières, mais elles peuvent se lacinier, s'allonger en filaments 
irréguliers plus ou moins renflés; il s'en détache quelquefois de petites boules comme si elles étaient en dégénérescence (fig. 38).

Sur le reste du corps, l'ectoplasme n'est pas lisse, il se montre hérissé de villosités courtes, irrégulières, parfois laciniées et formées de sareocyte comme les dents du créneau, mais dans lesquelles ne pénétrent jamais les granulations de l'entocyte (fig. 37).

L'entocyte est formée de grossières granulations opaques in-vivo, ce qui donne au parasite l'aspect sombre signalé plus haut. Parmi ces granulations s'observent quelques sphères chromidiales assez volumineuses. Des alvéoles irrégulières, peu serrées sont creusées dans ce cytoplasme; elles renferment les grains de paramylon sphériques ou en bâtonnets allongés et peu volumineux.

Le noyau sphérique renferme constamment

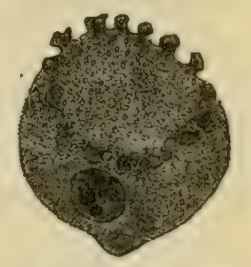

FIG. XXXIX. M. crenu lata $\times 300$. plusieurs karyosomes, quatre ou davantage, dont quelques-uns sont accolés à la membrane nucléaire. Ces karyosomes sont, les uns sphériques, avec de petits grains chromatiques appliqués à leur surface, les autres tout à fait irréguliers, mamelonnés, semblant s'émietter en particules plus petites qui se dispersent dans le noyau. Tous sont vacuolaires (fig. 39).

Nous n'avons trouvé aucun stade jeune de ce parasite et nous ignorons complètement son développement.

Les kystes et sporocystes de $M$. crenulata ont la même structure que celle des kystes et sporocystes des autres Monocystis habitant avec elle les vésicules séminales des Helodrilus et il n'est pas possible de les en distinguer.

Monocystis Le Mêmei n. sp.

Dans quelques exemplaires de Helodrilus caliginosus Sav. provenant d'El-Kantara (Algérie), j'ai trouvé une Monocystidée à laquelle je donnerai lo nom de Monocystis Le Mêmei, la dédiant 
à mon ami le Dr Le Même qui a eu l'amabilité de me procurer les hôtes. Les mêmes Oligochètes recueillis en France ne renferment jamais ce parasite, ils hébergent, outre $H$. crenulata, deux espèces décrites plus loin : $N$. lumbricoïdes et $P$. Cuénoti.

Diagnose. - Monocystidée ovoüde ou ellipsoïdale mesurant en moyenne $200 \mu$ sur $120 \mu$; mais pouvant atteindre dans le colome $650 \mu \mathrm{sur}$ 300. Ectoplasme très mince. Endoplasme alvéolaire renfermant des grains de paramylon volumineux, sphériques; aux noeuds du réseau cytoplasmique, amas de grains volumineux renfermés dans suc coloré en bleu parle Mann. Noyau central ou plus proche de l'une des extrémités. Karyosome formé de deux parties, l'une massive, l'autre constituée par une agglomération de grains chromatiques. Sporocystes semblables à ceux des autres Monocystis des Lombriciens.

Hôte : Helodrilus caliginosus Sav. provenant d'El-Kantara.

Monocystis Le Mêmei était très abondante dans les vésicules séminales des Helodrilus caliginosus Sav. recueillis durant le mois d'avril à El-Kantara; d'autres exemplaires du même hôte provenant de la même localité, examinés en juillet renfermaient des kystes en abondance, mais moins de stades végétatifs. Des parasites et des kystes se rencontrent aussi dans le cœlome.

Le parasite est ovoïde ou ellipsoïdal, parfois presque sphérique, il peut atteindre jusqu'à $200 \mu$ sur $150 \mu$. Nous ne l'avons jamais vu en mouvement; il est du reste souvent entouré par les phagocytes de l'hôte.

En dehors de la forme de l'un des pôles, en général plus pointu que l'autre, il n'existe pas de différenciation qui permette de distinguer une extrémité antérieure ou postérieure, il n'y a pas trace de mucron (fig. $\mathrm{xL}$ ).

L'épicyte, très mince, ne montre pas d'ornementation. La couche de sarcocyte est le plus souvent à peine perceptible et nous n'avons pas réussi à mettre en évidence le myocyte sạns doute rudimentaire, 
L'entocyte est creusé de larges alvéoles contenant des grains de paramylon sphériques assez volumineux. Les mailles de spongioplasme renferment de fines granulations colorées les unes en bleu, les autres en rouge par le Mann. Aux nœuds du réseau, on observe un suc coloré en bleu par le Mann, en gris par le Fer et dans lequel se rencontrent fréquemment des amas de grains bleus plus volumineux que cenx du spongioplasme granuleux. C'est aussi en ces points que, chez les parasites phagocytés en voie de dégénérescence, ou chez ceux qui sont en train d'absorber leurs réserves, on observe des gouttelettes colorées en rouge par le Mann et analogues à celles que l'on retrouve chez d'autres espèces parasites des Oligochètes.

Le noyau est généralement placé au centre, cependant il peut aussi quelquefois se rapprocher de l'une ou l'autre des extrémités, tout en restant dans l'axe du parasite. Il est entouré d'une membrane chromatique et renferme un karyosome assez volumineux et un réseau de linine sur lequel sont dispersés des grains de chromatine, colorés en vio-

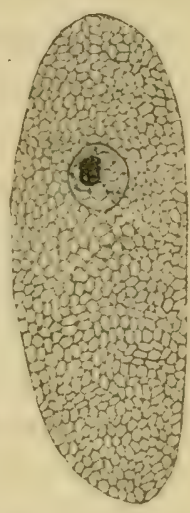

FIG. $\mathrm{XL}, M, L e$ Iêmei $\times 300$. let ou bleu violacé par le Mann et des grains de pyrénine, colorés en rouge par le Mann.

Ce karyosome est complexe; en général, il se compose de deux parties : une masse sphérique vacuolaire qui se colore comme la pyrénine, et, tangent en un point de la surface de cette sphère, un amas granuleux généralement coloré comme la chromatine. Cet aspect du karyosome rappelle dans une certaine mesure celui que LÉGER et DuBoscQ (1903) ont décrit chez Adelea dimidiata coccidioüdes des Scolopendres de Corse ; mais les deux parties du karyosome sont accolées l'une à l'autre chez Monocystis Le Mêmei tandis que chez la Coccidie, elles sont séparées par une large zone claire. LÉGer et DuboscQ supposent que le karyosome granuleux est émané de l'autre par 
bourgeonnement. C'est aussi ce qui semble ressortir de l'examen de nos préparations, en ce qui concerne la Grégarine.

Le karyosome granuleux manque quelquefois; quand il existe, il est toujours tangent au karyosome sphérique dont la présence est constante, ou relié à lui par un large pédicule généralement très court. Le karyosome sphérique présente la structure normale du karyosome des Monocystis; c'est une masse vacuolaire recouverte par une couche plus compacte très mince. Le Mann colore en rouge la masse centrale, en violet la croûte externe; celle-ci est soulevée et semble même rompue en certains points par de gros grains rouges émanés de l'intérieur et un peu plus pâles que la masse centrale. Autour de l'un de ces grains, on voit s'étaler le karyosome granuleux ; il donne l'impression d'une masse échappée du karyosome sphérique par une sorte de goulot et qui se serait étalée autour de ce goulot, à la surface de la sphère dont elle est issue, en une calotte plus ou moins étendue (fig. 184).

On constate souvent dans eet ensemble des variations de coloration; ainsi, quelquefois le karyosome sphérique seul présent est formé par une moelle vacuolaire entourée d'une épaisse croûte colorée en rouge par le Mann, la moelle étant colorée en violet. Parfois le karyosome sphérique présente cette même structure et l'on voit accolé à lui le karyosome granuleux complètement coloré en violet. D'autres fois le karyosome granuleux, au lieu d'être formé de grains uniformément colorés en violet, se compose de deux parties : sur la surface de la calotte qui le constitue on observe une série de grains rouges de diverses grosseurs partant d'un grain rouge bourgeonné par le karyosome sphérique, au-dessous de ces grains on en observe d'autres plus petits, virant au violet, enfin le reste de la masse granuleuse est formée de grains très fins, violet foncé. Les figures 184 et 185 montrent deux aspects tout différents de ce karyosome. Ces faits montrent qu'il y a des relations très étroites entre la plastine et la chromatine et ils viennent à l'appui des vues de Monoff (1907) qui considère plastine et chromatine comme 
deux états très voisins d'une même substance, tantôt basophile, tantôt acidophile.

Le réseau de linine est parfois masqué par un coagulum granuleux que le Mann colore en gris ; les filaments de ce réseau sont colorés en bleu d'azur par le Mann, ils supportent de fines granulations colorées en violet (chromatine), ou en rouge (plastine, pyrénine); ces deux sortes de granulations coexistent assez fréquemment.

Les kystes de $M$. Le Mêmei sont sphériques ou ovoïdes et renferment des sporocystes qui ne diffèrent de ceux des autres Monocystis ni par la forme, ni par la structure; ces sporocystes mesurent $9 \mu$ sur $4 \mu .5$.

Je n'ai pas trouvé de stades très jeunes du parasite; les plus petits exemplaires observés étaient déjà extra-cellulaires, peut-être n'y a-t-il pas de stade intrablastophorien. Ces jeunes Grégarines sont ovoïdes, leur cytoplasme est dense, finement granuleux dépourvu de grains de réserve. Le noyau ne contient que le karyosome sphérique. A un certain moment, les grains rouges de petite taille sont émis par le noyau dans le cytoplasme, ils y restent visibles jusqu'à l'apparition des grains de paramylon, et disparaissent peu à peu à mesure que se forment ces grains de réserve.

Lorsque le parasite arrive au terme de sa croissance, il y a une nouvelle émission de grains rouges dans le cytoplasme ; ces grains sont plus volumineux que les grains émis précédemment, leur sortie suit la formation d'un " noyau flammé " de la même façon que nous avons relatée déjà chez $M$.agilis. Ces grains jouent sans doute le rôle de ferments destinés à faciliter l'absorption des grains de réserve qui commencent à disparaître à partir de l'arrivée des grains rouges dans le cytoplasme.

Le parasite peut accomplir toute son évolution dans les vésicules séminales; mais il peut aussi passer dans le cœlome Là, il grossit considérablement, sans changer de forme, et peut atteindre jusqu'à $650 \mu$ de long sur 300 d'épaisseur. Son noyau, d'une soixantaine de $\mu$. de diamètre, renferme un karyosome 
de $25 \%$ de diamètre, en moyenne. La structure reste la même que celle des individus contenus dans les vésicules séminales.

En général, les parasites cœlomiques sont entourés par la membrane péritonéale qui s'applique si étroitement contre leur cuticule qu'elle semble faire partie intégrante du parasite. Celui-ci semble ainsi posséder une sorte d'épiderme pluricellulaire.

M. Le Mêmei est souvent attaquée par les phagocytes; le parasite ne résiste pas à ces attaques et il dégénère, comme nous le verrons plus loin.

Monocystis Elmassiani n. sp.

J'ai observé chez Dendrobona rubida, var. subrubicunda une Monocystis nouvelle que je dédie à M. le Dr Elmassian, en voici la diagnose.

Monocystis très allongée, peu épaisse, de $750 \mu$ sur $25 \mu$. Extrémité antérieure renflée, souvent très fortement en boule, extrémité postérieure pointue. Ectoplasme assez épais; stries longitudinales fines et serrées, souvent en spirales. Endoplasme finement granuleux. Grains de paramylon sphériques ou en bâtonnets, de taille fort variable. Noyau ovoüde très grand renfermant en général de nombreux karyosomes.

Hôtes : Dendrobona rubida, var. subrubicunda Eisen, Helodrilus longus Ude.

Ce parasite est assez fréquent dans les vésicules séminales de Dendrobona rubida, var. subrubicunda Eisen; je l'ai rencontré en outre une fois dans le cœlome d'un Helodrilus longus Ude. Sa forme rappelle un peu celle de $R$. porrecta, mais la région antérieure est beaucoup plus fortement renflée que chez cette dernière espèce, elle ne contient pas le noyau et en outre ce noyau a une structure toute différente. Le corps peut atteindre $25 \mu$ de large, pour une longueur de $750 \mu$; en arrière de la 
tête, il présente une partie plus étroite parfois assez allongée (fig. XLI).

L'extrémité antérieure est toujours fortement renflée en boule rappelant celle de ccrtains $R$. porrecta, ou plutôt, beancoup plus exactement celle du Selenidium " en point et virgule » de CAULLERY et MESNIL (1899). Cette région se colore souvent bien plus fortement que le reste du corps, elle est comparable au mucron que nous avons signalé précédemment chez un grand nombre d'espèces (fig. 35).

L'extrémité postérieure se termine en pointe.

L'ectoplasme est toujours assez épais ; il présente de fines striations longitudinales ser-
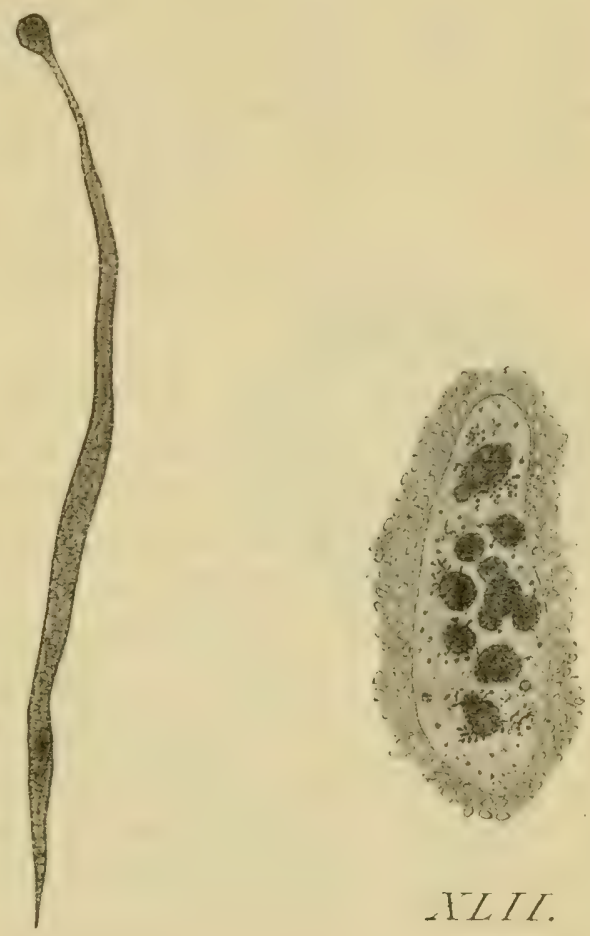

ILI.

FIG. XII. MI. Elmassiani $\times 120 .-$ HIG. XLII. Noyau de 11. Elmassiani $\times 1.300$.

rées, souvent en

spirale dans la région postérieure du corps et qu'il faut très probablement considérer comme des fibrilles du myocyte.

L'endoplasme finement granuleux est creusé d'alvéoles qui renferment comme d'habitude les grains de paramylon. Ceuxci sont très variables comme forme et comme dimensions; parfois ils sont peu serrés, sphériques ou ovoïdes; d'autres fois, 
au contraire, très fortement tassés, ils se présentent comme de petits bâtonnets allongés.

Le noyau est très grand et ovoïde; il renferme toujours plusicurs karyosomes, souvent jusqu'à 10, qui paraissent provonir par émiettement les uns des autres. J'ai observé parfois des séries de petits grains sphériques à disposition arborescente qui paraissaient provenir par bourgeonnement d'un des karyosomes les plus volumineux (fig. XLII).

Je n'ai pas suivi le développement de cette espèce.

\section{Monocystis tumbrici olidi Schmidt.}

Scmmot (1854) a observé chez Lumbricus olidus Hoffm., une Grégarine arrondie, couverte de poils qu'il représente 1). XiV, fig. 16 ; Lieberkühn (1855) représente aussi des Monocystis arrondies ou ovales couvertes de poils (pl. VII, fig. 9, 10, 15). J'ai retrouvé également ces parasites qui sont assez fréquents chez Dendrobona rubida Sav.

Dragnose. - Monocystidée arrondie, ovoïde ou réniforme de $90 \mu$ à $100 \mu$ de diamètre; couverte de poils courts et serrés tantôt fins, tantôt assez épais. Sarcocyte mince. Entocyte renfermant des grains de paramylon peu volumineux, à peu près sphériques, de taille inégale. Noyau sphérique à deux karyosomes l'un irrégutier, vacuolaire, peu colorable par le Fer, l'autre arrondi, dense, fortement sidérophile.

Hôtes : 'Dendrobcena rubida Sav.et sa variété D. sub-rubicunda Eisen, Eisenia foetida Sav. (=L. olidus Hoffm.).

J'ai rencontré celte espèce assez couramment chez Dendrobona rubida Sav. et sa variété sub-rubicunda Eisen et aussi chez Eisenia foetida Sav., où elle est plus rare.

Elle est sphérique, ovoïde ou réniforme mesurant en moyenne $80 \mu$ sur $100 \mu$, ou 90 à $100 \mu$ de diamètre.

Elle paraît le plus souvent immobile; mais elle peut aussi présenter des mouvements assez vifs et qui rappellent d'une 
façon saisissante ceux de l'abdomen d'une almée agitée par la danse du ventre.

La cuticule est mince, sans stries ornementales visibles. Souvent, toute la surface du corps est recouverte de poils toujours assez courts et paraissant plus serrés dans la région postérieure. Parfois, on n'en observe que sur une calotte polaire où ils forment un revêtement épais, ils peuvent alors en cette région prendre la forme de baguettes courtes et assez épaisses (fig. XLIII).

L'endoplasme est creusé d'alvéoles où sont, comme toujours, logés les grains de paramylon. Ceux-ci sont de taille très inégale, mais toujours peu volumineux, ils sont sensiblement sphériques. Le réticulum spongioplasinique renferme des grains dont les uns se colorent en rouge et les autres en bleu par le Mann.

Le noyau contient presque tou-

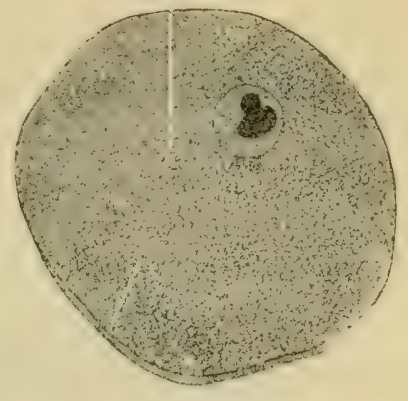
jours deux karyosomes. L'un deux, Fig. XLIII. M. lumbrici olidi $\times 350$. irrégulier et vacuolaire est généra-

lement coloré par le Mann en violet, pariois en rouge violacé avec une calotte violette. L'autre est coloré en rouge par la même méthode, il est régulièrement sphérique et contient une vacuole centrale au milieu de laquelle il y a un grain rouge; il est beaucoup moins volumineux que le précédent (fig. 183). Ces deux karyosomes sont parfois accolés l'un à l'autre, parfois plus ou moins éloignés et même situés aux deux extrémités d'un même diamètre du noyau. Le karyosome sphérique se colore fortement par le Fer, tandis que l'autre est peu colorable.

Il peut n'exister qu'un seul karyosome qui est alors sphérique, vacuolaire et se colore en rouge violacé par le Mann.

Le réseau de linine bien apparent supporte des grains chromatiques très fins disséminés sur toute sa surface.

Je ne connais rien du développement de ce parasite. 


\section{Monocystis villosa $\mathrm{n}$. $\mathrm{sp}$.}

Une autre Grégarine arrondie et velue, voisine de $M$. lumbrici olidi Schmidt, mais s'en éloignant par quelques caractères se rencontre chez Octolasium lacteum Örley, nous l'appellerons M. villosa.

Diagnose. - Monocystidée arrondie, de 200 à $250 \mu$ de diamètre, couverte de poils fins, serrés, souvent très longs. Sarcocyte mince. Entocyle à grains de paramylon volumineux. Noyau sphérique à 1 seul karyosome.

\section{Hôte : Octolasium lacteum Örley.}

Cette Grégarine est assez commune dans les vésicules séminales de Octolasium lacteum Örley, où on la rencontre dans le voisinage de $M$. herculea

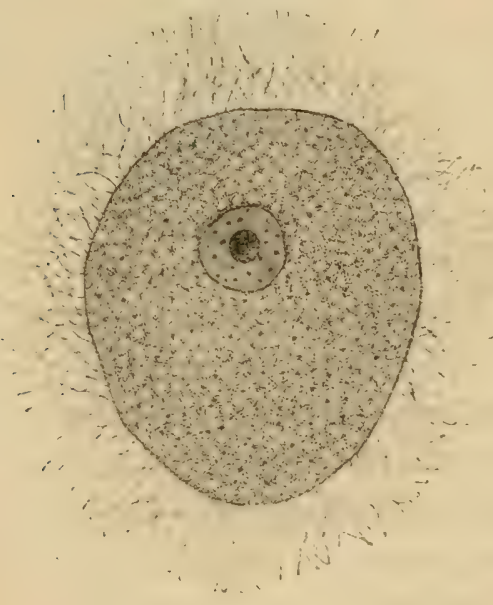

FIG. XLIV. $M$. villosa $\times 200$. et de $M$. turbo que nous décrivons plus loin.

f Elle rappelle par sa forme $M$. lumbrici olidi, mais elle est plus régulièrement sphérique, rarement un peu ovoïde ; elle mesure jusqu'à $250 \mu$ de diamètre. Les poils fins et serrés qui recouvrent la surface du corps sont plus allongés que chez $M$. lumbrici olidi (fig. XLIV); ils sont parfois très longs, surtout dans les formes jeunes, leur longueur dépassant le diamètre du corps.

Le parasite est presque toujours immobile, cependant il peut aussi présenter des mouvements de cyclose tout à fait semblables à ceux que nous venons de décrire chez $M$. lumbrici olidi. 
L'épicyte, mince, ne paraît pas présenter de stries méridiennes. Nous venons de voir que, chez les parasites jeunes, il porte de longs poils, fins et très serrés. Cette toison oppose une grande résistance à la pénétration des réactifs et il est fort difficile d'avoir des Grégarines fixées d'une façon convenable. La cuticule est le plus souvent détachée du reste du corps et celui-ci paraît rempli dans la région périphérique de volumineuses vacuoles. Dans les Grégarines âgées, volumineuses, les poils sont en général moins serrés, plus courts, plus épais ; ils peuvent même disparaître totalement. C'est un phénomène que nous retrouverons chez $R$. pilosa.

La couche de sarcocyte hyalin, dépourvu de granulations est toujours très peu développée; nous n'avons pas observé le myocyte.

L'endoplasme finement granuleux est très dense chez les formes jeunes; chez les adultes, il se creuse de larges alvéoles dans lesquelles sont contenus de volumineux grains de paramylon. Beaucoup d'exemplaires montrent une zone d'endoplasme périphérique claire, creusée de grandes vacuoles, mais cette différenciation, qui s'observe principalement chez les parasites où il y a des décollements de la cuticule, est due sans doute, ainsi que nous l'avons dit plus haut à une insuffisance de fixation.

Dans les jeunes stades, avant le développement des grains de réserve, le cytoplasme est souvent rempli de fines granulations chromatoïdes qui disparaissent après la formation du paramylon.

Le noyau sphérique renferme en son centre un karyosome également sphérique; il est parcouru par un réseau de linine sur lequel sont disposés des grains de chromatine assez développés ; mais le plus souvent toute la chromatine semble condensée dans le karyosome. Celui-ci dans les préparations colorées par l'Hémalun se montre entouré par une sorte de croûte fortement colorable; on observe en son centre une masse médullaire à contour plus ou moins régulier et qui présente un aspect 
spumeux comparable à celui que dessine Siedtecki (1905) chez Caryotropha Mesnili (pl. XVI, fig. 5); tout autour s'étend une zone moins colorée et dans laquelle se trouvent quelques petites vacuoles sphériques. Cette structure vacuolaire n'est pas mise en évidence par le Fer. Le karyosome est toujours unique; il semble bourgeonner des grains chromatiques de diverses tailles que l'on peut voir accolés à sa surface ou placés à une eertaine distance et rattachés à cette surface par des filaments très déliés (fig. XLV.)

Nous n'avons jamais observé de stades intra-cellulaires.

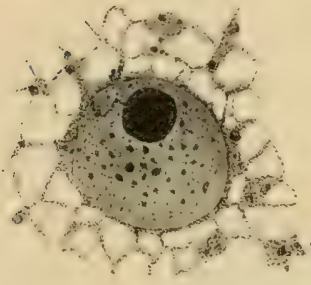

FIG. XLV. Noyau de $\boldsymbol{M}$. villosa $\times 1300$.

Le parasite est quelquefois attaqué par des Bactéries, de même que les diverses espèces de Monocystis des Lombriciens; il est parfois aussi englobé par les phagocytes de l'hôte et absorbé par ceux-ci après s'être hypertrophié assez fortement.

J'ai rencontré dans les vésicules séminales d'Octolasium des kystes de $120 \mu$ à $200 \mu$ de diamètre et qui appartiennent à $M$. villosa ou à $M$. turbo, les deux plus petites Grégarines que j'ai observées chez cet hôte. Ils renferment des sporocystes ayant la même forme et la même structure que ceux des espèces décrites chez les Lombriciens mais dont les dimensions varient de 13 u. 5 à $22 \mu 5$ de long sur $7 \mu$ à $10 \mu 5$ de large.

\section{Monocystis turbo n. sp.}

On rencontre presque constamment dans les vésicules séminales d'Octolasium lacteum Örley des environs de Grenoble une Grégarine qui ne paraît pas avoir été décrite, et que nous nommons $M$. turbo en raison de sa forme la plus habituelle en toupie.

Diagnose. - Monocystidée de petite taille ne dépassant pas $80 \mu$ de diamètre. Forme en cour ou en toupie. Mouvements, en 
sablier. Ectoplasme mince sans ornementation visible. Enloplasme grossièrement granuleux contenant des grains de puramylon sphériques, volumineux, peu serrés. Noyau sphérique renfermant un gros karyosome également sphérique.

\section{Hôte : Octolasium lacteum Örley.}

M. turbo est très fréquente dans les vésicules séminales de Octolasium lacteum Örley, je ne l'ai pas observée chez d'autres hôtes.

Sa forme est celle d'un cœur ou d'une toupie; exceptionnellement, elle peut être sphérique ; c'est la plus petite des Grégarines parasites des glandes séminales que nous ayons étudiées; elle ne mesure pas plus de $75 \mu$ de diamètre ou $80 \mu$ sur $70 \mu$. Ses mouvements rappellent ceux de $1 /$. lumbrici, mais ils sont excessivement lents. Parfois au lieu d'être animées de ce mouvement en sablier, les granulations cytoplasmiques et les grains de réserve tournent lentement en cercle, à peu près de la même façon que chez $M$. villosa, mais sans que la forme extérieure du parasite soit affectée d'une façon appréciable par ce mouvement.

Il n'existe rien ici de comparable à l'épimérite ou au mucron des espèces décrites précédemment.

L'ectoplasme est très réduit, il comprend un mince épicyte sur lequel nous n'avons pas réussi à apercevoir de stries d'ornementation et une faible couche de sarcocyte. Nous n'avons pas vu les fibrilles du myocyte.

L'endoplasme est creusé de larges alvéoles renfermant des grains de paramylon volumineux, le plus souvent sphériques et atteignant jusqu'à $8 \mu$ de diamètre ; les travées cytoplasmiques sont larges, constituées par de grosses granulations, elles renferment quelques grains sidérophiles; aux nœuds du réseau cytoplasmique on aperçoit de volumineuses sphérules chromatoïdes.

Le noyau sphérique occupe le centre du corps, mais il peut se déplacer pendant les mouvements du parasite de même 
que les granulations cytoplasmiques. Il renferme un gros karyosome également sphérique, central ou légèrement excentrique, creusé de vacuoles et présentant à sa surface uno croûte plus colorée. Ce karyosome est entouré d'une zone circulairo claire produite peut-être par l'action des agents fixateurs et dans laquelle on aperçoit un grand nombre de petits filaments rayonnants paraissant s'attacher au karyosome et divergeant dans le noyau (fig. xLvr). Ce sont les filaments du réseau de linine sur lesquels sont disposés quelques grains chromatiques.
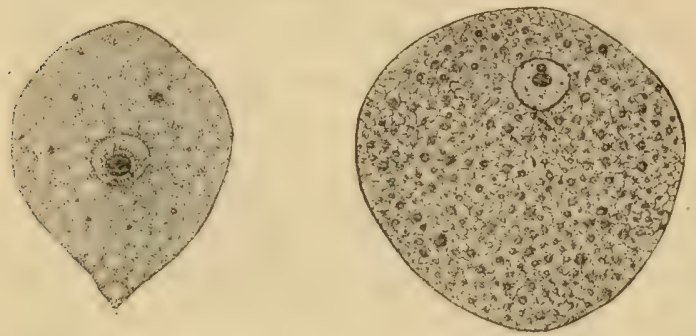

Il y a souvent un ou deux gros grains chromatiques accolés au karyosome et qui proviennent probablement de ce karyosome FIG. XLVI. MI. Turbo $\times 300,-$ FIG. XLVII. MI. herculea $\times 60$. par bourgeonnement.

Le développement a lieu dans les blastophores de l'hôte que le parasite quitte lorsque son accroissement est terminé.

Nous avons dit en parlant de $M$. villosa, qu'il n'était pas possible de distinguer les kystes et sporocystes des deux Grégarines qui cohabitent dans les vésicules séminales du même hôte.

Monocystis herculea Bosanquet.

Bosanquet (1894) a décrit dans le cœlome de Lumbricus herculeus Sav. une Grégarine arrondie très volumineuse qui avait été vue auparavant et figurée par LIEBERKÜHN (1855, fig. 4,6 , pl. VIII). Nous avons retrouvé le même parasite dont nous donnons la diagnose :

Monocystis sphérique ou ovoïde, de grande taille, atteignant jusqu'à 3 à 4 mm. de diamètre. Couleur d'un blanc de lait. Epicyte orné de stries méridiennes fines et serrées. Sarcocyte épais. Fibrilles du myocyte à peine perceptibles. Noyau sphérique, 
renfermant en général plusieurs karyosomes sphériques et quelques grains chromatiques sur un fin réseau de linine. Grains de paramylon sphériques, atteignant jusqu'à $15 \mu$ de diamètre. Kystes très volumineux et renfermant les uns des sporocystes de $30 \mu$ sur $12 \mu$, les autres des sporocystes de $15 \mu$ sur $5 \mu$.

Hôtes : L. herculeus, Octolasium lacteum.

Habitat et sik̀ge. - M. herculea, se rencontre dans lo cclome de L. terrestris, L., M., principalement dans le voisinage de l'extrémité postérieure, et chez Octolasium lacteum Örley, dans les vésicules séminales et dans le cœlome. Le parasite n'est pas très commun chez L. terrestris ; je l'ai observé surtout dans des individus jeunes où les vésicules séminales étaient à peine ébauchées. Chez Octolasium lacteum, il est plus fréquent; j'ai trouvé parfois chez cet hôte la première paire de vésicules séminales complètement remplie de Grégarines si étroitement tassées les unes contre les autres qu'elles emplissaient toute la lumière et que toutes les cellules sexuelles avaient absolument disparu.

Lorsque le parasite est situé dans le cœlome, il est, ou totalement libre ou entouré par un repli de la membrane péritonéale et rattaché par un double feuillet soit à la paroi intestinale, soit à la paroi des néphridies. On n'en observe que très rarement qui soient contenus dans des kystes phagocytaires, avec des soies de l'hôte, des Nématodes, etc., etc.; au contraire, les kystes du parasite sont presque toujours englobés dans de tels kystes phagocytaires. Ces faits avaient été remarqués déjà par BosanqUET.

Dimensions, forme, Mouvements. - M. herculea est sphérique ou ovoïde (fig. XLVII), son diamètre peut atteindre jusqu'à 3 ou $4 \mathrm{~m} / \mathrm{m}$; elle présente des mouvements très lents du cytoplasme à son intérieur, mouvements à peine perceptibles et qui n'affectent pas sensiblement la forme extérieure.

Il n'y a pas de différenciation antérieure épiméritique permettant d'orienter facilement le corps ; les pôles antérieur 
et postérieur sont marqués simplement par les points d'entrecroisement des stries épicytaires. Celles-ci sont très fines et visibles seulement sur les coupes. Elles sont en général rectilignes, cependant, dans quelques cas, elles dessinent des zig-zag assez compliqués à la surface du corps de la Grégarine.

Le sarcocyte, très épais n'est pas d'une structure uniforme

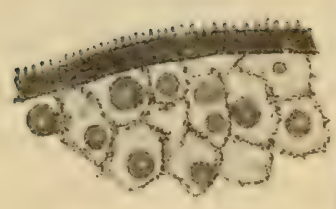

XLVTIT.

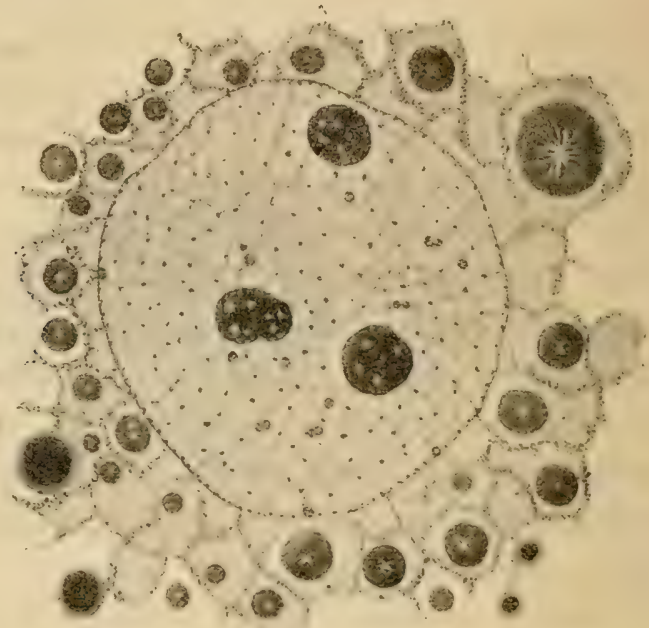

XLIE

FIG. XLVIII. Ectoplasme de $M$. herculea, coupe $\times$ 650, - FIG. XLIX. Noyau, grains de paramylon, masses chromatoildes de $\boldsymbol{M}$. herculea $\times 650$.

comme chez les autres Monocystis; les préparations colorées le montrent parsemé de petites zones claires, elliptiques, dispersées sans ordre apparent dans toute sa masse (fig. XLviII).

Les fibrilles du myocyte sont excessivement fines et difficilement perceptibles.

L'endoplasme paraît très sombre in-vivo, parce qu'il est bourré de grains de paramylon de grosseurs diverses, les uns très petits, d'autres au contraire très volumineux, pouvant atteindre jusqu'à 20 u de diamètre ; ces gros grains paraissent homogènes in-vivo, mais dans les préparations fixées et colo- 
rées, on aperçoit en leur centre une masse étoilée, que BosaNQUET considère comme un cristal, mais qui pourrait fort bien n'être qu'une simple cavité (fig. XLIX). Ces grains de réserve sont contenus dans les mailles d'un réseau cytoplasmique dont les travées paraissent formées de fins filaments sur lesquels sont disposées des séries de petites granulations fortement colorées par l'Orange.

Outre les grains de réserve, on observe encore dans l'endoplasme des masses chromatoïdes analogues à celles que j'ai signalées précédemment chez $M$. agilis. Par contre, je n'ai pas retrouvé les "capsules " décrites et figurées par BosaNQUET.

Le noyau, en général central, paraît sphérique sur le vivant, mais il est presque toujours déformé par l'action des fixateurs. Il est entouré d'une membrane épaisse à laquelle sont accolées de nombreuses granulations chromatiques, ot il renferme des karyosomes sphériques volumineux, en général au nombre de deux, mais parfois jusqu'à 4 ou 5 et qui possèdent la structure vacuolaire décrite par Bosanquet (fig. XLIX).

Le noyau est parcouru par un réseau très serré de fins filaments lininiens sur lesquels sont dispersées de nombreuses granulations de deux sortes : les plus volumineuses sont colorées en rouge par la Safranine-Gentiane-Orange, tandis que les petites, beaucoup plus nombreuses sont colorées en orange.

Les kystes de $M$. herculea, presque toujours contenus dans les grands amas phagocytaires des derniers segments du corps de l'hôte renferment les uns des sporocystes de $30 \mu$ sur $12 \mu$, les autres des sporocystes de $15 \mu$ sur $5 \mu$.

Le développement de cette espèce m'est complètement inconnu.

\section{Monocystis Cognettii n. sp.}

Nous avons rencontré dans les vésicules séminales de divers Helodrilus chloroticus Sav. récoltés dans les serres du Jardin des Plantes de Grenoble une curieuse Monocystidée, non décrite, que nous nommons $M$. Cognettii, la dédiant à M. Cognetti de 
Martiis, assistant au musée d'Anatomie comparée de Turin, qui nous a fort obligeamment déterminé une série d'Oligochètes ayant servi à ce travail.

Voici la diagnose de cette nouvelle espèce :

Monocystidée ovoüde ou sphérique de 60 à 65 u. de diamètre ou de $70 \mu$ sur $50 \mu$, en moyenne. Cuticule présentant des sillons méridiens réguliers et des sillons parallèles, séparant une série de quadrilatères au centre desquels on remarque une petite élévation en forme de dôme. Sarcocyte épais; fibrilles de myocyte assez développées. Mouvements cependant peu sensibles. Noyau sphérique à un karyosome central.

Hôte : Helodrilus chloroticus Sav.

Habitat et stège. - Monocystis Cognettii habite les vésicules séminales des Helodritus chloroticus Sav. des serres du Jardin des Plantes de Grenoble. Ces Oligochètes sont probablement d'origine exotique, en tous cas, je n'ai retrouvé le parasite dans aucun des individus de la même espèce récoltés en France ; ceux-ci renferment par contre quelquefois une autre Monocystidée du genre Zygocystis, que nous décrirons plus loin. $M$. Cognettii est assez rare; quelques hôtes seulement étaient infestés sur une centaine qui ont été examinés.

Dimensions, forme, mouvement. - Le parasite est en général ovoïde, ou plutôt piriforme, le pôle postérieur étant presque toujours terminé en pointe. Ses dimensions atteignent en moyenne $70 \mu$ sur $50 \mu$. Parfois, il se contracte et prend la forme d'une sphère régulière, cependant, à l'un des pôles, on observe toujours un petit cône plus ou moins ratatiné, plus ou moins étroitement appliqué sur le reste du corps et qui représente la queue du parasite (fig. L).

Les mouvements sont rares et très lents.

Le pôle antérieur, est comme nous venons de le voir, arrondi, le pôle postérieur étant au contraire terminé en pointe. Le pôle antérieur est marqué par le point de croisement des sillons méridiens. 
La cuticule présente en effet un quadrillage caractéristique formé par l'entrecroisement de deux sortes de sillons dont les uns, longitudinaux, décrivent des méridiens très réguliers, et les autres, transversaux, sont au contraire parallèles. Les sillons méridiens sont larges, ils se colorent fortement par le Fer; aux points de rencontre avec les sillons transversaux qui sont moins développés, ils s'élargissent sensiblement. Ces sillons sont bien visibles sur les parasites au repos, quand ceux-ci se contractent, au lieu et place de ces sillons on observe au contraire des crêtes saillantes (fig. LI), la cuticule très élastique se soulève donc au niveau des sillons pour donner ces crêtes aiguës.

La présence des sillons amène la formation à la surface du parasite de rangées régulières de saillies quadrangulaires ou arrondies au centre desquelles s'élève un petit dôme, sorte de perle que le fer teinte d'une façon intensive. Ces ornementations donnent au para-

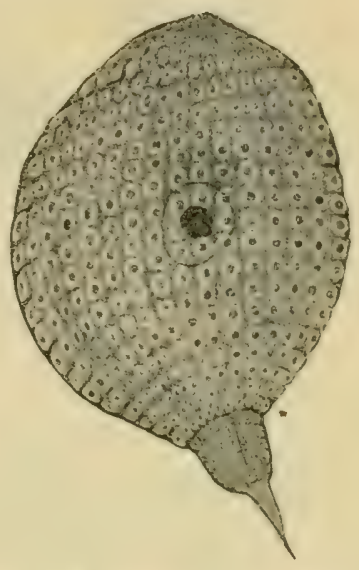

FIG. L. MI. Cognettii $\times 1000$. Ornementation de la cuticule. site coloré un aspect très curieux que l'on n'observe chez aucune des Grégarines connues jusqu'ici.

Le sarcocyte hyalin est bien développé ; il forme une couche d'épaisseur très inégale, beaucoup moindre au niveau des sillons, plus développée ailleurs.

Les fibrilles du myocyte sont surtout visibles dans les parasites contractés en sphère, où elles se présentent avec l'aspect décrit par PoRTer (1897) chez les Grégarines contractées de Rhynchobolus americanus Verrill, et que nous retrouverons chez $N$. anguillula. Le sarcocyte paraît ainsi découpé en une série de bandes claires séparées par des lames foncées, minces, correspondant aux fibrilles du myocyte (fig. LII). 
L'endoplasme est creusé d'alvéoles dans lesquelles sont logés les grains de paramylon. Ces alvéoles, plus petites à la périphérie augmentent de volume en allant vers le centre.

Le réseau spongioplasmique est formé de larges travées renfermant de gros grains fortement colorés par le Fer et des grains plus petits, éosinophiles.

Il semble y avoir ici, comme chez les diverses Grégarines étudiées à ce point de vue par LÉGER et DuBoscQ (1909) une

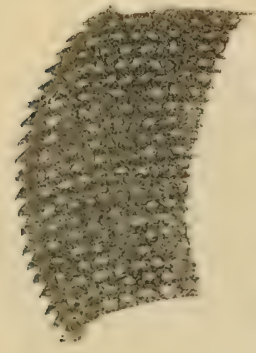

I. 1.

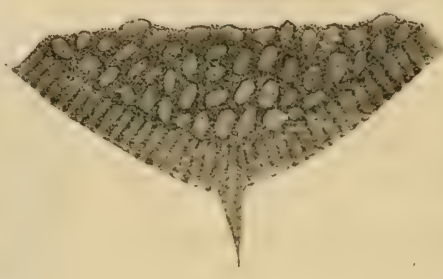

I. II .

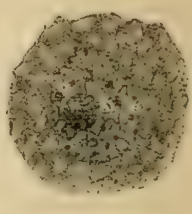

$L I I I$.

I'IG. LI. MI. C'ognettii × 1.300. Crêtes saillantes du parasite contracté. - Fia. LII, Queue de BI. Cognettii contractée; sarcocyte divisé en bandelettes; fibrilles du myocyte en noir, au voisinage de la cuticule $\times 1.300$. - FIG. IIII Noyau de M. Cognettii $\times$ 1.300 .

différenciation sexuelle qui se manifeste dans la structure des cytoplasmes. L'un des conjoints, la f d'après LÉGER et DuboscQ, présente de larges mailles avec de gros grains de paramylon, tandis que l'autre, le o a des mailles plus petites et des grains de paramylon plus fins.

Le noyau sphérique a un gros karyosome à structure vacuolaire ordinaire. Avec le Mann, il montre une vacuole centrale, une moelle rouge et une mince couche corticale violette ; parfois un ou deux grains plus petits sont accolés à sa surface. Un réseau de linine occupe le reste du noyau, dans les mailles duquel s'observent des grains rouges assez nombreux, tandis que quelques grains violets se trouvent sur le réseau, principalement au voisinage du karyosome (fig. LIII). 
La différence sexuelle déjà sensible dans les cytoplasmes des individus accouplés est plus manifeste encore dans les gamètes. On retrouve ici l'anisogamie découverte par BRASIL (1905 b) chez les Monocystis de Lumbricus herculeus ; les gamètes ơ sont plus petits, et ont un noyau condensé ; les gamètes ๑, plus volumineux, ont un noyau dilaté, plus riche en suc nucléaire.

Les sporocystes ne diffèrent pas, comme forme et comme structure de ceux des autres Monocystis des Lombriciens.

\section{Monocystis Duboscqi n. sp.}

On trouve assez communément dans les vésicules séminales et dans le colome de Lumbriculus variegatus O. Müll, aux environs de Grenoble, une Monocystidée remarquable par les processus singuliers de sa sporulation et que nous nommerons M. Duboscqi, la dédiant à M. le professeur Duboscq de l'Université de Montpellier.

Kölliker (1848) a signalé dans le même hôte une autre Monocystidée : M. saenuridis. Celle-ci diffère de notre parasite par sa forme très allongée et son noyau à karyosome unique; en outre, Kölliker ne mentionne pas chez elle le caractère vraiment très typique de $M$. Duboscqi, l'existence de sporozoïtes nus, se formant à la surface de sporocystes sans enveloppe différenciée.

Dragnose. - Monocystis ellipsoïdale allongée de $230 \mu$ sur $65 \mu$, présentant de vifs mouvements en sablier semblables à ceux de M. lumbrici. Ectoplasme mince à stries cuticulaires fines et serrées. Fibrilles du myocyte très ténues. Endoplasme à petites alvéoles régulières. Grains de paramylon de grosseur uniforme, sphériques. Noyau volumineux, sphérique ou ellipsö̈dal renfermant 2 karyosomes vacuolaires.

Sporocystes naviculaires, volumineux de $20 \mu$ sur $10 \mu$. Leur enveloppe est toujour's très mince, parfois même elle manque totalement et les sporozoïtes sont nus.

Hôte : Lumbriculus variegatus O. Müll. 
$M$. Duboscqi se rencontre dans les vésicules séminales et le cœlome de Lumbriculus variegatus O. Müll ; kystes et stades végétatifs sont visibles à travers les téguments du ver et apparaissent de l'extérieur comme des taches d'un blanc laiteux sphériques ou ellipsoïdales qui constellent parfois le corps sur toute sa longueur.

Le parasite a la forme d'un ellipsoïde étroit et allongé mesurant $230 \mu$ sur $60 \mu$ à $65 \mu$. Il est animé de mouvements en sablier tout à fait semblables à ceux de $M$. lumbrici, qui changent la forme extérieure comme l'indique la figure LV. Il n'existe pas de différence sensible entre les deux pôles.

L'ectoplasme très mince présente des stries cuticulaires longitudinales méridiennes peu développées. La couche de sarcocyte est fort peu épaisse. Les fibrilles transversales du myocyte peu marquées ne sont bien visibles que sur les coupes.

L'endoplasme finement granuleux est creusé d'alvéoles très régulières, petites, renfermant des grains de paramylon sphériques de grosseur uniforme. On observe ça et là dans le spongioplasme des masses chromatoïdes irrégulières parfois assez développées; d'autres grains chromatoïdes, plus petits, sphériques, beaucoup plus nombreux sont dispersés dans toute la masse de l'endoplasme.

Le noyau très volumineux, sphérique ou ellipsoïdal de 30 à $35 \mu$ de diamètre ou de $40 \mu$ sur $30 \mu$, renferme constamment deux gros karyosomes vacuolaires présentant chacun un grain accolé à leur surface. On observe souvent ici une vacuole qui fait saillie à la surface du karyosome, rappelant les figures données par Berndt (1902) et Kuschakewitsch (1907) chez les Grégarines de l'intestin de Tenebrio molitor. Il existe en outre un réseau de linine difficile à mettre en évidence et sur lequel on observe peu ou pas de chromatine.

Les parasites s'accouplent par un pôle; ils présentent pendant assez longtemps après leur union des mouvements très vifs, puis ceux-ci cessent, les deux individus se contractent et la paroi kystique est secrétée; elle est toujours résistante 
quoique peu épaisse, et très difficilement pénétrable aux réactifs.

Les kystes sont des ellipsoïdes de $300 \mu$ sur $180 \mu$ en moyenne, qui présentent fréquemment à leur surface une ornementation formée de poils ou d'épines fines et courtes. (Fig. LviI).

A l'intérieur, les noyaux se multiplient par mitose. Les

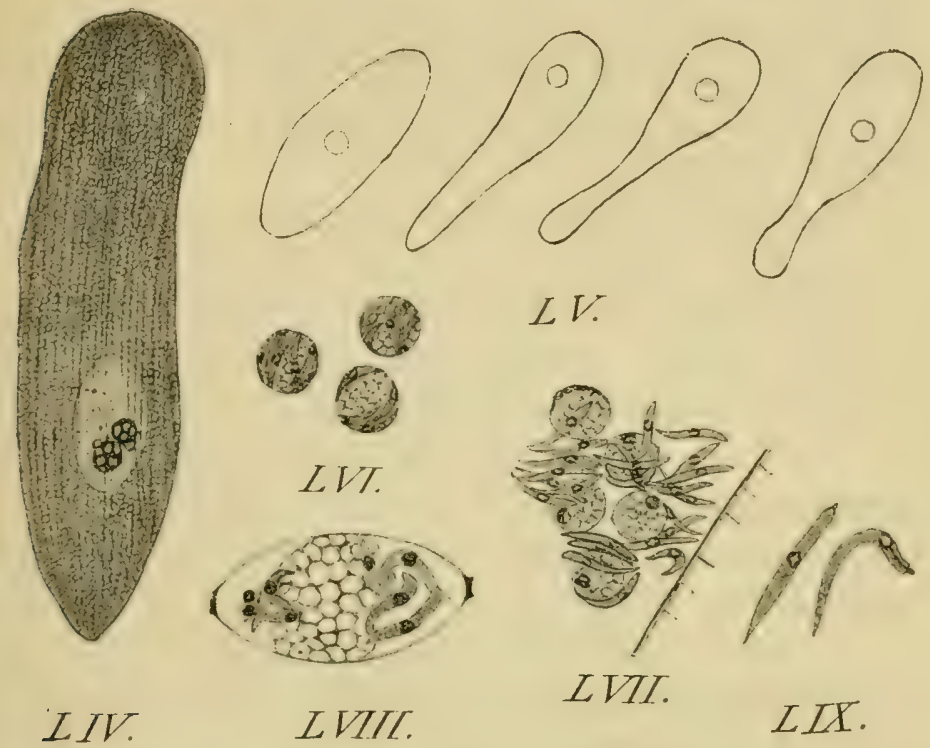

FIG. LIV. $\boldsymbol{M}_{\text {. Duboseqi }} \times 250$, - FIG. LV. Schéma montrant $\boldsymbol{M}$. Duboscqi én mouvement. FIG. LVI. MI. Duboseqi. Sporocystes sphériques sans enveloppe $\times 600 .-$ FIG. LVII. M. Duboscqi. Sporozoites libres dans l'intérieur du kyste. Reliquats sporocystaux sphériques à côté d'eux $\times 600$. - FIG. LVIII. Sporocyste de M. Duboscqi $\times$ 1.300. Paroi très mince. - Fig, LIX, $M$, Duboscqi. Deux sporozoiltes $\times 1.300$.

centrioles sont entourés par une masse archoplasmique très volumineuse. Le cytoplasme se découpe en boules de plus en plus petites, et finalement le kyste est rempli de sphères mononucléées, toutes semblables. Il n'y a donc pas ici anisogamie. Je n'ai du reste pas observé la copulation des gamètes.

Pour la formation des sporozoïtes, dans chaque sphère, le noyau se multiplie par mitose, les noyaux fils se portent à la périphérie et il s'individualise autour d'eux une zone cytoplas- 
mique qui se sépare du reste de la sphère. Ainsi se découpent tangentiellement les sporozoïtes (fig. LVI). Aucune enveloppe ne s'est constituée à la surface du sporocyste, les sporozoïtes sont libres dans le kyste (fig. LVII) quand ils se détachent de la boule cytoplasmique qui leur a donné naissance et qui apparaît le plus souvent dépourvue de noyau comme une masse de cytoplasme de reliquat.

Parfois la sphère s'allonge en fuseau, se couvre d'une mince enveloppe, et les sporozoïtes se forment dans l'intérieur comme chez les autres Monocystis. Mais la paroi est toujours fort peu épaisse (fig. LVIII).

On trouve parfois dans le même kyste des sporocystes pourvus d'une paroi et d'autres qui en sont dépourvus.

Les sporozoïtes sont des vermicules allongés, fusiformes de $14 \mu$ sur 1 à $2 \mu$, présentant une extrémité effilée, l'autre plus large est pourvue d'un court mucron. Le noyau placé en général au centre du corps a toute sa chromatine tassée sur la membrane, comme on peut le voir sur la figure LIX.

Les sporozoïtes paraissent se mouvoir librement dans le kyste, ils sont mis en liberté par la destruction de la membrane kystique, celle-ci est, comme nous l'avons vu, beaucoup plus résistante que chez les autres Monocystis, elle ne se laisse pas traverser par les réactifs et semble, à ce point de vue présenter certaine analogie avec la paroi des sporocystes ordinaires.

Je n'ai pas observé le développement du parasite.

\section{Rhynchocystis pilosa Cuénot.}

Cúnot (1901) donne la diagnose et la synonymie de cette espèce. D'après lui, $R$. pilosa aurait été vue et figurée par STEIN (1848), Sсhмidt (1854), Lieberkühn (1855), Ruschhaupt (1885) et Pfeiffer (1891). Nous sommes d'accord avec lui en ce qui concerne les quatre premiers observateurs; mais il nous semble que le dessin de Pfeiffer (1891, p. 38, fig. 8) représente une $M$. agilis intrablastophorienne et non $R$. pilosa ; par contre la figure 9 , page 39 de ce même auteur représente 
certainement des $R$. pilosa ; Pfeiffer les nomme $M$. magna et il dessine dans sa figure $9 a$ des parasites " mit dem Rest der mütterlicher Bechezelle an einen Pol " qui ne sont pas autre chose que des $R$. pilosa dont l'épimérite est piqué dans des blastophores en voie de dégénérescence; on se rend facilement compte du fait en comparant ces figures avec notre dessin (fig. 134). Pfeiffer n'a pas représenté les poils qui recouvrent le parasite, mais il arrive assez fréquemment qu'à ce stade les poils sont peu nombreux ou même absents.

La forme dessinée par Cú́not (1901, fig. 1) nous a paru être très rare; le parasite se présente le plus souvent sous l'aspect représenté figure $\mathrm{LX}, \mathrm{A}$.

Drzewecki (1903) a sans doute observé le même parasite, il l'appelle $M$. porrecta ; mais ses figures se rapportent manifestement à des $R$. pilosa adultes. De même que Pfeiffer, il paraît n'avoir pas observé les poils.

Brasil $(1905 b)$ a étudié aussi $R$. pilosa, mais seulement au point de vue des phénomènes sexuels.

Habitat et siège. - $R$. pilosa se rencontre communément dans les vésicules séminales des Lombrics : L. terrestris L., F. Müller, L. rubellus Hoffm., L. castaneus Sav. Le parasite jeune se développe dans les blastophores de l'hôte, comme $M$. agilis; lorsqu'il en est sorti, on le voit soit libre dans le fluide séminal, soit fixé parson épimérite à des blastophores en voie de dégénérescence.

Forme, dimensions, mouvements. - $R$. pilosa est très polymorphe; ce polymorphisme lui a fait attribuer beaucoup de noms différents par les auteurs qui l'ont observé : $M$. agilis, Stein (1848); $M$. agilis, Schmidt (1854, fig. 35); $M$. porrecta $M$. cuneiformis et $M$. minuta, RuschHaupt (1885). M. minuta est sans doute une forme jeune dans laquelle la queue est très pou développée.

Le parasite est d'abord ovoïde ou en forme de cœur ou de toupie, très rarement en fuseau ; lorsqu'il grandit, son extrémité postérieure peut se terminer en pointe : c'est la forme 
que Ruschinupt (1885), désigne sous le nom de $M$. cuneiformis; au contraire, cette extrémité peut rester mousse et arrondie; c'est le cas le plus fréquent. Sous cette forme la Grégarine a été appelée par Ruschнaupt : $M$. porrecta, Schmidt.

J'ai observé trois variétés principales distinctes non seulement par la configuration extérieure, mais encore par un ensemble de caractères cytologiques. Ces types sont représentés (fig. $\mathrm{Lx}, \mathrm{A}, \mathrm{B}, \mathrm{C}$,) nous les désignerons dans la suite sous les noms de forme $\mathbf{A}$, forme $\mathrm{B}$ et forme $\mathrm{C}$. Le seul examen des figures nous montre que $\mathrm{A}$ et $\mathrm{B}$ offrent de grandes ressemblances mais que l'aspect de $\mathrm{C}$ est notablement différent.

Les dimensions sont aussi variables que la forme : A et B atteignent à peu près la même taille : $500 \mu$ de long au maximum sur $15 \mu$ d'épaisseur dans la région la plus large; quant à $\mathrm{C}$, la longueur est en général plus courte, mais l'épaisseur atteint couramment 70 à $80 \mu$.

Ces diverses variétés ne coexistent pas toutes dans un même hôte; A est très commune chez Lumbricus terrestris L., Müll. ; $\mathrm{C}$ se rencontre aussi chez cet Oligochète, mais beaucoup plus rarement, quant à $\mathrm{B}$, on la trouve fréquemment chez L. rubellus Hoffm. et $L$. castaneus Sav. où A est rare et $\mathrm{C}$ absent.

Tous ces parasites sont peu agiles ; les granulations cytoplasmiques se meuvent lentement d'un pôle à l'autre avec les grains de réserve et la forme extérieure est à peine affectée par ce mouvement.

Quelle que soit la variété considérée, elle présente à son pôle antérieur un appareil différencié qui rappelle d'une façon frappante l'épimérite de certaines Polycystidées. Cet appareil, très complexe chez les individus jeunes, paraît s'atrophier partiellement chez les formes âgées, mais il ne disparaît pas complètement.

On a signalé chez un certain nombre de Monocystidées des formations épiméritiques plus ou moins compliquées; nous avons parlé déjà de l'appareil fixateur de Lankesteria ascidiae R. Lank., en le comparant au mucron antérieur de Monocystis lumbrici 
et $M$. agilis des Oligochètes; le bouton de Schaudinnella henleae Nusbaum (1903) parasite de l'intestin de Henlea leptodera Vejd. présente également avec ce mucron de grandes analogies. On a décrit aussi des productions semblables chez les Monocystidées des vésicules séminales. Ed. Perrier (1881) a observé chez une Grégarine parasite de Pontodrilus marionis un épimérite allongé, terminé par une couronne de crochets (fig. 48 D. pl. XVIII) qui ressemble beaucoup à celui de quelques Polycystidées, Corycella armata Léger, par exemple; LÜHE (1904) a vu chez Urospora saenuridis Köll. (1) uneformation analogue à celle de Lankesteria ascidiae; Sснмпт (1854) signale à İa partie antérieure de Monocystis magna une saillie conique plus ou moins allongée, enfin Ruschiaupt (1885) décrit dans plusieurs Monocystis des Lombrics des « bosses " parfois assez accentuées (fig. 1, 2, 3, 4 etc.) qu'il a même vues s'allonger parfois en trompe chez sa $M$. cuneiformis (fig. 7). Cette trompe est plus hautement différenciée que ne l'a supposé cet observateur, non seulement elle se rapproche par sa forme complexe de l'épimérite des Polycystidées, mais encore elle peut jouer à un moment donné comme celui-ci le rôle d'appareil fixateur. Stein (1848) et CuÉnot (1901) représentent le parasite avec une éminence conique en avant, mais ils ne signalent pas la diversité de formes que peut présenter cette pointe.

De même que chez $M$. lumbrici et $M$. agilis, il existe donc à l'extrémité antérieure de $R$. pilosa une zone de cytoplasme différencié, dense, dépourvu de toute inclusion, à granulations si fines et si serrées qu'il paraît presque homogène. Cette zone fait saillie sous forme d'un mucron conique non recouvert par les formations ectoplasmiques. Elle est beaucoup plus volumineuse que chez les espèces précédentes et elle est surtout remarquable par la diversité d'aspects qu'elle peut présenter.

Parfois elle prend la forme d'un cylindre assez allongé, étroit, terminé par un petit bouton légèrement renflé, pointu en avant qui correspond au mucron des espèces précédemment étudiées.

(1) Il s'agit de l'espèce que nous nommons $U$. tulificis. 
Autour du cylindre, laissant à nu seulement le mucron terminal, le sarcocyte présente une épaisseur assez considérable; il est creusé de sillons profonds délimitant des crêtes tranchantes qui se continuent sur le reste du corps par les striations épicytaires. Ces côtes saillantes parallèles sont sensiblement rectilignes quand le col est allongé ; si ce col est court au contraire, elles sont très fortement sinueuses (fig. 19). C'est l'aspect que l'on observe chez les parasites âgés et fortement allongés. Chez les Grégarines jeunes qui ont le plus souvent la forme d'une toupie, l'épimérite n'est jamais allongé ainsi en trompe ; il présente trois formes principales.

La partie antérieure renflée en dôme sphérique est creusée à son sommet d'une cavité peu profonde au fond de laquelle fait saillie le mucron hyaloplasmique. La couche de sarcocyte est uniformément répandue sur les bords de cette cavité et les côtes dessinent des courbes méridiennes régulières. La figure 22 représente l'appareil vu de face.

Quelquefois, la cavité n'existe pas, le mucron fait légèrement saillie au pôle antérieur; la couche de sarcocyte occupe une surface moindre et elle est plus épaisse, les côtes épicytaires sont encore disposées comme des méridiens, mais elles sont ondulées (fig. 28). La zone de hyaloplasme est disposée comme une sorte de calotte appliquée sur la région antérieure du parasite, elle est étalée sur une assez grande surface. Elle peut se rassembler en une boule placée à la base du mucron et incluse en grande partie dans l'endoplasme, le mucron ne présentant pas une saillie très prononcée ; dans ce cas, la couche de sarcocyte montre l'aspect représenté par la figure 24. L'épimérite rappelle alors tout à fait ceux que LÉGER (1894) a représentés chez Schneideria (pl. II, fig. 8) et chez Astero phora (pl. XII, fig. 11).

La séparation entre l'endoplasme dense et homogène de l'épimérite et l'endoplasme granuleux chargé d'inclusions formant la masse du corps du parasite est une surface courbe sensiblement régulière et dont la concavité est située en avant lorsque le mucron est peu saillant. Dans le cas où l'épimérite 
s'allonge en une trompe cylindrique cannelée, la concavité est au contraire tournée vers l'arrière, la plus grande partie de la masse de cytoplasme hyalin se réfugie vers les bords et l'endoplasme granuleux s'avance en cône dans l'axe de la trompe.

Le manchon de sarcocyte qui entoure le hyaloplasme épiméritique est très développé dans les formes $\mathrm{B}$ et $\mathrm{C}$; dans les formes A jeunes, il est assez épais, mais son importance diminue dans les parasites plus âgés.

Nous avons dit plus haut que cet épimérite pouvait en certains cas jouer un rôle identique à celui des Polycystidées. On rencontre en effet assez fréquemment des parasites piqués par leur pôle antérieur dans les blastophores dont ils tirent leur nourriture; les cellules sexuelles de ces blastophores situées autour de la base de l'épimérite s'appliquent étroitement contre le manchon sarcocytique strié, épousant les sinuosités des sillons et des côtes, tandis que celles du côté opposé gardent leur situation radiale normale et sont moins altérées dans leur forme (fig. 134). Pfeiffer (1891) ainsi que nous l'avons dit déjà, semble avoir observé ces Grégarines à tête enfoncée dans un blastophore, e'est du moins ce que paraissent indiquer ses figures 9 a et 9 b (p. 39) un peu trop schématiques. Il considère ces parasites comme des $M$. magna coiffées par les restes de "Becherzellen " auxquelles elles étaient tout d'abord fixées. Pour Drzewecki (1903) la fixation se ferait dans les cellules qui constituent les parois des vésicules séminales, nous n'avons jamais observé ce mode particulier de fixation.

Quoi qu'il en soit, l'épimérite de $R$. pilosa sert manifestement à la fixation du parasite, et très probablement aussi à sa nutrition, son rôle est ainsi identique à celui de l'épimérite des Polycystidées.

Les deux appareils sont done exactement comparables pour l'aspect et pour la fonction; mais il faut noter entr'eux une différence importante : chez les Polycystidées, la forme de 
l'épimérite est fixe, elle ne varie pas dans une même espèce (1); nous avons vu que l'épimérite de $R$. pilosa présente au contraire un métabolisme remarquable. Cette dissemblance est due à une différence de structure. LÉGER (1892) a noté qu'il n'y a dans l'épimérite des Polycystidées ni sarcocyte, ni myocyte; ces deux couches existent au contraire dans l'épimérite de $R$. pilosa, elles sont même plus développées là que sur tout le reste du corps, ainsi que nous le verrons; seul, le mucron terminal en est dépourvu, c'est donc ce mucron seul qui serait comparable d'une façon absolue à tout l'ensemble de l'épimérite des Polycystidées, le reste de la formation serait ainsi une sorte de protomérite très particulier, à cytoplasme différencié dont les granulations sont tellement fines et serrées qu'il paraît en quelque sorte hyalin, beaucoup plus dense par conséquent que le cytoplasme du deutomérite, mais non séparé de ce dernier par une cloison ectoplasmique. Le noyau qui est le plus souvent placé à la limite entre ces deux régions passe en effet facilement de l'une à l'autre sans effraction. Lorsque le parasite se détache de la cellule hôte, il n'abandonne pas dans l'intérieur de celle-ci son épimérite; cet appareil n'est donc pas transitoire comme celui des Polycystidées ; il ne paraît pas même tomber au moment de l'accouplement, il est alors fortement rétracté et fait à peine saillie au milieu du pôle antérieur.

L'épicyte qui recouvre tout le corps de $R$. pilosa est excessivement mince, il se déchire avec une grande facilité, c'est un caractère que l'on retrouve chez $M$. porrecta Schmidt où il a été observé déjà par Schмrdт (1854). Cet épicyte présente des striations longitudinales équidistantes assez visibles dans les formes B et C, moins sensibles, souvent même invisibles dans la forme A.

Nous avons vu que ces stries se soulèvent en crêtes très saillantes et aiguës dans la région de la trompe, là, elles sont tou-

(1) Quelques Polycystidées possèdent bien des épimérites de forme variable par exemple Pyxinia Möbuszi Léger et Duboseq ; mais il n'y a pas ici métabolisme de l'épimérite. L'épimérite primitif tombe et il est remplacé par un autre de forme différente (LEGGR ET DUBOSCQ, 1902) 
jours faciles à apercevoir, quelle que soit la forme considérée. Nous avons décrit les divers aspects qu'elles revêtent lors des changements de forme de l'épimérite.

Le sarcocyte est constitué comme chez les espèces précédentes par une substance dense et homogène, toujours dépourvue de granulations et qui forme au-dessous de la cuticule une couche dont l'aspect et l'épaisseur varient suivant la variété du parasite à laquelle on s'adresse.

Dans la variété $\mathrm{A}$, le sarcocyte s'accumule vers la région antérieure; nous avons décrit les divers aspects qu'il peut revêtir à la surface de l'épimérite. Sur le reste du corps il n'y a qu'une couche de sarcocyte excessivement réduite, souvent même imperceptible. La variété $B$ présente la même accumulation de sarcocyte au pôle antérieur, mais cette couche s'étend également sur le reste du corps où elle présente seulement une épaisseur un peu plus faible. Chez la variété C, il y a encore accumulation de sarcocyte au pôle antérieur pour la formation d'une couronne périmucronaire; mais au lieu de s'amincir brusquement à une faible distance de la base de l'épimérite, cette couche s'atténue peu à peu pour devenir dans la région postérieure aussi mince que dans la variété $\mathrm{A}$.

Les fibrilles du myocyte sont très peu développées chez $R$. pilosa; on ne peut les mettre en évidence avec une certaine netteté que dans la région de la trompe, surtout vers la base de celle-ci. Sur tout le reste du corps, le myocyte n'est pas perceptible. C'est sans doute à ce très faible développement du myocyte qu'il convient d'attribuer le manque presque complet de mobilité du parasite.

Il faut signaler comme dépendance de l'ectoplasme le revêtement pileux qui recouvre le corps de la Grégarine. Les poils de $R$. pilosa s'insèrent sur les stries épicytaires, ils sont très serrés chez les Grégarines jeunes, puis ils s'éclaircissent et même disparaissent totalement chez les individus âgés; on n'en rencontre qu'assez rarement dans la région épiméritique (fig. 21), mais ils recouvrent tout le reste du corps. Ce sont parfois 
des expansions filiformes, minces et très allongées, telles que Cúnot (1901) les a représentées, fig. 1. On peut les considérer alors comme de simples ornementations de l'épicyte, c'est l'aspect qu'ils offrent le plus souvent chez les formes A (fig. 21. 24).

D'autres fois, ils sont plus épais; leur base est alors une sorte de cône allongé dont la base peut présenter jusqu'à $3 \mu$ de diamètre, ou une courte baguette renflée en son milieu, parfois moniliforme, qui se continue par un fin filament semblable aux poils de la forme précédente (fig. 27). Le sarcocyte dans ce cas paraît concourir à leurformation. Telle est la structure la plus fréquente chez la variété $B$. Les poils de la région antérieure sont dirigés vers l'avant (fig. $\mathrm{Lx}, \mathrm{B}$ ); en arrière, ils se redressent peu à peu de façon à devenir normaux à la surface; vers la partie postérieure du corps, ils ont une direction récurrente.

Dans la variété $C$, on observe les mêmes faits; mais les poils de la région antérieure sont souvent plus élargis encore à leur base, une faible quantité d'endoplasme pénètre même parfois dans cette base, l'axe du poil étant ainsi occupé par un cytoplasme granuleux vacuolaire, peu à peu le diamètre de cette base diminue, à mesure que l'on se rapproche du milieu du corps et le poil n'est plus formé que de sarcocyte, il devient ensuite aussi fin que chez la variété A. Chez celle-ci, on observe quelquefois aussi des poils larges, coniques renfermant du cytoplasme vacuolaire (fig. 31), ils recouvrent alors tout le corps et la Grégarine prend un aspect tout particulier représenté dans la figure 30 .

L'entocyte a une structure aussi variable que l'ectoplasme, son aspect est en général semblable chez les variétés A et B ; mais il diffère pour C. La différence provient surtout de la forme des grains de paramylon.

Le cytoplasme alvéolaire est formé de grains de diverses grosseurs, en général plus grands que chez $M$. lumbrici et $M$. agitis, et qui sont disposés en un réseau plus ou moins régulier aux nœuds duquel s'observent des grains sidérophiles plus volumineux. Dans les alvéoles sont contenus les grains de 
réserve; ceux-ci sont elliptiques et de volume variable chez les variétés $A$ et $B$; chez $C$, ils sont sphériques, et, en général plus gros et très serrés. Parfois chez $A$ et $B$, on rencontre dans l'extrémité postérieure effilée des grains de paramylon très gros et sphériques présentant en leur centre une cavité assez développée; ces grains particuliers sont toujours peu nom breux.

Les grains sidérophiles que nous avons signalés aux nœuds du réseau cytoplasmique paraissent s'agglomérer en certains points de façon à constituer des masses assez vo$A$.

$B$

C.

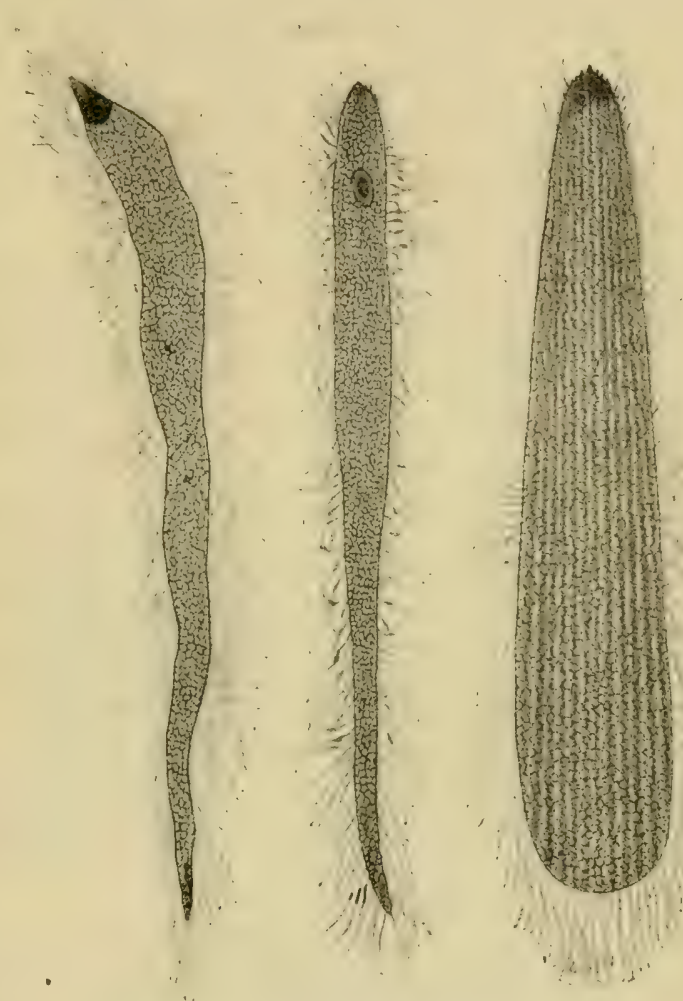

FIG. LX. Les 3 formes de Rhyncocystis pilosa $\times 300$.

\section{lumineuses}

qui atteignent parfois des dimensions égales ou même supérieures à celle du karyosome et que l'on pourrait considérer comme des formations chromidiales. Mais nous n'avons pu établir d'une façon certaine leur origine nucléaire. Dans les variétés A et B, l'une de ces masses est très constante, on l'observe toujours dans la queue, au voisinage de l'extrémité postérieure; elle 
paraît contenue le plus souvent dans une sorte de vacuole (fig. 25).

D'après ce qui précède, on peut résumer en quelques lignes les caractères morphologiques et cytologiques des trois variétés de $R$. pilosa.

VARIÉTÉ A. - Aspect d'une longue racine pivotante (carotte ou navet); corps recouvert de poils fins, épais chez les individus jeunes, plus rares ou même absents chez les parasites âgés. Accumulation de sarcocyte autour de la trompe pour former une couronne métabolique; couche de sarcocyte très mince sur le reste du corps. Stries épicytaires peu visibles. Grains de paramylon elliptiques et assez serrés (figure LX, A).

VARIÉTÉ B. - Aspect d'un fuseau peu renflé, à extrémité antérieure plus épaisse que l'extrémité postérieure. Poils larges, renfermant du sarcocyte et même de l'endoplasme, toujours assez serrés; ceux de la région antérieure dirigés vers l'avant. Couronne de sarcocyte autour de la trompe et sarcocyte bien visible sur tout le corps. Stries épicytaires très nettes. Grains de paramylon elliptiques, assez serrés (fig. Lx, B).

VARIÉTÉ C. - Corps cylindro-conique à extrémité antérieure plus mince. Présence d'une couronne de sarcocyte autour de l'épimérite. Stries épicytaires visibles sur tout le corps. Revêtement de poils complet: les poils de la région antérieure à base large, semblables à ceux de la variété $\mathrm{B}$, ceux de la région postérieure longs et filiformes comme chez A. Grains de paramylon sphériques et très serrés (fig. $\mathrm{Lx}, \mathrm{C}$ ).

Entre ces types, il existe toute une série de termes de passage, surtout entre les deux premiers. Ainsi, on trouve des formes A nues, à stries épicytaires très accentuées sur tout le corps, à couronne épiméritique presque nulle, des formes $\mathrm{B}$ à poils fins et à stries épicytaires peu visibles. La variété C, paraît s'éloigner un peu des deux autres, il n'existe pas entre elle et A et B des types de passage aussi nets et aussi nombreux; mais la présence de sa différenciation antérieure tout à fait analogue à la trompe de A permet de la considérer comme appartenant à la même espèce. 
Dans tous les parasites, à quelque variété qu'ils appartiennent, la structure du noyau est assez homogène. Il est toujours situé dans l'axe du corps, tout près du pôle antérieur, sauf dans les formes jeunes où sa situation est variable. Il so trouve à la limite entre l'endoplasme différencié de la trompe et l'entocyte granuleux du reste du corps, parfois il passe totalement dans l'endoplasme épiméritique ; s'il s'éloigne un peu de la surface de séparation de ces deux régions, le cytoplasme de l'épimérite envoie jusqu'à la membrane nucléaire un court prolongement canaliculaire; de cette façon, il y a toujours contact entre le noyau et l'endoplasme épiméritique.

Le noyau est assez volumineux, parfois sphérique, mais le plus souvent ovoïde, il est entouré par une membrane peu colorable, paraissant parfois discontinue, comme formée de petits grains placés les uns à côté des autres.

Le karyosome, volumineux, est central ou excentrique et situé toujours du côté opposé au mucron fixateur ; parfois, il est tangent à la membrane nucléaire. Il peut être sphérique ou irrégulier. Rarement il y a plusieurs karyosomes.

Le noyau est parcouru par un réseau de linine peu serré, difficile à mettre en évidence et dans les mailles duquel s'observe un coagulum granuleux. Il y a peu ou pas de granulations chromatiques sur le réseau.

Développenent. - Les stades jeunes sont intracellulaires. La puissance de pénétration du sporozoïte est plus considérable que chez $M$. agilis; on peut voir en effet des parasites très peu développés dans l'intérieur de spermatosphères qui ont presque achevé leur évolution; la Grégarine est logée, soit au centre du blastophore, soit au voisinage de sa périphérie. Dans le premier cas, elle ne sort de ce blastophore qu'après en avoir absorbé toute la substance; dans le deuxième cas au contraire, il semble qu'elle peut de bonne heure faire hernie en dehors, tout en restant fixée à la cellule hôte par son épimérite.

Le plus petit parasite que j'ai rencontré était ovoïde et 
mesurait $4 \mu$ sur $3 \mu .5$; son noyau sphérique, de $2 \mu$ de diamètre présente au centre un karyosome de près de $1 \mu$. de diamètre, entouré par une large zone claire autour de laquelle se voit un cercle de petites granulations chromatiques.

Dans le cytoplasme granuleux, on aperçoit, vers le pôle le plus étroit un grain fortement coloré.

Presque dès le début, les jeunes $R$. pilosa se distinguent facilement des jeunes $M$. agilis; le pôle le plus étroit se termine en effet en pointe, et de très bonne heure, le mucron antérieur apparaît sous forme d'une petite éminence conique de cytoplasme non granuleux; je l'ai observé sous cette forme chez un parasite de $10 \mu .5$ sur 9 \% 5 (fig. 132).

Le cercle de granulations chromatiques disposées autour du karyosome que nous avons rencontré chez les parasites très jeunes ne persiste pas comme chez $M$. agilis, il disparaît très rapidement. Le karyosome n'est pas uniformément coloré, on aperçoit en son centre une masse irrégulière, vacuolaire, fortement colorée par le Fer; le reste de la sphère karyosomienne s'imprégne au contraire par les colorants plasmatiques, Vert lumière, ou éosine (fig. 132). La membrane nucléaire est en général peu colorable, quelquefois cependant, on observe quelques grains chromatiques accolés à sa surface.

Les rapports entre le volume du corps et ceux du noyau et du karyosome sont à peu près les mêmes que chez $M$. agilis j'ai noté les dimensions suivantes :

\begin{tabular}{rlllll}
\multicolumn{3}{c}{ Parasite } & Noyau & Karyosome \\
$4 \mu$. & sur $3 \mu .5$ & $2 \mu$. & $0 \mu .75$ \\
$9 \mu .5$ & sur $4 \mu .5$ & $3 \mu .5$ & $1 \mu .75$ \\
$12 \mu$. & sur $10 \mu .5$ & $5 \mu .5$ & $2 \mu .25$ \\
$15 \mu$ & sur $12 \mu .5$ & $6 \mu$. & $2 \mu .5$ \\
$19 \mu$. & sur $15 \mu$. & $5 \mu .5$ & $3 \mu$. \\
$22 \mu .5 \operatorname{sur} 15 \mu$. & $5 \mu$.
\end{tabular}

Peu après la formation du mucron, on voit, dans la région qui entoure sa base, de petites crêtes triangulaires se soulever, 
ces crêtes représentent l'ébauche de la couronne de sarcocyte périmucronaire ; elles s'étendent longitudinalement en s'atténuant et dessinent des méridiens sur le corps du parasite. Peu après, des poils apparaissent sur ces crêtes; j'ai aperçu quelques-unes de ces formations très nettes chez un parasite intrablastophorien de $30 \mu$ sur $21 \mu$, autour duquel l'action des réactifs fixateurs avait amené la formation d'une petite cavité (fig. 133).

Le cytoplasme est plus dense, plus finement granuleux que chez $M$. agilis; il ne se creuse pas de vacuoles, mais de très bonne heure, il apparaît rempli de petites granulations sidérophiles (fig. 132, 133). Plus tard on aperȩoit des masses chromatophiles irrégulières, à surface mamelonnée, parfois entourées d'une vacuole et qui sont peut-être formées par l'agglutination des petites granulations sidérophiles. Au moment où les jeunes $R$. pilosa sortent du blastophore, leur cytoplasme est en général bourré de ces masses colorables qui restent longtemps visibles dans le corps du parasite. La plus grande partie finissent par s'émietter et disparaî̀re au moment de la formation des grains de réserve. L'une d'elles, assez volumineuse persiste presque toujours, nous l'avons signalée précédemment en décrivant le parasite adulte; elle se trouve dans le voisinage de l'extrémité postérieure qui s'est effilée en pointe. Son aspect rappelle beaucoup celui du karyosome, elle peut être formée d'une seule masse chromatoïde plus ou moins profondément lobée, ou de 3 ou 4 boules placées dans une vacuole; parfois elle se compose simplement d'une série de petits grains accumulés.

Chez certains parasites, formes intermédiaires entre $A$ et $C$, on peut observer dans cette région postérieure toute une calotte de cytoplasme dépourvu de grains de réserve, mais rempli de fines granulations fortement colorables par le Fer.

DrzeweCKI (1903) qui a décrit ces chromidies, les considère comme provenant de l'émiettement du noyau. Je n'ai pas observé comme lui de disparition du noyau à ce stade, pas plus 
que d'émission de chromatine nucléaire dans l'entocyte. Cependant cette émission est possible, elle se produirait sans doute comme chez $M$. agilis et donnerait les granulations chromidiales qui disparaissent au fur et à mesure que se développent les grains de paramylon et jouent probablement un rôle important dans la synthèse de cette substance de réserve. Les grains de para-

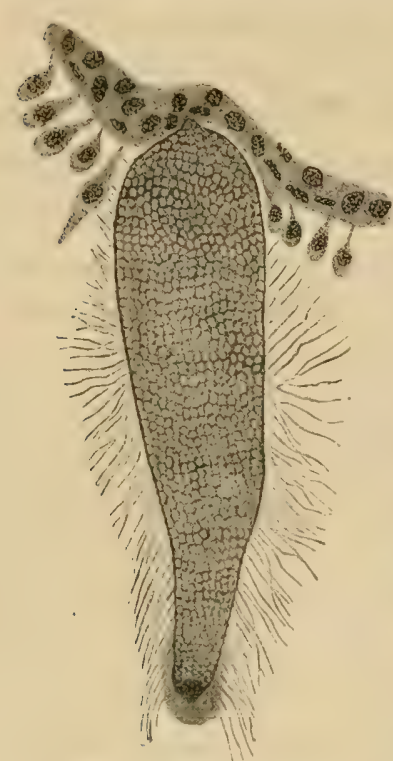

Fig. LXI. R. pilosa. Forme C, fixée par son pôle postérieur à la bordure marginale des pavillons séminaux. Demi schématique $x$ 100. mylon une fois formés ne disparaissent pas au cours de la vie végétative pour se reformer ensuite, du moins je n'ai constaté cette disparition que dans des cas de dégénérescence pathologique; ils sont utilisés après l'enkystement.

Le parasite s'accroît dans l'intérieur du blastophore, pendant que celui-ci se flétrit, en même temps que les cellules sexuelles qu'il supporte. Nous étudierons plus loin les diverses phases de cette altération.

Parfois la jeune Grégarine fait simplement hernie hors de sa cellule nourricière, son épimérite restant engagé dans celle-ci qui n'est pas encore complètement vide ; parfois au contraire, le parasite s'échappe et devient libre dans le fluide séminal. Il peut alors enfoncer son épimérite dans d'autres blastophores pour se nourrir à leurs dépens.

Certains parasites (variété C), situés dans le voisinage des pavillons séminaux accolent leur extrémité postérieure contre la paroi ciliée de ces pavillons, les poils à direction récurrente de cette région postérieure, s'engagent entre les cils et servent à maintenir en place la Grégarine dont l'extrémité antérieure renfermant le noyau flotte dans le liquide séminal qui emplit le pavillon. La figure LXI représente ce singulier mode de 
fixation. La figure 134 montre un parasite à fixation normale dont la trompe est piquée dans le blastophore nourricier, elle rappelle les figures 9 a et $9 \mathrm{~b}$ de Pfeiffer (1891).

$R$. pilosa ne présente jamais de mouvements bien vifs, et la forme extérieure du corps est rarement affectée par ces mouvements; parfois cependant, il se produit dans la région caudale des constrictions si profondes que cette région peut se séparer du reste du corps. Ce processus a été remarqué par RuschHAupt (1885) qui le considérait comme un processus d'enkystement préludant à la reproduction. C'est là une erreur, les fragments ainsi séparés ne tardent pas à dégénérer, ils sont du reste bien vite saisis et englobés par les phagocytes. Quant à la Grégarine mutilée, elle paraît continuer son évolution. Si la queue tout entière a été ainsi amputée, sa forme devient ellipsoïdale ou sphérique et elle rappelle alor's d'une façon frappante l'aspect de $M$. minuta Ruschhaupt, ou celle d'une Monocystidée arrondie et poilue trouvée par Schmid chez Lumbricus olidus : M. lumbrici olidi, décrite précédemment.

L'évolution du noyau est moins complexe que chez $M$. agilis. Je n'ai pas retrouvé non plus ici les émiettement, dissolution et néo-formation du noyau si minuticusement décrits par Drzewecki. Lorsque le noyau disparaît, e'est à la suite d'un processus pathologique qui sera décrit dans un chapitre spécial.

Drzewecki du reste a reconnu déjà le fait lui-même et remarqué que dans des conditions de vie défavorables, par exemple dans des vésicules séminales en voie de dégénérescence, le noyau disparaît souvent d'une façon totale. Ceci avait déjà été observé par Léger chez d'autres Grégarines.

Le noyau des individus très jeunes présente une membrane peu différenciée et un karyosome dont le centre est fortement coloré et la périphérie plus pâle, parfois une croûte mince plus foncée est visible à la surface (fig. 132, 133, 135).

Déjà à ce moment, le karyosome semble bourgeonner des grains chromatiques qui s'écartent de sa surface et se disper- 
sent dans le noyau; peut-être même passent-ils dans l'endoplasme. J'ai observé en effet des parasites chez lesquels la membrane nucléaire paraissait discontinue et à proximité des ouvertures, de gros grains chromatiques étaient visibles dans le cytoplasme; ce fait est, en réalité, assez rare.

Lorsque la Grégarine est devenue plus volumineuse, le karyosome se colore d'une façon à peu près uniforme, au centre comme à la périphérie ; mais il renferme toujours des vacuoles en nombre plus ou moins considérable. Sa surface est mamelonnée, comme recouverte par une multitude de petits grains en saillie; il peut même prendre un aspect mûriforme comme celui représenté par la figure 26 qui montre un noyau à deux karyosomes. Son aspect est alors tout à fait comparable à celui que présente généralement la chromidie postérieure.

Tant que les grains de paramylon sont peu développés, la situation du noyau est variable, cependant il est en général médian ; quelquefois, il est logé près de l'extrémité postérieure. Lorsque les grains de paramylon sont bien développés, le parasite s'est considérablement allongé et le noyau se rapproche du pôle antérieur, il vient se placer à la base du mucron, à la limite de l'endoplasme granuleux et de l'endoplasme épiméritique dense et homogène, souvent même, il émigre complètement dans celui-ci. Dans la variété B, le noyau cependant reste presque toujours à une certaine distance de l'extrémité antérieure.

Le karyosome est alors pâle, il présente seulement à sa surface quelques granulations colorées par le Fer. Sa situation dans le noyau est variable; il peut être presque médian, mais le plus souvent, il est logé très près de l'extrémité postérieure renflée; l'autre extrémité du noyau, dirigée vers le mucron s'allonge au contraire en pointe mousse. Le karyosome peut rester sphérique, mais il épouse en général la forme du noyau et devient ovoïde, sa pointe étant dirigée vers l'avant. Les figures $19,20,21,24$ représentent les divers aspects qu'il peut prendre. On observe fréquemment un gros grain ou un amas 
de petites granulations chromatiques au voisinage de son extrémité antérieure; souvent aussi, il y a accumulation de ces grains dans la pointe antérieure du noyau (fig. 21, 24).

Le cytoplasme du mucron antérieur demeure clair et anhyste mais le reste de l'endoplasme épiméritique devient fortement colorable, il se remplit de fines granulations chromatoïdes. On aperȩoit parfois en avant du noyau des grains colorés disposés en traînées longitudinales allant du mucron au noyau (fig. 21) et qui semblent indiquer en ce point l'existence d'une sorte de courant; il s'agit là vraisemblablement d'un processus nutritif. Parfois tout l'endoplasme de la trompe se colore avec une grande intensité par le Fer et il devient impossible de distinguer le noyau dans cette masse sombre. Ces phénomènes s'observent uniquement dans la variété $\mathrm{A}$ où la trompe est toujours plus développée.

De même que $M$. lumbrici, $M$. agilis et $M$. striata, certaines $R$. pilosa sont attaquées par des Bactéries qui y déterminent des phénomènes de dégénérescence étudiés plus loin dans un chapitre particulier.

Les Grégarines adultes sont libres dans les vésicules séminales de l'hôte où on les trouve pelotonnées sur elles-mêmes parmi les cellules sexuelles en voie de développement, souvent rassemblées en paquets volumineux, particulièrement chez L. rubellus. Quelques-unes restent accrochées comme nous l'avons vu par leur extrémité postérieure à la paroi ciliée des pavillons séminaux, au voisinage de leur bord.

La nutrition se fait soit par l'intermédiaire de l'épimérite différencié enfoncé dans les blastophores, soit par osmose à travers toute la surface; souvent sans doute par ces deux modes simultanément. Lorsque le corps est recouvert de poils larges et épais, renfermant de l'endoplasme, la surface d'absorption est considérablement augmentée, et l'on peut voir souvent ces poils recouverts d'une mulititude de granulations qu'ils semblent retenir agglutinées à leur surface et qui se colorent avec intensité par les colorants nucléaires. 


\section{Rhynchocystis porrecta Schmidt.}

Sснмпрт (1854) représente plusieurs Grégarines allongées avec une extrémité renflée; il identifie les unes (fig. 5, fig. 35) avec $M$. agilis Stein; je crois, avec Cú́not (1901) que c'est là une erreur; les autres (fig. 15) sont appelées $M$. porrecta. Celles-ci vivent en paquets dans les vésicules séminales de Lumbricus rubellus; leur noyau est le plus souvent logé dans l'extrémité antérieure renflée ; elles sont rares chez $L$. agricola. La figure de Sснмпрт est bien schématique pour permettre une comparaison approfondie, mais les caractères saillants qu'il donne pour ce parasite : étirement du corps, forme et situation du noyau, mouvements lents, très peu sensibles, fragilité de la cuticule, sont aussi des caractères propres à une Grégarine que nous avons trouvée fréquemment chez L. rubellus Hoffm. et que, pour ces raisons, nous identifions à l'espèce de Schmidt.

LteBeRkÜHN (1855) figure des Grégarines allongées (pl. I, fig. 1 et 5) que Cuḱnot considère comme des $M$. porrecta mais qui pourraient tout aussi bien représenter des $M$. magna Schmidt.

Ruschnaupt (1885), appelle $M$. porrecta Schmidt un parasite que je rattache à l'une des variétés de $R$. pilosa précédemment décrites. Nous avons vu que Drzewecki (1903) a sans doute commis la même confusion.

Brasil $(1905 b)$ déclare n'avoir vu que très peu de fois M. porrecta. Ce parasite est en effet rare chez Lumbricus herculeus Sav. $=$ L. terrestris L. ; Müll., uniquement étudié par cet observateur. Chez Lumbricus castaneus Sav. et surtout chez L. rubellus Hoffm., $R$. porrecta est beaucoup plus fréquent.

On rencontre ces Grégarines dans les vésicules séminales pelotonnées sur elles-mêmes et rassemblées en paquets assez volumineux, ainsi que l'avait déjà observé Schmidt. Elles ressemblent ainsi à $R$. pilosa; Schmidt avait noté aussi leur grande ressemblance avec $M$. magna.

On pourraitfacilement les confondre avec ces deux parasites 
mais un examen approfondi permet de reconnaître un assez grand nombre de caractère distinctifs.

Le corps de $R$. porrecta renflé en boule à l'extrémité antérieure se rétrécit brusquement à une faible distance de celle-ci pour aller ensuite en s'atténuant très peu jusqu'à l'extrémité postérieure, le parasite ressemble ainsi à un serpent à tête renflée. (fig. LXII).

Parfois ce renflement antérieur n'existe pas et la forme de $R$. porrecta est alors tout à fait semblable à celle des deux espèces voisines; cependant elle est plus régulière; presque cylindrique, elle est seulement un peu moins épaisse à l'extrémité postérieure qu'au pôle antérieur, et ne montre jamais de ces renflements séparés par des étranglements successifs qui donnent souvent au corps de $N$. magna un aspect moniliforme dû aux mouvements très vifs de ce parasite. Elle diffère aussi de la variété A de $R$. pilosa en ce qu'il existe toujours chez celle-ci une notable différence d'épaisseur entre la région antérieure et la région postérieure.

Les dimensions ne sont pas non plus les mêmes. Nous savons que $R$. pilosa ne dépasse pas $500 \mu$ de long; $R$. porrecta s'allonge beaucoup plus, sans cependant atteindre une taille aussi colossale que celle de $N$. magna. J'ai observé communément des $R$. porrecta de $2 \frac{\mathrm{m}}{\mathrm{m}}$ de long, avec une tête de $36 \mu$ d'épaisseur, et un

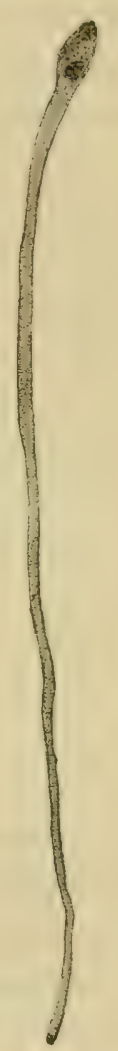

FIG. LXII, Rh, porrecúta. Forme ophi. céphale $\times 250$. corps de $28 \mu$ vers le cou et de 15 à $20 \mu$ vers le pôle postérieur.

Les mouvements sont très lents comme ceux de $R$. pilosa, le corps n'est pas déformé par ces mouvements comme cela se produit chez $N$. magna.

On observe au pôle antérieur un mucron très développé de cytoplasme hyalin, tout à fait semblable comme aspect et 
comme structure à celui de $R$. pilosa; mais il n'est pas entouré comme chez cette espèce d'une épaisse couronne de sarcocyte (fig. 23, 29).

L'ectoplasme est assez épais ; il comprend les trois couches ordinaires, épicyte, sarcocyte et myocyte.

L'épicyte très mince et peu résistant se rompt facilement entraînant la déchirure de tout l'ectoplasme qui laisse ainsi s'échapper les granulations entocytiques. Cet épicyte présente des stries longitudinales équidistantes, larges et assez serrées qui partent de la base du mucron où elles se soulèvent parfois en crêtes aiguës parfois très saillantes et qui s'atténuent rapidement en allant vers l'extrémité postérieure.

Le sarcocyte, dense, homogène, dépourvu de granulations forme une zone d'épaisseur à peu près uniforme sur toute la surface du corps.

Les stries du myocyte sont très serrées et excessivement fines.

L'entocyte est formé d'un cytoplasme dense, finement granuleux, dans lequel sont creusées des alvéoles où sont logés les grains de paramylon sphériques et en général très petits. Il renferme quelques granulations chromatophiles dont l'une, plus volumineuse, occupe comme chez $R$. pilosa le voisinage de l'extrémité postérieure.

Le noyau sphérique est très grand, il est toujours placé au voisinage de l'extrémité antérieure, que celle-ci soit renflée ou non. Il peut cependant exceptionnellement se trouver vers le milieu du corps, e’est ainsi que le représente Cú́Not (1901, fig. 3). Il occupe presque la totalité de la tête du parasite; de même que chez $R$. pilosa, sa région antérieure est tangente au cytoplasme épiméritique. Sa structure rappelle également celle du noyau de $R$. pilosa. La membrane est peu colorable; le karyosome sphérique ou irrégulier n'a pas de position constante; parfois, il a la forme d'un croissant irrégulier accolé à la membrane (fig. 29). Le reste du noyau est parcouru par un fin réseau de linine sur lequel sont distribuées quelques gra- 
nulations chromatiques; les mailles du réseau sont remplies par une sorte de coagulum granuleux dû sans doute à l'action des fixateurs.

Je n'ai pas observé le développement de cette espèce; il est possible même que ce ne soit qu'une simple variété non poilue de $R$. pilosa, car on observe entre ces deux parasites des formes de passage assez nombreuses; ils paraissent du reste avoir été confondus par bien des observateurs.

Il existe de même que chez $R$. pilosa des échanges très actifs entre le noyau et le cytoplasme de la trompe. Celui-ci est en effet souvent très fortement coloré par les colorants chromatiques; mais, alors que chez $R$. pilosa, le noyau disparaît le plus souvent dans cette masse sombre, ici au contraire, il reste toujours visible en arrière, avec sa constitution normale.

Je n'ai pas remarqué chez $R$. porrecta les phénomènes de dégénérescence si fréquents chez les autres Monocystidées des Lombrics, mais cela tient sans doute au petit nombre de parasites de cette espèce que j'ai eu l'occasion d'étudier.

\section{Nematocystis magna Schmidt.}

Hrstorique. - Cette grande et belle espèce de Monocystis a été découverte par Schnd (1854) dans les "Nebenhoden " de Lumbricus agricola. Certaines formes de Grégarines dessinées auparavant par Henle (1845) rappellent ce parasite; mais les figures de cet auteur sont trop schématiques pour que l'on puisse être certain de l'identification.

LIEBERKÜHN (1855, fig. 1 et 20) représente des Monocystis très grandes, cylindriques, allongées qui sont très probablement des $N$. magna.

D' Udekeli (1856) a observé des $N$. magna avec deux noyaux (pl. I, fig. 17).

BÜTSCHLI (1881) a retrouvé ce parasite en grande quantité dans un gros exemplaire de $L$. terrestris ; il a reconnu que la Grégarine est fixée à la paroi des pavillons ciliés par l'inter- 
médiaire de cellules spéciales, ciliées et en forme de coupe, les " pokalförmige Zellen ».

Ruschiaupt (1885) a rencontré lui aussi $N$. magna, mais il ne l'a pas étudiée en détail.

Wolters (1891), Bosanquet (1894), Clarke (1895), donnent des figures de l'accouplement et de l'enkystement du même parasite.

Cú́not (1901) a observé également $N$. magna, mais il a confondu sous ce nom deux espèces, et la figure 5 de son travail se rapporte manifestement à un autre parasite.

Drzewecki (1903) a étudié cette Grégarine au point de vue de l'évolution nucléaire pendant les stades végétatifs et BRAsIL (1905 b) a suivi cette même évolution durant l'enkystement, puis la formation des gamètes et leur copulation.

Diagnose. - Monocystidée de grande taille (jusqu'à $5 \mathrm{~mm}$.) ; corps vermiforme, très déformable, d'un blanc éclatant, dont l'extrémité antérieure est fixée à une cellule hypertrophiée, cupuliforme de la paroi des pavillons vibratiles des vésicules séminales. Cuticule finement striée en long; fibrilles transversales du myocyte bien visibles. La présence de ces deux sortes de striations donne ainsi à l'ectoplasme un aspect quadrillé très régulier. Noyau ellipsoïdal à un seul gros karyosome excentrique. Kystes volumineux, libres dans la cavité des segments génitaux ou renfermés dans les nodules phagocytaires des derniers anneaux.

HôTE : Lumbricus terrestris L., Müll.

HABItAT ET SIÈGe. - N. magna est fixée par son extrémité antérieure à la paroi ciliée des pavillons testiculaires de Lumbricus terrestris L., Müll., je ne l'ai jamais observée chez les autres Lombriciens; elle ne paraît pas non plus se trouver dans les vésicules séminales comme les autres espèces de Monoeystis.

Dimensions, forme, mouvements. - Cette Grégarine est la plus volumineuse des espèces que l'on rencontre chez les Lombrics; elle peut atteindre jusqu'à 4 à $5 \mathrm{~mm}$. de long, avec 
une épaisseur moyenne de 40 à $50 \mu$. Au repos, c'est un long cylindre dont les deux extrémités se terminent en pointe mousse. Elle a done la forme d'un Nématode ; mais son corps, excessivement contractile, est susceptible de s'allonger et de se raccourcir notablement, en diminuant ou augmentant son diamètre; parfois, il présente une série de renflements et d'étranglements qui lui donnent une apparence moniliforme; parfois elle a l'aspect d'une massue allongée à manche très grêle, parfois elle ressemble à un haltère ; la forme de la coupe transversale est le plus souvent régulièrement circulaire, mais elle peut varier aussi dans de très larges limites.

Je n'ai pu malheureusement étudier $N$. magna que in-vivo et sur des coupes sériées, cette méthode ne permet pas de se rendre très exactement compte de la morphologie externe du parasite.

J'ai déjà indiqué dans un précédent chapitre les ressemblances de $N$. magna avec divers autres parasites du Lombric. Sснмірт (1854) qui avait remarqué la ressemblance frappante de $N$. magna et de $R$. porrecta ne distinguait guère les deux espèces que par la différence d'habitat, j'ai relaté les divers caractères qui les séparent, ainsi que ceux qui éloignent $N$. magna de $R$. pilosa. La variété C de ce dernier parasite ressemble beaucoup à $N$. magna, elle s'en rapproche également par son habitat, puisqu'on la trouve fixée par son extrémité postérieure au bord libre de l'entonnoir cilié des pavillons-séminaux; mais nous savons qu'elle présente un épimérite particulier que nous ne retrouvons pas chez $N$. magna, non plus que le revêtement pileux. $N$. magna se rapproche aussi d'une espèce découverte par Cú́not (1901) chez All. terrestris et qu'il dessine (fig. 5) la confondant avec N. magna. Nous décrivons plus loin ce parasite en indiquant ses caractères différentiels.

Bütschli (1882) représente (Taf. xxıI, fig. $1 b$ ) des denticulations spéciales de la cuticule au pôle antérieur de N. magna et les considère comme des sortes de formations épiméritiques. Ce même observateur a montré que le parasite est implanté 
par son pôle antérieur pointu dans des cellules spéciales, les " pokalförmige Zellen " qui font saillie à la surface des pavillons séminaux ciliés. Ces denticulations représentent non pas l'épimérite mais simplement les restes de celui-ci, lorsque la Grégarine s'est détachée de la cellule-hôte.

Le parasite en effet a une partie de sa région antérieure logée dans une cavité cupuliforme ou infundibuliforme, creusée au centre de la cellule-hôte; les rebords de cette cavité sont resserrés de telle façon qu'ils produisent sur le corps de la Grégarine, une constriction plus ou moins accentuée, sorte de cou qui sépare du reste du parasite une tête arrondie et coiffée de sa cellule hôte comme d'une sorte de volumineuse calotte. Cette disposition joue certainement un rôle dans la fixation du parasite et contribue à le maintenir solidement attaché à la cellule nourricière ; cette fixation est assurée en outre par un engrénement très étroit des stries cuticulaires dans des rainures correspondantes creusées dans la paroi de la cellule-hôte et par un appareil épiméritique très particulier.

: Nous avons vu que, chez $M$. lumbrici et $M$. striata, les côtes ectoplasmiques se prolongeaient postérieurement en formant une touffe de poils assez longs, parfois ramifiés. Ici, le même phénomène s'observe au pôle antérieur; tout autour du mucron conique central piqué au centre de la cavité de la " pokalförmige Zelle "), et dont la structure est absolument comparable à celle du mucron des espèces étudiées précédemment (cytoplasme très dense, hyalin, fortement colorable, surface non recouverte par l'ectoplasme), on voit, chez certains individus, les côtes se prolonger et s'enfoncer dans la cellule nourricière (fig. 101). Je n'ai pu suivre ces formations sur toute leur longueur; elles sont entremêlées à un système de fibrilles très développées propres à la cellule-hôte et parmi lesquelles il n'est pas possible de les distinguer. Je ne pense pas cependant que ces prolongements soient très longs, il ne semble pas qu'ils puissent traverser la cellule de part en part et pénétrer jusque dans la profondeur de l'épithélium des pavillons. Ce ne sont 
là sans doute que des portions des côtes ectoplasmiques fortement étirées par la traction considérable qu'exerce sur elles le poids du parasite.

L'ensemble de ces prolongements épiméritiques et des fibrilles de la cellule-hôte constitue dans l'axe de celle-ci une sorte de trone fortement teinté par les colorants chromatiques (fig. 101), et qui emplit parfois complètement le pédoncule rattachant la cellule cupuliforme à l'épithélium. Au moment où il pénètre dans cet épithélium, le trone se résoud en ses fibrilles constituantes qui divergent, rayonnent en tous sens dans les régions adjacentes de l'épithélium et s'étendent souvent à des distances considérables.

L'endoplasme pénètre parfois dans ces racines épiméritiques piliformes comme il

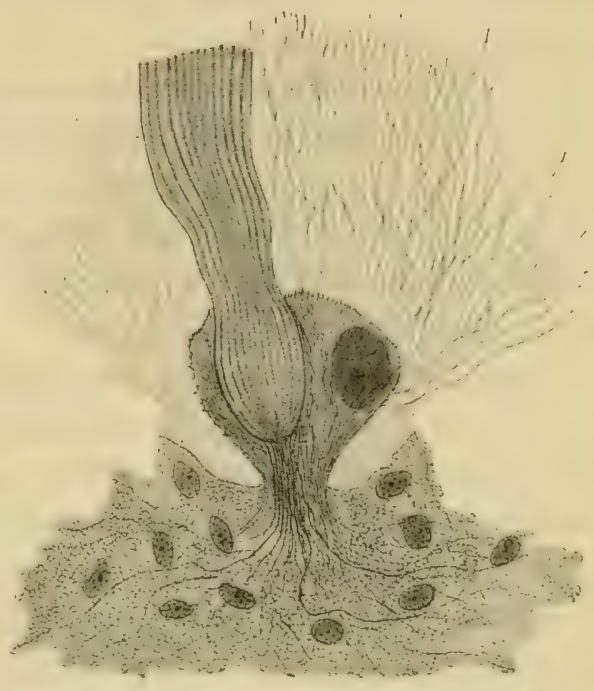

FIG.LXIII, $N$, magna à tête incluse dans une cellule cupuliforme $\times 350$. pénètre dans les poils de $R$. pilosa ; c'est même ce qui permet de déceler facilement leur présence parmi les fibrilles de la cellule support. Lorsque la Grégarine se détache, elle abandonne ces racines dans l'intérieur de la cellule-hôte, et il ne persiste de l'épimérite que les denticulations saillantes signalées par Bütschli (1882). N. magna possède donc un épimérite transitoire, tout à fait comme celui des Polycystidées, et dont la structure rappelle l'épimérite de Pogonites, Bothriopsis et Nina (= Pterocephalus). Cet épimérite n'est pas constant; dans un certain nombre de cas, je n'ai pas réussi à le mettre en évidence (fig. LXIII). 
Les côtes longitudinales ectoplasmiques, si accentuées dans la région antérieure où elles se prolongent en appendices fixateurs, s'atténuent souvent peu à peu vers l'arrière et so réduisent à de fines stries parallèles, simples ornementations de l'épicyte.

Le sarcocyte est bien développé ; dans la région antérieure il présente des bandes longitudinales d'épaississement qui correspondent aux côtes; postérieurement, son épaisseur est sensiblement uniforme sur toute la surface du corps du parasite.

Les fibrilles circulaires transversales du myocyte sont toujours bien visibles, et disposées parallèlement; elles sont très rapprochées et très fines. Il semble qu'il existe ici également des fibrilles longitudinales très fines elles aussi et très serrées ; on les aperçoit quelquefois entre les bandes d'épaississement sarcocytique.

L'endoplasme a la même structure que dans la variété C de $R$. pilosa. Les grains de paramylon paraissent cependant moins volumineux et moins serrés.

Le noyau est très grand, il a généralement la forme d'un ellipsoïde allongé ; mais les mêmes causes qui modifient le contour de la Grégarine et le rendent parfois irrégulier agissent aussi sur le noyau, qui prend par retentissement une forme également irrégulière.

La membrane nucléaire se colore par les colorants de la chromatine, mais avec une très faible intensité. A l'un des pôles du noyau s'observe un gros karyosome sphérique dont le centre est occupé par une ou deux grosses vacuoles et la zone périphérique par une série de vacuoles plus petites. A la surface existe la plupart du temps une couche plus fortement colorable (fig. 105).

D'après CuḱNo'T (1901), il y aurait plusieurs karyosomes, chez $N$. magna ; c'est une erreur due à ce que cet observateur a confondu avec $N$. magna une autre Grégarine parasite des pavillons ciliés des Helodrilus longus Ude et Helodrilus caliginosus Sav. Le noyau est parcouru par un réseau de linine 
assez serré sur lequel sont distribués quelques rares grains chromatiques.

De même que pour les autres espèces de Monocystidées des Lombriciens, je n'ai pas su distinguer d'une façon certaine les kystes et les sporocystes de N. magna; sans doute convient-il d'attribuer à ce parasite les volumineux kystes sphériques de $\frac{1}{2} \mathrm{~mm}$. ̀̀ $3 \mathrm{~mm}$. de diamètre que l'on rencontre dans la cavité générale de $L$. terrestris, au voisinage des pavillons vibratils séminaux et qui renferment des sporocystes de taille très constante dans un même kyste, mais variable avec les kystes considérés. Ces sporocystes mesurent en effet $25 \mu 5$ sur $9 \mu$, ou bien ils présentent des dimensions moitié moindres.

Développement. - Les stades jeunes de $N$. magna sont intracellulaires, ils sont contenus dans des cellules épithéliales de l'entonnoir cilié, hypertrophiées et faisant saillie dans la lumière du pavillon (fig. 98). Le plus petit des parasites que j'ai observés mesurait $21 \mu$ sur $10 \mu .5$; légèrement courbé en arc, il renfermait en son centre un noyau sphérique, volumineux, pourvu d'un karyosome excentrique, massif, peu colorable, accolé à la membrane et de nombreux grains chromatiques de diverses tailles épars sur un réseau lininien à mailles serrées. L'extrémité du parasite dirigée vers le pédoncule de la cellule-hôte présentait un petit cône de cytoplasme dense et fortement coloré qui est sans doute l'origine du mucron antérieur (fig. 98.)

La cellule épithéliale qui renferme les jeunes parasites fait hernie hors de l'épithélium auquel elle n'est plus rattachée que par un court pédoncule, son aspect rappelle tout à fait celui de la "pokalförmige Zelle " qui supporte les $N$. magna adultes, mais elle est moins développée (40 à $50 \mu$ sur 20 à $30 \mu$, au lieu de 80 à $100 \mu$ sur 40 à $50 \mu$.) et son bord externe non creusé en coupe supporte des cils allongés à large base conique.

De même que Lankesteria ascidiae R. Lank., la jeune N. magna fait hernie à un moment donné hors de sa cellule nourricière, qui continue à enserrer étroitement l'extrémité antérieure du 
parasite. Les côtes longitudinales qui ornent la surface de cette extrémité sont fortement enchâssées dans des rainures qu'elles ont formées dans le cytoplasme de la cellule-hôte (fig. 182), et elles assurent la fixation de la Grégarine. Sans doute sous l'influence de la traction exercée par le poids du parasite qui grandit considérablement, ces côtes s'étirent en filaments

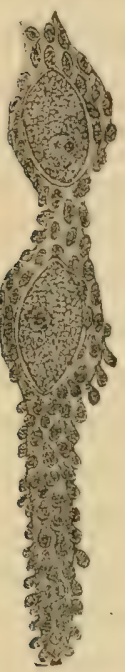

FIG. LXIV. Parasites inclus dans l'épaisseur de la paroi des pavillons séminaux $\times$ 100. qui restent enfoncés dans le centre de la cellule; celle-ci grandit et s'étire sous la même influence et bientôt se trouve atteint le stade que nous avons décrit pour le parasite adulte.

Schind (1854) a observé à la place des Nebenhoden d'un jeune Lombric des " traubenförmige Gebilde " dont il représente l'un des éléments, fig. 2, taf. xIv, et qui pourraient être d'après lui des formes jeunes de $N$. magna. J'ai retrouvé des formations représentées fig. LXIV qui sont probablement analogues aux " traubenförmige Gebilde " de Schmidt; pas plus que cet auteur je n'ai pu suivre le sort de ces singulières productions, et je ne sais comment les interpréter d'autant plus que je ne les ai étudiées que sur frottis, n'ayant jamais eu l'occasion de les retrouver sur des coupes sériées. Je ne crois pas cependant qu'elles puissent être rattachées au cycle évolutif de $N$. magna; cette Grégarine ne se présente pas en effet à l'état adulte en paquets serrés, de plus elle envahit les cellules pariétales et je n'en ai jamais remarqué qui s'enfoncent profondément dans les tissus de l'hôte, comme c'est le cas pour les parasites que j'ai observés après Schмidt.

Je n'ai pas rencontré de parasites chez $N$. magna comme chez les autres Monocystidées étudiées précédemment, mais ce fait tient peut-être au petit nombre de Grégarines observées. 
Nematocystis lumbricoides $\mathrm{n}$. $\mathrm{sp}$.

Nous retrouvons dans les vésicules séminales des Helodritus, de même que chez les Lombriciens deux types de forme de Monocystis, l'une voisine de la sphère, l'autre au contraire proche du cylindre. A l'une des espèces cylindroïdes nous avons donné le nom de $N$. lumbricoides.

Diagnose. - Corps cylindroïde aminci à ses deux extrémités et ayant la forme d'un Lombric. Taille assez considérable : jusqu'à $1 \mathrm{~mm} .5$ de long sur $60 \mu$ d'épaisseur. Mouvement des granulations cytoplasmiques analogue à celui de N. magna. Epicyte à striations longitudinales très fines et très rapprochées les unes des autres. Sarcocyte bien développé. Fibrilles du myocyte peu visibles sauf aux points où le parasite se contracte. Endoplasme alvéolaire avec grains de paramylon peu volumineux. Noyau ellipsoidal allongé renfermant généralement un seul karyosome.

Hôte : Helodrilus caliginosus Sav.

Nematocystis lumbricoides est assez rare; nous n'avons rencontré ce parasite que dans certains individus de Helodrilus caliginosus Sav. provenant des environs de Grenoble. Il habite les vésicules séminales. Il a la forme d'un fuseau allongé, peu renflé au milieu, s'atténuant insensiblement vers les extrémités et qui rappelle absolument l'aspect d'un ver de terre (fig. Lxv). Les dimensions sont à peu près les mêmes que celles de $R$. porrecta; le parasite est cependant un peu plus épais; il mesure jusqu'à $1 \mathrm{~mm}$. 5 de long sur $60 \mu$ de diamètre dans la région la plus épaisse. Le cytoplasme se meut dans l'intérieur du corps comme chez $N$. magna, mais ce mouvement n'affecte pas autant la forme extérieure, il se traduit simplement par des successions de renflements et d'étranglements peu marqués, figurant de petites ondes. En dehors de ce mouvement, le parasite peut se tordre et s'enrouler sur lui-même, absolument comme un Lombric qui s'agite dans la main qui le saisit. 
Il n'y a pas de différence appréciable entre le pôle antérieur et le pôle postérieur et il n'existe ni épimérite, ni mucron hya-

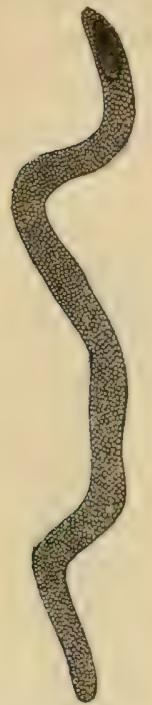

FIA. IXV. N. lum bricoides $\times 50$.

\section{loplasmique.}

L'épicyte est mince, orné de striations longitudinales parallèles, excessivement fines et très rapprochées les unes des autres.

La couche de sarcocyte assez développée a une épaisseur à peu près uniforme sur toute la longueur du corps, sa structure est la même que chez les autres Monocystidées.

Les fibrilles du myocyte sont aussi fines et aussi serrées que les stries cuticulaires; elles sont à peine perceptibles sur le parasite au repos; mais lorsqu'il se contracte et présente des mouvements de torsion, les fibrilles myocytiques deviennent très visibles, elles se présentent, en coupe optique comme de fines denticulations placées côte à côte à la limite de l'endoplasme et du sarcocyte. L'ectoplasme tout entier montre alors de très petits plissements en accordéon (fig. LXvi) qui correspondent aux denticulations du myocyte, de sorte qu'aux points de courbure, l'aspect du parasite rappelle un peu celui d'une lanterne vénitienne cylindrique en demi-extension.

L'endoplasme granuleux est creusé d'alvéoles irrégulières renfermant les grains de paramylon ellipsoïdaux et peu volumineux. Il ne paraît pas contenir de granulations chromidiales comme les autres Monocystidées, du moins n'en ai-je jamais observé.

Le noyau est assez volumineux ;

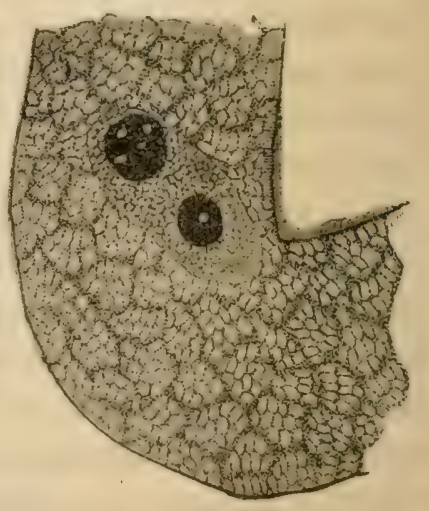

FIG. LXVI, $\boldsymbol{H}$. lumbricoides $\times 1000$ noyau à 2 karyosomes; plissements de l'cctoplasme en accordéon. 
c'est un ellipsoïde allongé dont la situation est variable; tantôt il est médian, tantôt rapproché de l'une ou l'autre des extrémités. Il contient un, très rarement deux karyosomes sphériques, présentant une ou deux grosses vacuoles centrales et une série d'autres, périphériques, plus petites. A la surface, on observe une mince zone plus colorée que le reste. Je n'ai aperçu ni réticulum lininien, ni grains chromatiques dans le noyau, il est rempli de fines granulations dues sans doute à la coagulation du suc nucléaire par les fixateurs.

Je ne connais rien du développement de cette espèce.

Nematocystis vermıcularis $\mathrm{n} . \mathrm{sp}$.

Les vésicules séminales de Helodrilus longus Ude renferment aussi une Grégarine allongée, voisine de $N$. lumbricoides, dont elle n'est peut-être qu'une simple variété. Je la désigne sous le nom de $N$. vermicularis $\mathrm{n}$. sp.

'Diagnose. - Monocystidée à corps fusiforme assez épais, présentant à peine $1 \mathrm{~mm}$. de long avec une épaisseur de $100 \mu$. Pôle antérieur orné d'une calotte formée de petits prolongements cylindriques placés côte à côte ; pôle postérieur recouvert de poils fins dirigés en arrière. Ectoplasme mince; pas d'ornementation cuticulaire visible. Endoplasme assez riche en granulations chromatoïdes. Noyau ellipsoïdal renfermant un seul karyosome.

Hôte : Helodrilus longus Ude.

$N$. vermicularis est plus commune que $N$. lumbricoides; on la rencontre dans les vésicules séminales et dans le cœlome de Helodrilus longus Ude. Au printemps, j'ai observé des individus adultes bien développés; en été et en automne, je n'ai rencontré que des individus jeunes complètement entourés par un manteau de phagocytes, ou des kystes à des stades avancés.

Cette espèce est voisine de $N$. lumbricoides, mais la forme des individus âgés est plus épaisse, plus massive. Alors que $N$. lumbricoides mesure jusqu'à $1 \mathrm{~mm}$. 5 de long, sans dépasser $60 \mu$ 
d'épaisseur, les exemplaires les plus développés de $N$. vermicularis que j'ai rencontrés n'atteignaient pas $1 \mathrm{~mm}$. tandis que leur diamètre n'était pas inférieur à $100 \mu$.

La forme du parasite ne peut être étudiée avec exactitude que sur certains exemplaires libres dans le cœlome, car dans

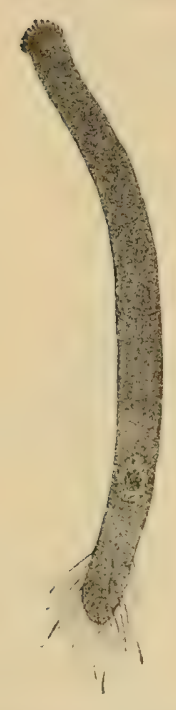

FIa. LXVII. N.vermicularis $\times 120$. Forme libre dans la cavité générale avec ses expansions antérieures et postérieures. les vésicules séminales, presque tous les individus sont recouverts de phagocytes.

Les individus du colome présentent à leur extrémité antérieure une sorte de calotte constituée par de courtes expansions en forme de bâtonnets ou de petites boules, disposées les unes à côté des autres et parfois assez serrées. Cette extrémitê antérieure est arrondie; l'extrémité postérieure, parfois légèrement acuminée est au contraire recouverte sur une certaine longueur de poils fins dirigés en arrière et plus ou moins allongés (fig. LXVII).

Dans les vésicules séminales, ces expansions des deux pôles sont très difficiles à voir parce que les phagocytes s'entassent entre elles et forment au parasite un étroit manchon.

L'ectoplasme est plus mince encore que chez $N$. lumbricoides; il comprend un épicyte et une faible couche de sarcocyte, le tout interrompu vers la région antérieure, chez les parasites jeunes, au niveau de la touffe d'expansions en baguette. Je n'ai observé ni ornementation cuticulaire, ni fibrilles myocytiques.

Le parasite présente des mouvements à peu près semblables à ceux de $N$. lumbricoides; et ces mouvements sont assez vifs, même chez les exemplaires recouverts par les phagocytes de l'hôte. Ils sont plus amples que chez $N$. lumbricoides et déterminent des ondes qui donnent naissance à une série de renflements et d'étranglements consécutifs donnant au parasite l'aspect que nous avons trouvé aussi chez $N$. magna. 
L'endoplasme, finement granuleux et creusé de petites alvéoles à la périphérie, devient vers le centre grossièrement alvéolaire; il est riche en inclusions chromatoïdes de taille variable, simples granulations parfois, mais parfois aussi masses assez volumineuses.

Les grains de paramylon logés dans les alvéoles sont assez irréguliers comme taille et comme forme.

Le noyau a la même structure que celui de $N$. lumbricoides, mais il renferme un karyosome proportionnellement plus volumineux. Cependant j'ai rencontré certains exemplaires très grands où le karyosome et le noyau étaient excessivement peu développés et semblaient avoir subi une sorte d'atrophie; les parasites qui les contenaient ne présentaient cependant rien d'anormal quant au reste de leur structure (fig. LXVIII $a$ ).

Tous les stades jeunes que j'ai examinés étaient complètement recouverts par les phagocytes de l'hôte. Ces phagocytes sont sur-

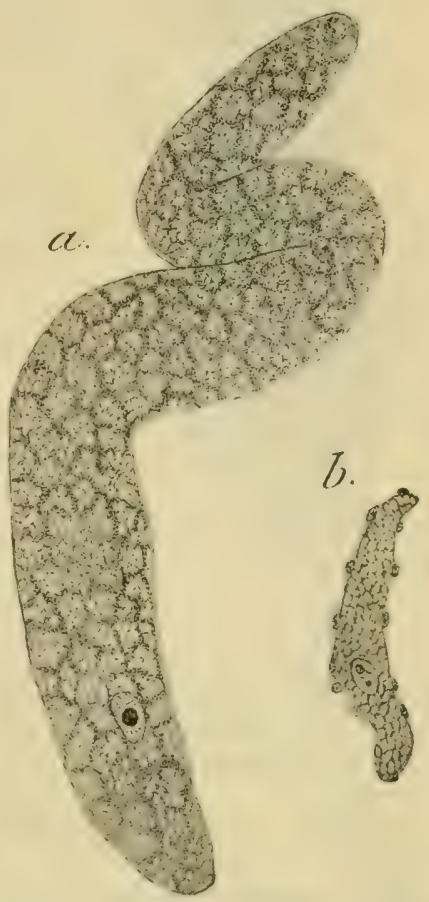

FIG. LXVIII. $N$. vermicularis $\times 120 ; a$. forme libre de phagocytez à noyau très petit; $b$. forme recouverte de phagocytes. tout nombreux et même disposés en plusieurs couches vers les pôles du parasite, où ils s'insinuent entre les prolongements piliformes de la Grégarine (fig. Lxix). Souvent ces deux régions sont comprimées par l'action de ces phagocytes et plus minces que le reste du corps; ceci est surtout fréquent pour la région antérieure, qui constitue alors une sorte de trompe autour de laquelle les phagocytes forment parfois un épais manchon (Fig. Lxx); cette trompe peut même se détacher complètement du reste du corps. 
Lorsque le parasite grandit, les phagocytes sont moins

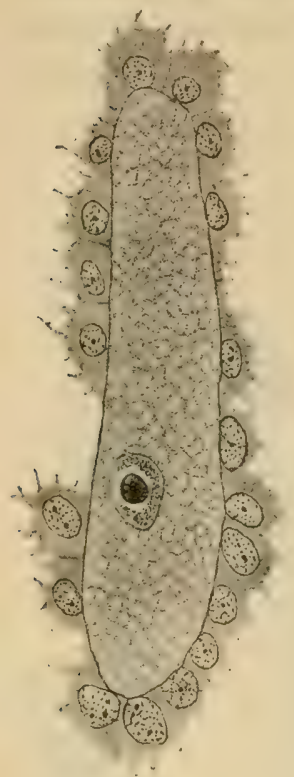

LXIX. serrés et paraissent aussi moins volumineux; on n'en voit plus que quelquesuns épars ça et là à la surface du corps de la Grégarine (fig. LXvIII, b); parfois même ils disparaissent totalement

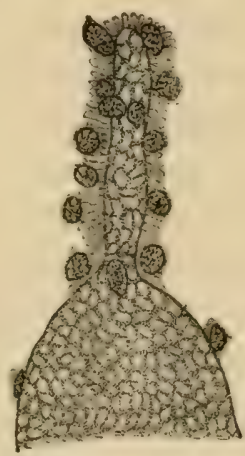

$L X X$.

FIa.LXIX. N. vermicularis. Jeune parasite complètement recouvert de phagocytes $\times 600$. - FIG. LXX. N.vermicularis $\times 600$. Trompe phagocytée.

(fig. LXVIII, $a$ ).

D'après quelques observations faites in-vivo, les individus qui s'accouplent pour l'enkystement s'accoleraient longitudinalement ; malheureusement; je n'ai pu contrôler le fait sur des préparations durables.

Nematocystis anguillula $\mathrm{n}$. sp.

Les vésicules séminales des Pheretima, de même que celles des autres Oligochètes précédemment étudiés donnent asile à la fois à des Grégarines massives et arrondies et à des Grégarines étroites et allongées. Celles-ci ont une forme vermiculaire qui rappelle étonnamment celle des $N$. lumbricoides et $N$. vermicularis des Helodritus; nous les désignerons sous le nom de $N$. anguillula pour marquer leur ressemblance frappante avec un Nématode.

Diagnose. - Corps presque régulièrement cylindrique, nématoïde. Extrémité antérieure terminée en pointe, extrémité postérieure arrondie. Peut atteindre jusqu'à $2 \mathrm{~mm}$. de long sur $40 \mu$ d'épaisseur. Epicyte présentant des stries longitudinales et des 
stries circulaires parallèles. Fibrilles circulaires du myocyte peu visibles, sauf quand le parasite est en état de contraction, Cytoplasme finement réticulaire; grains de paramylon peu volumineux. Noyau ovoïde fusiforme à 2 ou 3 gros karyosomes.

Mouvements très vifs rappelant ceux d'un Nématode.

Kystes fusiformps de $270 \mu$ sur $150 \mu$.

Sporocystes allongés, naviculaires ayant la forme et la structure de ceux des Monocystis du Lombric, et présentant $10 \stackrel{\alpha}{11} \mu$ de grand axe sur $4 \grave{a} 5 \mu$ de petit axe.

Hôtes : Pheretima rodericensis Grube et Ph. hawayana Rosa.

$N$. anguillula est assez rare, elle habite les vésicules séminales de Pheretima rodericensis et de $P h$. hawayana, libre dans le fluide séminal. Comme $R$. pilosa et $R$. porrecta, les parasites sont en général rassemblés par paquets, enroulés, pelotonnés sur eux-mêmes, enchevêtrés les uns dans les autres parmi les éléments sexuels en voie de développement.

$N$. anguillula a la forme d'un petit vermicule; c'est un fuseau très allongé, semblant par suite presque cylindrique et dont une extrémité

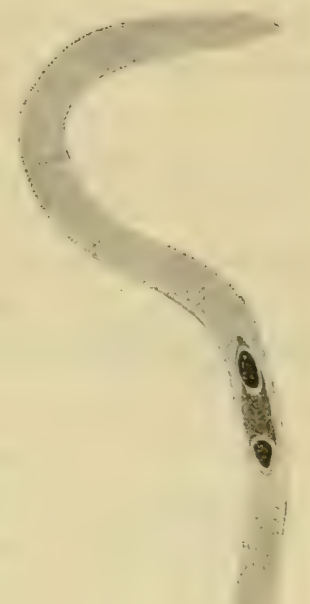
s'amincit légèrement en pointe,

l'autre restant plus épaisse et arrondie (fig. Lxxi). Cette forme est presque identique à celle de $N$. lumbricoides, mais les caractères de l'épicyte et du noyau que nous énumérons 
plus loin permettent de distinguer facilement les deux espèces.

En dehors du va-et-vient habituel des granulations cytoplasmiques, le parasite dont le corps est rigide, non déformable, présente d'assez vifs mouvements de torsion et d'enroulement rappelant ceux d'un petit Nématode. La longueur de $N$. anguillula peut atteindre plus de $2 \mathrm{~mm}$. son épaisseur ne dépasse pas $40 \mu$.

L'épicyte très mince présente des stries longitudinales très nettes visibles sur l'animal vivant sans aucun réactif; et en outre, particularité unique, croyons-nous, chez les Grégarines connues jusqu'ici, il est orné de petits bourrelets circulaires assez espacés, parallèles, à section triangulaire, qui se présentent en coupe optique comme des tubercules latéraux et qui sont aussi marqués que les striations longitudinales, de sorte que le parasite, même observé in-vivo, montre une cuticule ornée d'un quadrillage régulier caractéristique.

La couche de sarcocyte est peu développée, plus épaisse cependant que chez $N$. lumbricoides.

Les fibrilles du myocyte sont fines et peu visibles, on ne les aperçoit avec quelque netteté que dans les points où le parasite s'est contracté. On retrouve ici le phénomène que nous avons signalé chez $N$. lumbricoides; là où le parasite s'infléchit, se recourbe sur lui-même, il se forme des plis très accentués de l'ectoplasme, plissements en accordéon dont l'aspect rappelle celui des plissements longitudinaux décrits par Porter (1897) chez une Grégarine intestinale de Rhyncobolus americanus Verril.

L'endoplasme finement granuleux et alvéolaire renferme dans ses mailles des grains de paramylon peu développés. Il contient en outre assez fréquemment de grosses masses chromidiales dont la structure est voisine de celle du karyosome.

Le noyau n'a pas de situation déterminée; en général logé vers le milieu du corps, il peut aussi se rapprocher de l'une quelconque des deux extrémités. Il est ovoïde ou naviculaire et renferme presque constamment 2 gros karyosomes situés 
aux deux extrémités du grand axe ; parfois, un troisième karyosome plus petit est logé au centre du noyau (fig. LxxiI). Il peut n'y avoir qu'un seul karyosome comme chez $N$. lumbricoiles, mais ce fait est très rare. Chacun de ces karyosomes est entouré par une zone claire qui le sépare du nucléoplasma. Ces zones sont traversées par les grêles filaments du réseau de linine qui semblent s'insérer sur les karyosomes et rayonner de là dans le reste du noyau. De nombreuses granulations chromatiques sont disposées sur ce réseau. Le tout baigne dans un suc nucléaire fortement colorable. Sur les frottis colorés, l'une-des extrémités du noyau présente souvent un prolongement étiré se terminant par un bouton dans lequel le karyosome voisin envoie un prolongement renflé en boule à son extrémité. Je n'ai malheureusement pas observé ces faits sur le vivant et ne puis me prononcer sur leur signification, ce n'est peut-être là qu'une simple déformation due à une fixation défectueuse, mais peut-être s'agit-il d'un véritable prolongement amœboïde du noyau.

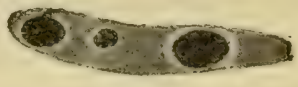

$L X X I I$.

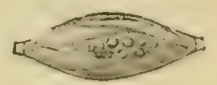

$L X X I I I$.

FIG. IXXII. Noyau de $N$. anguillula $\times 200$. FIG. LXXIII. Sporocyste de $N$. anguillula $\times 1.300$.

Les kystes sont fusiformes et atteignent en moyenne $270 \mu$ sur $150 \mu$. Ils contiennent des sporocystes biconiques, naviculaires, allongés, ayant tout à fait la forme et la structure de ceux des Monocystis du Lombric et mesurant 10 à 11 p. de grand axe sur 4 à $5 \mu$ de petit axe ; ils renferment 8 sporozoïtes dont la disposition est la même que chez les espèces précédemment étudiées (fig. LXxIII).

Nous n'avons pas suivi le développement de cette espèce et nous n'avons jamais eu l'occasion d'observer des stades très jeunes.

\section{Stomatophora coronata Hesse.}

Nous avons décrit, dans une communication au Congrès de Grenoble de l'Association française pour l'avancement 
des Sciences (août 1904), une Grégarine parasite de divers Pheretima et à laquelle nous avons donné le nom de Monocystis coronata. Une courte diagnose différentielle de ce parasite a paru dans le Bulletin trimestriel de l'A.F.A.S. (novembre 1904, $\mathrm{n}^{\circ} 9$ p. 268).

Depuis, Drzewecrr (1907), se basant sur la présence d'une bouche? véritable et d'un anus? ? a créé pour cette espèce un genre nouveau, le genre Stomatophora. Bien que nous ne soyons pas d'accord avec l'auteur sur la valeur de la " bouche » du parasite et que nous n'ayons pu nous convaincre de la présence de l'anus malgré de nombreuses et minutieuses recherches, nous pensons qu'il est bon de maintenir le genre Stomatophora pour la Grégarine à ventouse des Pheretima, à cause des particularités de structure de ce parasite, notamment à cause de sa ventouse. Voici une diagnose du parasite un peu plus complète que celle que nous avons donnée antérieurement :

Monocystidée à corps sphérique, ovoïde ou ellipsoïdal présentant jusqu'à $180 \mu$ sur $130 \mu$. A la partie antérieure, il existe une sorte de ventouse radiée formée d'une cavité sphérique présentant la forme du moulage en creux d'un melon à côtes saillantes; le plafond de cette cavité, constitué uniquement par de l'ectoplasme hyalin, a la forme d'une couronne pétaloüde. Epicyte très mince, orné de stries méridiennes. Cytoplasme finement granuleux renfermant des grains de paramylon ovoüdes assez volumineux. Mouvements en sablier analogues à ceux de M. lumbrici, mais un peu plus lents.

Kystes ellipsoüdaux ou fusiformes ayant en moyenne 70 à $80 \mu$ sur 50 à $60 \mu$. Sporocystes naviculaires ayant la même structure que ceux des Monocystis du Lombric, mais à extrémités moins pointues, élargies, présentant une sorte de bouton aplati à l'aide duquel les sporocystes se soudent de façon à former de longues chaînes dans l'intérieur des kystes.

Il y a des macrosporocystes et des microsporocystes : les dimensions extrêmes de ces spores sont $11 \mu$ sur $6 \mu$ et $7 \mu$ sur $3 j$. Hôtes : Pheretimarodericensis Grube et Ph. hawayana Rosa. 
Stomatophora coronata est très abondante dans les vésicules séminales de deux Oligochètes exotiques acclimatés dans les serres du Jardin des Plantes de Grenoble: Pheretima rodericensis Grube et Pheretima hawayana Rosa.

Le parasite se développe dans les blastophores dont il épouse la forme sphérique. Il garde souvent cette forme, même lors-

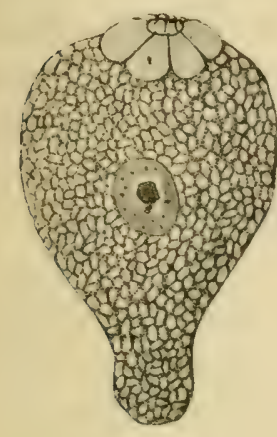

$L X X I V$

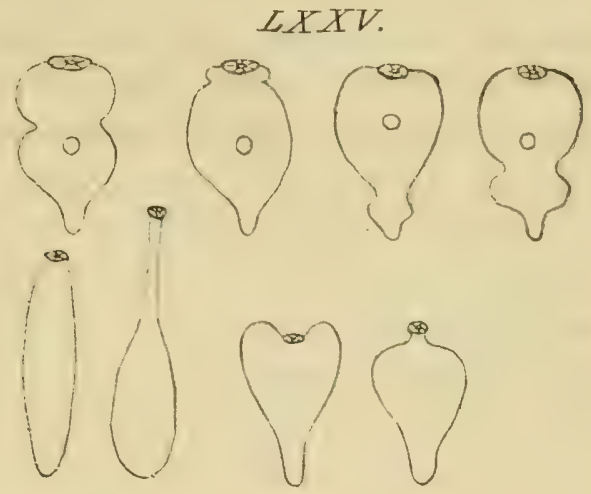

L.Y.TH\%

FIG. LXXIV. St. coronata sortie du blastophore nourricier $\times 650$. - FIG. LXXV. Divers aspects de St, coronata en mouvement. Schéma. - FIG. LXXVI. Formes diverses de $S t$. coronata. Schéma.

qu'il devient libre dans les vésicules séminales; mais il peut devenir aussi ovoïde ou ellipsoïdal. L'axe d'allongement est antéro-postérieur, il passe par la ventouse, appareil épiméritique caractéristique de l'espèce et situé au pôle antérieur (fig. LXXIV).

La longueur peut atteindre jusqu'à $180 \mu$, l'épaisseur jusqu'à $130 \mu$.

St. coronata est très agile; ses mouvements rappellent tout à fait ceux de $M$. lumbrici Henle quoique un peu moins rapides; ce sont des mouvements en sablier auxquels participent les grains de paramylon et le noyau lui-même. Ces mouvements produisent dans le corps de la Grégarine des changements de forme importants que l'on peut observer dans les frottis colorés, et que nous avons trouvés déjà chez $M$. lumbrici; 
ces changements sont représentés (fig. LXV et LXVI) où l'on voit des parasites en fuseau asymétrique, en massue, en cœur, en toupie, etc. Dans tous ces cas, la ventouse est toujours placée à l'extrémité opposée à la pointe, elle est située à l'extrémité antérieure par conséquent.

Vue de face, cette ventouse a l'aspect d'un cercle de faible diamètre entouré par une collerette pétaloïde claire. Les pétales de la collerette sont ornés d'une série de fines stries radiales qui sont des stries épicytaires (fig. LxxviI). Au centre du cercle, on aperçoit un point sombre d'où s'échappent
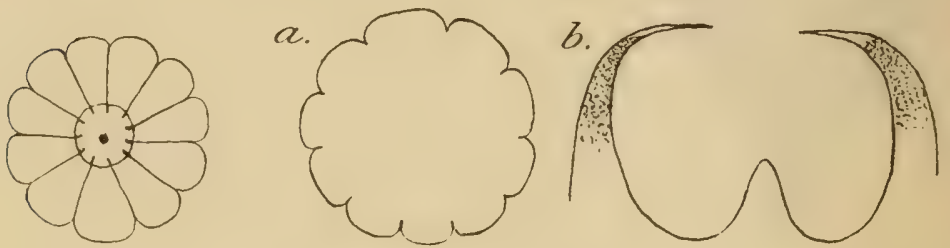

$L X X V I I$.

\section{LXXVIII.}

Fig. LXXVII. Ventouse de St. coronata Schéma. - FrG. IXXVIII. Deux coupes perpendiculaires de la ventouse de St. coronata. Schéma; $a$. coupe perpendiculaire au grand axe du parasite; $b$, coupe passant par l'axe du parasite.

des rayons colorés qui se continuent par les lignes de séparation des divers pétales. Ces lignes paraissent se prolonger au dedans du cercle en formant de petites denticulations radiales.

L'examen de frottis et de coupes sériées permet de comprendre la constitution de cet appareil. Schématiquement, c'est une sphère creuse de laquelle on aurait détaché une calotte polaire, l'ouverture ainsi pratiquée étant l'orifice de la ventouse, la bouche du parasite pour Drzewechi. La figure LxxviII donne deux coupes perpendiculaires de cette cavité.

Les parois de la ventouse ne sont pas lisses, elles présentent une série de côtes méridiennes faisant saillie dans la cavité. Les arêtes de ces côtes ainsi que le rebord circulaire de l'ouverture se colorent avec intensité par le Fer. Une comparaison banale nous permettra de mieux expliquer la forme de l'appareil, c'est 
assez exactement le moulage en creux d'un melon à côtes saillantes.

La cavité n'est que rarement sphérique; le plus souvent, son plancher est soulevé à la façon d'un fond de bouteille et le pôle d'où partent les côtes méridiennes se trouve rapproché de l'ouverture de l'appareil (fig. Lxxvin, b) c'est ce pôle qui apparaît comme un point sombre au centre du cercle à bords colorés. On peut le considérer comme homologue du mucron conique de $M$. lumbrici.

Le plafond peut être formé uniquement par l'épicyte replié sur lui-même et entre les deux replis duquel il y a une légère couche de sarcocyte hyalin; c'est le cas le plus commun.

L'ensemble prend alors l'aspect d'une collerette claire que nous avons signalée. Une certaine quantité d'endoplasme peut pénétrer en même temps que le sarcocyte entre les deux replis épicytaires. La ventouse vue de face n'offre plus alors de collerette, elle est réduite au cercle d'ouverture avec ses denticulations et au point sombre central avec les rayons colorés qui en partent et qui semblent s'arrêter au pourtour de l'orifice.

Cette forme fondamentale de la ventouse se modifie plus ou moins profondément suivant l'état de contraction et suivant ]'âge du parasite. L'ouverture peut être portée sur un cylindre court à paroi présentant des stries longitudinales; ce cylindre s'allongeant considérablement en diminuant son diamètre, il devient un long pédicule strié comme le représente la figure 45. Le cercle d'ouverture, d'un diamètre ordinairement réduit, peut s'agrandir beaucoup par suite d'une sorte d'extroversion du plafond (fig. 47) et l'on arrive à avoir au lieu d'une ventouse un plateau strié, à bords ondulés (fig. 48). Ce plateau montre parfois des bords très déchiquetés (fig. 46) ; les différents lobes se déchiquetant peu à peu, puis se détachant, on a là un processus qui amène la disparition de la ventouse ; certaines Grégarines n'ont plus que un ou deux lobes du plateau, d'autres n'offrent plus trace de ventouse ni de plateau. Cette disparition de la ventouse n'a lieu que chez les Grégarines libres ; dans l'inté- 
rieur du blastophore, le parasite possède toujours une ventouse, celle-ci persiste même quelquefois assez longtemps chez les formes libres, le plus souvent cependant, elle s'atrophie rapidement.

L'épimérite est done ici parfois transitoire, ce fait le rapproche de celui de N. magna et de celui des Polycystidées. On pourrait donc être tenté de considérer $S t$. coronata comme une Dicystidée voisine des Schneideria par exemple, mais l'étude du développement ne permet pas d'adopter ce rapprochement ; du reste l'épimérite n'est pas toujours caduc, très souvent l'accouplement a lieu entre Grégarines encore pourvues de leur ventouse, c'est-à-dire entre céphalins, ce qui n'a jamais lieu chez les Polycystidées.

C'est sans doute l'orifice de la ventouse que Drzewecki considère comme la bouche du parasite, ses dessins sont trop schématiques pour qu'on puisse l'affirmer, il ne paraît pas avoir vu la collerette, cependant il la représente très grossièrement (fig. 54, taf. 9), sans en indiquer la véritable nature et il parle aussi d'un péristome nettement séparé du reste du corps. Il déclare que la bouche ne joue pas toujours un rôle important dans la nutrition du parasite, l'absorption des aliments solides pouvant se faire en un point quelconque du corps. Pour notre part nous n'avons pas vu le parasite absorber d'aliments solides, pas plus par la bouche que par le reste de la surface du corps et la ventouse nous paraît tout à fait comparable comme structure et comme fonction aux divers épimérites que nous avons signalés chez d'autres Monocystidées. Sa complexité rappelle celle de la trompe de $R$. pilosa, mais avec une forme toute différente, le mucron étant ici presque rudimentaire tandis que la couronne de sarcocyte hyalin est très développée. Nous avons vu que l'épimérite de $R$. pilosa servait non seulement à la fixation du parasite, mais encore qu'il jouait certainement un rôle dans la nutrition. Il en est sans doute de même pour la ventouse de St. coronata qui fonctionnerait comme un véritable suçoir fixant le parasite au corps de l'hôte et s'emplis- 
sant de substance nutritive qui pénètre ensuite par osmose dans l'intérieur du parasite.

Lorsque St. coronata est encore contenue dans l'intérieur $\mathrm{du}$ blastophore, on peut voir les noyaux altérés des cellules sexuelles rassemblés en face de l'ouverture de la ventouse comme s'ils avaient été aspirés, ils ne pénètrent cependant pas dans la cavité de la ventouse. Certains parasites recouverts par des cellules sexuelles altérées ayant la forme de larges lanières cytoplasmiques qui leur constituent une épaisse toison, laissent très bien voir le jeu de la ventouse ; la figure 143 représente la coupe d'une Grégarine suspendue par cette ventouse à la paroi interne de la cavité qu'elle s'est creusée dans le blastophore ; le cytoplasme blastophorien remplit cette cavité ; parfois, on voit aussi y pénétrer la base des cellules sexuelles supportées par le blastophore et situées en face de l'orifice. Lorsque le parasite rompt l'enveloppe blastophorienne devenue trop étroite pour le contenir, il peut rester encore quelque temps avec la cavité de sa ventouse pleine des restes de la cellule-hôte et des cellules sexuelles que supportait cette cellule. Nous n'avons jamais vu ni ces cellules, ni leur noyau incorporés ensuite directement dans le cytoplasme du parasite. La nutrition de Stomatophora coronata, de même que celle de toutes les autres Grégarines se fait toujours par osmose.

Nous avons recherché en vain l'anus, signalé par DrzeWECKI; est-ce parce que nous n'avons pas eu la chance de fixer une seule Grégarine au moment de la défécation? Ou ne serait-ce pas plutôt parce que le parasite se nourrissant uniquement par osmose n'a pas besoin d'orifice pour rejeter des déchets excrémentitiels solides?

L'ectoplasme est très réduit, il se compose d'un mince épicyte et d'une faible couche de sarcocyte. Ces formations ne sont mises en évidence avec quelque netteté que par le Mann qui les colore en bleu. Nous avons vu que l'ectoplasme constituait souvent à lui seul le plafond de la ventouse et formait la collerette colorée en bleu par le Mann (fig. 187) et qui 
reste incolore avec les autres teintures. Les ornements de la cuticule sont très nettement visibles dans cette région, ce sont des stries de grandeur variable, les unes très accentuées séparent les divers pétales, les autres, plus fines, assez serrées se voient sur ces pétales eux-mêmes; ces diverses stries se prolongent sur le reste du corps (fig. 40), mais il est très difficile de les apercevoir avec quelque netteté. Lorsque le parasite se contracte en s'étirant, ces ornements épicytaires sont bien mis en évidence; dans les formes en massue par exemple, le manche paraît très nettement strié. C'est également sur ces constrictions que l'on peut apercevoir les fines fibrilles transversales parallèles du myocyte qui sont invisibles partout ailleurs.

L'entocyte est constitué par un cytoplasme alvéolaire dans lequel on observe diverses inclusions : grains de réserve et masses chromatoïdes.

Les travées cytoplasmiques sont formées par un amas de fines granulations dans lesquelles sont noyés quelques grains plus volumineux et diverses masses chromidiales. Dans les alvéoles on aperçoit les grains de paramylon ellipsoïdes ou sphériques, de taille variable, le plus souvent très nombreux et très serrés, de sorte que les travées cytoplasmiques qui les séparent sont excessivement étroites. Leur structure est absolument celle que nous avons indiquée en décrivant $M$. lumbrici; cavité centrale sphérique ou irrégulière paraissant rouge vue au microscope in vivo et se colorant avec intensité par le Rouge Magenta et la Safranine, série de zones concentriques demeurant généralement incolores, mais se colorant cependant avec intensité par le Violet de Gentiane.

Comme chez $M$. lumbrici, le noyau n'a pas de situation fixe il est chassé d'une extrémité du corps à l'autre par les contractions du parasite et suit dans leurs mouvements les grains de paramylon et les granulations cytoplasmiques. Dans le parasite au repos, il occupe le centre du corps. C'est une sphère entourée par une membrane qui retient avec intensité les colo- 
rants chromatiques. Il renferme un karyosome sphérique vacuolaire plus ou moins fortement coloré, avec un petit grain très foncé presque constamment accolé à sa surface, ce grain correspond sans doute au "karyosome " décrit par LÉGER (1904 a) chez Stylorhynchus. Des grains chromatiques sont disposés sur un cercle qui entoure le karyosome, séparés de lui par une zone claire, ou dispersés dans tout le noyau sur un réseau de linine très délicat; souvent, un certain nombre de ces grains sont accolés à la membrane nucléaire.

Le karyosome peut être central ou excentrique; dans ce dernier cas, les granulations chromatiques au lieu d'être disposées régulièrement autour de lui se rassemblent à l'un des pôles en un champ ayant la forme d'un croissant. Très rarement, on observe deux karyosomes.

Nous verrons en étudiant le développement que ce karyosome change souvent d'aspect et de colorabilité au cours de la vie du parasite.

La reproduction a lieu uniquement par voie sexuée comme chez les Monocystis du Lombric. Je n'ai pas observé d'enkystements solitaires comme ceux qui ont été signalés souvent chez les Monocystis et que Cecconi (1902) considère comme conduisant à la sporulation chez un $M$. agilis parasite de Allobophora complanata. Tous les kystes que j'ai rencontrés renfermaient deux individus. L'accolement se fait toujours par la ventouse, ou,lorsque celle-ci a disparu,par le pôle qu'elle occupait, c'està-dire, le pôle antérieur. Le mouvement en sablier continue pendant un temps assez long, sa vitesse semble même s'accélérer au début, puis l'immobilité survient et la paroi kystique est sécrétée.

Les dimensions et la forme des kystes varient considérablement; ils sont ellipsoïdaux ou sphériques, parfois ce sont des fuseaux fortement renflés à l'équateur; dans ce dernier cas, la paroi kystique a été sécrétée avant que les parasites copulant se soient contractés (fig. LxxIx); le plus souvent, avant de sécréter cette paroi, ils replient leur région caudale sur le 
reste du corps de façon à prendre une forme arrondie, ainsi se constituent les kystes ellipsoïdaux (fig. Lxxx) ou sphériques. La membrane une fois secrétée, les Grégarines continuent encore le plus souvent à se contracter de sorte qu'il existe un large espace vide entre elles et la paroi du copularium, espace qui est encore augmenté par l'action des fixateurs; ce fait a été remarqué également chez les Grégarines du Lombric par

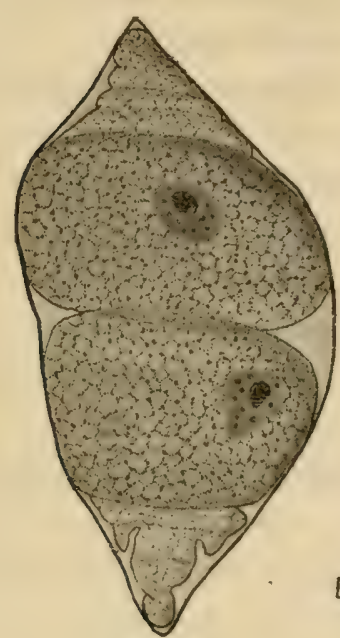

$L X X I X$ :

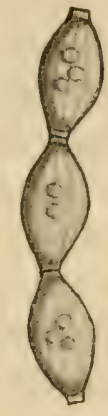

$L X X X I I$

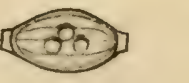

L. $X X X$ T.

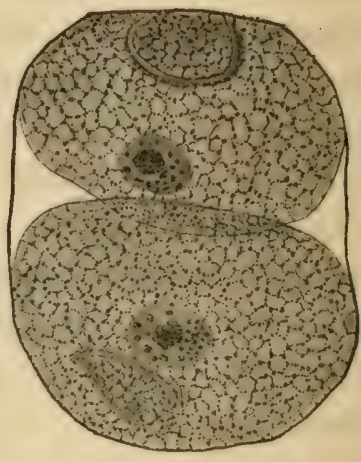

LXYX.

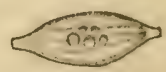

LXXXIII.

FIG. LXXIX, St, coronata $\times 650$. Kyste fusiforme, - FIG. LXXX. St, coronote $\times 650$. Kyste ellipsoïdal. - FIG. LXXXI. Sporocyste de St. coronota $\times 1300$. - FIG. LXXXII. Chaîne de sporocystes de St. coronata $\times$ 1.300. - FiG. LXXXIII. Sporocyste de St. diadema $\times \mathbf{1 . 3 0 0 .}$

divers observateurs. Les parasites enkystés se meuvent parfois encore quelque temps de sorte que le plan d'accolement se déplace. Ordinairement perpendiculaire au grand axe du kyste, ce plan passe parfois par cet axe même; les deux conjoints ont donc opéré une rotation de $90^{\circ}$ par rapport à leur disposition primitive.

Les kystes ont en moyenne 60 à 70 u. de diamètre ou 75 à $85 \mu$ sur 50 à $60 \mu$.

L'accouplement peut avoir lieu entre deux parasites encore 
renfermés dans les blastophores; les kystes sont alors recouverts par les restes du blastophore : une membrane mince supportant les spermatozoïdes flétris que nous étudierons plus loin (fig. 156). NASSE (1882) a signalé un fait analogue pour les kystes de Urospora tubificis chez les Tubifex.

Je n'ai pas suivi les processus de la fécondation qui rappellent beaucoup ceux que BrasiL (1905b) a signalés chez les Monocystis de Lumbricus herculeus Sav. Il semble y avoir ici une légère différenciation sexuelle entre les conjoints; dans l'un, on observe en effet, au moment de la multiplication nucléaire conduisant à la formation des gamètes, des fuseaux assez courts, fortement renflés à l'équateur; dans l'autre les fuseaux sont au contraire plus allongés et plus grêles. Les mitoses ne sont pas synchroniques, elles se font plus rapidement chez l'un des individus. Il y a également anisogamie, mais anisogamie peu marquée : on observe des gamètes un peu allongés à noyau condensé et des gamètes arrondis à noyau dilaté. Ces gamètes copulent deux à deux pour donner une copula arrondie qui se transforme en sporocyste.

Les sporocystes ont une forme intermédiaire entre la forme en fuseau pointu aux deux extrémités des Monocystis des Oligochètes et la forme en tonnelet des Gregarina. Au lieu de se terminer en pointes, ils présentent à leurs extrémités un bouton élargi (fig. LxxxI), à l'aide duquel ils se soudent les uns aux autres de façon à constituer dans l'intérieur du kyste de longs chapelets contournés (fig. LXXxir).

Ces sporocystes ont des dimensions constantes dans un même kyste, mais ces dimensions varient avec les kystes considérés. Il y a des macrosporocystes de 9 à $10 \mu .5$ de long sur 5 à $6 \mu$ de large et des microsporocystes de $7 \mu$ à $7 \mu$ sur $3 \mu$. Il existe du reste des sporocystes à dimensions intermédiaires entre ces extrêmes.

Tous ces sporocystes renferment 8 sporozoïtes disposés comme dans les sporocystes des autres Monocystidées et présentant un noyau central ou situé au toisinag? de l'une des extré- 
mités, ils entourent un reliquat central à 3 gros grains réfringents.

Ces sporocystes occupent la périphérie du kyste. Le centre est rempli par un reliquat abondant dans lequel s'observent d'assez nombreux noyaux, soit noyaux gamétiques non arrivés à maturation, soit noyaux somatiques; ceux-ci contiennent un suc nucléaire clair et abondant, et toute leur chromatine est accolée à la membrane.

Développement. - De même que celle des Monocystis du Lombric, la spore de St. coronata s'ouvre dans l'intestin de l'hôte sous l'action des sucs digestifs, sa déhiscence est également apicale; les sporozoïtes sortent par les deux pointes ainsi ouvertes et nagent dans le liquide intestinal. Je n'ai pu suivre leur migration et ne sais comment ils arrivent dans les vésicules séminales.

Les premiers stades que j'ai observés étaient déjà dans les follicules spermatiques ou en train d'y pénétrer. Cette pénétration peut avoir lieu à un stade quelconque du développement de ce follicule, depuis l'instant où il se détache du testicule pour tomber dans les vésicules séminales où il doit achever son évolution, jusqu'au moment où, cette évolution achevée, le blastophore se trouve dépouillé des cellules sexuelles qu'il supportait (fig. 43).

Lorsque le blastophore est formé, la jeune Grégarine pénètre directement dans son intérieur;avant la constitution du blastophore, elle se loge dans le pédicule de l'une des callules sexuelles, au voisinage du centre du follicule; elle se trouve ainsi dans l'intérieur du blastophore lorsque celui-ci se constitue par un processus que nous avons indiqué dans un récent travail (1909). Nous avons obsərvé des parasites on voie de pénétration et dont une partic du corps était encore en dehors de la cellule hôte; ils ont en ce moment la forme d'un fuseau asymétrique, une des extrémités pointues est en avant, l'autre arrondie se trouve en arrière (fig. 137). Drzewecki décrit et figure des sporozoïtes arrondis à noyau terminal qu'il a trouvés 
nageant dans les vésicules séminales; nous n'avons pu retrouver de tels sporozoïtes libres arrondis, leur existence nous parait problématique, car avec une telle forme il leur serait bien difficile de pénétrer dans les blastophores de l'hôte, Drzewecki ne nous renseigne pas sur leur mode de pénétration; du reste il déclare lui-même avoir combiné le cycle évolutif du parasite: d'après ses observations sur les Monocystidées du Lombric, sur Lankesteria ascidiae de Ciona intestinalis et aussi de Stomatophora coronata; cette méthode l'a conduit à des résultatis par trop fantaisistes.

Les dimensions des jeunes parasites observés en voie de pénétration sont variables :

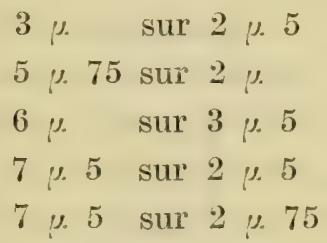

Quelle que soit leur taille, leur structure ne varie guère. Ils présentent une zone eatoplasmique très nette colozéc. en gris bleu par le Mann et un endoplasme finement granuleux très dense. Le noyau logé dans la partie médiane renfléc dı fuscau est entouré d'uno mombrane peu distincte, achromatique; il renferme un karyosome assez voluminoux, cxcentrique, entouré par une large zone claire, et des grains chromatiques peu nombreux. Dans les parasites très petits, ces grains sont accolés à la membrane (fig. 136), c'est le "Kernnucleolus " de Drzewecki; dans les exemplaires plus volumineux, ils sont disposés on un cercle régulier autour de la zone claire périkaryosomienine, et une partie seulement d'entr'eix sont placés sur la membrane, au point où le karyosome est voisin de cette membrane; le reste du noyau qui se présente comme un croissant en coupe optique est rempli de grains chromatiques plus petits; c'est le "Nucleolenring " de Drzewnecki (fig. 42, 43, 44, 137, 138). 
Un grain fortement coloré est visible dans le cytoplasme, parfois tangent au noyau (fig. 137), mais le plus souvent situé à une assez grande distance de celui-ci au voisinage de la pointe (fig. 44); je pense que ce grain correspond à celui que LÉGER et Duboscq (1902, 1904) ont décrit dans les sporozoïtes de Stylorhynchus longicollis F. Stein et de Clepsidrina Munieri et qu'ils considèrent comme un centrosome. Les mêmes observateurs signalent chez certains sporozoïtes de Stylorhynchus " une mince traînée linéaire légèrement teintée " qui "relie le centrosome à la base du rostre ". Nous avons vu quelquefois ici le centrosome relié au noyau par une ligne faiblement colorée (fig. 138) ; mais cette ligne ne se prolonge pas au delà du centrosome. Au point où elle touche la membrane nucléaire, on observe parfois un épaississement punctiforme de cette membrane qui peut être relié au karyosome par un filament délié. On peut voir aussi quelquefois une ligne ondulée partant du noyau et aboutissant à l'extrémité du rostre ; tantôt cette ligne est incolore et présente l'aspect d'un canal clair (fig. 138), tantôt, elle se teinte assez fortement par le Fer (fig. 42); nous l'avons observée concurremmentavec la ligne colorée qui joint le centrosome à la membrane nucléaire (fig. 42). C'est une formation tout à fait comparable à celles qui ont été décrites par LÉGer (1901) chez Aggregata colomica et par LÉG ER et DuboscQ (1903) chez Stenophora Brölemanni. Elle fait penser, à cause de sa situation et de sa forme à une sorte de canal digestif rudimentaire, mais il est bien difficile de se prononcer sur sa véritable nature. Je ne l'ai pas retrouvée chez le parasite adulte et j'ignore qu'elle est sa destinée.

L'extrémité arrondie du parasite présente une petite masse de cytoplasme différencié, hyalin in vivo, et qui se colore en vert intense par le Vert lumière, comme le mucron des 1.onocystis du Lombric et comme le petit mamelon qui occupe le fond de la ventouse chez les $S t$. coronata adultes (fig. 70, 137). D'autres fois on observe dans cette même extrémité une volumineuse vacuole présentant un grain sidérophile, 
soit accolé à sa surface (fig. 43,44, 70), soit contenu dans son intérieur. La figure 55 représente un tel grain relié au karyosome par un filament et rappelant l'aspect des centrosomes précédemment décrits. Il s'agit pent-être là du début de la formation de la ventouse, mais je ne suis pas en mesure de l'affirmer, car je n'ai pu suivre ses transformations jusqu'au moment de l'apparition de cette ventouse. C'e serait en tous cas un fait bien curieux de voir ainsi l'extrémité postérieure du sporozoïte devenir l'extrémité antérieure de l'adulte.

Lorsqu'il s'est installé dans la cellule hôte, le parasite grossit, son petit axe s'accroît beaucoup plus vite que le grand axe (fig. 55, 70) et la Grégarine peut devenir complètement sphérique; j'en ai remarqué de cette forme qui n'avaient pas plus de $3 \mu 5,4 \mu 5$ ou $6 \mu$ de diamètre.

Le parasite qui a achevé sa croissance peut être sphérique, ellipsoïdal ou ovoïde, généralement son grand axe est antéropostérieur c'est-à-dire qu'il passe par l'axe de la ventouse, mais il arrive assez souvent qu'il est perpendiculaire à cet axe.

Les dimensions proportionnelles du parasite, du noyau et du karyosome sont à peu près les mêmes que celles que nous avons relatées pour $M$. agilis, ainsi qu'on peut le voir en comparant le tableau suivant avec celui que nous avons donné pour cette dernière espèce :

\begin{tabular}{|c|c|c|c|}
\hline \multicolumn{2}{|r|}{ Corps du parasite } & Noyaux & Karyosomes \\
\hline $3 p$ & $\mu . \quad$ sur $\quad 2 \mu .5$ & $1 \mu .5$ & $0 . \mu .5$ \\
\hline 5 & $\mu \quad \operatorname{sur} 3 \mu$ & $2 \mu$ & $0 \mu 5$ \\
\hline & $\mu . \quad$ sur $4 \mu .5$ & $2 \mu .5$ & $1 \mu$. \\
\hline & p. de diamètre & $3 \mu$ & 1 «. 5 \\
\hline & u. 5 & $4 \mu .5$ & $2 \mu$ \\
\hline 10 & $\mu$ & $5 \mu$ & $2 \mu 75$ \\
\hline 15 & 5 & $5 \mathrm{u}$ & $3 \mu$ \\
\hline 19 & «. 5 & $6 \mu$ & $3 \mu$ \\
\hline 30 & $u \quad \operatorname{sur} 25 \mu .5$ & $7 \mu .5$ & $3 \mu$ \\
\hline 48 & $\mu . \quad \operatorname{sur} 31 \mu .5$ & $9 \mu \quad$ sur $7 \mu 5$ & $3 \mu .5$ \\
\hline 54 & $\mu \quad$ sur $29 \mu_{0}$ & $9 \mu$ & $3 \mu$ \\
\hline
\end{tabular}


La Grégarine emplit complètement le blastophore dont la paroi s'applique intimement sur sa surface (fig. 139, 140, 141, $142,187)$, puis elle le distend parfois jusqu'à le briser ; elle s'échappe alors de son enveloppe et se trouve libre dans les vésicules séminales; là elle peut continuer à s'accroître et atteindire jusqu'à 180 u. de long sur 130 de large, mais c'est une exception, les dimensions ne dépassent pas en moyenne 80 y. sur 60 y.

St. coronata ne quitte pas toujours son enveloppe blastophorienne, nous avons vu que l'accouplement et l'enkystement peuvent se produire alors queles parasites sont encore contenus dans les blastophores nourriciers réduits à leur paroi et à leur recouvirement de cellules sexuelles altérées.

Il n'y a le plus souvent qu'une seule Grégarine par blastophore; mais on en observe quelquefois 2 (fig. 73) et même 3 qui ont envahi le même follicule et s'y développent concurremment. On pourrait croire que les kystes revêtus de spermatozoïdes altérés ont pour origine des accouplements entre des parasites cohabitant dans le même cytophore; c'est sans doute le cas pour quelques-uns, mais il en est d'autres qui proviennent manifestement d'accouplements entre parasites de blastophores différents ainsi que nous avons pu l'observer in vivo.

Pendant que le parasite s'accroit, son cytoplasme change d'aspect. Dès que la Grégarine s'arrondit, le canal clair ou coloré qui allait du noyau à la pointe antérieure cesse d'être visible; par contre, on continue à apercevoir à un pôle une région différenciée colorée en vert par le Vert lumière et à laquelle est souvent accolé un grain chromatoïde. D'autres granulations chromatoïdes apparaissent dans le plasma et s'y multiplient; quelques-unes sont peu volumineuses, ce sont de petits grains ou de petits bâtonnets irréguliers; mais la plupart sont beaucoup plus grandes, elles sont formées de deux sphères une grande et une petite, soit tangentes, soit un peu éloignées et reliées par un filament (fig. 69). C'est là une forme caractéristique de St. coronata, comme les chromidies en fer de lance sont caractéristiques de $M$. lumbrici. Ces grosses masses sont fortement 
sidérophiles, elles sont souvent entourées d'une vacuole. Elles s'émiettent dans le cytoplasme et l'on voit ensuite apparaître les grains de réserve, à chacun desquels est accolé au début un petit grain chromatoïde. Lorsque la réserve de paramylon est complètement développée, les granulations chromatoïdes disparaissent.

Le Mann colore en rouge des formations qui semblent bien être les mêmes (fig. 192). De plus il met en évidence uno grande quantité de fines granulations rouges (fig. 193) qui forment un amas allant de la ventouse au noyau et qui représentent peutêtre un premier stade de la transformation des matières nutritives absorbées; il y aurait ainsi une sorte de courant nutritif entre la ventouse et le noyau.

Lorsque les grains de paramylon sont bien développés, ils sont étroitement serrés les uns contre les autres et il ne persiste entr'eux que d'étroites zones d'un cytoplasme finement granuleux.

C'est dans ce cytoplasme, que l'on voit reparaître, chez les parasites adultes des grains chromatoïdes plus gros que les autres granulations cytoplasmiques, mais toujours considérablement moins grands que les masses chromidiales des parasites en voie d'accroissement; des gouttelettes se forment ensuite que le Mann colore en rouge pâle et qui semblent contenues dans des vacuoles; d'abord petites, elles grandissent en même temps que la vacuole augmente et que les grains de paramylon disparaissent (fig. 188, 190).

Bientôt la colorabilité du cytoplasme change totalement: il était éosinophile, il offre maintenant une grande affinité pour l'Orange. Le Mann lui donnait une coloration bleu pâle, ce même colorant le teinte en bleu foncé, puis en violet. Dans les kystes où les sporoblastes ont copulé et évoluent en sporocystes, la coloration du cytoplasme de reliquat change encore, il prend une teinte rouge par le Mann. Ces changements de colorabilité sont dus sans doute à la dissolution dans le cytoplasme des gouttelettes rouge pâle mises en évidence par le Namn et. 
qui, souvent très nombreuses au moment de l'enkystement des parasites ne tardent pas à disparaître par la suite à mesure que la tonalité rouge du cytoplasme augmente. Quant à ces gouttelettes elles-mêmes, elles proviennent probablement de la transformation des grains de réserve et ne sont peut-être qu'un stade de leur assimilation. Nous avons émis déjà cette hypothèse à propos de $M$. agilis et nous verrons en étudiant les phénomènes de dégénérescence observés chez diverses Monocystidées qu'une telle hypothèse n'est pas dénuée de fondement. De même que chez Monocystis agilis, les grains chromatoïdes dont le cytoplasme est parsemé à ce moment et qui sont d'origine nucléaire jouent sans doute un rôle primordial dans cette assimilation.

Le " noyau flammé » n'existe pas ici, je ne l'ai jamais observé du moins, mais s'il n'y a pas de prolongements nucléaires amœboïdes par lesquels les granulations chromatiques s'écoulent dans le cytoplasme, la membrane nucléaire semble parfois disparaître, le noyau n'étant plus limité que par les grains de paramylon placés en bordure et les grains de chromatine peuvent ainsi passer librement dans le cytoplasme.

La ventouse apparaît de très bonne heure; nous avons rencontré des parasites de 6 à $7 \mu$ de diamètre chez lesquels on apercevait à l'un des pôles une cavité dont le fond soulevé en cul de bouteille (fig. 192) rappelait absolument la forme de la cavité de la ventouse adulte. Il s'agit là sans nul doute du début de la formation de cet appareil, le plafond de la ventouse se constituerait ensuite par un repli de l'épicyte se prolongeant au-dessus de cette cavité primitive, simple d'abord, puis lobée (fig. 72).

Les Grégarines de 8 à $10 \mu$ de diamètre présentent toutes une ventouse bien formée (fig. 193).

Le noyau conserve sa forme sphérique primitive pendant toute la durée de la vie du parasite. Nous avons vu qu'il s'accroît d'abord comme chez $M$. agilis dans les mêmes proportions que le corps du parasite, mais la rapidité de sa croissance dimi- 
nue bien vite et l'on peut remarquer en consultant le tableau, page 175 que le diamètre de ce noyau égal au début à la moitié de diamètre du corps, n'atteint bientôt plus que le tiers, puis le dixième de ce même diamètre; le diamètre du karyosome est au début la moitié de celui du noyau, finalement il n'en est plus que le tiers.

Le karyosome, toujours excentrique dans les parasites très jeunes peut conserver cette situation; mais le plus souvent, il émigre vers le centre du noyau. Il est généralement unique; dans quelques cas très rares, il y a deux karyosomes d'égale dimension (fig. 50) et qui proviennent sans doute de la fragmentation d'un karyosome unique. La figure 70 montre un jeune parasite de $9 \mu$. sur $4 \mu .5$ dont le noyau renferme un karyosome formé de deux parties accolées qui se sépareront sans doute ensuite pour donner deux karyosomes

Le karyosome est sphérique comme le noyau; parfois, sa surface est irrégulière, bosselée par une série de grains plus ou moins saillants qui sont bourgeonnés par ce karyosome. C'est une masse vacuolaire qui se teint commela chromatine par la plupart des réactifs qui colorent cette substance. Sa colorabilité varie du reste à chaque instant durant l'évolution du parasite ainsi que celle de l'ensemble du noyau.

Les figures 56 à 65 montrent les divers aspects qu'il peut revêtir ; elles représentent des parasites colorés par l'Hématoxyline à l'alun de fer. Cette méthode a l'inconvénient de ne pas mettre en évidence la structure vacuolaire du karyosome qui paraît ainsi massif; nous avons dessiné à part (fig. 53, 54) des karyosomes colorés par l'Hémalun et qui présentent nettement cette structure, l'un est complètement coloré, l'autre en partie incolore; tous les deux montrent, accolés à leur surface un petit bourgeon qui est en train de se détacher. Chez certaines Grégarines très volumineuses (Grégarines à toison), les vacuoles du karyosome montrent une disposition très régulière (fig. 41) : il existe une sorte de zone médullaire très colorée et une zone périphérique plus claire ; une des vacuo- 
les de cette dernière zone, plus développée que les autres, reniferme en son centre un grain très coloré. Ceci rappelle la figure 4, planche XIII de KuschakewtTSCH; pour cet observateur cette vacuole viendrait crever à la surface pour émettre au dehors le grain qu'elle contient, nous n'avons pas assisté à ce processus.

On voit à l'examen de ces figures que le karyosome peut:

$1^{0}$ Avoir une forte coloration uniforme dans toute sa masse.

$2^{\circ}$ Présenter une région presque incolore et une région fortement colorée, celle-ci étant ou centrale ou excentrique.

$3^{\circ}$ Etre totalement incolore.

A chacun de ces trois aspects correspondent diverses conformations du noyau que nous allons décrire successivement:

I. Le karyosome est fortement coloré. Ce karyosome peut avoir sa surface tout à fait lisse ou présenter un ou plusieurs grains chromatiques accolés; d'autres grains de même volume se trouvent parfois à une faible distance, mais non accolés.

Le noyau peut revêtir dans ce cas, trois aspects :

$1^{\circ}$ Dans les stades jeunes (fig. $42,43,44,73$, etc.), le karyosome est entouré d'une zone claire autour de laquelle sont rangés en cercle de gros grains chromatiques parfois reliés au karyosome par de très fins filaments difficilement perceptibles; le reste du noyau est occupé par des grains plus petits disposés sur un réseau de linine toujours bien visible sur les coupes. La membrane nucléaire est bien colorée. C'est un aspect que nous avons décrit précédemment déjà.

Dans les stades âgés :

$2^{\circ}$ La zone claire est moins nette, il n'y a plus de grains chromatiques régulièrement disposés en cercle ; toute la chromatine est en grains fins dispersés sur le réseau, la membrane nucléaire est bien visible. Nous avons également décrit ce stade (fig. 56, $57,139,140,142)$.

$3^{\circ}$ Le karyosome seul est coloré, le reste du noyau est rempli de granulations incolores; la membrane nucléaire existe ou bien encore elle n'est pas visible (fig. 62). 
II. Le karyosome n'a qu'une région colorée, le reste est incolore.

$1^{0}$ On voit un ou plusieurs gros grains chromatiques tangents au karyosome, d'autres à une faible distance; le reste de la chromatine est sur le réseau. Il y a une membrane nucléaire colorable (fig. 58, 59).

$2^{\circ}$ Le reste du noyau n'est occupé que par un suc nucléaire pâle et il n'y a pas de membrane nucléaire visible (fig. 63, 64).

III. Le karyosome est presque totalement incolore.

1o Il existe un grain chromatique accolé à la surface du karyosome, en outre, de nombreux grains plus petits sont distribués sur le réseau. La membrane nucléaire est bien visible (fig. 60).

$2^{\circ}$ Il y a encore un grain coloré à la surface du karyosome, mais le reste du noyau est presque incolore. Il existe une membrane nucléaire (fig. 61).

$3^{\circ}$ On n'aperçoit plus ni grain accolé au karyosome, ni chromatine dans le noyau; le suc nucléaire est simplement un peu plus coloré que le cytoplasme environnant et il n'y a plus de membrane nucléaire visible (fig. 65).

Nous avons fréquemment observé une structure nucléaire représentée par la figure 52. Du karyosome s'échappent des cordons de chromatine qui envoient dans le noyau des ramifications renflées à leur extrémité ; c'est une forme que nous avons déjà signalée chez $M$. agilis (fig. 16,17 ) et qui rappelle aussi, dans une certaine mesure les figures données par DoGIEL (1907, taf IX, fig. $\mathbf{x}, \mathbf{a}, \mathrm{b}, \mathrm{c})$ pour Schisocystis sipunculi.

Le Mann colore en rouge ou en violet clair (fig. 191, 192), le karyosome des parasites jeunes; à la surface de ce karyosome s'observe une mince couche violette. Les petits grains bourgeonnés par le karyosome sont rouges également. Parfois on voit, accolé à l'un des pôles de ce karyosome un croissant coloré en bleu foncé ou en violet (fig. 187) ou une masse violette presque aussi volumineuse que le karyosome lui-même. Ce sont là 
autant de particularités que nous avons rencontrées chez d'autres espèces, notamment chez $M$. agilis.

La coloration rouge du karyosome ne persiste pas jusqu'à l'accouplement du parasite. Lorsque le cytoplasme devient bleu foncé, puis violet, et qu'il apparaît des gouttelettes rouges dans sa masse, le karyosome se colore en bleu et en violet (fig. 189, 190); mais, alors même qu'il présente cette coloration, on peut voir accolés à sa surface des grains rouges.

Les autres changements d'aspect décrits plus haut, s'ob)servent également avec la méthode de Mann.

Nous n'avons pas observé communément les nombreux aspects de noyaux figurés par DRzewecki (1907) dans les deux planches de son mémoire, sauf dans certains cas de dégénérescence pathologique ou sur les bords de nos frottis en des points où la préparation avait séché avant la fixation.

Les divers faits que nous venons de rencontrer chez St. coronata, nous les avions déjà signalés et étudiés chez $M$. agilis, après Drzewecki (1903). Pendant tout le cours de la vie végétative du parasite, le noyau est en continuelle voie de transformation; chez les stades jeunes, il émet dans le cytoplasme des grains chromidiaux qui jouent probablement un rôle important dans la synthèse des grains de réserve, et, chez l'adulte, prêt à l'enkystement, il envoie à nouveau dans le cytoplasme des grains chromatiques qui serviront de ferments póur la transformation et la digestion de ces grains de paramylon. Chez les jeunes, l'émission des grains chromidiaux a lieu sans amener de modification bien sensible à la structure du noyau ; il en est généralement de même aussi chez l'adulte, cependant dans certains cas, cette émission produit dans le noyau une véritable crise, il y a disparition de la membrane et de toute la chromatine nucléaire ; le noyau ne se différencie plus alors du cytoplasme que par une coloration un peu plus intense du suc nucléaire dans lequel on distingue à peine le karyosome. Le noyau se reconstitue ensuite, et au moment de l'accouplement, il est redevenu normal. 
Dans quel ordre se présentent les divers aspects que nous avons décrits plus haut pour le noyau? Nous ne pouvons indiquer cette succession qu'à titre de simple hypothèse, car elle est déduite de l'examen de nos préparations et non de l'étude in vivo de ces transformations, qui n'est guère possible avec nos moyens d'investigation actuels.

Le noyau normal constitué par un karyosome bien coloré et un réseau de linine sur lequel sont dispersés les grains de chromatine, envoie de la chromatine dans le cytoplasme. Le karyosome bourgeonne des grains qui tombent dans le noyau. Peu à peu noyau et karyosome perdent ainsi leur colorabilité. La membrane nucléaire disparaît pour faciliter des échanges rapides entre le noyau et le cytoplasme. Puis le karyosome se reconstitue et reprend sa colorabilité, la membrane nucléaire reparaît, le karyosome bourgeonne à nouveau des grains chromatiques et le noyau se reforme.

Dans une certaine mesure nos observations confirment celles de Drzewecki sauf en ce qui concerne la disparition totale du noyau qui nous paraît être d'ordre pathologique; mais nous interprétons tout différemment les faits observés.

DRZEWECKI considère les grains colorables qui apparaissent dans le plasma des jeunes parasites comme un substratum contenant de la chromatine non encore tout à fait morphologiquement différenciée. Ces grains " chromatogènes ) se rassemblent en grains plus volumineux les " Nucléolides » qui servent directement à la reconstitution du noyau lorsque celui-ci s'émiette dans le cytoplasme et disparaît totalement. Quant aux chromidies vraies elles n'apparaîtraient que plus tard et DrzewECKI est muet sur leur rôle.

Nous n'avons pas constaté de disparition du noyau dans les jeunes stades, nous n'avons pas vu non plus les grains chromatoïdes disséminés dans le cytoplasme se rassembler en un anneau compact autour du noyau et s'incorporer à sa masse. Pour nous les granulations chromidiales loin de se former dans le cytoplasme seraient au contraire d'origine nucléaire 
et quelques observations viennent à l'appui de notre hypothèse. Ainsi nous avons vu que l'on trouve chez de jeunes parasites, indépendamment du centrosome, un grain chromatique placé sur la membrane et relié encore au karyosome par un fin filament; ce grain tombe ensuite dans le cytoplasme, gardant encore parfois ses connexions avec le karyosome. D'autres parasites montrent un certain nombre de masses chromatoïdes rassemblées à proximité du noyau (fig. 144), alors que le reste du cytoplasme en renferme encore très peu; nous avons même vu une de ces masses paraissant se détacher du noyau (fig. 71). Souvent ces chromidies proches $d u$ noyau sont contenues dans une région cytoplasmique différenciée, en forme de croissant et qui se colore fortement par l'Orange G (fig. 144).

Drzewrekr ne croit pas à l'origine nucléaire de ces masses parce que la chromatine ne diminue pas dans le noyau à ce moment, mais qu'elle augmente au contraire, et parce que le nucléole ne change pas, non plus que le volume du sporozoïte. Mais ces faits s'expliquent facilement si l'on pense qu'il $\mathrm{y}$ a alors nutrition très énergique $d u$ parasite qui peut ainsi remplacer au fur et à mesure la chromatine qui se trouve émise dans le cytoplasme.

Nous avons vu qu'il existe entre la ventouse et le noyau un amas de fines granulations mises en évidence par le Mann et indiquant un courant nutritif intense à ce niveau. Pour nous ces grains seraient des particules destinées à servir à la nutrition du noyau, toutefois nous ne pensons pas qu'elles pénètrent ainsi directement dans le noyau, nous n'avons pas vu celui-ci s'émettre de prolongements amoeboïdes pour saisir ces particules et les incorporer directement à sa masse, nous ne sommes pas en mesure d'indiquer par quel moyen se fait cette incorporation. Nous avons trouvé déjà des granulations semblables dans la trompe de $R$. pilosa. KuschakeWITSCH (1907) en avait signalé auparavant chez les. Grégarines de l'intestin de Tenebrio molitor, mais ces grains allant du noyau 
au septum et pénétrant dans le protomérite seraient pour lui d'origine nucléaire.

Les masses chromidiales n'occupent pas comme ces granulations spéciales une situation fixe, elles peuvent sortir du noyau en un point quelconque de sa surface. Leur présence est sans nul doute aussi en rapport avec le processus de nutrition du parasite; lorsque la nutrition est active, les chromidies sont plus nombreuses et plus volumineuses; c'est ce que l'on observe chez les "Grégarines à toison 》, contenues dans des blastophores très volumineux et entourées par une grande quantité de cellules sexuelles qui leur fournissent un riche matériel nutritif. Les matières nutritives absorbées passeraient dans le noyau où aurait lieu leur transformation, puis le noyau enverrait dans le cytoplasme les éléments destinés à l'élaboration des réserves.

Outre les échanges réciproques entre le cytoplasme et le noyau, il en existe encore entre le noyau et le karyosome, et celui-ci serait en dernière analyse le centre d'élaboration de la chromatine qu'il émettrait par bourgeonnement dans le noyau. Ces échanges ont été signalés déjà chez d'autres Sporozoaires; SiEDLECKI (1905) les a étudiés chez Karyotropha Mesnili, puis Moroff (1906-1907-1908) et LÉGER et DuboscQ (1908) chez les Aggregata.

Le karyosome cède peu à peu sa substance colorable au noyau par une sorte de bourgeonnement; des grains se soulèvent de sa masse, font saillie à la surface puis se détachent. Le karyosome perd ainsi sa colorabilité, quant aux grains émis leur colorabilité change rapidement, d'abord colorés en rouge par le Mann comme la plastine dont ils sont issus, ils deviennent bleu foncé ou violets lorsqu'ils se sont détachés; ces changements s'observent parfois alors même que les grains sont encore accolés à la surface du karyosome. Ainsi les grains émis par le karyosome d'abord colorés comme la pyrénine se colorent ensuite comme la chromatine, c'est ce que nous avons vu déjà à propos de $M$. Le Mêmei et que Moroff (1907) a signalé auparavant chez les Aggregata. 
Pendant que se déroulent tous ces phénomènes, le parasite absorbe non seulement la substance du blastophore dans lequel il est contenu, mais encore une grande partie de celle des cellules sexuelles qui sont fixées sur ce blastophore. Malgré des recherches attentives, nous n'avons pas réussi à voir les spermatozoïdes pénétrer dans le parasite soit par la bouche, soit par un point quelconque de la surface du corps. Nous n'avons pas vu non plus le noyau émettre des pseudopodes pour saisir et incorporer à sa masse les spermatozoïdes ou les grains chromidiaux contenus dans le cytoplasme; la nutrition de St. coronata, quelle que soit la période de son cycle que l'on considère paraît se faire uniquement par voie osmotique comme chez les autres Grégarines; il n'y a pas ingestion de substances solides.

De même que les autres Monocystidées intrablastophoriennes précédemment étudiées, le parasite produit des altérations profondes dans les cellules sexuelles portées par le blastophore envahi, et il en amène toujours le dépérissement. Nous étudierons dans un chapitre spécial ces déformations dont le terme ultime paraît être représenté le plus souvent par de petits blocs de chromatine condensée qui se rassemblent en face de l'ouverture de la ventouse et sont abandonnés au moment où le parasite rompt l'enveloppe du blastophore qui le contient.

Cette enveloppe revêt si étroitement la Grégarine qu'elle semble faire partie intégrante de son corps, de sorte que, sur le vivant, St. coronata paraît très souvent pourvue d'une cuticule couverte de longs poils. La plupart du temps, ces poils sont filiformes, peu épais (fig. 139) ; mais ils peuvent aussi prendre l'apparence de lanières larges et très serrées qui constituent à la Grégarine une toison épaisse d'aspect très particulier (fig. 145) rappelant les formes de $R$. pilosa à poils larges ou de M.hirsuta. Ces poils sont des spermatozoïdes altérés et les divers aspects qu'ils présentent correspondent comme nous le verrons à divers processus d'altération.

Les phénomènes pathologiques que nous arons signalés 
chez d'autres Monocystidées se retrouvent également ici : englobement par les phagocytes de l'hôte, et attaque par des Bactéries endoparasites; nous les décrivons plus loin en détail.

St. coronata une fois sortie de son enveloppe peut continuer à grossir encore avant l'enkystement; elle se nourrit sans doute aux dépens du fluide séminal, mais toujours par osmose. Nous n'avons pas remarqué qu'elle se suspende par son péristome à la paroi des vésicules séminales comme le signale Drzeweckr; elle est toujours libre dans la lumière de ces organes au milieu des follicules spermatiques.

\section{Stomatophora diadema n. sp.}

J'ai décrit chez Helodrilus longus Ude une Grégarine qui paraît presque constamment entourée par les phagocytes de l'hôte, cette particularité remarquable de $N$. vermicularis se retrouve chez une espèce parasite de $P$ h. hawayana et d'un $P h$. sp. indét, et à laquelle nous avons donné le nom de $S t$. diadema.

Duanose. - Corps hémisphérique présentant des stries méridiennes régulières et des sillons méridiens profonds séparant des lobes parfois très accentués. Atteint jusqu'à 105 \%. de diamètre. Le sommet du dôme est occupé par une cavité cratériforme qui constitue une sorte de ventouse. Mouvements peu marqués : cyclose, dilatation et rétraction des lobes. Noyau excentrique placé entre le pourtour de la ventouse et la surface du corps, présentant un gros karyosome et quelques grains chromatiques sur un réseau.

Kystes généralement sphériques de 45 \%. à 50 ". de diamètre et ayant une paroi assez épaisse à double contour très net.

Sporocystes allongés, naviculaires de 12 à $15 \mu$ de long sur 5 à $6 \mu$ d'épaisseur, renfermant 8 sporozoïtes.

Hôtes : Ph. hawayana et Ph. sp.

Stomatophora diadema est très rare, nous ne l'avons observée que deux fois : chez un Pheretima hawayana Rosa et chez un ARCH, DE ZOOL。 EXP. ETT GẼN, - 5 SERRIE, - T. IIr. - (II). 
autre Pheretima que nousn'avons pu identifier. Les deux hôtes provenaient des serres du Jardin des Plantes de Grenoble, leurs vésicules séminales renfermaient un très petit nombre de parasites, aussi ne nous a-t-il pas été possible d'étudier ceuxci en détail.

St. diadema a la forme d'un dôme ou d'une hémisphère dont le sommet est occupé par une sorte de ventouse. La surface du parasite est creusée de sillons méridiens très profonds qui le découpent en lobes irréguliers dont quelques-uns sont parfois très développés (fig. 66, 67, 68).

Les dimensions sont très variables, le diamètre moyen est de 70 \%. mais certains parasites ont jusqu'à $105 \mu$. La hauteur est en général égale à la moitié du diamètre, sauf chez les formes à très grand diamètre qui sont fortement surbaissées.

Les mouvements sont lents et peu marqués : les granulations cytoplasmiques se promènent en cercle, en outre il y a quelquefois dilatation et rétraction des lobes.

La ventouse occupe le sommet du dôme; c'est une grande cavité conique ou cratériforme parfois assez profonde. Le diamètre de l'ouverture est égal au tiers, souvent même à la moitié du diamètre de la Grégarine. Le rebord circulaire de l'ouverture est coloré par le Fer avec une grande intensité. Les parois sont ornées de lignes génératrices également colorées par le Fer. Quelques-unes de ces lignes sont situées sur le prolongement des sillons creusés à la surface du parasite, elles sont très accentuées ; d'autres moins marquées font suite aux stries épicytaires.

De même que chez St. coronata, le fond de la cavité se soulève en une petite éminence conique qui se colore assez fortement par le Fer ou par les colorants cytoplasmiques, le Vert lumière par exemple. Il n'y a jamais ici de plafond, par conséquent la colerette pétaloïde si curieuse de St. coronata n'existe pas.

Les formations ectoplasmiques sont très réduites. L'épicyte est orné de très fines stries méridiennes parallèles aux sillons qui séparent les lobes. Le sarcocyte est excessivement mince, je n’ai pas observé le myocyte. 
Le cytoplasme endoplasmique finement granuleux est creusé de larges alvéoles où se logent les grains de paramylon.

Le noyau a toujours une situation excentrique, il est placé entre la ventouse et le bord. Il renferme un gros karyosome central entouré par une zone claire et quelques fines granulations chromatiques.

Les kystes sont le plus souvent sphériques, parfois cependant allongés en ellipsoïdes, ils mesurent en moyenne 45 à $50 \%$ de diamètre. Leur paroi épaisse montre toujours nettement un double contour. Ils renferment des sporocystes naviculaires allongés ayant la forme et la structure typique de ceux des Monocystis des Lombriciens et dont les dimensions varient entre 12 et 15 u. pour le grand axe et 5 à $6 \mu$ pour le petit axe (fig. LXxxIII).

Je ne connais pas les stades jeunes de cette espèce.

Les adultes sont rarement libres dans les vésicules séminales, je les ai presque constamment observés recouverts par une épaisse couche de phagocytes de l'hôte.

\section{Pleurocystis Cuenoti n. sp.}

Cuḱnot (1901) a découvert et figuré (pl. XVIII, fig. 5) des pąrasites qu'il nomme $M$. magna et qu'il a trouvés réunis par couples attachés aux saillies cupuliformes des pavillons séminaux ciliés de $L$. herculeus Sav., et de Allobophora terrestris Sav.

Nous avons rencontré chez Helodrilus longus Ude et Helodrilus caliginosus Sav. de la Haute-Saône et du Dauphiné une Grégarine absolument semblable à celle qui a été dessinée par Cú́not ; elle vit toujours assemblée par couples situés comme Nematocystis magna dans les replis des pavillons séminaux. Elle se rapproche de cette espèce par cet habitat spécial, par sa forme cylindroïde allongée, ses grandes dimensions, ses mouvements, sa couleur d'un blanc éclatant; mais elle s'en éloigne manifestement par son mode de fixation, la structure de sa cuticule, la pluralité de ses karyosomes et par sa disposition par paires rappelant l'accouplement précoce de Zygocystis 
cometa, des Gamocystis, etc. En outre nous ne l'avons jamais rencontré chez Lumbricus terrestris. Pour ces raisons nous la distinguerons de $N$. magna et nous l'appelons Pleurocystis Cuenoti, la dédiant au savant qui l'a observée en premier lieu.

Diagnose. - Monocystidée de grande taille (jusqu'à $2 \mathrm{~mm}$. de long sur $300 \mu$ d'épaisseur) vivant par couples à accolement longitudinal. Corps vermiforme, d'un blanc éclatant, très déformable et souvent recouvert par une couche de spermatozoüdes de l'hôte piqués normalement à sa surface et lui formant un revêtement pileux. Extrémité antérieure renflée et enserrée dans une sorte de coupe constituée par un amas de cellules épithéliales des pavillons séminaux fortement hypertrophiées et faisant saillie sous forme de massues à la surface de l'épithélium. Epicyte orné de stries larges, assez éloignées les unes des autres, disposées en hélice dans la région postérieure du corps. Fibrilles du myocyte très fines et peu visibles. Noyau à plusieurs karyosomes ou à karyosome unique moniliforme.

Hôtes : Helodrilus longus Ude et Helodrilus caliginosus Sav.

Pleurocystis Cuenoti vit fixée à la paroi des pavillons séminaux comme $N$. magna à laquelle elle ressemble; on ne la rencontre jamais dans les poches séminales. Elle paraît propre à deux espèces d'Helodrilus : $H$. longus Ude et $H$. caliginosus Sav. où elle est rare. Je ne l'ai jamais observée dans d'autres hôtes.

Sa forme rappelle celle des Grégarines allongées précédemment décrites; elle est cylindrique ou cylindro-conique. De même que $R$. pilosa, elle est en général renflée au pôle antérieur et s'amincit en allant vers l'autre extrémité, elle s'éloigne ainsi de $N$. magna qui s'atténue à ses deux extrémités. Par contre, elle peut comme cette dernière espèce présenter une série de constrictions qui lui donnent un aspect moniliforme plus ou moins régulier.

$P$. Cuenoti ne se trouve jamais isolément, on rencontre toujours deux parasites fixés côte à côte et accolés longitudina- 
lement sur la plus grande partie de leur longueur, seules les extrémités postérieures sont divergentes (fig. Lxxxiv).

Les dimensions du parasite peuvent devenir considérables; toutefois, elles semblent toujours rester inférieures à celles de $N$. magna. Les plus grandes Grégarines que j'aie vues ne dépassaient pas $2 \mathrm{~mm}$. de longueur; le diamètre par contre est plus grand que celui de $N$. magna, il atteint parfois jusqu'à $300 \mu$.

Les mouvements sont absolument comparables à ceux de $N$. magna.

La partie antérieure de $P$. Cuenoti est enserrée d'une façon assez lâche dans une sorte de cavité cupuliforme analogue à celle qui coiffe la tête de $N$. magna, mais formée ici, non plus comme chez Lumbricus terrestris par une cellule unique, mais par un nombre considérable de cellules en massue disposées côte à côte en bouquet, et dont chacune a à peu près le même aspect que la cellule en bocal qui sert à la fixation de $N$. magna. Ces cellules souvent étroitement accolées ne sont jamais fusionnées; elles présentent un noyau volumineux et sont couvertes de longs cils épais à leur base tout à fait comme la cellule cupuliforme jeune. Ces cils manquent sur la surface de contact entre la cellule et le parasite; là, des sillons creusés dans la cellule reçoivent les côtes longitudinales qui ornent

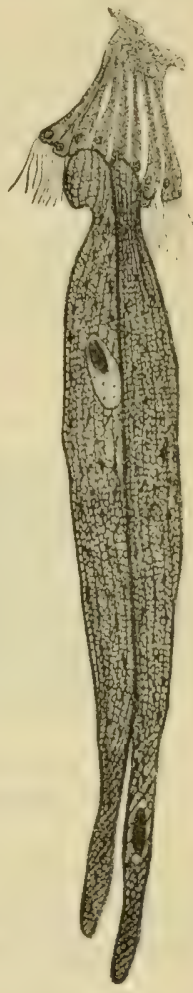

FIG. LXXXIV. P. Cuenoti accolées à des celIules hypertrophiées du pavillon séminal $x$ 280 . la surface de la Grégarine dont la fixation est ainsi assurée de la même façon que pour N. magna; mais ici il n'existe jamais d'épimérite différencié enfonçant ses racines piliformes dans le corps des cellules support.

Ces côtes s'atténuent rapidement vers l'arrière et se continuent par les stries de l'épicyte. Celles-ci, assez larges, éloignées 
les unes des autres, parallèles, sont disposées selon des lignes méridiennes sur la région médiane du corps ; sur la région postérieure à partir du point de divergence des deux parasites accolés, elles s'enroulent en hélice, ce qui semblerait indiquer une torsion de cette partie du corps des Grégarines.

La couche de sarcocyte est peu épaisse et semble d'égale épaisseur sur tout le corps.

Les fibrilles du myocyte, très fines, ne sont visibles que dans les régions où le corps s'est fortement contracté ; il en est presque toujours ainsi dans les queues divergentes des parasites. En ces points, l'ectoplasme montre des plissements en accordéon

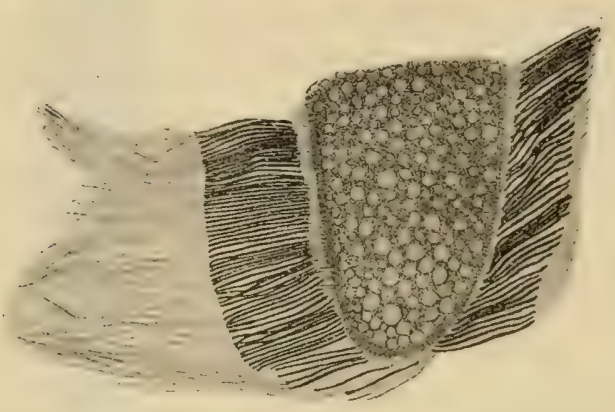

FIG. LXXXY. Extrémité postérieure de $P$. Cuoneti couverte de spermatozoīdes de l'hôte $\times 1000$. tout à fait caractéristiques et que nous avons trouvés déjà chez d'autres Monocystidées. Ces plissements se présentent en coupe optiquecomme depetits bâtonnets cylindriques courts et très serrés (fig. Lxxxv).

Le corps de $P$. Cuenoti est très souvent recouvert de spermatozoïdes de l'hôte qui lui forment un revêtement pileux complet. Cú́not (1901) a óbservé ce fait et figuré deux parasites avec une semblable toison (pl. XVIII, fig. 5). Ces spermatozoïdes sont rangés très régulièrement les uns à côté des autres, étroitement tassés, la tête tournée vers la cuticule du parasite, la queue vers l'extérieur; ils présentent ainsi la même disposition que dans les pavillons ciliés. Les queues divergentes des parasites qui sont souvent contractées ainsi que nous l'avons vu, présentent plus communément ce revêtement que le reste du corps; les "Spitzenstück " des spermatozoïdes sont alors piqués entre les plis de l'ectoplasme de la même façon qu'ils s'implantent entre les cils de l'épithélium dans les pavillons 
(fig. Lxxxv). La présence des plis cuticulaires n'est du reste pas indispensable pour la fixation des spermatozoïdes; car ceux-ci s'observent fréquemment avec une disposition tout à fait semblable en deźrégions où le corps n'est pas contracté. Ils sont sans doute retenus par une mince couche mucilagineuse parfois lisse, parfois découpée en courts filaments qui recouvre la cuticule des Grégarines. Je n'ai pu déterminer l'origine de ce mucus et ne sais si c'est une sécrétion propre au parasite ou une simple accumulation à sa surface de fluide séminal de l'hôte.

L'endoplasme granuleux est creusé d'alvéoles de taille variable dans lesquelles sont logés les grains de paramylon ellipsoïdaux et de volume très irrégulier. Les cordons du réseau cytoplasmique qui entourent les alvéoles ne présentent pas une épaisseur uniforme, il y a en certains points des accumulations d'un cytoplasme dense et à granulations plus fines.

Le noyau n'occupe pas de situation fixe, il paraît se promener d'une extrémité du corps à l'autre, ballotté avec les granulations endoplasmiques et les grains de réserve par les mouvements du parasite. Cú́not représente les noyaux des deux syzygites en face l'un de l'autre, ce n'est pas là un fait constant; ces noyaux peuvent aussi se trouver éloignés l'un de l'autre, placés parfois même à deux extrémités opposées.

Le noyau est un ellipsoïde volumineux renfermant en général plusieurs karyosomes sphériques ou irréguliers. La membrane nucléaire n'est pas très colorable.

Lorsqu'il n'y a comme chez $N$. magna qu'un seul karyosome très volumineux, au lieu d'être sphérique comme chez cette espèce, il est irrégulier, moniliforme, présentant comme un début de fragmentation; on y observe diverses masses compactes, alvéolaires avec une croûte plus colorée, séparées par des bandes plus étroites, remplies de fines granulations chromatiques.

Le Mann décèle dans ces karyosomes une structure très curieuse. Parfois comme chez les autres Monocystidées des 
Oligochètes, le karyosome est une masse vacuolaire complètement colórée en rouge, sauf une mince croûte violette; accolés à cette croûte, on aperçoit de gros grains rouges; d'autres grains de même volume et de même coloration se voient dans les mailles du réseau de linine qui remplit le reste du noyau et sur lequel sont dispersées de fines granulations violettes (fig. 178). D'autres fois, le centre du karyosome est violet, une zone rouge l'entoure et sur celle-ci s'étend la croûte violette (fig. 179). De petits grains bleus sont disposés sur le réseau de linine, mais il n'y a pas de grains rouges dans les mailles de ce réseau. Enfin quelquefois la plus grande partie du karyosome est colorée en violet, tout autour s'observe une bordure rouge d'où irradient des branches plus ou moins ramifiées qui se rendent jusqu'à la membrane nucléaire. Le réseau de linine présente la structure ordinaire (fig. 177).

On voit que le karyosome de $P$. Cuenoti rappelle, par sa complexité tout au moins, celui que LÉGer et DuboscQ (1908) ont décrit chez les Aggregata, il eût été intéressant de suivre son évolution durant le développement du parasite, malheureusement $P$. Cuenoti est rare et je n'ai pu observer aucun stade de son développement n'ayant eu sous les yeux que des formes adultes.

J'attribue à cette espèce des kystes de $1 \mathrm{~mm}$. 5 à $2 \mathrm{~mm}$. de diamètre trouvés dans les segments génitaux de Helodrilus longus et de $H$. caliginosus et renfermant des sporocystes de $28 \mu .5$ sur $12 \mu$.

\section{Zygocystis cometa Stein.}

Cette Grégarine a été découverte, décrite et figurée par STEIN (1848). Depuis, un grand nombre d'observateurs l'ont revue et figurée à nouveau. Pour Schmid (1854), cette espèce n'existerait sûrement pas chez $L$. agricola où STEIN prétend l'avoir rencontrée, RuschHAupt (1885) au contraire l'aurait trouvée, une seule fois, chez cet hôte. Je n'ai pas été plus heureux que Schmid et n'ai jamais observé cette espèce dans les 
Lumbricus, par contre elle est assez abondante chez certains Helodrilus, $H$. longus Ude, $H$. caliginosus Sav. et surtout $H$. caliginosus var. trapezoides Ant. Dugés.

Diagnose. - Monocystidée ovoïde ou piriforme, vivant constamment en association : deux individus étant accolés par leur pôle antérieur arrondi. Le pôle postérieur porte une touffe de lanières assez développées qui sont le prolongement des côtes ectoplasmiques de la surface du parasite. Ces côtes sont méridiennes, et séparées par de larges et profonds sillons; elles sont constituées surtout par du sarcocyte. Endoplasme dense à la périphérie mais renfermant vers le centre de grandes vacuoles qui disparaissent à l'approche de l'enkystement. Grains de paramylon sphériques de taille excessivement variable.

Kystes sphériques ou ellipsö̈daux. Sporocystes naviculaires ayant la forme et la structure des sporocystes des Monocystis.

Hôtes : $H$. longus Ude, $H$. caliginosus Sav. et $H$. caliginosus var. trapezoides Ant. Duges.

Habitat et StÈGE. - Ce parasite est très commun dans les vésicules séminales des Helodrilus, : $H$. longus, $H$. caliginosus et sa variété trapezoides. Je ne l'ai jamais observé chez les Lumbricus.

Dimensions, forme et mouvements. - Il vit presque toujours en associations de deux individus accolés par leur pôle antérieur arrondi et paraissant totalement immobiles (fig. Lxxxvi). Cependant on observe de temps en temps quelques individus jeunes isolés; on rencontre aussi quelquefois des associations de trois individus, telles que celle représentée par Stein (1848, taf IX, fig. 7).

Si l'on fait abstraction de la touffe de digitations qui le termine postérieurement un individu considéré isolément est piriforme quand il est jeune, puis il devient ovoïde et ellipsoïdal au moment de l'enkystement; la dimension de l'axe antéropostérieur est d'abord la plus développée, puis peu à peu c'est l'axe perpendiculaire qui l'emporte; et, dans des couples 
s'enkystant, nous relevons les dimensions suivantes : largeur (axe d'accouplement) $135 \mu$; longueurs : $120 \mu+115 \mu$; ou encore largeur, $115 \mu$; longueurs : $105 \mu+100 \mu$. Les conjoints comme on le voit ne sont pas rigoureusement de même taille, leur longueur est rarement la même, leur largeur même peut varier de façon notable (voir fig. 6, taf $\mathrm{Ix}$ de Stein).

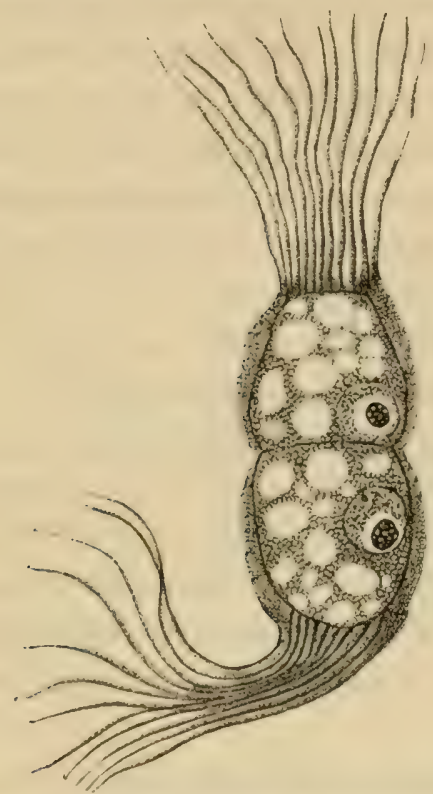

L.XXVII.

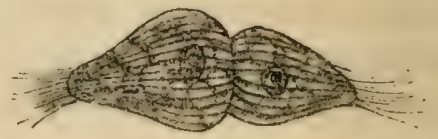

$\angle X X X V T$

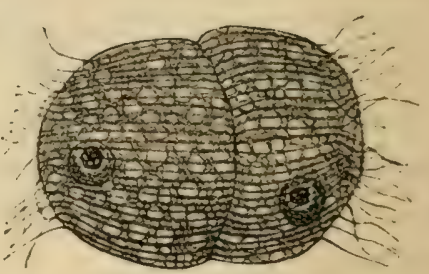

LXXXVIII

FIG. LXXXVI. Z, cometa jeunes $\times$ 250.-FiG. LXXXVII. Z, cometa. Coupe d'un couple jeune $\times 650 .-$ FIG. LXXXVIII. Z. cometa; couple à la veille de l'enkystement $\times 250$.

L'ectoplasme est épais, creusé de sillons profonds et larges qui séparent des crêtes méridiennes de sarcocyte bien développées et dont le sommet se colore fortement par le Fer. Ce sont ces crêtes qui se continuent en arrière du corps pour constituer le toupet chevelu postérieur caractéristique de l'espèce. Ces lanières postérieures sont parfois très allongées ; dans les formes jeunes, notamment, leur longueur peut dépasser notablement celle du corps lui-même (Fig. Lxxxvir).

L'endoplasme est creusé de grandes vacuoles, en général 
plus nombreuses vers le centre du corps. Ailleurs, il est alvéolaire et renferme des grains de paramylon sphériques, de taille très variable, comme on peut le voir sur la figure Lxxxvir. Ces vacuoles n'ont pas été signalées par les auteurs; Conorhynchus echiuri Greef (1880), est la seule Grégarine chez laquelle on en ait observé jusqu'ici.

Le noyau sphérique et logé vers le centre du corps, renferme un.gros karyosome vacuolaire.

A l'approche de l'enkystement, les vacuoles du cytoplasme disparaissent; l'épaisseur de l'ectoplasme diminue, les sillons, qui présentaient dans les individus jeunes à peu près la même largeur que les crêtes, s'élargissent considérablement, les crêtes demeurant étroites; et leur sommet devenant encore plus colorable; les lanières terminales deviennent filiformes; au lieu d'être très rapprochées et de former une sorte de toupet, elles s'écartent les unes des autres et semblent s'insérer sur le corps suivant une ligne circulaire limitant un dôme (fig. Lxxxvin), puis elles s'atrophient et disparaissent. On voit que ce processus s'éloigne notablement de celui qui est figuré par Stein (1848, pl. IX, fig. 9 et 10); je n'ai pas retrouvé en effet le mode de disparition de la queue qu'il représente.

Le volume du noyau diminue par rapport à celui du karyosome. Celui-ci émet dans le suc nucléaire de nombreuses petites boules chromatiques. Puis la paroi kystique est sécrétée. Je n'ai pas suivi les phénomènes de la sporulation qui semblent conformes dans leurs grandes lignes à ceux que BrasiL (1905 b) a indiqué pour les Monocystis de $L$. herculeus.

\section{Zygocystis pilosa n. sp.}

Outre $Z$. cometa Stein, les vésicules séminales de Helodrilus longus Ude, renferment encore une autre Grégarine du même genre, mais qui diffère de $Z$. cometa en ce que le corps tout entier est couvert d'expansions en lanières et non plus seulement l'extrémité postérieure. Nous donnons pour ce fait au nouveau parasite le nom de Z. pilosa $\mathbf{n}$. sp. 
Diagnose. - Monocystidée toujours en association de deux individus. Couples ovoüdes, presque sphériques. Pas de stries épicytaires visibles; corps couvert de poils larges et allongés. Cytoplasme creusé d'alvéoles très inégales. Grains de paramylon sphériques. Noyau sphérique peu volumineux, avec un gros karyosome. Sporocystes typiques des Monocystis.

Hôte: Helodrilus longus Ude.

Z. pilosa n'est pas aussi commune que Z. cometa; je n'ai rencontré ce parasite que dans quelques exemplaires d'Helodri-

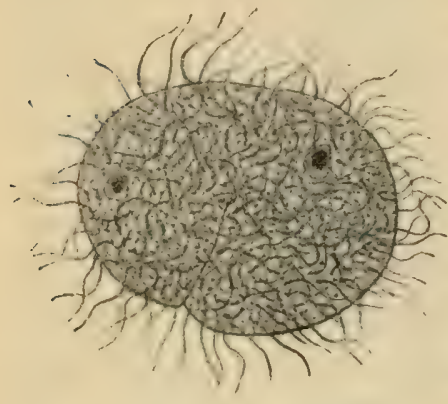

FIG. LXXXIX. Z. pilosa $\times 250$.

lus longus récoltés en automne dans la Haute-Saône, je ne l'ai pas retrouvé en Dauphiné.

Je n'ai jamais observé comme chez les autres Zygocystis d'individus isolés, tous vivent en association, formant des couples ovoïdes, presque sphériques, pouvant atteindre jusqu'à $200 \mu$ de diamètre. Souvent l'un des syzygites est un peu plus grand que l'autre.

Les deux conjoints sont toujours accolés par une large base et se dépriment mutuellement (fig. Lxxxix), au contraire de ce qui a lieu chez $Z$. cometa où ce fait ne se produit qu'au moment de l'enkystement, les conjoints étant, au début, simplement tangents par leur pôle antérieur.

Les mouvements sont très lents, ou nuls.

L'ectoplasme est très mince. On n'observe pas, à la surface du corps d'épaisses crêtes de sarcocyte comme chez Z cometa; il n'y a pas d'autre ornementation visible que des poils larges, allongés, sortes de lanières dont l'axe se colore fortement par le Fer, les bords paraissant hyalins se teintent au contraire par le Vert lumière. Avec la méthode de Mann, les bords de ces poils sont teintés en bleu, l'un plus fortement que l'autre, tandis qu'au contraire l'axe, rougeâtre paraît 
occupé par un cytoplasme granuleux, vacuolaire (fig. 186).

Le cytoplasme renferme des alvéoles de taille très inégale qui contiennent les grains de paramylon sphériques, les uns volumineux, jusqu'à $9 \mu$ de diamètre, les autres, très petits, n'atteignant pas $1 \mu$.

Le réticulum spongioplasmique est formé de petits grains colorés en gris par le Fer. En certains points, on constate une accumulation de grains plus gros, sphériques, parfois colorés fortement par le Fer, parfois au contraire par le Vert lumière. Le Mann les colore en bleu (fig. 186).

Le noyau sphérique peu volumineux est entouré d'une membrane chromatique; il renferme un karyosome très gros, sphérique, vacuolaire, coloré en rouge par le Mann, avec une croûte superficielle violette (fig. 186).

Il existe en outre des grains de chromatine assez gros dispersés sur le réseau de linine.

Je ne connais rien du développement de l'espèce.

Zygocystis Legeri n. sp.

J'ai rencontré dans les vésicules séminales de quelques Helodrilus chloroticus Sav. récoltés à Montessaux, une nouvelle espèce de Zygocystis que je nomme Z. Legeri, la dédiant à mon Maître, M. le professeur Léger.

Diagnose. - Monocystidée vivant presque constamment en association, deux individus s'accolant par leur pôle antérieur. L'un deux présente une sorte de prolongement conique qui s'enfonce dans une dépression correspondante du conjoint. Chaque associé est piriforme et montre un pôle postérieur terminé en pointe allongée, souvent très mince. La longueur d'un individu peut atteindre jusqu'à $150 \mu$, l'épaisseur étant de 40 à $45 \mu$. Cuticule striée en long ; fibrilles du myocyte très fines et très serrées, les unes transversales, les autres longitudinales, celles-ci plus serrées. Sarcocyte peu développé. Endoplasme alvéolaire. Grains de paramylon sphériques de dimensions diverses. Noyau à un seul karyosome volumineux. 
Kystes sphériques, renfermant des sporocystes naviculaires de forme et de structure semblables à celles des sporocystes de Z. cometa et mesurant $15 \mu$ sur $4 \mu 5$.

\section{HôтE : Helodrilus chloroticus Sav.}

Haвitat et SIÈGE. - Zygocystis Legeri habite les vésicules séminales de Helodrilus chloroticus Sav. Les hôtes infestés sont rares; ainsi je n'ai jamais trouvé de parasite dans les Helodrilus chloroticus recueillis dans le Dauphiné ; par contre, ceux que j'ai recueillis dans la Haute-Saône renfermaient de nombreuses Grégarines à divers stades de. leur développement. Les $H$. chloroticus des serres du Jardin des Plantes de Grenoble ne m'ont jamais montré $Z$. Legeri, mais on y trouve $M$. Cognettii décrite dans un précédent chapitre.

Dimensions, forme, Mouvenents. - $Z$. Legeri, de même que l'espèce génotype $Z$. cometa, vit presque constamment en association. On observe deux individus accolés par leur pôle antérieur. Tous deux sont piriformes et présentent une extrémité postérieure longuement acuminée

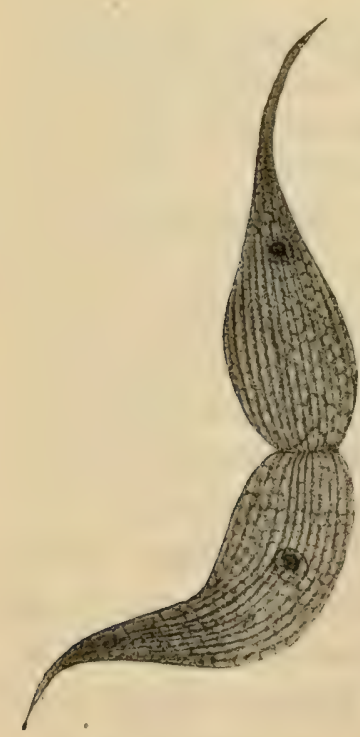

Fla. XC. Z. Legeri $\times 250$. en une pointe aiguë. L'un d'eux a le pôle antérieur arrondi et déprimé en son centre ; l'autre au contraire montre au milieu de ce pôle antérieur une éminence conique qui s'enfonce dans la dépression correspondante du conjoint (fig. Xc). Ces formes spéciales du pôle antérieur se retrouvent dans les quelques rares individus isolés que j'ai pu observer.

Le couple présente une longueur totale qui peut atteindre jusqu'à $300 \mu$, avec une épaisseur de $45 \mu$. Au moment de l'enkystement, la région caudale se reploie sur le reste du corps, avant la sécrétion de la paroi kystique 
de sorte que les kystes montrent une forme sphérique régulière.

Les mouvements du parasite sont assez vifs ; ils sont surtout manifestes dans la région postérieure et rappellent ceux de M. lumbrici; en outre la queue peut se tordre et s'enrouler en divers sens (fig. xcIII).

Nous venons de voir qu'il existe une différence de forme manifeste entre les deux pôles du parasite; cependant, ici, pas plus que chez $Z$. cometa, nous n'avons pu retrouver le mucron épiméritique qui existe si souvent chez les Monocystis.

L'ectoplasme de Z. Legeri est divisé en bandes longitudinales séparées par des sillons assez profonds, qui se colorent fortement par les colorants nucléaires. Il n'y a pas d'autre ornementation épicytaire. Les bandes longitudinales d'ectoplasme sont constituées par le sarcocyte dont l'épaisseur est ainsi loin d'être uniforme sur toute la surface du parasite.

Il existe ici deux systèmes de fibrilles myocytiques, comme nous en avons trouvé déjà chez quelques Monocystis, les unes longitudinales, les autres circulaires et transversales, perpendiculaires aux premières. Toutes ces fibrilles sont fines et serrées, sensiblement parallèles les unes aux autres dans chaque système.

L'endoplasme est creusé d'alvéoles de taille très inégale, où sont logés les grains de paramylon. Ceux-ci sont sensiblement sphériques, les uns petits, les autres assez volumineux. Les mailles de spongioplasme qui séparent les alvéoles sont formées par des grains de deux sortes, les uns gros, sphériques, se colorent en rouge par le Mann; les autres, plus petits, irréguliers se colorent au contraire en bleu ou en violet.

Le noyau, sphérique, contient un karyosome volumineux vacuolaire, qui se teint en rouge par le Mann, et un réticulum lininien sur lequel sont dispersés des grains chromatiques assez nombreux.

Nous avons dit qu'il y a entre les deux associés une différence de forme qui peut être considérée comme une différenciation sexuelle, l'un des individus (fig. xcI) présentant une 
sorte de rostre qui s'enfonce dans le pôle antérieur de l'autre (fig. XcII), Mais on ne retrouve pas ici la différence de structure des cytoplasmes si manifeste chez $M$. Cognettii.

$\mathrm{Au}$ moment de l'enkystement, nous avons vu que la queue

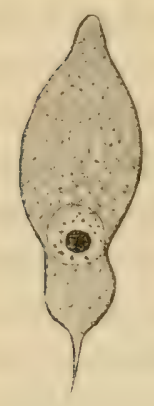

$X C /$

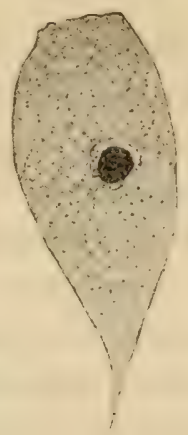

XC:II

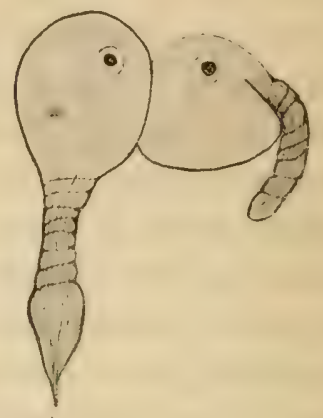

XCIII.

FIG. XCI, Z, Legeri $\times 650$. Un jeune individu $\sigma^{7}$ isolé. - FIG. XCII, Z. Legeri $\times 650$. Un jeune individu ○ isolé, - FıG. XCIII. $Z$. Legeri $\times 250$, couple en voie d'enkystement.

effilée des parasites se ratatine et se rabat sur le corps (fig. XcrII), de sorte que l'ensemble des deux conjoints constitue un ellipsoïde ou une sphère autour de laquelle se forme l'enveloppe kystique. Les sporocystes sont naviculaires, étroits et allongés, ils mesurent $15 \mu$ sur $4 \mu .5$.

J'ai observé quelques syzygites très jeunes, mesurant seulement une quarantaine de $\mu$; ils sont libres dans le fluide séminal. Il semble donc qu'il n'existe pas ici de stade intracellulaire.

\section{CHAPITRE III}

\section{ÉTUDE COMPARÉE}

Nous allons résumer en quelques pages l'étude des nombreuses espèces de Monocystidées hébergées par les Oligochètes. Tout en rappelant leurs divers caractères, nous pourrons en les comparant donner un court aperçu de la morphologie et de la cytologie comparée de ces organismes. 


\section{Morphologie.}

Forme. - Ces parasites présentent une grande variété de formes. Nous avons vu déjà que la distinction d'un certain nombre de genres est basée uniquement sur cette diversité des formes. On peut distinguer deux types extrêmes entre lesquels il existe toute une série d'intermédiaires ; ces formes fondamentales sont la sphère et le cylindre long et de faible diamètre ; $M$. herculea, $M$. Cognettii, $M$. villosa, M. lumbrici olidi, M. Michaelseni, M. turbo, St. coronata, etc., sont des formes sphériques; d'autres parasites, encore peu allongés ont cependant un axe un peu plus développé que l'autre, ce sont des Grégarines ovoïdes ou fusiformes, telles M. Le Mêmei, M. Bretscheri, M. lumbrici, M. agilis. M. Elmassiani est très allongée, tout en restant fusiforme ; quant aux espèces du genre Nematocystis : N. magna, $N$. vermicularis, $N$. lumbricoides, $N$. anguillula, elles ont absolument l'aspect de Nématodes, c'est-à-dire de cylindres très allongés et à extrémités arrondies ou légèrement amincies. Entre ces formes éloignées, nous trouvons des intermédiaires représentés par $R$. pilosa et $R$. porrecta qui sont ou des ovoïdes courts ou de très longs fuseaux semblables à $M$. Elmassiani.

Tous les parasites qui habitent purement la cavité générale, paraissent se rattacher au type sphérique ; quant aux Monocystidées séminales, elles sont tantôt sphériques, tantôt cylindriques. Chaque espèce d'Oligochète paraît héberger des Grégarines des deux formes: chez les Lombriciens, les Grégarines sphériques, moins volumineuses, habitent les vésicules séminales; les Grégarines cylindriques, parfois très développées sont fixées aux replis de la paroi des pavillons ciliés; telles sont $N$. magna et une forme très rare observée chez Octolasium lacteum et que nous nommerons $N$. elongata; on peut cependant rencontrer aussi quelques espèces dans les vésicules séminales ou dans la cavité générale par exemple $N$. lumbricoides et $N$. vermicularis. Chez les Pheretima, les deux formes 
cohabitent dans les vésicules séminales; nous n'avons pas observé de parasites appendus aux pavillons séminaux.

Les espèces de Monocystidées dont nous venons de parler restent solitaires pendant toute la durée de leur vie végétative; chez les Pleurocystis et les Zygocystis au contraire, on constate un accouplement précoce et les individus vivent constamment en association. Les Zygocystis n'atteignent jamais de très grandes dimensions, en outre, ils sont en général peu allongés, ce sont des ovoïdes voisins de la sphère. Quant aux Pleurocystis, ils sont très longs et cylindroïdes. On pourrait en somme considérer les Zygocystis comme des Monocystis en syzygie, les Pleurocystis étant au contraire des Nematocystis associées. Entre les deux, Z. Legeri pourrait être considérée comme une sorte de type intermédiaire, de même que les Rhynchocystis pilosa et $R$. porrecta sont intermédiaires entre les Monocystis et les Nematocystis.

TAILle. - Nous avons noté entre les diverses espèces des différences de taille considérables, on observe done à ce point de vue une variété aussi grande que pour la forme. Alors que $M$. turbo ne dépasse pas $100 \mu$ de diamètre, certaines $N$. magna ou $\mathrm{Pl}$. Cuenoti ont jusqu'à $5 \mathrm{~mm}$. de long sur 40 à $50 \mu$ d'épaisseur. On observe même de notables différences de volume chez une seule et même espèce, différences en rapport avec l'hôte ou avec le mode de développement du parasite. Ainsi, nous avons vu que les $M$. lumbrici hébergées par $L$. rubellus sont beaucoup plus volumineuses que celles que l'on rencontre chez $L$. herculeus et surtout chez $L$. castaneus. En général la taille du parasite n'est pas corrélative de celle de l'hôte : les jeunes Oligochètes donnent asile à des Grégarines aussi volumineuses que les Oligochètes adultes, les $L$. rubellus plus petits que $L$. herculeus renferment des $M$. lumbrici beaucoup plus volumineuses; cependant chez $L$. castaneus qui est de petite taille les $M$. lumbrici et surtout $M$. agilis sont moins grandes que chez $L$. rubellus et $L$. herculeus. Nous savons également que, dans un même hôte, Pheretima hawayana par exemple, 
la plupart des St. coronata n'ont pas plus de 60 à 80 u.; mais que, certaines d'entr'elles (Grégarines à toison) qui ont envahi de très bonne heure le follicule spermatique peuvent grandir considérablement et atteindre jusqu'à $180 \mu$ sur $130 \mu$. Un fait analogue se retrouve chez Helodrilus caliginosus d'El-Kantara où les $M$. Le Mêmei vivant dans les vésicules séminales mesurent seulement $200 \mu$ sur $120 \mu$ en moyenne, tandis que les mêmes parasites dépassent $650 \mu$ sur $300 \mu$ lorsqu'on les rencontre dans le coelome.

Mouvements. - La plupart des Monocystidées que nous avons étudiées sont mobiles et nous retrouvons dans la modalité du mouvement la même variabilité que dans la forme et le volume. M. Michaelseni, M. striata paraissent presque complètement immobiles, les granulations cytoplasmiques se meuvent très lentement dans l'intérieur du corps sans affecter la forme extérieure ; il en est de même chez $R$. pilosa et chez $R$. porrecta. D'autres espèces sont beaucoup plus agiles, telle M. lumbrici. Le mouvement en sablier caractéristique de cette espèce, avec course très rapide du cytoplasme d'un pôle à l'autre avait frappé vivement les premiers observateurs qui ont étudié ce parasite. Chez M.agilis, ce mouvement s'observe également, mais nous avons vu que là, il ne se produit plus en ligne droite; en outre, il paraît être très capricieux, certains individus présentant un mouvement très rapide, d'autres paraissant complètement immobiles. On retrouve encore ce mouvement en sablier chez $M$. turbo et chez St. coronata ; mais il est un peu plus lent que chez les deux espèces précédentes. Ce mouvement se produit par ondes qui déforment généralement l'aspect extérieur du corps, l'allongeant, le raccourcissant, et amenant la formation de renflements et d'étranglements successifs qui peuvent donner à un parasite allongé comme $N$. magna un aspect moniliforme assez curieux; parfois le parasite ne se déforme point, c'est ce qui a lieu chez $N$. anguillula dont la cuticule est plus résistante et sans doute moins élastique. Ce parasite paraît plus rigide, les mouvements de 
va-et-vient du cytoplasme dans l'intérieur sont accompagnés de mouvements de torsion, qui rappellent beaucoup, comme nous l'avons vu, ceux des Nématodes. Ces mouvements de torsion se retrouvent du reste également chez toutes les espèces du genre Nematocystis.

Epimérite. - Chez la plupart des Monocystidées des Oligochètes, l'orientation du corps est nettement indiquée par l'allongement plus ou moins marqué de l'axe antéro-postérieur, ou par la direction des stries cuticulaires et des bandes de sarcocyte; mais il existe en outre dans un grand nombre d'espèces une différenciation du pôle antérieur en un véritable épimérite, parfois rudimentaire, et qui représente le rostre un peu accru du sporozoïte (mucron hyaloplasmique de beaucoup d'espèces, genou ou pied de $M$. agilis) ; mais parfois aussi très complexe et rappelant tout à fait celui des Polycystidées : tels sont ceux qui caractérisent les genres Rhynchocystis (trompe conique plus ou moins allongée) et Stomatophora (ventouse). Transitoire parfois, comme l'épimérite des Polycystidées, cet appareil peut d'autrefois persister pendant l'accouplement et ne disparaître que lors de la sécrétion de l'enveloppe kystique lorsque les parasites se sont comprimés mutuellement par la zone de contact; en outre, sa structure histologique est différente, ainsi, chez $R$. pilosa, il renferme et du sarcocyte et des fibrilles myocytiques que l'on ne rencontre jamais dans les épimérites de Polycystidées [LÉGER (1892).]

\section{Cytologie.}

Epicyte. - Sauf M. Michaelseni, toutes les espèces nous ont présenté des ornementations épicytaires variées : stries, sillons, verrues, poils. Lies stries et les sillons ont une direction longitudinale de même que chez toutes les Grégarines; parfois ils paraissent spiralés, comme chez $M$. striata et $P l$. Cuenoti, ceci provient sans doute d'une torsion du corps du parasite; 
les sillons séparent de larges bandes d'ectoplasme plus épais qui, se prolongeant postérieurement constituent une touffe de poils postérieurs chez $M$. lumbrici, $M$. striata, Z. cometa. Deux espèces sont remarquables en ce qu'elles présentent également des ornementations transversales : chez $N$. anguillula ce sont des stries circulaires sortes de petites crêtes peu accentuées, chez $M$. Cognettii ce sont au contraire des sillons de même profondeur et de même largeur que les sillons longitudinaux. Dans cette dernière espèce, l'ectoplasme n'est donc pas divisé en bandes longitudinales, mais en une série de carrés qui ornent la surface du corps d'une sorte de mosaïque. Au centre de ces carrés s'élèvent des perles arrondies qui peuvent être considérées comme des poils rudimentaires et nous donner par là la signification des productions piliformes si variées de $R$. pilosa, M. hirsuta, M.villosa, Z. pilosa, etc.

La "Gallertschichte » de Schewiakoff (1894) paraît manquer chez nos Monocytis; nous n'avons trouvé quelque chose de comparable à cette production que chez de très rares individus de $M$. agilis.

Sarcocyte. - Le sarcocyte est en général bien développé, particulièrement dans la région antérieure où il concourt chez quelques espèces à la formation de l'épimérite ( $R$. pilosa, $R$. porrecta, etc.).

Мroстте. - Le myocyte n'est pas toujours visible; mais, chez les espèces très agiles, on le met facilement en évidence par des colorations appropriées; il est hautement différencié et très contractile chez $N$. vermicularis, $N$. lumbricoides, $N$. magna, St. coronata et surtout chez M. lumbrici et $N$. anguillula. Quelques espèces nous ont montré à la fois des fibrilles longitudinales et des fibrilles transversales ( $M$. lumbrici, M.Elmassiani, etc., etc.).

Enтосуте. - L'endoplasme présente un assez grand nombre de caractères communs chez les diverses espèces; il diffère cependant par les dimensions des mailles du spongioplasme qui sont en rapport avec celles des grains de paramylon, et par la forme des inclusions chromatoïdes. 
Le spongioplasme renferme deux sortes de granulations, les unes très fines, colorables par l'Eosine, l'Orange, le Vert lumière; d'autres un peu plus volumineuses se colorent par l'Hématoxyline et ne diffèrent guère des inclusions chromatoïdes que par un volume moindre et par une forme toujours voisine de la sphère. Les inclusions chromatoïdes, sphériques chez $M$. agilis, sont en fer de lance chez $M$. lumbrici et irrégulières chez $S t$. coronata; chez $R$. pilosa, il en existe constamment une très volumineuse au voisinage de la queue. Ces inclusions sont logées dans les mailles de spongioplasme, on n'en rencontre pas dans l'hyaloplasme.

Parfois, on observe encore dans l'endoplasme des masses chromidiales très volumineuses comme celles que nous avons signalées chez $M$. agilis; ou des granulations nutritives? (région antérieure de $S t$. coronata, entre ventouse et noyau; épimérite de $R$. pilosa et $R$. porrecta).

Dans les stades jeunes, nous avons noté presque partout un spongioplasme éosinophile; il devient au contraire orangophile chez les adultes. Le Mann le colore en bleu pâle au début, en violet clair et en rouge dans les stades ultérieurs.

Noyau. - La structure du noyau s'est montrée assez variable chez les diverses espèces. On peut noter cependant qu'il existe partout un appareil karyosomien central plus ou moins complexe dans lequel s'est réfugiée totalement ou en partie seulement la chromatine nucléaire, le reste de celle-ci pouvant être dispersé en grains fins sur un réseau lininien.

Karyosome. - Le karyosome peut être unique, ou fragmenté en plusieurs morceaux de volumes divers. Il y a un seul karyosome volumineux chez $M$. agilis, M.hirsuta, M. Michaelseni, M. villosa, M. Cognettii, N. vermicularis et N. magna, et plusieurs karyosomes chez M. lumbrici, M. striata, M.crenulata, M. Elmassiani et $\mathrm{Pl}$. Cuenoti. On en observe un, rarement plusieurs chez $R$. pilosa, $R$. porrecta, St. coronata, et $N$. lumbricoides; au contraire il y en a plusieurs, et rarement un seul chez N. anguillula. 
Le plus souvent, tous les karyosomes offrent la même structure; mais il y a parfois aussi deux espèces de karyosomes, comme nous l'avons vu chez $M$. Le Mêmei et $M$. lumbrici olidi, mais qui semblent provenir l'un de l'autre par une sorte de bourgeonnement.

En général, le karyosome est vacuolaire, et il présente à sa surface une croûte dont la coloration diffère de celle de la moelle; avec l'Hématoxyline, elle prend simplement une teinte un peu plus foncée; mais le Mann la colore en violet, tandis qu'il teint le reste du karyosome en rouge. Il peut arriver que le karyosome offre une structure beaucoup plus compliquée, comme celle que nous avons décrite chez Pl.Cuenoti. Même chez les espèces où il est en général simple, il se présente parfois avec un aspect plus complexe : chez $M$. agitis par exemple, il apparaît à certains moments comme divisé en deux parties de volume à peu près semblable, mais dont l'une est colorée par le Mann en violet, tandis que l'autre se colore en rouge, ce qui rappelle le double karyosome de $M$. Le Mêmei et de M. lumbrici olidi. Du karyosome paraissent provenir par bourgeonnement une grande quantité de grains chromatiques de volume variable qui peuvent demeurer accolés à sa surface ou lui être longtemps rattachés par des pédicelles de longueur variable. Nous l'avons vu ainsi, chez $M$. agilis, envoyer jusque dans le cytoplasme de longues traînées arborescentes de fines granulations chromatiques.

Durant tout le cours de la vie végétative, le karyosome semble être le siège de nombreuses métamorphoses et jouer un rôle important dans les manifestations vitales du noyau, et ceci est bien conforme aux vues des auteurs qui l'ont étudié chez d'autres parasites, entre autres Sieduecki (1905) MorofF (1907) LÉger et Dubosce (1908). Ce n'est pas ici le lieu de revenir sur les innombrables discussions auxquelles il a donné naissance.

Kystes et Sporocystes. - Nous n'avons pas suivi en détail les phénomènes sexuels qui se déroulent dans les kystes et qui 
sont bien connus chez les Monocystis du Lombric depuis les recherches de Brasil (1905 b), Hoffirann (1909) et sur lesquels LÉGER et DuboscQ ont dernièrement encore (1909 b) jeté quelques éclaircissements.

Dans la description que nous avons donnée des divers parasites, nous n'avons pu indiquer les dimensions de beaucoup de sporocystes parce qu'un grand nombre d'espèces se rencontrant en même temps dans le même hôte, il est impossible de savoir à laquelle d'entre elles appartiennent les kystes et les sporocystes que l'on rencontre. Les anciens observateurs, SchNeIder (1875) en particulier, avaient décrit pour une même Grégarine des macrospores et des microspores; CúnNo'T (1901) et BRASIL (1905 b) considèrent ces formes comme appartenant à des espèces différentes, BRASIL est très affirmatif à ce point de vue: "Je pense, dit-il, que tout le monde est d'accord pour attribuer la valeur d'un caractère spécifique à ces mesures » (celles des sporocystes) " et qu'il n'entre plus dans les idées de personne de donner à un seul Monocystis des kystes à macrospores et des kystes à microspores ". Qu'il me permette cependant de n'être point de son avis et de me ranger à l'opinion de SchneIder. Sans doute il est difficile d'élucider la question pour la plupart des espèces, ainsi que nous l'avons vu plus haut; cependant, dans ces cas déjà, alors que j'observais seulement dans un hôte, trois ou quatre espèces de Monocystidées bien distinctes par leurs caractères végétatifs, les sporocystes me présentaient un bien plus grand nombre de variétés de taille. Mais le fait n'est plus douteux lorsqu'on s'adresse à des espèces très volumineuses, remarquables par conséquent par le volume considérable de leurs kystes qui ne peuvent être confondues avec ceux des autres espèces et dans ce cas nous avons trouvé toujours des macrospores et des microspores; nous avons du reste indiqué les dimensions respectives de ces spores à propos de $M$. herculea chez Octolasium lacteum.

Nous n'avons malheureusement observé aucun fait qui puisse 
nous renseigner sur la signification de cette double forme de sporocystes.

\section{Modes de dissémination.}

Les kystes remplis de sporocystes et les sporocystes libres s'accumulent peu à peu dans les vésicules séminales où ils constituent des amas parfois très considérables entourés par les phagocytes qui finissent, à la longue, par en détruire une notable quantité.

Comment les sporocystes gagnent-ils l'extérieur pour propager le parasite chez de nouveaux hôtes ? Cette propagation a lieu d'une façon intensive, au moins chez certaines espèces, puisque nous avons vu qu'il nous avait été impossible de nous procurer un seul exemplaire adulte de Lumbricus ou de Pheretima qui soit absolument indemne de parasites.

Les anciens auteurs croyaient que les sporocystes s'ouvraient directement dans les vésicules séminales elles-mêmes. Ainsi Lieberkühn (1855), Gabriel (1876-1879), Ruschiaupt (1881) supposent que les spores se transforment directement en Grégarines dans le corps même de l'hôte où elles sont formées. On trouve représentés dans Ruschinupt (taf. xxiI, fig. 31 , $32,34,35$ ) tous les stades de la pénétration de ces sporocystes dans une "Spermamutterzelle " et la série des métamorphoses qui les transforment en jeunes Grégarines. RuschHAUPT avait du reste des idées tout à fait erronées sur le contenu du sporocyste, ainsi il se refusait à donner aux sporozoïtes la valeur de vrais germes; pour lui, le reliquat sporal représentait seul dans le sporocyste la jeune Grégarine.

On sait aujourd'hui d'une façon indubitable que les sporozoïtes sont bien les germes de la Grégarine; en outre, toutes les recherches sur le développement de ces parasites nous ont appris que l'action des sucs digestifs de l'hôte était indispensable pour provoquer la déhiscence des sporocystes (Cf. notamment LÉGer et DuboscQ (1902 et 1904). Bütschli (1882) 
du reste, avait montré déjà combien paraissait invraisemblable cette germination sur place, étant donné la quantité colossale de kystes que renferment les testicules d'un Ver de terre et le nombre relativement beaucoup moins considérable de Grégarines à l'état végétatif que l'on y rencontre.

De nos jours, on admet généralement que le passage du sporocyste par l'intestin de l'hôte est absolument nécessaire pour amener l'ouverture des valves. Cependant, Drzewecki (1907 b) croit avoir vu des sporozoïtes de Stomatophora coronata nager dans le kyste, en sortir et se répandre dans les vésicules séminales de Pheretima, où ils prendraient la forme de cellules arrondies ou ovoïdes représentées (fig. 1 à 3). Nous croyons qu'il s'agit là d'une erreur d'interprétation.

Schmid (1854) pensait que les spores étaient expulsées avec le sperme de l'hôte au moment de la copulation. Il est possible que ce soit là en effet un des modes empruntés par les sporocystes pour gagner l'extérieur. Cependant, nous n'avons jamais trouvé de kystes, ni même de sporocystes isolés, ni dans les pavillons ciliés qui servent de réservoir aux spermatozoïdes mûrs, avant la copulation, ni dans les canaux déférents, ni dans les réservoirs séminaux où le sperme est emmagasiné après la copulation.

Si ce mode d'évacuation existe, il serait donc purement accidentel. Nous n'avons pas vu non plus de kystes et de sporocystes en train d'émigrer des vésicules séminales vers la cavité générale. On observe cependant dans celle-ci de nombreux kystes grégariniens. Ces kystes sont souvent très abondants dans les segments terminaux, où on les rencontre enveloppés dans de volumineux amas phagocytaires avec des Nématodes englobés vivants parfois, ainsi que j'ai pu le constater souvent chez Octolasium lacteum Örley et des soies de l'hôte, ou d'autres corps inertes. Cette accumulation dans la région postérieure serait due, d'après METCHNIKoFF (1892) et CUÉnot (1898) aux simples mouvements du corps de l'hôte.

De tels kystes appartiennent sans doute à des Grégarines 
cœlomiques, ils sont en effet abondants surtout chez Octolasium lacteum Örley et chez Pheretima hawayana Rosa où les formes cœlomiques sont communes; peut-être aussi, s'agit-il de kystes de parasites des pavillons ciliés : N. magna et $P l$. Cuenoti par exemple, comme portent à le croire les dimensions considérables qu'ils présentent chez $L$. herculeus Sav. et chez $H$. longus Ude et $H$. caliginosus Sav.

On trouve aussi dans le cœlome des sporocystes isolés, englobés ou non par les phagocytes.

Ces sporocystes sont amenés au dehors, soit par la nécrose des segments terminaux, soit par leur séparation accidentelle du reste du corps, faits qui paraissent se produire assez fréquemment. En outre, j'ai pu constater souvent que, chez certains Helodrilus, et surtout chez les Pheretima, ces sporocystes sont émis en grande quantité par les pores dorsaux en même temps que les éléocytes, dont le mécanisme de rejet a été étudié par Cú́not (1898).

En ce qui concerne la mise en liberté des sporocystes enfermés dans les vésicules séminales, nous ne voyons guère qu'un seul mode possible, la mort de l'hôte. Les tissus du Ver mort se corrompent et les sporocystes très résistants restent intacts ; ils sont ensuite absorbées par d'autres Vers dans l'intestin desquels ils s'ouvrent pour livrer passage aux germes. Il faut remarquer en outre que les Lombrics sont mangés par une foule d'animaux carnassiers tant Vertébrés qu'Invertébrés : Mammifères, Oiseaux, Reptiles, Batraciens, Insectes, Arachnides, Myriapodes. Les spores de Monocystis absorbées avec le corps de leur hôte ressortent intactes avec les excréments du carnassier qui les a dévorées; elles peuvent alors être reprises par les Vers de terre, s'ouvrir dans leur intestin et donner passage aux sporozoïtes. Dès $\mathbf{1 8 4 5}$, DuJARdiN (qui comparait les spores des Grégarines du Lombric dont il ignorait la véritable nature aux "psorospermies » de Müller) observait des spores intactes dans l'intestin des Taupes qui se nourrissent de Vers de terre. Plus tard, Pfeiffer (1891) a remarqué 
également que les spores des Monocystis traversent sans subir aucune altération l'intestin des Bécasses, LÉGER (1899) a fait la même observation pour l'Orvet, et aussi (observations inédites) pour les Phalangides. Nous avons trouvé également des sporocystes mûrs de Monocystis, en parfait état dans les excréments de Carabes nourris avec des Vers de terre. Toutes ces spores sont susceptibles de s'ouvrir lorsqu'elles sont absorbées par des Lombrics et elles livrent passage à des sporozoïtes vivants, comme nous avons pu l'observer. C'est donc là un mode de propagation naturel du parasite et, sans doute, des plus actifs.

\section{Développement.}

Nous avons décrit précédemment, pour quelques espèces du moins, $M$. agilis et $S t$. coronata par exemple, tous les stades de leur développement dans les vésicules séminales depuis l'époque de leur pénétration dans le follicule spermatique jusqu'à la formation des sporocystes. Quelques-uns de ces stades avaient été remarqués depuis longtemps par SснміDт (1854) et par RusCHHAUPT (1881). GABRIEL (1897) et plus récemment Drzewecki (1907 $b$ ) auraient même vu des sporozoïtes libres dans le liquide cœlomique et dans les testicules. Nous avons dit déjà ce qu'il faut penser des idées du dernier observateur qui aurait vu les sporozoïtes se mouvoir dans un kyste, à l'intérieur des vésicules séminales. Pour notre part, il ne nous a pas été possible d'observer des sporozoïtes libres dans le fluide séminal, avant l'instant où ils pénètrent dans les spermatosphères. Nous ne savons done pas comment ils arrivent dans les vésicules séminales. Mais des expériences précises nous ont permis d'observer la déhiscence des sporocystes dans l'intestin du Lombric. Nous avons ouvert un nombre considérable de Lombrics récoltés au dehors sans arriver à trouver de sporocystes dans leur intestin. Les observations de ce genre sont du reste très difficiles à cause du contenu intes- 
tinal de ces animaux, lequel, formé en grande partie de petits grains de sable ou de particules organiques grossières ne se prête pas bien à un examen microscopique attentif. Force nous a done été d'avoir recours aux infections artificielles qui ont si bien réussi à LÉGer et DUBOSCQ (1902-1904) pour les Polycystidées des Arthropodes. Malheureusement nous avions affaire à un matériel peu commode. Le Ver de terre ne mange pas volontiers la pâtée qu'on lui offre, lorsque nous en placions dans un tube avec une masse de débris organiques ou de marc de café finement pulvérisés dans laquelle nous avions incorporé force sporocystes, ils ne touchaient absolument pas à cette substance et se laissaient plutôt mourir à côté. Il eut fallu peut-être préparer une masse considérable de substance ainsi malaxée, mais alors la faible quantité de sporocystes dont nous pouvions disposer, disséminée dans une grande quantité de substance inerte aurait passé inaperçue dans l'intestin de nos sujets. Du reste, même si quelques sporocystes avaient été absorbés de cette façon, nous eussions manqué de données précises sur le moment de leur injection. Nous avons alors essayé d'injecter dans l'intestin de nos Vers une bouillie de sporocystes recueillie dans les testicules de leurs congénères. C'est cette méthode, malgré les difficultés qu'elle présente qui nous a donné quelques résultats.

On ouvre au hasard une certaine quantité de Vers adultes et on dilacère sur lame le contenu de leurs vésicules séminales, on choisit les points où se trouvent en abondance des sporocystes mûrs que l'on fait pénétrer dans une canule, puis on introduit le mélange dans l'intestin d'un sujet que l'on a fait préalablement jeûner pour débarrasser son intestin des pierrailles qu'il contient. Neuf fois sur dix le Ver rejette la mixture qu'on lui introduit ainsi dans le pharynx; ou bien son intestin se perfore et les sporocystes sont poussés dans le cœlome. Avec un peu d'habitude, on arrive cependant à envoyer dans l'œsophage quelques sporocystes. Si l'on examine les excréments de l'Oligochète une dizaine d'heures après l'expérience, on y ren- 
contre quelques sporocystes vides. Ceux-ci sont représentés. (fig. XcIv), on voit que les deux pointes du fuseau ont disparu, laissant à chaque extrémité un goulot étroit par lequel se sont échappés les sporozoïtes. La surface interne de l'exospore se présente comme une ligne plus gmbrée, la surface externe de cette exospore de même que la surface interne de l'endospore sont marquées par des lignes plus minces. L'endospore est en général plissée et dans ses plis restent parfois

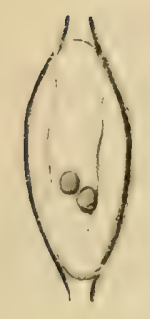

XCIV

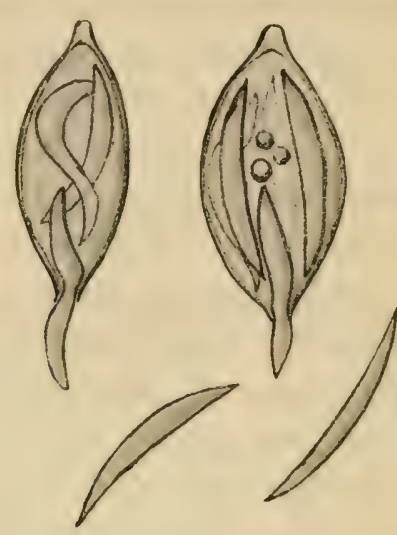

$X C V$

FIG. XCIV.Sporocyste vide trouvé dans les excréments de Lumbricus $\times 1.300$. Les sporozoïtes se sont échappés par les orifices polaires. Les grains de reliquat ont été retenus dans les plis de l'endospore. - FIG. XCV. Dehiscence du sporocyste dans l'intestin du Lombric. Sortie des sporozoïtes $\times 1.300$.

accrochés les grains de reliquat qui n'ont pas suivi les sporozoïtes dans leur migration.

J'ai rencontré souvent ainsi des sporocystes ouverts sans arriver à surprendre la sortie des sporozoïtes. Enfin favorisé par le hasard, j'ai pu assister à cette sortie. Un Lombric ouvert une heure et quart après l'injection d'une certaine quantité de sporocystes me montra de nombreuses spores dans le pharynx et quelques-unes dans le gésier. Aucune n'était ouverte, aucune même ne présentait la moindre trace d'altération. Quelquesunes parvenues au début de l'intestin moyen étaient déjà 
vides, l'une d'elles présentait seulement une ouverture à l'une de ses extrémités et les sporozoïtes étaient en train de s'échapper. Ces corpuscules très clairs, d'aspect hyalin, réfringent, présentaient dans l'intérieur du sporocyste des mouvements serpentiformes; arrivés en face du goulot ouvert, ils s'y engageaient un à un par une extrémité et s'échappaient ainsi successivement avec effort, car ils sont plus larges que l'orifice et sont ainsi comprimés pour arriver à gagner l'extérieur. A mesure qu'ils s'échappent, la spore semble se dégonfler, elle possède sans doute une certaine élasticité et, en comprimant les sporozoïtes restés à l'intérieur, doit faciliter leur expulsion (fig. XCV.)

J'ai cherché à retrouver ces sporozoïtes dans des coupes de l'intestin pour suivre leur migration à travers sesparois; malgré les expériences multipliées que j'ai faites et le grand nombre de coupes examinées, je n'ai rien retrouvé ; sans doute les sporozoïtes ainsi introduits sont trop peu nombreux et la surface intestinale sur laquelle ils se disséminent trop grande pour que l'on ait quelque chance de suivre leur passage dans le colome. Quelque incomplets que soient ces résultats, je pense qu'ils ne sont pas sans intérêt, car ils démontrent d'une façon péremptoire que les sporocystes des Monocystis s'ouvrent dans l'intestin des Lombrics et livrent ainsi passage à des sporozoïtes vivants et par suite que c'est certainement par la voie intestinale que se fait l'infection.

\section{Spécificité parasitaire.}

Si cette méthode d'infection artificielle était plus pratique, il eut été intéressant de lui demander quelques renseignements sur la spécificité parasitaire de nos Monocystidées, malheureusement elle est d'un maniement trop peu commode pour donner des résultats satisfaisants. Nous ne pouvons done donner sur ce point que les résultats qui nous ont été fournis par l'obser- 
vation directe des faits. La spécificité parasitaire est généralement admise pour les Grégarines, elle semble exister chez tous les Arthropodes ; pour les Myriapodes de la Corse et du Continent, en particulier, les recherches de LÉGER et DuBoscQ (1903) ont montré qu'elle était rigoureuse. Chez les Oligochètes, nous retrouvons quelques exemples d'une semblable spécificité : ainsi $M$. striata semble propre à Lumbricus terrestris L. Müll.; il en est de même de $N$. magna; $M$. hirsuta ne se trouve que chez $L$. castaneus Sav., $N$. vermicularis se rencontre seulement chez Helodrilus longus Ude et $N$. lumbricoides chez les Helodrilus caliginosus du continent; mais à côté de ces parasites qui semblent propres à une espèce donnée d'Oligochète, il en est d'autres que l'on rencontre indifféremment dans toutes les espèces du genre Lumbricus, ou dans toutes les espèces d'Helodrilus ou de Pheretima; quelques-unes même semblent exister à la fois chez les Lumbricus et les Helodrilus.

\section{Distribution géographique.}

La distribution géographique des Monocystidées des Oligochètes nous montre quelques faits intéressants : à côté d'espèces excessivement répandues, il en est d'autres qui semblent localisées à certaines régions. Nous avons vu précédemment que Brastl $(1905 b)$ semblait n'avoir point observé $M$. lumbrici Henle dans les L. herculeus de Normandie, alors que le parasite est abondant en Lorraine (CúÉNoT), en Franche-Comté en Dauphiné et en Provence; Cú́not (1901) au contraire n'aurait pas trouvé en Lorraine $M$. agilis étudiée en Normandie par Brasil et qui est si commune dans l'Est de la France depuis Epinal jusqu'à Toulon. D'autres Grégarines donnent lieu à des observations semblables ainsi les Helodrilus caliginosus d'ElKantara hébergent M. Le Mêmei que je n'ai jamais rencontrée dans les mêmes hôtes habitant en France; $H$. choloroticus donne asile en Franche-Comté à Z. Legeri, le même hôte aux envi- 
rons de Grenoble ne renferme pas de parasites, et cependant dans les échantillons recueillis dans les serres du Jardin des Plantes de la ville et qui sont probablement d'origine exotique, on trouve une Grégarine $M$. Cognettii très différente de $Z$. Legeri.

Nos documents sont trop incomplets pour qu'on en puisse tirer des conclusions générales, mais ils montrent combien il serait intéressant d'étudier à ce point de vue les diverses espèces d'Oligochètes cosmopolites sur des échantillons recueillis en diverses stations du globe. Les résultats d'une telle étude pourraient nous donner des renseignements précieux sur l'origine des espèces parasitaires, question encore si obscure de la biologie générale.

Relations entre le développement des parasites et celui des glandes sexuelles de l'hôte.

Pour terminer cette étude comparative des diverses Monocystidées, il nous reste quelques mots à dire sur les rapports qui existent entre le développement de ces Grégarines et la marche des saisons.

Ruschiaupt (1885) et Pfeiffer (1891) avaient remarqué qu'il existe une certaine corrélation entre les divers stades du développement des Monocystis du Lombric et les saisons, ou ce qui revient au même, le développement des cellules sexuelles de l'hôte.

Pfeiffer a noté qu'en hiver, à Weimar, N. magna prédomine; en été au contraire, ce serait $M$. agilis. Je n'ai rien observé de semblable; il est vrai que, ainsi que nous l'avons vu PferfFER confond sans doute $N$. magna et $R$. pilosa ; j'ai remarqué en effet que $R$. pilosa paraît plus abondante de novembre à avril, sans doute parce que son sporozoïte peut pénétrer dans des follicules spermatiques déjà très avancés dans leur évolution, ce qui est impossible aux sporozoïtes de $M$. agilis par exemple.

ARCH, DE ZOOL, EXP. EY GEN, - 5 SERIE, - T, III. - (II). 
Quant aux autres indications de Pfeiffer et à celles de RuschHaupt, elles concordent en général avec nos propres observations, en ce qui concerne du moins les Lombrics adultes, pourvus d'un clitellum bien développé. Nous ferons observer à ce sujet qu'il ne faut pas accorder à ces considérations une importance trop considérable, car elles n'ont qu'une valeur très relative, variable avec les stations, avec la température, avec l'âge de l'hôte. Ainsi, les Lombrics jeunes, encore dépourvus de clitellum, à glandes génitales peu développées, renferment en toute saison des Grégarines à tous les stades, surtout de nombreuses Grégarines jeunes intrablastophoriennes, et il faut considérer sans nul doute ce fait comme étant en rapport avec l'état des glandes sexuelles immatures et constamment en travail de préspermatogenèse.

Ce développement des parasites est commandé par le développement même des cellules qui servent à les nourrir. Chez les Pheretima, on observe en tout temps dans les vésicules séminales des follicules spermatiques à tous les stades du développement et, comme conséquence on trouve aussi à chaque instant des Grégarines à tous les stades. Chez les autres Oligochètes, les stades végétatifs des parasites sont surtout abondants au' moment des poussées sexuelles; chez les Lumbriculus variegatus $\mathrm{O}$. Müll, par exemple, on ne trouve des $M$. Duboscqi que de novembre à mai, en dehors de cette époque, on n'en observe plus; sans doute cette espèce commence son développement dans les glandes sexuelles avant de devenir cœlomique.

\section{CHAPITRE IV}

\section{ALTÉRATIONS ET PARASITES}

Dégénérescence.

Nous avons énuméré en étudiant le développement des Monocystidées les transformations curieuses présentées par le noyau de quelques espèces sur la fin de leur vie végétative; 
nous avons vu que, chez $M$. agilis et $S t$. coronata en particulier, la membrane nucléaire s'évanouit parfois complètement pour laisser passer librement la chromatine du noyau dans le cytoplasme.Quelle que soit la signification de cette sorte de crise: " épuration nucléaire ", " bain de régénérescence " ou émission rapide de grains de ferments destinés à transformer les réserves, nous avons vu qu'il convient de la considérer comme normale. Elle n'amène ni la dégénérescence, ni la mort du parasite; cette dégénérescence et cette mort sont produits assez souvent au contraire par une série de processus pathologiques sur lesquels il convient de dire quelques mots. Nous allons les étudier successivement chez les diverses espèces.

\section{Monocystis agilis.}

Certains exemplaires de $M$. agilis, au lieu de présenter la forme normale en fuseau dissymétrique irrégulier et allongé, se raccourcissent en s'arrondissant ; les deux pointes du fuseau se ratatinent et se recourbent en s'appliquant étroitement sur le corps du parasite, puis elles s'atrophient complètement et disparaissent (fig. 75).

La Grégarine est devenue un fuseau court, fortement renflé en son milieu et symétrique par rapport à son plan équatorial; elle a pris la forme d'un énorme sporocyste qui serait dépourvu d'enveloppe. Son grand axe continuant à se raccourcir tandis que le petit axe s'accroît, elle ne tarde pas à devenir sphérique et à dégénérer. Mais le plus souvent la dégénéres-cence commence avant que le parasite ait atteint cette forme et il devient la proie des phagocytes de l'hôte.

Dans une Grégarine ainsi en voie de dégénérescence, les phénomènes normaux étudiés dans les chapitres précédents se poursuivent pendant quelque temps; on observe par exemple la disparition de la membrane nucléaire et le passage de la chromatine dans le cytoplasme; mais le noyau disparaît complètement ici et ne se reforme pas. Les travées du spongioplasme sont bientôt remplies de ces petites boules chroma- 
toïdes entourées de vacuoles claires (fig. 75) que nous avons signalées déjà dans les parasites en train d'absorber leurs réserves. Les grains de paramylon peu à peu se gonflent démesurément pendant que leur vacuole centrale grossit et devient irrégulière (fig. 74). Ils sont très colorables au début par le Rouge Magenta ou l'Orange, puis cette colorabilité diminue et les grains fortement hypertrophiés restent à peu près incolores ; ils disparaissent ensuite, sans doute en se dissolvant dans le cytoplasme ambiant. Lorsqu'ils ont disparu, le cytoplasme se vacuolise et subit le plasmarrhexis.

Lorsque la chromatine du réseau nucléaire a été émise dans le cytoplasme, le karyosome semble se dissoudre dans le suc nucléaire qui devient très hyperchromatique (fig. 74). La colorabilité du suc nucléaire diminue ensuite peu à peu; il ne reste plus bientôt, à la place du noyau qu'une plage légèrement plus colorée que le cytoplasme environnant et qui disparaît à son tour.

Le parasite est englobé par les phagocytes de l'hôte avant la fin de ces processus dégénératifs; il se ratatine et bientôt il n'en reste d'autre trace qu'un amas de phagocytes bourrés de fragments cytoplasmiques ou fortement distendus par les réserves qu'ils ont absorbées et assimilées.

Les phagocytes peuvent attaquer la Grégarine dès le début des phénomènes que nous venons d'énumérer sans produire de changement notable dans la succession de ces phénomènes. On constate toujours une hyperchromasie du suc nucléaire avec disparition du karyosome et finalement évanouissement du noyau, puis une vacuolisation du cytoplasme suivie de plasmarrhexis.

Dans quelques cas très rares, une hypertrophie considérable du noyau et du karyosome précède les stades décrits précédemment.

Dans les phénomènes pathologiques que nous venons de signaler, la dégénérescence du parasite commence avant l'attaque des phagocytes et celle-ci semble n'être qu'une conséquence de cette dégénérescence primitive. 
Cette même corrélation existe sans doute aussi pour $M$. lumbrici où s'observent des altérations semblables, mais elle est moins manifeste; je n'ai jamais vu en effet de $M$. lumbrici en dégénérescence qui ne soit déjà entourée d'un manteau de phagocytes.

\section{M. lumbrici,}

De même que $\boldsymbol{M}$. agilis, $M$. lumbrici en dégénérescence commence par s'arrondir ; mais il y a en outre ici hypertrophie notable du parasite. Alors que, chez Lumbricus terrestris, les individus normaux de $M$. lumbrici les plus volumineux ne dépassent guère $200 \mu$ sur $60 \mu$, les Grégarines phagocytées ont toujours plus de $200 \mu$ sur $150 \mu$; nous en avons rencontré qui mesuraient $354 \mu$ sur $225 \mu$.

Le parasite recouvert déjà d'une mince conche de phagocytes continue cependant encore à se mouvoir, mais son mouvement est considérablement ralenti, de plus, il ne se produit plus en direction rectiligne ; les grains de réserve et les granulations cytoplasmiques se déplacent lentement en cercle, rappelant le mouvement normal de $M$. villosa. Ce mouvement cesse lorsque commencent à se montrer les premières traces d'altération dans le cytoplasme.

L'hypertrophie de la masse cytoplasmique n'amène pas celle du noyau qui reste peu volumineux. Dans des parasites normaux de $270 \mu$ sur $75 \mu$, le noyau atteint $42 \mu$ sur $12 \mu$; au contraire, chez des individus phagocytés de $345 \mu$ sur $225 \mu$, le noyau n'a pas plus de $21 \mu$ de diamètre. En général la forme du noyau est sphérique, tandis qu'il est ovoïde dans les parasites bien portants. Au lieu de renfermer un karyosome fragmenté, il ne présente jamais qu'un seul karyosome (fig. 76, 77); cependant on observe quelquefois des étranglements successifs qui rendent ce karyosome moniliforme; la fragmentation ne va pas plus loin; il y a là une sorte d'arrêt de développement qui nous donne un renseignement important sur l'origine et le mode de formation du karyosome multiple des individus sains (fig. 181). 
Les phagocytes ne forment pas toujours une couche continue autour de la Grégarine ; ils paraissent d'abord être épars sur sa surface; puis, leur nombre augmente, ils enserrent étroitement le parasite, et chacun d'eux finit par être logé dans une dépression du corps de ce parasite (fig. 76). Cette dépression s'accentue de plus en plus et la Grégarine apparaît finalement découpée en lobes irréguliers séparés par des cavités plus ou moins profondes. Ces lobes peuvent même se détacher du reste du corps et être entourés complètement par les phagocytes. On trouve fréquemment des fragments de Grégarine dépourvus de noyaux phagocytés, et qui ont vraisemblablement été détachés du corps par ce processus.

Les cavités où se logent ainsi les phagocytes ne paraissent pas être creusées par ceux-ci à la suite d'un processus de digestion, car la cuticule demeure intacte en ces points comme sur le reste du corps. Mais au fond de ces cavités, l'ectoplasme du parasite présente des côtes saillantes, sorte de replis de l'ectoplasme, tout à fait semblables à celles que l'on observe dans les Grégarines normales chez lesquelles il y a eu de fortes contractions du myocyte. Les cavités seraient donc dues à une contraction des fibrilles du myocyte, ce serait une sorte de réaction de la Grégarine contre l'action du phagocyte.

Les Grégarines ainsi phagocytées semblent rester longtemps sans présenter d'autres phénomènes que les divers changements de forme et de dimensions que nous venons d'indiquer. Puis apparaissent des altérations tout à fait comparables à celles qui ont été décrites plus haut pour $M$. agilis.

L'ectoplasme diminue d'épaisseur, il y a gonflement puis dissolution des grains de paramylon, vacuolisation du cytoplasme et enfin plasmarrhexis et ratatinement du parasite.

Dans le noyau, le karyosome resté unique est toujours assez volumineux, il est placé au centre et entouré par une couche de granulations chromatiques (fig. 77); d'autres granulations de même nature forment des travées rayonnantes qui vont rejoindre la membrane nucléaire. Puis le karyosome semble se dissou- 
dre dans le suc nucléaire qui devient très colorable, en même temps que la membrane perd son individualité et que l'ensemble du noyau prend une forme irrégulière. La colorabilité de la plage nucléaire diminue de plus en plus et finalement toute trace du noyau a disparu. Ce sont bien là les phénomènes déjà constatés chez $M$. agilis. Les derniers stades de la dégénérescence sont tout à fait identiques et l'on a encore comme terme ultime la formation d'un nodule phagocytaire au centre duquel il ne reste même plus trace du parasite.

Dans les fragments dépourvus de noyau, l'altération du cytoplasme se produit absolument de la même façon que pour les parasites nucléés.

Il arrive souvent que ces parasites phagocytés, ainsi que les fragments qui s'en détachent sont attaqués par des Bactéries qui hâtent la dégénérescence comme nous le verrons plus loin.

\section{R. pilosa.}

On observe chez $R$. pilosa comme chez les espèces précédentes des phénomènes particuliers de dégénérescence, ils diffèrent un peu de ceux que nous venons de décrire.

Nous avons $v u$, en étudiant les parasites normaux qu'il leur arrive souvent de se fragmenter à cause de la fragilité de leur cuticule.

Les fragments ainsi séparés peuvent être saisis de suite par $\mathrm{l}_{\mathrm{es}}$ phagocytes; les phénomènes consécutifs sont alors identiques à ceux qui se produisent dans le même cas chez $M$. lumbrici; les boules mucoïdes chromatophiles qui se forment dans le cytoplasme pendant la disparition des grains de paramylon sont cependant plus nombreuses et beaucoup plus volumineuses.

Il arrive aussi que ces fragments échappent à la phagocytose, ils subissent alors la dégénérescence granuleuse que nous retrouverons chez $M$. agilis et $M$. lumbrici attaquées par des Bactéries. Les travées du spongioplasme se remplissent de grains fortement colorés par l'Hématoxyline, les grains de 
paramylon disparaissent et leur place est bientôt comblée par les granulations colorées (fig. 79).

Certains parasites subissent la dégénérescence décrite pour M. agilis : disparition des grains de paramylon et formation de boules chromatoïdes envacuolées, dont l'aspect rappelle les boules mucoïdes observées par LÉGER et DuBoscQ (1902) dans les cellules de l'épithélium intestinal de divers Arthropodes; dissolution du karyosome dans le suc nucléaire et hyperchromatie de celui-ci, puis imprégnation du cytoplasme de la trompe par cette chromatine dissoute (fig. 78) ; enfin disparition complète du noyau; vacuolisation du cytoplasme et plasmarrhexis.

Dans ces $R$. pilosa en dégénérescence, on observe parfois de légères constrictions de l'épicyte assez régulièrement disposées, qui rendent métamérique le corps du parasite (fig. 88). $\mathrm{Au}$ niveau de ces contrictions de la cuticule qui ne sont jamais très accentuées, on voit la couche de sarcocyte s'épaissir suivant une ligne circulaire et s'enfoncer dans l'endoplasme, constituant une sorte de diaphragme annulaire le plus souvent incomplet, mais pouvant aussi devenir une cloison continue. Le corps du parasite est ainsi segmenté en tronçons de longueur très inégale et son aspect rappelle celui que LÉGER (1906) a décrit chez Taeniocystis mira; seulement ici les cloisons sont constituées par du sarcocyte, comme la cloison normale qui sépare le protomérite du deutomérite, chez les Polycystidées et non par des grains d'excrétion comme chez le parasite des larves de Ceratopogon. C'est sans nul doute selon ces cloisons que se séparent les fragments de $R$. pilosa qui se détachent du corps maternel.

Ce cloisonnement semble bien dû ici à une action mécanique pure et non à des phénomènes trophiques puisqu'il se produit chez des parasites en dégénérescence chez lesquels la nutrition ne se fait plus sans aucun doute. 


\section{N. vermicularis.}

En décrivant $N$. vermicularis, nous avons vu que tous les exemplaires jeunes de ce parasite rencontrés dans les vésicules séminales de $H$. longus sont recouverts de phagocytes de l'hôte; ceux-ci forment autour de la Grégarine un manteau continu beaucoup plus épais vers les deux extrémités. Le nombre de ces phagocytes n'augmente pas ou augmente très peu pendant la croissance du parasite, de sorte que, lorsque ce parasite a grossi, les phagocystes ne sont plus disposés à sa surface en une couche continue, mais épars ça et là, souvent assez éloignés les uns des autres.

La Grégarine ne semble nullement souffrir de la présence des phagocytes, son développement n'est pas arrêté. Vers les extrémités du corps où les phagocytes insinués entre les expansions piliformes du parasite forment souvent une couche très épaisse, il se produit quelquefois un amincissement du diamètre de la Grégarine de sorte que celle-ci paraît pourvue à l'une des ses extrémités ou à ses deux extrémités d'une trompe parfois assez allongée renfermée dans un épais manchon phagocytaire. Il peut même arriver que la masse de phagocytes produise en un point un étranglement si accentué que la trompe se sépare du reste du corps. Celui-ci ne paraît pas s'altérer à la suite de cette mutilation; quant au fragment amputé il dégénère et est absorbé par les phagocytes qui l'entourent.

\section{St. diadema.}

Cet englobement par les phagocytes, sans action nuisible pour le parasite se retrouve chez $S t$. diadema. Cette Grégarine est en effet presque constamment enfermée dans un épais massif cellulaire qui semble constitué par des phagocytes, et elle vit ainsi sans présenter de phénomènes de dégénérescence ; on constate simplement une hypertrophie assez notable du corps et la formation de lobes profondément découpés, analogues à ceux que nous avons signalés chez les $M$. lumbrici 
phagocytées. Nous n'avons jamais remarqué d'autres altérations consécutives.

Les Grégarines colomiques connues jusqu'ici échappent en général aux phagocytes, quelques individus seulement sont parfois englobés et détruits, de sorte que Cú́not (1901) a pu considérer comme une loi générale l'existence chez ces parasites d'une chimiotaxie négative à l'égard des phagocytes. Cette opinion est partagée par BRASIL (1904); SiEdLECKI (1903), au contraire, après LÉGER (1897) et CAULLERY et MESNIL (1899), attribue cette indifférence des phagocytes envers le parasite à la mobilité de celui-ci. Les faits que nous venons de signaler vont à l'encontre de l'une et l'autre de ces manières de voir. $N$. vermicularis et $S t$. diadema sont assez agiles, et elles se meuvent même sous l'épaisse couche de phagocytes qui les recouvre; le mouvement du parasite n'a donc pas empêché ici l'englobement par les phagocytes ; mais il n'existe pas davantage d'action chimique répulsive envers ces organites, on ne peut en effet invoquer unétat pathologique qui annihilerait ce chimiotactisme, les individus phagocytés ne sont ni morts comme chez Diplocystis, ni malades comme chez Gonospora, puisque tous ou presque tous sont ainsi attaqués sans présenter cependant aucun phénomène de dégénérescence ni avant ni après l'attaque des phagocytes. L'hypothèse d'une sécrétion protectrice à laquelle se rallie BrasiL (1904) pourrait cependant être invoquée, il suffirait de supposer que cette sécrétion a pour effet, non plus d'éloigner les phagocytes, mais de défendre la Grégarine attaquée contre l'action des sucs digestifs de ces cellules.

Comment se nourrit la Grégarine enrobée ? Est-ce aux dépens des phagocytes qui l'entourent? Cette hypothèse si invraisemblable qu'elle paraisse pourrait cependant s'appuyer sur l'existence de parasites qui vivent aux dépens des lymphocytes, tels les Leucocytozoon des Oiseaux et des Mammifères, tels Orcheobius herpobdellae des testicules de Nephelis vulgaris, tel encore un parasite que nous avons rencontré au cours de ces 
recherches dans les vésicules séminales de Helodrilus caliginosus et que nous décrirons prochainement. Malheureusement, il ne nous a pas été possible de trouver la moindre trace d'altération dans les phagocytes qui entourent les parasites; ce n'est donc pas à leurs dépens que se nourrit la Grégarine. Il faut donc admettre que l'épais manteau phagocytaire n'est pas un obstacle pour les échanges nutritifs entre le parasite et le milieu ambiant et que les courants osmotiques traversent ces phagocytes comme une sorte de filtre.

\section{Iumbrici olidi.}

La dégénérescence granuleuse que nous avons rencontrée quelquefois chez les Grégarines précédentes paraît assez fréquente chez cette espèce. Les grains de paramylon disparaissent peu à peu, le cytoplasme se charge de granulations sphériques peu volumineuses qui se colorent en bleu pâle par le Mann, et il se vacuolise par places ; en même temps, la cuticule s'épaissit fortement et les poils tombent (fig. 80). Le noyau demeure assez longtemps normal, puis il subit la pycnose ; il y a ensuite disparition de la membrane nucléaire et la masse chromatique en pycnose se dissout dans le plasma environnant, de sorte qu'il ne reste d'autre trace du noyau qu'une plage cytoplasmique plus fortement colorée qui finit elle-même par disparaître. Le cytoplasme se condense en boules éparses dans une masse fluide (plasmarrhexis); parfois il y a formation d'une zone hyaline entre le cytoplasme granuleux en plasmarrhexis et la cuticule épaissie.

\section{St. coronata.}

Nous retrouvons encore chez St. coronata la dégénérescence granuleuse avec plasmarrhexis.

Ici, les phénomènes pathologiques débutent par une hypertrophie du noyau qui accompagne ou même précède la disparition des grains de paramylon (fig. 81,82 , etc.). Nous avons 
rencontré précédemment cette hypertrophie chez $M$. agilis, mais dans des cas très rares.

Les grains de réserve disparaissent, il apparaît en même temps dans le cytoplasme des gouttelettes que le Mann colore en rouge, puis ce cytoplasme se creuse de vacuoles en même temps qu'il devient granuleux, il se condense ensuite en boules assez fortement colorables séparées par de grands espaces clairs dans lesquels sont creusées les vacuoles. La ventouse a rapidement disparu. La cuticule se ratatine et disparaît; les sphères de cytoplasme se dispersent dans le fluide séminal où elles deviennent la proie des phagocytes.

Dans le noyau hypertrophié, le réseau de linine se gonfle de façon à rappeler vaguement l'aspect d'un spirème, mais d'un spirème fort peu chromatique; puis il s'en détache des fragments en forme de saucisse (fig. 84), la chromatine perd sa colorabilité, la membrane nucléaire se ratatine et disparaît et le karyosome se dissout dans le cytoplasme ambiant.

J'ai observé aussi parfois de curieux phénomènes de dégénérescence nucléaire. Chez un parasite où les grains de paramylon étaient en voie de disparition et où le cytoplasme était creusé déjà de grandes vacuoles et présentait de nombreux grains chromatoïdes, le noyau hypertrophié ne montrait plus ni karyosome, ni réseau de linine ; sa membrane elle-même n'était plus distincte, bien que ses contours restassent cependant bien tranchés. La zone cytoplasmique entourant le noyau était un peu plus colorée que le reste du cytoplasme. Le noyau devenu ellipsoïdal, allongé dans un sens perpendiculaire au grand axe du parasite, avait son suc nucléaire très fortement coloré par l'hématoxyline. A son intérieur la chromatine se présentait sous forme d'une série de bâtonnets renflés à leurs extrémités et disposés très régulièrement suivant une couronne équatoriale. La figure 86 montre cette disposition qui rappelle à s'y méprendre l'aspect d'une mitose au stade de métaphase, telle qu'on l'observe dans les kystes du même parasite pendant la multiplication des noyaux qui conduit à la 
formation des gamètes. Les éléments achromatiques étaient mal visibles, sans doute à cause de la coloration très foncée du suc nucléaire.

Dans un autre parasite (fig. 87), au contraire, ces éléments étaient très nets car ils étaient recouverts sur toute leur longueur par une fine poussière chromatique. Un tel fuseau n'est pas sans présenter quelque vague analogie avec celui que Brasil (1905, b. pl. IX, fig. 4) représente comme le $1^{\text {er }}$ type des mitoses chez les Monocystis de Lumbricus herculeus; mais il rappelle beaucoup plus cependant un fuseau d'Amibe ou de Cilié qu'un fuseau de Grégarine. S'il s'agit là d'une véritable mitose, ce serait donc une mitose tout à fait aberrante, tant par le moment où elle se produit que par l'aspect qu'elle présente.

Je n'ai pu vérifier si ces mitoses sont abortives ou si elles se poursuivent jusqu'à l'individualisation de deux noyaux fils. Quoi qu'il en soit, j'ai cru devoir signaler ces faits, car ils représentent un mode intéressant de réaction du parasite contre des conditions de vie défavorables, mode tout à fait curieux si l'on pense que, à l'état normal, les Monocystidées de même que toutes les autres Eugrégarines, ne montrent jamais de divisions pendant leurs stades végétatifs.

Tous ces phénomènes d'altération ont été observés dans les vésicules séminales de divers Pheretima hauayana à un moment où ces vésicules renfermaient de très nombreux éléocytes, éléments que l'on n'y rencontre jamais d'une façon normale. Il est fort possible qu'il y ait relation de cause à effet entre la présence de ces éléocytes et la dégénérescence des parasites ou plutôt que ces deux faits proviennent d'une même cause, un état pathologique des vésicules séminales

Il convient de mentionner aussi chez St. coronata quelques cas de dégénérescence hyaline du cytoplasme. Le noyau ne s'hypertrophie pas alors, il subit le karyorrhexis.

St. coronata est parfois entourée par les phagocytes de l'hôte ; elle est alors le siège des mêmes phénomènes que nous avons 
rencontrés dans les $M$. lumbrici phagocytées : hypertrophie notable, division en lobes profonds, vacuolisation et dégénérescence du cytoplasme.

Le noyau devient hyperchromatique, la membrane nucléaire disparaît ; le suc nucléaire fortement coloré ne laisse plus apercevoir dans son intérieur de corps figuré. Puis le noyau disparaît totalement; la Grégarine se fragmente en un grand nombre de lobules qui sont enveloppés et mangés par les phagocytes.

Les Grégarines accouplées peuvent être ainsi phagocytées au début de leur enkystement; elles s'hypertrophient et leur noyau devient fortement hyperchromatique ; mais il n'y a pas creusement de sillons superficiels séparant des lobes. Le plasma se remplit de grains chromatoïdes arrondis de taille variée. C'est le seul stade de dégénérescence que,j'aie observé, les stades ultérieurs m'ont échappé sans doute à cause de la rareté des cas rencontrés.

\section{Le Mêmei.}

M. Le Mêmei est assez souvent attaquée par les phagocytes de l'hôte. Les réactions que l'on constate alors chez ce parasite sont très comparables à celles que nous avons décrites chez d'autres espèces. La Grégarine s'arrondit, d'ovoïde elle devient sphérique; on constate dans son intérieur la disparition progressive des grains de réserve. Il apparaît dans le cytoplasme des gouttelettes que le Mann colore en rouge ; puis ces gouttelettes disparaissent, le cytoplasme subit la dégénérescence granuleuse et le plasmarrhexis.

Dans le noyau, le karyosome semble se dissoudre dans le suc nucléaire qui devient fortement colorable. La membrane nucléaire prend un contour irrégulier puis disparaît. Il ne reste d'autre trace du noyau qu'une plage fortement colorée qui ne tarde pas à disparaître à son tour.

A côté des divers cas de dégénérescence que nous venons de décrire et qui sont dus soit à l'attaque par les phagocytes 
de l'hôte, soit à une cause inconnue, il en est d'autres qui sont occasionnés par divers parasites envahissant les Grégarines et arrivant souvent à les détruire. Le chapitre suivant est consacré à la description de ces parasites et à l'étude de leur action sur la Grégarine qui les héberge.

\section{Parasites.}

On n'a décrit jusqu'ici que fort peu d'organismes parasites chez les Grégarines.

Caullery et Mesnil (1891) ont trouvé chez diverses Grégarines d'Annélides marines des parasites pour lesquels ils ont créé le genre Metchnikovella ; ils font rentrer dans ce genre les organismes signalés par CLAPARÈde (1861) chez une Grégarine de Phyllodoce et par LÉGer (1892) chez Sycia et Platycystis ; il convient d'y ajouter les formations signalées par LEIDY (1882) chez Monocystis mitis et considérées par cet observateur comme des spores de la Grégarine.

Caullery et Mesnil (1905) ont rencontré aussi chez certains Selenidium des inclusions parasitaires qu'ils rapprochent des Bertramia.

Dogiel (1906) a observé chez Cystobia chirodotae une Coccidie, Hyalosphaera gregarinicola dont il a pu suivre en grande partie le cycle évolutif.

Porter (1908) signale chez Merogregarina amaroucii de l'intestin de Amaroucium un organisme rappelant les Chytridiopsis de Schneider. Enfin Léger et DuboscQ (1909a) ont décrit récemment une Microsporidie du genre Nosema chez Frenzelina conformis Dies., de l'intestin de Pachygrapsus marmoratus F.

Tels sont les parasites connus jusqu'ici chez les Grégarines. Il convient peut-être de leur ajouter [MESNIL (1909)] les organismes observés par Awerinzew (1908) chez une Grégarine de l'intestin de Amphiporus et que l'auteur considère comme appartenant au cycle asexué de la Grégarine. 
Chez les Monocystidées de la cavité générale, nous n'avons jamais observé de parasites. En revanche ceux-ci existent souvent dans les Monocystidées de l'intestin ou dans celles des vésicules séminales.

Nous avons retrouvé dans $M$. mitis Leidy de l'intestin de Fridericia polycheta Bretscher, les organismes que Leidy

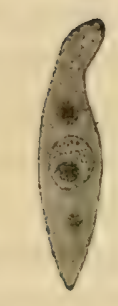

XCVT.

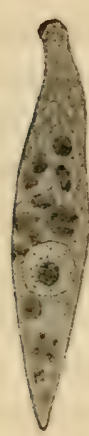

XCVII.

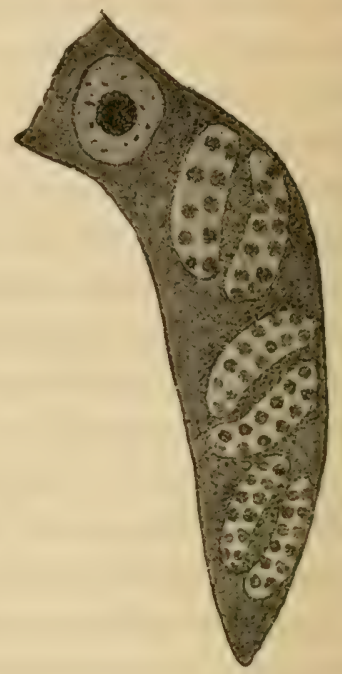

XCTIIT.

FIG. XCVI. M. mitis renfermant 2 individus de Metchnikovella dont l'un en vole de division nucléaire $\times$ 1.300. - FIG. XCVII. $\boldsymbol{M}$. mitis renfermant de nombreux noyaux de Metchnikovella $\times 1.300$. - FIG. XCVIII. Portion postérieure de $\boldsymbol{M}$. mitis avec kystes de Metchnikovella $\times 1.300$.

a décrits comme spores de la Grégarine. Ce sont là des Metchnikovella voisines de celles décrites par Caullery et Mesnil. Nous dirons peu de choses sur ces parasites, le matériel nous ayant manqué pour en faire une étude détaillée. Nous n'avons observé que des stades mono-ou pluri-nucléés représentés (fig. XCVI et fig. XCVII) et des kystes. Certaines figures font penser à une multiplication mitotique des noyaux. Les kystes sont naviculaires comme ceux de Metchnikovella spionis Caullery et Mesnil et renferment également de nombreux 
noyaux disposés en deux séries parallèles au grand axe du kyste (fig. xcviri).

Le noyau de l'hôte n'est pas altéré ; le cytoplasme est absorbé par le parasite, mais il ne paraît pas y avoir de réaction de défense. On aperçoit cependant autour des kystes un amoncellement de petits grains chromatoïdes qui sont probablement des grains d'excrétion rejetés par le parasite au moment de la sporulation.

Les Monocystidées des testicules ont aussi leurs parasites spéciaux; nous n'avons plus affaire ici à des Metchnikovella, mais vraisemblablement à des Bactéries. J'ai rencontré ces organismes chez $M$. lumbrici, $M$. agilis. $R$. pilosa, $M$. striata et St. coronata; chaque espèce a un Microbe qui lui est propre et que l'on ne rencontre jamais chez les autres. Ces Bactéries sont de petite taille, elles sont ovoïdes, en forme de bâtonnets courts ou de filaments longs et déliés, souvent elles renferment des condensations chromatiques en forme de noyaux.

Chez $M$. lumbrici, je n'ai rencontré des Bactéries que dans les individus phagocytés, en voie de dégénérescence. Ce sont des bâtonnets généralement rectilignes de $\mathrm{I}$ à $3 \mu$ de long, très minces, à cytoplasme fortement chromatique et présentant dans chacune de leurs extrémités légèrement renflées une masse plus fortement colorable que le reste de l'élément et qui pourrait être interprétée comme un noyau. Lorsque le bâtonnet est un peu allongé, il existe en outre une ou deux masses fortement chromatiques entre les amas terminaux (fig. 95).

Ces Microbes sont entassés dans les travées de spongioplasme qui séparent les grains de paramylon fortement gonflés et à cavité centrale volumineuse. Ce spongioplasme est très intensivement coloré par le Fer, on y rencontre de nombreuses granulations chromatophiles et des boules chromatoïdes analogues à celles que nous avons décrites dans un chapitre précédent; à un stade ultérieur, les boules chromatoïdes disparaissent et il ne reste qu'une masse granuleuse très fortement colorée dans laquelle il est difficile d'apercevoir les bâtonnets microbiens. 
Dans les régions du corps de la Grégarine où la dégénérescence est très avancée, les parasites semblent eux aussi en mauvais état, ils sont plus pâles et beaucoup moins nombreux que dans les régions où la dégénérescence est moins marquée.

Je n'ai pu suivre le sort de ces Bactéries après l'altération complète de la Grégarine, je ne sais donc rien sur leur mode de multiplication et de dissémination. Je n'ai jamais vu de Grégarine libre parasitée, la présence du Microbe paraît liée à celle des phagocytes, et cependant les Microbes semblent souffrir des attaques de ces phagocystes aussi bien que la Grégarine qui leur donne asile. Il y a là un phénomène curieux que je n'ai pas réussi à expliquer.

Chez les autres Monocystidées, les Bactéries ne se trouvent plus chez les individus phagocytés, elles attaquent les parasites d'apparence tout à fait normale.

Honocystis agilis peut être envahie de très bonne heure par un Microbe représenté (fig. 89). La Grégarine encore contenue dans le blastophore où elle s'est développée a déjà quelquefois son cytoplasme bourré de parasites. Ceux-ci se présentent comme des corps ovoïdes ou piriformes, accolés par deux et montrant une petite masse chromatique au voisinage de leur pointe, du côté opposé au point d'accolement. Certaines figures font penser à une division par scissiparité.

La colorabilité des parasites est très variable; à côté de formes présentant simplement un point coloré au voisinage de la pointe, on en aperçoit qui sont complètement et fortement imprégnés par le Fer. Les dimensions et la forme varient aussi considérablement. La forme la plus commune est celle que nous indiquons plus haut, mais, au lieu de s'accoler simplement deux à deux, les parasites peuvent se rassembler en plus grand nombre; en se plaçant bout à bout, ils constituent des filaments dont la longueur moyenne est de 6 à $8 \mu$, avec $\frac{1}{2} \mu$ d'épaisseur environ, mais parfois beaucoup plus grêles et dans lesquels on observe des amas chromatiques régulièrement espacés.

Je ne sais comment se produit l'infection pour les Grégarines 
encore renfermées dans les blastophores; mais j'ai pu observer les débuts de l'envahissement microbien chez des formes extracellulaires dont les extrémités étaient en train de se ratatiner et de s'atrophier pour donner naissance à un de ces parasites en sporocyste que nous avons décrits. Les Microbes encore peu nombreux sont logés à ce moment dans les extrémités tronquées et, de ces points, on voit de petites traînées microbiennes rectilignes se répandre dans le cytoplasme central que, sans doute, elles ne tardent pas à envahir complètement.

Dans les Grégairnes attaquées, on observe comme chez celles qui sont entourées par des phagocytes un gonflement considérable des grains de paramylon, puis leur disparition. Il y a formation de boules chromatoïdes analogues à celles que nous avons décrites chez les Grégarines normales et chez les Grégarines phagocytées et dont l'apparition semble coïncider avec la dissolution des grains de paramylon, puis ces boules s'évanouissent; dans les travées de spongioplasme, les fines granulations sidérophiles disparaissent peu à peu et le cytoplasme devient très pâle, clair et il est dépourvu de toute granulation colorable.

Le noyau n'est jamais attaqué, mais son altération suit celle du cytoplasme. Les granulations chromatiques qu'il renferme pâlissent et disparaissent; le karyosome perd à son tour toute colorabilité, la membrane nucléaire s'évanouit et il ne reste à la place du noyau qu'une plage irrégulière de plasma granuleux un peu plus coloré que le cytoplasme environnant par les colorants plasmatiques, surtout par le Vert lumière; puis il ne reste même plus de trace de cette plage colorée, le noyau a totalement disparu.

$M$. striata est attaquée aussi par un Microbe particulier dont l'aspect rappelle beaucoup celui des Microbes filamenteux de $M$. agilis ; mais les filaments sont plus déliés, recourbés et non rectilignes, en outre, ils s'enchevêtrent les uns dans les autres de façon à constituer de petits amas floconneux, sortes de zooglées qui emplissent le spongioplasme. Ici encore le 
noyau n'est pas attaqué par le parasite; sa dégénérescence n'est qu'une conséquence de celle du cytoplasme, elle se produit par un processus tout à fait semblable à celui que nous venons de décrire chez $M$. agilis; il en est de même du cytoplasme dont les grains de réserve disparaissent ainsi que les granulations colorables et qui devient ainsi un fluide homogène clair (fig. 90.)

Chez $R$. pilosa, nous retrouvons encore une autre Bactérie. Celle-ci ne prend jamais la forme filamenteuse ; elle est arrondie, ovoïde ou piriforme, rappelant celle qui attaque $M$.agitis, mais en général plus renflée, moins allongée. Elle présente également de grandes variations de taille.

La multiplication se fait par division binaire: le corps s'allonge en un bâtonnet aux deux extrémités duquel se trouvent dos masses chromatiques condensées, puis il se produit un étranglement médian suivi d'un étirement; les deux parasites formés peuvent rester quelque temps rattachés l'un à l'autre par un fin pédicule; parfois la division est inégale et l'on a un processus de bourgeonnement. La figure 94 montre divers aspects du Microbe au repos et en voie de division. Les processus de dégénérescence cytoplasmique et nucléaire ne diffèrent en rien de celles qui viennent d'être décrites pour les autres Monocystidées.

De même que les Grégarines des Lombriciens, St. coronata des Pheretima est quelquefois attaquée par des Microbes qui provoquent sa dégénérescence et sa mort.

L'envahissement peut se produire de très bonne heure alors que la Grégarine est encore à l'intérieur du blastophore nourricier (fig. 93), c'est ce que nous avons observé déjà chez M.agilis. Parfois St. coronata est encore très petite et l'on peut cependant voir déjà quelques Bactéries dans son cytoplasme. Au début, ce sont des bâtonnets courts montrant un point chromatique à chacune de leurs extrémités légèrement renflées; ces bâtonnets sont placés dans le voisinage du noyau, souvent même ils touchent par une des extrémités à la membrane nucléaire. 
Ils mesurent 4 à $5 \mu$ de long, se multiplient par un processus que je n'ai pu établir et envahissent tout le cytoplasme de l'hôte; ils s'allongent considérablement (fig. 91), se recourbent, s'enchevêtrent les uns dans les autres de façon à former dans l'intérieur de la Grégarine un paquet qui semble formé d'un filament continu. Le noyau est respecté, il n'y a jamais de parasite dans son intérieur.

Le cytoplasme des Stomatophora attaquées perd ses grains de réserves, il apparaît dans son intérieur des boules chromatoïdes qui s'évanouissent ensuite, puis le cytoplasme pâlit et il est absorbé bientôt par le parasite. Le noyau qui n'est pas attaqué directement dégénère à son tour; sa chromatine disparaît et son karyosome se décolore. Ce sont là les mêmes processus que nous avons déjà rencontrés chez les Monocystis du Lombric.

Pendant que se produisent ces altérations, les filaments bactériens qui ont absorbé tout le cytoplasme de leur hôte se rassemblent vers le centre, se pelotonnent autour du noyau, se tassent en formant une boule fortement colorable d'où s'échappent quelques filaments qui s'en vont jusqu'à la cuticule de l'hôte (fig. 92), celle-ci se plisse, se ratatine et vient s'appliquer plus ou moins étroitement sur la zooglée bactérienne. Ces sphères de Bactéries sont peut-être des formes de résistance qui se dissocieraient à un moment donné en leurs éléments bacillaires pour infester de nouveaux hôtes; mais je n'ai pas réussi à suivre leur destinée. Elles rappellent d'une façon frappante les pelotons myceliens de Rhizoctomes, représentés par BERNARD (1909) dans les cellules des Orchidées.

Parfois, ces zooglées se forment avant que tout le cytoplasme de la Grégarine hôte ne soit absorbé ; dans ce cas, les éléments qui les constituent sont moins allongés, moins étriqués, ce sont simplement de longs bâtonnets étroitement tassés les uns contre les autres et non des filaments entremêlés. Ils constituent ainsi une boule compacte séparée par une zone claire du cytoplasme en voie de dégénérescence de la Grégarine hôte. 
Toutes ces Bactéries sont peu communes et il est trop difficile de les isoler pour qu'on puisse en tenter la culture in vitro. Cette culture serait cependant indispensable pour une détermination systématique exacte, c'est pourquoi nous n'avons pas donné de noms à ces parasites, nous contentant de noter leur aspect et leur action sur l'organisme des Grégarines.

Il est intéressant de remarquer que lorsque l'on trouve dans un Oligochète une Grégarine infestée, la plupart des autres Grégarines de la même espèce qui cohabitent avec elle sont également attaquées. Ce fait a déjà été constaté pour les Metchnikovella par Caullery et Mesnil.

L'étude des processus de dégénérescence que l'on observe sur le parasite nous conduit à étudier maintenant ceux que cause ce parasite sur l'organisme de son hôte.

\section{CHAPITRE V}

\section{ACTION PATHOLOGIQUE SUR L'HOTE}

Nous avons vu en effet en décrivant les diverses espèces de Monocystidées qui font l'objet de ce travail, qu'elles produisent souvent des altérations profondes dans les organes de leur hôte. L'étude de ces altérations, outre la contribution qu'elle apporte à la pathologie cellulaire, va nous donner dans quelques cas des renseignements précieux sur l'évolution normale ellemême de la cellule.

Les phénomènes de dégénérescence provoqués par les Grégarines ont été décrits avec beaucoup de précision par un certain nombre d'observateurs; LAveran et Mesnil (1900), Siedlecki (1901), Brasil (1904) et surtout Léger et DuboscQ (1899 et 1902) nous ont fait connaître pour quelques Monocystidées et pour les Polycystidées intestinales les altérations de la cellule envahie : hypertrophie suivie d'atrophie, chromatolyse du noyau, etc., etc.

Les Monocystidées des Oligochètes n'ont pas été très bien 
étudiées à ce point de vue, les auteurs se contentent de dire par exemple pour les Monocystis qui parasitent les vésicules séminales que les spermatozoïdes portés par les blastophores attaqués se flétrissent et disparaissent, mais ils n'indiquent pas par quel processus se produit ce dépérissement.

Quelques auteurs, StEIN (1848) et LIEBERKhÜN (1855) entr'autres, considéraient le revêtement de cellules sexuelles qui recouvrent certaines de ces Grégarines comme des appendices piliformes de la cuticule de ces parasites. Sснмпрт (1854) en a reconnu la véritable nature, et montré comment la Grégarine s'en débarrasse d'un seul coup. Ruschhaupt (1885) remarque que le parasite emplit complètement le blastophore, en absorbant le contenu et même le cytoplasme des spermatozoïdes qui dépériraient et disparaîtraient peu à peu. DrzEıEEKI (1903) a noté également le dépérissement des spermatozoïdes et il croit, lui aussi, qu'ils disparaissent progressivement.

Les Monocystis ne sont pas les seules Grégarines qui attaquent les glandes séminales mâles des Oligochètes. Ersen (1895) a signalé dans ces organes d'autres Monocystidées, les Spermatobium; ces parasites pénètrent, non plus dans les blastophores, mais dans les spermatogonies. Ils n'altèrent même pas les noyaux des cellules hôtes et ne présentent jamais le revêtement de spermatozoïdes altérés qui est si fréquent chez diverses Monocystis des Lombrics.

Outre les Grégarines, il existe encore d'autres Protistes qui parasitent les cellules testiculaires. On y a signalé des Coccidies, des Myxosporidies, des Champignons, des Bactéries. L'action pathogène de ces divers organismes ayant été étudiée avec quelques détails, nous allons résumer brièvement les observations faites à ce sujet par les auteurs.

Dans les testicules de Polymnia nebulosa Mont., Sieduecki (1902-1907) a signalé Caryotropha Mesnili, Coccidie qui, de même que les Spermatobium envahit les spermatogonies et non les cytophores. Ce parasite amène l'hypertrophie de la cellule attaquée et de son noyau et détermine même des troubles 
dans les cellules voisines du même follicule; ces cellules continuent à se multiplier, mais elles sont arrêtées dans leur évolution et ne se métamorphosent pas en spermatozoïdes, elles se soudent même parfois en une masse syncytiale.

Cúnot (1902) nous a fait connaître Legerella testiculi, Coccidie parasite des testicules des Glomeris marginata Villers de Lorraine ; ici encore il y a hypertrophie de la cellule sexuelle qui est mangée complètement par le parasite, la membrane cellulaire subsistant seule.

Une autre Coccidie décrite par Schubert et Kunze (1906) vit également dans les testicules, c'est l'Orcheobius herpobdellae, parasite de Nephelis vulgaris. Ce Sporozoaire se loge dans une vacuole creusée dans le cytophore de l'hôte, l'action exercée sur ce cytophore et sur les cellules sexuelles n'a pas été décrite; mais, c'est encore une hypertrophie que le parasite détermine sur les lymphocytes dans lesquels se passe son cycle sexué. Kunze (1907) qui a repris l'étude du parasite déclare qu'il ne produit pas la castration parasitaire.

Gonobia colubri Mingazzini, Coccidie parasite du testicule de Zamenis viridiflavus est toujours, d'après MrNGazzINI (1892), extra-cellulaire et le développement des spermatozoïdes n'est pas affecté par sa présence.

Thelohan (1892) a observé dans les testicules de la Sardine une autre Coccidie, Coccidium sardinae, mais il n'a vu que des formes mûres extracellulaires ; l'action sur l'hôte n'a donc pas été étudiée.

Citons enfin la Coccidie karyophage de Drüner : Micrococcidium karyoliticum que l'on rencontre dans les noyaux des spermatocytes de la Salamandre, Drüner (1894) et du Rat, Bouin (1897) Elle détermine la vacuolisation du noyau et semble absorber de la chromatine.

Trois espèces de Microsporidies sont parasites des testicules:

Nosema sp. trouvée par Pfeiffer (1895) chez une larve de Potamanthus; N. thysanurae Pfeiffer des Podura aquatica, enfin $N$. bryozoïdes Korotneff de Alcyonella fungosa. 
Les altérations causées par les deux premiers parasites n'ont pas été étudiées par Pfeiffer; quant à $N$. bryozoïdes, KonotNEFF (1892) a décrit en détail l'effet qu'il produit sur les spermatoblastes du Bryozoaire qui l'héberge; il y aurait là une sorte de mycoplasmose et une altération spéciale des noyaux de la cellule-hôte; mais Stempell (1904) et LÉGER (1908) ont montré que KoRotneFF avait sans nul doute commis une erreur d'interprétation; il considère en effet comme noyaux des spermatoblastes de l'hôte les gros noyaux végétatifs du parasite que Stempell (1904) et nous-même (1905) avons fait connaître chez d'autres Microsporidies, il a done pris pour une celluie parasitée ce qui ne représente que le seul parasite.

Sporomyxa scauri Léger, Myxomycète parasite des Scaurus, étudié par LÉGER (1908) cause dans les testicules qu'il envahit des troubles considérables : les cellules sexuelles s'hypertrophient, puis la chromatine nucléaire se répand dans leur cytoplasme sous forme de boules et de grains irréguliers, finalement, elles diffluent. Au moment de la mitose, il y a désorganisation complète du spirème.

L'action des Microbes sur le testicule a été étudiée souvent dans les cas de tuberculose, d'orchite, de syphilis; mais la plupart des études faites à ce sujet avaient un but clinique; lorsque les lésions ont été décrites au point de vue anatomopathologique, les auteurs ont simplement signalé la structure microscopique des régions envahies, sans s'occuper spécialement des processus dégénératifs dont les cellules sexuelles sont le siège. Ces processus ont été au contraire étudiés avec soin dans les cas d'ectopie ou de sténose expérimentale du canal déférent. Bouin (1897), Félizet et Branca (1902) ont montré que dans ces cas les principaux phénomènes observés sont :

Dégénérescence hyaline ou désintégration du plasma.

Pycnose, karyorrhexis, caryolyse, vacuolisation du noyau.

Mitoses asymétriques, pluripolaires, désordonnées, arrêtées en cours d'évolution ou à développement abrégé.

Enfin amitose. 
Des dégénérescences de même ordre ont été signalées aussi par N. Marcozzi (1907) sous l'action des poisons de la tuberculose.

Nous trouverons également quelques phénomènes comparables dans les testicules des Oligochètes envahis par les Monocystis.

\section{Monocystidées intestinales.}

M. mitis n'exerce pas d'action appréciable sur l'intestin des Fridericia qui l'hébergent. Je n'ai même pas observé la chute des cils que Nusbaum signale chez Henlea sous l'action de Schaudinnella. Cependant, parfois les M. mitis se trouvent rassemblées en paquets volumineux qui oblitèrent presque complètement la lumière du tube intestinal et compriment fortement les cellules épithéliales, dans ce cas les cils de l'épithélium sont froissés, tassés, et ils peuvent même disparaître ; mais les cellules épithéliales ne paraissent nullement altérées. On y observe même des mitoses superficielles de régénération tout à fait semblables à celles que l'on peut voir dans les autres régions de l'intestin non attaquées.

\section{Monocystidées cœlomiques.}

Les Monocystidées coelomiques semblent n'amener aucune réaction sur les tissus de l'hôte envahi ; nous avons vu qu'elles sont, ou complètement libres dans la cavité générale, ou revêtues d'une couche de cellules conjonctives de l'hôte, aplaties et formant un manteau continu autour du parasite et le rattachant soit à la paroi du corps, soit à l'intestin, soit à la paroi des tubes néphridiens. On observe quelquefois des parasites dans l'intérieur même de ces tubes néphridiens, comme nous l'avons vu dans certains cas pour M. Michaelseni. Alors, les néphridies sont fortement distendues, boursouflées par la présence de la Grégarine, mais c'est là un fait rare et exceptionnel. 


\section{Monocystidées génitales.}

Parmi les espèces qui envahissent les vésicules séminales, nous avons vu que les unes restent constamment extracellulaires, tandis que les autres passent une partie de leur existence soit dans les cellules sexuelles, soit dans certaines cellules de la paroi des pavillons séminaux.

\section{Monocystidées eXtracellulatres.}

La plupart des Monocystidées extracellulaires n'exercent pas d'action sensible sur l'hôte; sans doute, elles détournent à leur profit une partie des substances nutritives destinées aux organes où elles se trouvent, sans doute aussi elles exercent une compression plus ou moins considérable sur les cellules qui les entourent; mais cette influence n'est pas directement observable, son étude est par suite très difficile sinon impossible.

Une espèce fait exception cependant, c'est $P l$. Cuenoti. L'action de ce parasite sur les cellules des pavillons séminaux rappelle beaucoup en effet celle qui est produite sur ces mêmes cellules par $N$. magna. Aussi, en étudiant l'action de cette dernière, étudierons-nous comparativement celle de $P l$. Cuenoti.

\section{Monocystidées INTRACELLULAIRES.}

Parmi les Monocystidées qui passent par un stade intracellulaire, nous nous occuperons d'abord de $N$. magna.

N. Magna. - Nous avons vu que l'on sait, depuis Bütschli (1881), que les $N$. magna sont fixées à la paroi des pavillons séminaux ciliés au moyen de leur extrémité antérieure qui est piquée, et souvent profondément enfoncée dans certaines cellules saillantes de cet épithélium, les " pokalförmige Zellen. "

Structure normale des pavillons ciliés. Avant d'étudier 
l'action du parasite sur sa cellule-hôte, il faut donc indiquer brièvement quelle est la structure normale de l'épithélium des pavillons.

Neuland (1886) a indiqué d'une façon très sommaire l'histologie de cet épithélium; depuis, Bugnion et Popoff (1905), Depdolla (1906) ont donné des figures représentant cet épithélium, pour montrer la façon dont les spermatozoïdes se fixent entre les cils de la surface; ces dessins sont trop schématiques, ils ne montrent pas les cellules saillantes qui se dressent sur cette surface et qui sont facilement visibles soit que l'on examine un fragment dilacéré des pavillons, aplati entre lame et lamelle, soit que l'on étudie une coupe transversale de leur paroi. Les figures Lxi et 97 représentant un lambeau d'épithélium dilacéré et un fragment de coupe transversale montrent très bien l'aspect de ces cellules.

Les cellules épithéliales sont appliquées contre l'épithélium péritonéal formé de cellules très aplaties, à noyau petit et allongé. Elles sont peu élevées, mais assez larges et renferment un cytoplasme granuleux parfois dense, mais le plus souvent grossièrement alvéolaire surtout vers la base de la cellule. Le noyau volumineux, sphérique ou ellipsoïdal contient un gros nucléole central ou sub-central et un réseau lininien à mailles en général peu serrées, sur lequel sont dispersés les grains chromatiques. Le bord libre de la cellule présente un plateau cuticulaire strié qui porte la bordure de cils vibratils entre lesquels s'engagent les têtes de spermatozoïdes mûrs emmagasinés dans les pavillons. De distance en distance, on voit faire saillie à la surface de cet épithélium des cellules coniques ou ellipsoïdales, rattachées à l'épithélium par une large base, ou portées par un pédoncule court et étroit ce qui leur donne l'aspect de massues (fig. 97). Le cytoplasme de ces cellules, très dense vers la base, au voisinage du point d'attache devient vacuolaire dans la région distale; celle-ci s'allonge parfois considérablement en s'amincissant. Le bord libre m'a toujours semblé dépourvu de plateau cuticulaire, cependant 
il est hérissé d'aspérités coniques qui sont peut-être l'origine de cils épaissis à leur base. Le noyau est plus petit que celui des cellules épithéliales et plus fortement colorable. Ces cellules saillantes, assez abondantes au voisinage du bord libre des pavillons, deviennent de moins en moins nombreuses à mesure que l'on se rapproche du fond de l'entonnoir et même finissent par disparaître. Je n'ai pu établir d'une façon certaine quelle est leur origine et quel est leur rôle. La figure 100 montre une de ces cellules dans le cytoplasme de laquelle sont englobés de nombreux spermatozoïdes enchevêtrés; à côté d'elle, dans l'épaisseur de l'épithélium, on voit une autre cellule bourrée de balles granuleuses jaunâtres qui sont probablement des produits d'excrétion ou des résidus de digestion. Ces cellules à grains jaunes ou brunâtres, de même que les cellules en saillie remplie de spermatozoïdes plus ou moins altérés ne sont pas rares dans les coupes. Il conviendrait peut-être de considérer les cellules en saillie comme des organes phagocytaires comparables dans une certaine mesure aux organes cilio-phagocytaires signalés par Cuénot (1902 b), Schneider (1899), Kowalewsixy (1896) dans les néphrides de divers Oligochètes ou Hirudinées.

Cellules cupuliformes. On aperçoit en outre d'ici de là sur l'épithélium du pavillon les cellules spéciales dans lesquelles sont implantées les $N$. magna et que nous avons décrites précédemment. De même que les cellules phagocytaires, elles font saillie à la surface de l'épithélium auquel elles sont rattachées par un pédoncule court et étroit; elles ont la forme d'une coupe régulière, d'où le nom de "pokalförmige Zelle " que leur a donné Bütschli (1881); parfois basses et évasées, parfois étroites et allongées, ces cellules sont toujours creusées en leur centre d'une cavité cratériforme dans laquelle se trouve logée l'extrémité antérieure du parasite (fig. 98, 101, 102, 103). Le pourtour de cet orifice porte de longs cils à base élargie ; le reste de la surface de la cellule est également orné de cils, mais ceux-ci sont moins longs et plus minces, rappelant davantage l'aspect de ceux qui bordent les cellules épithéliales nor- 
males. Dans la région de contact, les cils ont disparu, la surface de la cellule est creusée de sillons dans lesquels s'enfoncent les côtes du parasite.

Le cytoplasme des cellules cupuliformes est finement granuleux comme celui des cellules épithéliales; mais il présente des différenciations très particulières, caractéristiques de la " cellule en bocal ». Dans l'axe de cette cellule, on observe un cordon épais, fortement colorable par les colorants chromatiques et qui, partant du fond de la coupe traverse le pédoncule et arrive jusqu'à la base de la cellule où il se divise en de nombreux rameaux divergents qui rayonnent dans l'épithélium et sont visibles à de très grandes distances (fig. LXIII, 101, 102, 103).

Ce cordon est formé de fibrilles ondulées, très étroitement serrées les unes contre les autres et qui appartiennent partie à la cellule elle-même, partie à la Grégarine ; ces dernières étant moins longues et moins nombreuses, ainsi que nous l'avons dit déjà dans un chapitre précédent. Tout autour de ce tronc axial, d'autres fibrilles moins développées divergent vers la périphérie de la cellule où elles se mettent en rapport avec la portion basilaire des cils (fig. 101).

Le noyau est logé dans les parois latérales de la coupe, quelquefois au voisinage du tronc fibrillaire central. Il renferme un gros nucléole peu colorable et un réseau lâche, à filaments très fins sur lesquels on observe de rares grains chromatiques très petits.

Le cytoplasme des cellules cupuliformes présente parfois une structure qui rappelle celle que nous avons signalée dans la région distale des cellules phagocytaires saillantes. Près du fond de la cavité, au voisinage du noyau, on peut voir en effet quelquefois une région creusée de larges alvéoles ; mais ce n'est pas là une structure normale; d'autres cellules montrent des alvéoles d'une grandeur considérable et qui ont envahi la plus grande partie du corps cellulaire (fig. 102, 103), il s'agit sans nul doute d'une altération dont le premier résultat est de faci- 
liter la libération du parasite et qui conduit à l'atrophie et à la disparition de la cellule cupuliforme. La Grégarine n'étant plus solidement enchâssée dans la cellule-hôte quitte en effet son support et de la cellule bocal il ne reste bientôt d'autres traces que ses nombreuses fibrilles basilaires rayonnantes intra-épithéliales.

La présence d'un cytoplasme alvéolaire dans les cellules cupuliformes, la situation de ces cellules à la surface de l'épithélium pourraient porter à les considérer comme proches parentes des cellules phagocytaires; mais, nous venons de voir que l'existence de ce cytoplasme vacuolaire est simplement un signe de dégénérescence, en outre la cellule en bocal se rapproche nettement des cellules épithéliales ordinaires par la présence d'un revêtement ciliaire, par la structure ordinaire du cytoplasme et par celle du noyau, de sorte que, pour nous, i! n'est pas douteux qu'elle ne provienne de la modification de l'une de ces cellules : modification produite par une hypertrophie considérable et par la différenciation de fibrilles dans son intérieur.

Il eût été intéressant de suivre les diverses phases de ces transformations; malheureusement, nous n'avons que bien peu de documents sur le développement de la cellule cupuliforme. En décrivant le développement de $M$. magna nous avons signalé quelques stades de cette modification; mais les cellules les moins transformées que nous ayons pu observer faisaient déjà fortement saillie hors de l'épithélium et présentaient également des fibrilles assez développées (fig. 98). Nous n'avons pas vu les premiers effets de la pénétration du parasite. L'hypertrophie qu'il amène dans la cellule-hôte est considérable et elle paraît se produire avec une grande rapidité. Le parasite d'abord complètement intracellulaire fait ensuite hernie hors de la cellule-hôte, sa région antérieure restant enfoncée dans la cavité qu'il a creusée dans cette cellule. A mesure qu'il grandit la cellule-hôte s'hypertrophie davantage et elle finit par atteindre jusqu'à 80 à $100 \mu$ de diamètre vers l'ouverture de la cavité, 
avec une hauteur de 45 à $50 \mu$, l'épaisseur des parois de la coupe étant de 15 à $20 \mu$. Nous sommes loin des dimensions primitives de la cellule épithéliale qui ne dépassent guère $20 \mu$ sur $25 \mu$.

Nous venons de dire qu'il nous avait été impossible d'observer le début des altérations amenées par le parasite dans la cellule-hôte, néanmoins, nous avons rencontré, à la vérité très rarement des cellules épithéliales qui présentaient un aspect intermédiaire entre la cellule épithéliale normale et la jeune cellule cupuliforme déjà fortement saillante. La figure 106 représente une de ces formations; elle est beaucoup moins surélevée que la cellule représentée (fig. 98) et qui contient une jeune $N$. magna, en outre elle est rattachée à l'épithélium par toute la largeur de sa base et non par un pédicule étroit. Elle montre à sa surface des cils normaux fins et serrés s'échappant d'un plateau un peu plus développé que celui des cellules normales. J'ai retrouvé un semblable plateau légèrement modifié dans une autre cellule hypertrophiée, il présentait ici de larges stries semblables à celles que montre la cellule cupuliforme et les cils qui s'en échappaient moins nombreux que dans le cas précédent étaient beaucoup plus épais surtout à la base. Le noyau de ces cellules, peu modifié, laisse cependant apercevoir une légère hypertrophie et son nucléole a perdu en partie sa colorabilité. Le cytoplasme pariétal renferme quelques différenciations fibrillaires qui sont en rapport avec la base des cils.

J'ai cherché en vain des parasites dans ces cellules. Cependant, on remarque en leur centre, au voisinage du noyau une sorte de cavité dont la paroi est creusée de rainures (fig. 106) et dont l'intérieur est fortement colorée en vert par le Vert lumière et d'une consistance très homogène. $Y$ aurait-il eu là un très jeune parasite? Je ne sais, en tous cas il n'en reste pas de trace. Sur les bords latéraux et au-dessous de cette cavité il existe une région assez étendue où le cytoplasme au lieu d'être dense et finement granuleux comme dans le reste du 
corps cellulaire est au contraire creusé de nombreuses alvéoles petites et régulières. Cette région cytoplasmique alvéolaire est en rapport direct avec des fibrilles radiculaires divergentes semblables à celles que nous avons signalées dans les cellules cupuliformes, mais beaucoup moins développées.

Racovitza (1896) a montré que, chez les Polychètes, les cellules épithéliales présentent à leur extrémité profonde une série de prolongements fibrillaires qui s'accolent entr'eux, se tassent de façon à constituer la basale. Les fibrilles radiculaires que nous venons de décrire doivent sans doute être homologuées aux fibres basales des cellules épithéliales des Polychètes. Ces fibrilles ne sont pas visibles dans les cellules normales car elles sont très fines et si étroitement tassées qu'elles constituent une lame compacte, cependant, si, au lieu de considérer les cellules du pavillon cilié, on étudie celles qui forment la paroi des canaux déférents, on remarque très bien à leur base un enchevêtrement de fins filaments qui sont un prolongement des cellules. Ces cellules rappellent du reste dans une certaine mesure celles de l'épithélium des pavillons, elles présentent de même un plateau cuticulaire portant une bordure de longs cils et elles reposent sur les cellules plates de l'épithélium péritonéal. Leur noyau a la même structure : gros nucléole et réseau de linine assez serré sur lequel sont dispersés les grains chromatiques (fig. 104). Mais, elles sont moins larges et plus hautes que les cellules du pavillon et leur cytoplasme a un aspect tout différent; les filaments basaux que nous avons vu s'intriquer pour constituer la basale semblent se prolonger dans la cellule elle-même et la traverser dans toute sa longueur pour aller s'insérer à la base des cils. Cet aspect fibrillaire a été déjà décrit dans les cellules épidermiques d'autres Oligochètes; il rappelle tout à fait celui que nous avons rencontré dans les cellules cupuliformes.

La traction considérable exercée par le parasite sur la cellule-hôte aurait ainsi amené dans le cytoplasme de celle-ci la différenciation de fibrilles analogues à celles qui existent 
normalement dans les cellules des canaux; ces fibrilles se seraient ensuite hypertrophiées par suite du travail considérable qu'elles ont à fournir et seraient devenues très résistantes; en s'accolant les unes aux autres, elles constituent le tronc fibrillaire que nous avons décrit dans l'axe du pédoncule de la cellule en bocal. Ces fibrilles sont naturellement restées en connexion avec les filaments basilaires qui se sont, eux aussi hypertrophiés et ont pris un développement énorme. Mais au lieu de rester en place et de former une couche lamellaire sous l'épithélium, ils ont été déplacés par la traction et se sont pour ainsi dire enchâssés dans les cellules épithéliales sus-jacentes, constituant les racines intra-épithéliales si développées de la cellule cupuliforme.

PL. Cuénoti. - Il est très curieux de constater que les effets pathologiques produits par $N$. magna qui passe manifestement par un stade intracellulaire se retrouvent chez une espèce qui est sans doute extra-cellulaire pendant tout son développement. $P l$. Cuenoti en effet est fixée à l'épithélium du pavillon séminal des Helodrilus par des cellules très comparables aux "pokalförmigen Zellen». Mais ici la cupule dans laquelle est enfermée la tête du parasite n'est plus constituée par une cellule unique, mais par un assemblage de cellules assez étroitement serrées les unes contre les autres, et dont chacune présente les caractères de la cellule cupuliforme (fig. 99):

Hypertrophie considérable du corps cellulaire qui fait saillie hors de l'épithélium; hypertrophie des cils et hypertrophie du noyau dans lequel on observe un volumineux nucléole peu colorable et un réseau lininien lâche avec rares grains chromatiques à sa surface. Modification du plateau cuticulaire dan la région du contact; disparition des cils en ce point et forma tion de rainures dans lesquelles s'engrènent les côtes ectoplas miques du parasite. Différenciation de fibrilles très développées dans le sein du cytoplasme, fibrilles qui vont du plateau ou de la base des cils aux filaments basilaires de la cellule par lesquels elles se continuent; hypertrophie de ces filaments 
basilaires qui sont enchâssés dans les cellules épithéliales voisines et s'étendent à de grandes distances.

On compte jusqu'à dix do ces cellules, parfois davantage, qui se serrent les unes contre les autres pour constituer ensemble une cupule où sont logées les extrémités antérieures des deux parasites accolés. Ici, la fixation n'est plus assurée que par l'engrènement des côtes du parasite dans les sillons du plateau cuticulaire des cellules-hôtes; le pourtour de la cupule en effet n'enserre pas étroitement le corps de la Grégarine comme nous l'avons vu pour $N$. magna, l'adhérence est cependant considérable et suffisante pour maintenir le parasite et produire les mêmes altérations cytologiques de la cellule-hôte que nous avons constatées à propos de $N$. magna.

Les débuts de ces altérations m'ont complètement échappé. Je n'ai jamais observé chez Helodrilus longus et $H$. caliginosus de cellules hypertrophiées dans l'épithélium du pavillon, en dehors de celles qui sont soumises à l'action des parasites; la structure de cet épithélium est du reste analogue à celle qui a été décrite chez Lumbricus herculeus.

Monocystidées intrablastophoriennes. - Toutes les Grégarines dont il nous restẹ à étudier l'action sont parasites des blastophores de l'hôte; mais elles agissent non seulement sur ce blastophore lui-même dont elles absorbent la substance, mais encore sur les cellules sexuelles qu'il supporte. L'évolution de celles-ci ne parvient pas à s'achever et elles dépérissent avant de pouvoir se transformer en spermatozoïdes. Il y a ainsi une véritable castration parasitaire directe, mais c'est là une castration toujours partielle et incomplète, car, à côté des blastophores envahis, il en subsiste beaucoup qui demeurent intacts et qui supportent des spermatozoïdes normaux en nombre suffisant pour assurer la fécondité de l'hôte.

Cette castration retentit-elle sur l'organisme de l'hôte et produit-elle des troubles analogues à ceux que les recherches de Giard nous ont fait connaître sur un grand nombre d'animaux et de végétaux? Nous avons cherché à nous procurer des 
Oligochètes indemnes de parasites pour comparer leur organisation à celle des individus qui hébergent des Monocystis; nous n'avons malheureusement pas eu la bonne fortune de rencontrer un seul exemplaire de Lombric ou de Pheretima qui ne soit infesté. Tous les animaux étudiés, même très jeunes, et encore dépourvus de clitellum, mais à capsules séminales développées, nous ont montré un nombre plus ou moins considérable de parasites. En ce qui concerne les Lombries, comme nous l'avons dit précédemment, e'est surtout chez ces jeunes animaux que nous avons trouvé le plus abondamment les premiers stades du développement des Monocystis. Il nous a done été impossible de vérifier les observations de Bugnion et Popoff (1905). D'après ces auteurs, la structure normale des capsules séminales du Lombric serait considérablement modifiée par la présence des Grégarines et de leurs kystes; mais ils ne signalent pas d'autre retentissement sur l'organisme de l'hôte.

Nous savons déjà que l'action de nos parasites se fait sentir à la fois sur le blastophore et sur les cellules sexuelles que supporte ce blastophore, nous allons étudier successivement les phénomènes dégénératifs qui se produisent ainsi au cours de l'évolution du parasite.

\section{Action sur le Blastophore.}

En étudiant le développement des Grégarines, nous avons vu qu'elles peuvent envahir le blastophore à divers stades de son évolution; souvent même le parasite attaque la spermatosphère avant la formation du blastophore. Chez $M$. agilis où la pénétration est toujours précoce, la jeune Grégarine se loge dans le petit amas de cytoplasme résiduel qui est au centre $\mathrm{du}$ follicule spermatique, au point de jonction des pédicules des diverses cellules.

Le cytoplasme abandonné par les cellules séminales en voie 
de développement et qui, normalement sert à la formation du blastophore, s'accumule d'une façon régulière autour du parasite qui l'absorbe peu à peu.

En ce qui concerne les autres espèces, l'infection d'un follicule peut également avoir lieu à un stade très précoce de son évolution; nous avons observé par exemple des jeunes St. coronata en train de pénétrer dans le pédicule de spermatocytes de premier ordre (fig. 138) avant l'individualisation du blastophore. Mais l'envahissement peut aussi se produire à un stade ultérieur et même lorsque le blastophore, complètement dénudé a laissé s'échapper toutes les cellules sexuelles qui le recouvraient (fig. 43). Je n'ai observé ce dernier cas qu'une seule fois; il doit être très rare; cependant, il est intéressant à noter, car il semble y avoir là un véritable fourvoiement du parasite. Le blastophore qui a achevé son évolution ne tarde pas en effet à devenir la proie des phagocytes et le parasite auquel il donne asile partage son sort et est détruit en même temps que lui.

La plupart des $R$. pilosa que j'ai observées étaient dans des blastophores recouverts de spermatocytes II ou de spermatides, cependant, j'ai noté aussi quelques cas d'invasion plus précoce.

Quel que soit l'instant où le parasite se loge dans le blastophore, son action sur celui-ci reste la même. Il se creuse une cavité dans le cytoplasme et se nourrit aux dépens de ce cytoplasme qu'il absorbe peu à peu. Dans certains frottis colorés, cette cavité paraît beaucoup plus volumineuse que le parasite lui-même, mais ceci est dû sans doute à l'action des réactifs fixateurs, je n'ai jamais observé le fait in vivo, on ne le trouve pas non plus dans les préparations fixées à l'aide du Flemming fort qui conserve admirablement les structures.

Le parasite grossit aux dépens de la substance du blastophore, il finit par l'absorber complètement et par emplir tout à fait ce cytophore; celui-ci est alors déformé et fortement distendu. Alors que, chez L. herculeus, les blastophores normaux ne dépassent pas $45 \mu$ de diamètre ou $60 \mu$ sur $30 \mu$, on observe des blastophores parasités ayant jusqu'à $150 \mu$ sur $50 \mu$. En 
outre, le cytophore qui est normalement sphérique ou elliptique épouse les contours du parasite et prend la forme d'un fuseau allongé ou d'une toupie. Chez Octolasium lacteum Örley, et chez les Pheretima, le gonflement n'est pas aussi considérable, les blastophores normaux les plus volumineux atteignent chez Octolasium $40 \mu$ de diamètre au maximum, et chez Pheretima, $24 \mu$ sur $15 \mu$; quand ils sont parasités, ils ne dépassent guère $83 \mu$ sur $70 \mu$ chez Octolasium et $45 \mu$ sur $38 \mu$ chez Pheretima. On n'observe pas non plus un notable changement dans la forme du blastophore, le parasite restant lui-même sphérique ou elliptique; chez Octolasium cependant le parasite, $M$. turbo, présentant une légère pointe postérieure, le cytophore prend ce même aspect en toupie.

On pourrait être tenté de comparer cette distension à l'hypertrophie constatée par les auteurs dans les cellules qui hébergent des Grégarines ou des Coccidies. Cette comparaison serait inexacte, le blastophore envahi ne semble pas du tout réagir contre le parasite, il reste complètement passif, et ce fait n'a rien de surprenant si nous pensons que le blastophore, dépourvu de tout noyau ainsi que nous l'avons montré récemment (1909) n'est pas une cellule, mais une simple masse de cytoplasme inerte, réserve de substance nutritive pour les cellules séminales ou plutôt reliquat de cytoplasme inutile abandonné par ces cellules au cours de leur développement. Il n'y a donc pas ici une hypertrophie véritable mais une dilatation purement mécanique. Cette dilatation n'existe pas pour $R$. pilosa qui fait de bonne heure hernie hors du blastophore et quitte celui-ci sans le distendre.

Lorsque la Grégarine emplit complètement le blastophore distendu, on observe que le parasite est entouré par une mince cuticule assez fortement colorée par le Fer et qui est étroitement appliquée sur son épicyte (fig. 115, 121, 122, 159, 162, etc.); c'est à cette membrane que sont fixés les restes plus ou moins altérés des cellules sexuelles. Une telle membrane n'existe pas dans les blastophores normaux où elle opposerait une résis- 
tance au détachement des spermatozoïdes. Sous l'action de certains réactifs fixateurs, cette membrane se détache de l'ectoplasme du parasite auquel elle est le plus souvent étroitement accolée, et elle devient ainsi facilement visible. La Grégarine doit briser cette membrane pour devenir libre dans le fluide qui emplit les vésicules séminales. On observe souvent dans les frottis de telles enveloppes abandonnées par les parasites et supportant leur revêtement de cellules sexuelles altérées. Elles finissent par être englobées par les phagocytes de l'hôte de même que les blastophores dont l'évolution est terminée.

D'où provient cette membrane qui ne se trouve pas dans les blastophores normaux? Ce pourrait être une réaction de défense de la cellule-hôte contre le parasite; mais le blastophoren'étant pas une véritable cellule, il est peu probable qu'il réagisse de la sorte. On ne constate pas du reste autour du parasitece tassement des couches cytoplasmiques voisines que l'on rencontre presque toujours dans les kystes de réaction. Il est facile de suivre la différenciation de cette membrane. Au début de l'envahissement, elle n'existe pas. Certains réactifs fixateurs déterminent, ainsi que nous l'avons dit plus haut, la formation d'une cavité plus ou moins grande autour du jeune parasite; les parois de cette cavité ne montrent tout d'abord aucune différenciation, puis elles paraissent formées par une série de très petites granulations présentant certaines réactions de la chromatine et qui notamment se colorent avec une certaine intensité par le Fer; ces granulations deviennent de plus en plus serrées et finalement constituent une surface continue. Ceci a lieu de très bonne heure pour $M$. agilis et $R$. pilosa; mais en ce qui concerne St. coronata, on ne l'observe guère que lorsque le parasite emplit complètement le blastophore, de sorte que c'est la surface externe de ce blastophore qui semble s'être ainsi couverte d'une membrane différenciée.

Cette membrane est due probablement à l'accumulation de produits d'excrétion provenant du parasite. C'est une 
membrane assez résistante qui peut gêner dans une certaine mesure l'expansion de ce parasite. On voit en effet, certains individus de $M$.agilis obligés de reployer sur eux-mêmes une de leurs extrémités avant de pouvoir s'échapper de l'enveloppe. Celle-ci doit néanmoins être très perméable puisqu'elle n'empêche pas les échanges nutritifs entre l'hôte et le parasite. Il est très curieux de constater qu'elle n'empêche pas non plus l'attraction sexuelle, beaucoup de St. coronata en effet s'accouplent et s'enkystent sans sortir de leur enveloppe blastophorienne ainsi que nous l'avons vu en décrivant cette espèce.

ACTiON SUR LES CELLULES SEXUELLES SUPPoRtÉES PAR LE BLAS'TOPHORE.

L'action du parasite sur les cellules sexuelles est notoirement plus sensible que sur le blastophore, elle semble varier beaucoup avec le moment où se produit l'infection et aussi avec l'espèce de parasite; ainsi, les altérations produites par $M$. agilis paraissent très différentes de celles qu'occasionne $R$. pilosa, celles-ci par contre rappellent étonnamment les déformations causées par St. coronata. Malgré ces différences, on peut démêler dans ces processus pathologiques des phénomènes généraux communs à tous les parasites.

Le premier phénomène que l'on constate dans toute spermatosphère envahie par une Monocystidée quelle qu'elle soit, c'est une attraction par le parasite, du noyau des cellules sexuelles. Ces noyaux qui occupent la région distale dans les cellules séminales normales : spermatogonies spermatocytes ou même spermatides au début, se rapprochent tous de la région proximale tandis que le cytoplasme s'amasse dans la partie distale. Cette attraction des noyaux par le parasite a été déjà signalée par LÉGER et DuBoscQ (1899-1904) pour Gregarina Davini Léger, et pour Stylorhynchus longicollis F. St. et S. oblongatus Hainn; par BrasiL (1908) pour Doliocystis pellucida Köll.; par SredLecki (1907) pour Caryotropha Mesnili 
Siedlecki. C'est là sans doute une réaction défensive d'ordre général, car on a noté de tels déplacements du noyau dans d'autres cas, ainsi E. TANGL (1884) a montré que dans des éléments blessés, les noyaux des cellules qui avoisinent la plaie se rapprochent de la lésion

Au terme ultime de l'altération du noyau de la cellule sexuelle, cette action attractive du parasite sur la chromatine est encore très manifeste, cette substance en effet s'accumule dans la région proximale et le noyau prend l'aspect d'une masse dense conique, plus ou moins allongée dont la base élargie est tournée vers le blastophore parasité.

L'attraction parasitaire peut aussi amener le passage d'une partie de la chromatine de la cellule sexuelle dans le blastophore; ce phénomène que nous avons observé (1909) normalement dans la spermatogenèse des Lumbriculus, beaucoup plus rare chez Allobophora et Lumbricus, ne se présente plus chez Pheretima que comme processus pathologique dû à l'action du parasite (fig. 147).

Les autres modes de dégénérescence de la cellule sexuelle sont d'apparence extrêmement variée, mais ils peuvent tous, en dernière analyse être considérés comme une sorte d'accélération des processus normaux de la spermatogenèse. Les variations sont dues à ce que, au moment où elle est envahie par le parasite, la spermatosphère peut se trouver à divers stades de l'évỏlution.

Nous allons étudier avec quelques détails ces variations pour chaque parasite. Nous indiquerons d'abord les processus de dégénérescence dans les cellules au repos; ensuite nous signalerons les troubles apportés dans le phénomène de la mitose.

Monocystis AgiLIs. - Nous savons que cette Grégarine pénètre dans les groupes folliculaires au moment où les cellules sexuelles sont aux premiers stades de leur développement, et cela toujours avant l'individualisation du blastophore. Le follicule peut donc être envahi soit lorsque les cellules séminales sont à l'état de spermatogonies, soit lorsqu'elles sont à l'état 
de spermatocytes I. En rapport avec ces deux époques d'entrée du parasite, nous observons deux types d'altération.

$1^{0}$ Les cellules étaient au stade de spermatogonies au moment de la pénétration du parasite. - Sous l'action de la Grégarine, elles prennent la forme d'une lanière plus ou moins régulière, renflée à sa base tournée vers le blastophore, pointue et quelquefois laciniée à l'autre extrémité. Le noyau arrondi est logé dans la base renflée.

$2^{\circ}$ Les cellules étaient au stade de spermatocytes I quand le follicule a été envahi. - Le terme ultime de l'altération de ces cellules est représenté par un bloc de chromatine plus ou moins compact, souvent creusé de vacuoles, en forme de cône allongé, dont la base est tournée vers le blastophore ; parfois cette masse de chromatine est recouverte d'une très mince couche de cytoplasme flétri.

I. Pénétration précoce. - Le parasite peut envahir la spermatosphère dès qu'elle est tombée du testicule; dans ce cas, les cellules sexuelles se resserrent autour de lui, se disposant en une couche régulièrement circulaire ; la phase de dissociation est supprimée, mais les mitoses se produisent avec leur nombre normal bien qu'elles soient le plus souvent atypiques, comme nous le verrons plus loin. Les cellules séminales entourant le parasite seront ainsi deux ou trois fois plus nombreuses que celles qui environnent un blastophore normal et elles seront aussi plus petites que les cellules normales.

Si la pénétration n'a lieu que plus tard, le nombre de ces cellules est plus restreint au début, mais le parasite paraît exercer sur les cellules sexuelles une sorte d'excitation à la multiplication de sorte que le nombre des mitoses augmente et que les cellules sexuelles recouvrant le blastophore parasité peuvent devenir excessivement nombreuses.

Dès que le parasite est installé au centre d'un follicule, nous pouvons observer la migration déjà signalée plus haut, des noyaux vers ce centre ; ils se disposent en une couche régulière autour du parasite. Comme conséquence de ce fait, la 
masse cytoplasmique des spermatogonies qui était accumulée au point de naissance des pédicules (Cf. Hesse 1909) se trouve rejetée dans la région distale et l'idiozome la suit dans cette migration ; (comparer fig. 107-110) ; ici encore, comme dans la spermatogenèse normale, l'idiozome se trouve toujours là où est accumulée la plus grande quantité de cytoplasme. Cet idiozome a la forme d'une sphère dont la surface est souvent fortement colorée par le Fer, de sorte qu'il se présente alors en coupe optique comme un anneau. Cet anneau est tangent à une baguette courte, étroitement appliquée contre la membrane nucléaire, qui se colore aussi avec intensité par le Fer et qui représente vraisemblablement l'appareil centrosomien.

Le cytoplasme distal, d'abord très dense, se creuse bientôt do vacuoles nombreuses. L'appareil idiozo-centrosomien ne reste pas longtemps appliqué sur la membrane nucléaire, il émigre bientôt vers la surface. La baguette centrosomienne s'accroît vers l'extérieur, poussant devant elle une faible couche du cytoplasme distal ; elle s'allonge en un long appendice filiforme le long duquel s'écoule une quantité de plus en plus grande de cytoplasme; il se constitue ainsi une sorte de lanière cytoplasmique parfois assez épaisse, mais parfois aussi filiforme dont l'axe est parcouru par un filament coloré par le Fer (fig. $124,125,126)$.

Dans son mouvement de migration, l'idiozo-centrosome ne change pas d'orientation, de sorte que la direction de la baguette centrosomienne reste tangentielle au lieu de devenir radiale comme c'est le cas normal (fig. 123); comme conséquence de ce fait, le filament cytoplasmique s'échappe suivant une direction tangentielle; cette disposition subsiste quelque temps, mais elle finit par disparaître. La baguette centrosomienne se renfle vers sa base tangente à l'idiozome, et elle forme une masse conique bien visible à la base del'étirement cytoplasmique. Etant donnée l'origine de cet étirement, il n'est pas tout à fait homologable à la queue du spermatozoïde, nous devons plutôt le considérer comme formé par la coalescence de la queue 
et de l'aussenkörper. Comme il se produit d'une façon plus précoce que ces formations, alors que les cellules sexuelles possèdent encore une masse cytoplasmique considérable puisqu'elles n'en ont pas abandonné encore pour la formation du blastophore, au lieu de donner un filament grêle, il constitue une formation cylindro-conique assez épaisse.

Idiozome et centrosome d'abord bien visibles à une faible distance du noyau s'en éloignent notablement par suite de l'allongement de la cellule; puis ils deviennent moins distincts, semblant s'émietter dans le cytoplasme caudal et subissant avec lui la dégénérescence vacuolaire ou granuleuse.

Dans les cellules normales, on sait que le centrosome reste en relation avec la membrane nucléaire. Cette relation existet-elle ici ? Le plus souvent il est impossible de l'observer; centrosome et noyau sont assez éloignés l'un de l'autre et il ne semble pas y avoir entr'eux d'appareil de liaison différencié ; mais ce fait est peut-être dû à l'état de dégénérescence du cytoplasme. Au début, lorsque ce cytoplasme est encore dense et finement granuleux au lieu d'être vacuolaire, on peut voir parfois entre la membrane nucléaire et le centrosome une traînée de fines granulations colorées par le Fer et qui représenteraient cet appareil d'union tout à fait fugace et transitoire (fig. 125, 126). Parfois, il semble que le centrosome se dédouble en un grain distal et un grain proximal et on aperçoit celui-ci sur la membrane nucléaire (fig. 125). On voit que le cytoplasme se comporte ici à peu près comme celui de la spermatide en voie d'allongement. Les phénomènes qui se déroulent dans le noyau, au contraire s'éloignent assez notablement de ceux que l'on observe dans les noyaux des spermatides normales. Nous avons représenté (fig. 113, 123, $127,128,129,130)$ les principaux stades de la spermatogenèse normale auxquels on pourra comparer les stades pathologiques que nous allons décrire.

La chromatine qui, dans les noyaux normaux de spermatogonies (fig. 113), spermatocytes I (fig. 127) et spermato- 
cytes II (fig. 128) est distribuée d'une façon à peu près régulière sur tout le réseau, s'accumule en certains points. Cette condensation va en s'accentuant et toute la chromatine finit par se rassembler en ces points de concentration, le réseau de linine disparaît et l'on n'observe plus dans l'intérieur du noyau qu'une série de grains chromatiques variables en grosseur et en nombre et qui s'accolent à la membrane nucléaire sur laquelle ils forment une couche plus ou moins continue. Cette concentration s'éloigne notablement de celle que l'on observe dans les spermatides normales, on s'en rend compte facilement en comparant à la figure 123 (spermatides normales) les figures 124, 125, 126 (stades pathologiques).

C'est là un mode de dégénérescence qui rappelle le karyorrhexis des auteurs et que l'on pourrait comparer à ce que BouıN (1897) a observé dans les spermatocytes de jeunes rats en préspermatogenèse. Mais il faut noter que, dans les cellules en karyorrhexis, on voit finalement la membrane nucléaire disparaître et les boules chromatiques se disperser dans le cytoplasme ambiant, tandis que dans nos cellules séminales en dégénérescence, nous n'avons jamais observé ces faits. La membrane nucléaire reste intacte et la dégénérescence ne va pas plus loin. Le noyau diminue simplement de volume à mesure que s'étire le cytoplasme; il ne se condense pas non plus en un bloc compact, et ne s'étire pas comme dans la spermatogenèse normale.

La Grégarine rompant à ce moment la paroi du blastophore quitte celui-ci auquel demeurent attachés les restes des cellules sexuelles. L'ensemble ne tarde pas à devenir la proie des phagocytes qui errent en tout temps dans les vésicules séminales.

Il faut sans doute considérer comme appartenant au cycle de dégénérescence que nous venons de décrire les formations représentées (fig. 115), le noyau a en effet le même aspect que celui de la figure 126, mais la lanière cytoplasmique est moins régulière et l'on n'aperçoit pas dans son intérieur l'appareil idiozo-centrosomien. 
II. Pénétration tardive. - Si le parasite envahit la spermatosphère lorsque celle-ci est formée de spermatocytes I, les phénomènes dégénératifs précédents se produisent d'abord: étirement du cytoplasme en lanière et vacuolisation de ce cytoplasme, rassemblement de la chromatine en grains plus ou moins volumineux qui s'accolent à la membrane nucléaire. Puis l'étirement du cytoplasme se poursuit, le filament diminue d'épaisseur et devient presque filiforme, la vacuolisation s'accentue et la plus grande partie du filament devient granuleuse et se flétrit, il n'en subsiste guère que la portion basilaire dans laquelle est logé le noyau. Dans ce cas, l'idiozo-centrosome ne paraît pas jouer un rôle aussi important que dans le cas précédent, du moins, je ne l'ai aperçu qu'au début du phénomène d'étirement, et dans quelques cellules seulement (fig. 107, 108 et 110), plus tard (fig. 114, 116, 117, etc.), on n'en retrouve pas trace. Le noyau est arrondi, avec ses grains de chromatine accolés à la membrane, il présente encore parfois des traces d'un réseau, mais d'un réseau à filaments hyperchromatiques (fig. 114). Il s'allonge en même temps que le cytoplasme et devient elliptique; l'extrémité distale se rétrécissant plus rapidement, il prend ensuite une forme ovoïde, puis conique (fig. 116-117). Les grains de chromatine isolés se trouvent rapprochés par suite de ce processus d'étirement, ils viennent en contact et se soudent. On a ainsi une masse chromatique plus ou moins compacte en forme d'alène. L'aspect de cette masse est très variable suivant le moment où s'est produit l'allongement. Si celui-ci a lieu avant la disparition complète du réseau, on a les figures 117 ou 131 : la masse chromatique est assez épaisse et creusée de vacuoles, plus large à la base, plus mince vers la pointe, parfois irrégulièrement moniliforme; dans le cas où la chromatine se trouvait en grains volumineux peu nombreux, on a l'aspect indiqué par les figures 118 et 122 , ou, avec une condensation plus accentuée la figure 120. L'étirement se poursuivant, le volume de la chromatine diminue parce qu'elle se tasse davantage et les cellules sexuelles altérées pren- 
nent un aspect voisin de celui des spermatozoïdes normaux (fig. 121), parfois, des vacuoles creusées dans la masse chromatique leur donnent l'aspect des poils de $R$. pilosa (fig. 119).

On comprend qu'il peut y avoir entre ces diverses altérations une nombreuse série de formes intermédiaires; c'est ce qui a lieu en réalité et la variété de formes des cellules flétries est considérable.

Ici encore, nous trouvons une certaine ressemblance entre ces processus pathologiques et le karyorrhexis ou la pycnose des auteurs, suivant les cas. Mais il est peut-être plus simple et plus rationnel de voir là un véritable processus d'accélération de développement mais un processus morbide et qui ne donne naissance qu'à des produits mal formés ou avortés et non fertiles, voués du reste à une destruction rapide, puisqu'ils sont attaqués et englobés par les phagocytes de l'hôte lorsque la Grégarine les abandonne. On trouve en effet dans la cellule malade comme dans la cellule saine la même tendance à l'étirement qui donne une production filiforme, et, dans certains cas, le même rôle du centrosome et de l'idiozome dans cet étirement; on constate dans le noyau pathologique comme dans le noyau normal une condensation de la chromatine en un mince bâtonnet cylindrique ou conique plus ou moins allongé.

Les grandes lignes de l'évolution sont done les mêmes, mais dans les cas pathologiques, cette évolution est plus rapide et elle est arrêtée en cours de route. L'étirement du cytoplasme est moins considérable, et il subit la dégénérescence vacuolaire, puis granuleuse, se flétrit et même disparaît à peu près totalement; la condensation de la chromatine est moins complète, et elle se produit d'une façon tout à fait irrégulière. En outre, l'action attractive du parasite se faisant sentir, cette masse chromatique est toujours faiblement renflée à la base, par suite conique et non cylindrique. On ne constate pas non plus le changement de nature de la chromatine que nous avons signalé dans les noyaux normaux, Hesse (1909). Ces masses chromatiques résiduelles se colorent en effet toujours en bleu par le 
Mann et non en rouge ; si donc le noyau altéré présente une tendance à prendre l'aspect morphologique d'un noyau de spermatozoïde normal sa nature chimique reste différente, il dégénère toujours avant de mûrir.

Ces divers phénomènes nous offrent un nouvel exemple de l'indépendance relative du noyau et du cytoplasme, indépendance si souvent relatée par les observateurs, Hennegux (1894), W. Roux (1885), Israèl et Pappenhem (1895), Bouin (1897), pour ne citer que ceux-là. Nous trouvons un noyau normal de spermatogonie ou de spermatocyte dans un cytoplasme dont l'aspect morphologique est celui de la spermatide c'est-à-dire déjà étiré en lanière, ou même qui est déjà en train de subir la dégénérescence vacuolaire; puis nous voyons ce noyau subsister et s'allonger alors même que le cytoplasme a presque complètement disparu.

Nous avons vu précédemment que les cellules attaquées conservent le pouvoir de se multiplier par mitose et même que ce pouvoir semblait exalté par la présence du parasite puisque certains blastophores parasités supportent cinq ou six fois plus de cellules sexuelles que les blastophores indemnes. SiedLECKI (1907) a remarqué que les cellules sexuelles de Polymnia nebulosa parasitées par Caryotropha Mesnili conservent de même ce pouvoir de multiplication; mais leur évolution est arrêtée, elles ne modifient pas leur forme et restent à l'état de spermatogonies, de plus les mitoses sont normales, tandis que $M$. agilis amène des troubles assez profonds dans les phénomènes de la karyokinèse.

Si l'envahissement a eu lieu peu de temps avant la division des noyaux, on n'observe aucun trouble dans les manifestations de l'activité nucléaire; nous avons ainsi pu observer des stades de synapsis tout à fait normaux; rien non plus de changé dans les diverses phases de la mitose.

Lorsque le parasite a envahi le follicule dès sa sortie du testicule, on observe cependant déjà quelques différences: Dans le synapsis, la chromatine paraît plus tassée, on distingue diffici- 
lement les diverses anses du peloton chromatique qui sont fortement serrées les unes contre les autres. Dans le spirème, le ruban chromatique est plus épais, plus court, moins régulier, des chromosomes s'écartent de l'ensemble et paraissent même s'en séparer; puis il y a une condensation considérable, tout se tasse en un bloc irrégulier, les chromosomes aberrants formant un autre bloc isolé (fig. xcrx). Les mitoses sont alors asymétriques (fig. c). Je n'ai pu en suivre toutes les phases, mais j'ai observé le dédoublement de certains blocs chromatiques alors que d'autres restaient indivis; sans doute les chromosomes isolés se mettent au fuseau d'une façon irrégulière et ne se partagent pas également entre les cellules filles.

L'irrégularité du spirème et l'asymétrie des mitoses s'accentuent à mesure que l'évolution s'avance. Et, lorsque la cellule sexuelle est fortement étirée en lanière, on peut constater qu'au stade spirème, il y a formation dans une cellule de trois ou quatre blocs chromatiques très inégaux qui se distribuent d'une façon fort irrégulière entre les cellules filles. La division cytoplasmique est elle-même inégale et l'on a sur un même blastophore parasité, des cellules trois ou quatre fois plus volumineuses que leurs voisines, avec un noyau beaucoup plus riche en chromatine.

Rhynchocystis pilosa. - Au contraire de ce qui a lieu pour $M$. agilis, $R$. pilosa paraît envahir de préférence les blastophores bien individualisés et recouverts de cellules arrivées au stade de spermatocytes II ou de spermatides. Je l'ai observée très rarement dans les blastophores entourés de spermatocytes et n'ai pu suivre les altérations qu'elle occasionne alors, en dehors d'une condensation rapide de la chromatine.

En ce qui concerne les spermatides, le parasite est presque toujours situé excentriquement; la cavité qu'il se creuse dans le blastophore est tangente en un point (le point de pénétration probablement) à la surface externe du blastophore. Cette cavité est très vite tapissée par une membrane de même nature, je pense, que celle qui se forme pour $M$. agilis, mais plus épaisse et 
plus colorable (fig. 132). L'attraction des noyaux n'est pas sensible ici, du moins au début ; cependant elle était encore manifeste dans les cas d'envahissement précoce signalés ci-dessus. Par suite de ce fait, les manifestations spéciales que nous avons suivies dans le cytoplasme des cellules parasitées par $M$. agilis, ne se produisent pas ici; le cytoplasme suit son évolution naturelle. Quelquefois cependant, on peut constater une certaine accumulation de cytoplasme vacuolaire dans la région distale de la cellule; accumulation parfois considérable au point de modifier la forme de cette cellule; celle-c1 reste en effet piriforme, mais sa grande base est tournée vers la périphérie et non vers le centre de la spermatosphère comme c'est le cas normal. La forme de la spermatide rappelle alors celle des spermatocytes de premier ordre attaqués par $M$. agitis.

Lorsque l'élongation de la spermatide a lieu, cette masse cytoplasmique distale s'allonge et devient cylindrique; elle ressemble alors beaucoup à celle que nous avons observée à propos de $M$. agilis ; mais elle ne peut pas lui être comparée complètement. On aperçoit en effet à son extrémité un filament mince, filiforme (future queue du spermatozoïde), à côté duquel on peut constater le plus souvent la présence d'un aussenkörper très mince, mais assez allongé et nettement visible. Pour bien établir les homologies qui existent entre cette lanière et celles des cellules altérées par $M$. agilis, il conviendrait de distinguer dans ces dernières deux parties, l'une distale, de l'extrémité libre à la masse idiozo-centrosomienne; l'autre proximale, de cette masse au noyau; c'est cette dernière partie seulement qui correspond à la lanière tout entière des cellules attaquées par $R$. pilosa.

Au moment où le parasite fait hernie hors du blastophore, tout en restant fixé à celui-ci par son épimérite, il se produit un appel considérable de substance vers cet épimérite, les cellules qui entourent le bord de l'orifice de sortie du parasite s'appliquent étroitement contre la région antérieure de la Grégarine; dans celles qui sont plus éloignées, on constate une migration 
du noyau et du cytoplasme dans l'intérieur du pédicule qui se renfle; le cytoplasme vient grossir la masse du blastophore, le noyau restant logé dans le pédicule ; bientôt tout le blastophore est absorbé, il reste une masse assez dense formée de noyaux que le parasite abandonne bientôt et qui ont subi des altérations plus ou moins profondes.

Lorsque la Grégarine vient de pénétrer dans un blastophore, il se produit très rapidement une condensation de la chromatine dans l'intérieur des noyaux; cette substance se tasse au centre de l'aire nucléaire en un bloc arrondi ou ovalaire, d'abord rempli de petites vacuoles, puis excessivement compact et qui est entouré par une zone de suc nucléaire clair incolore (fig. 133 , $134,135)$. L'aspect du noyau est alors celui d'un noyau de spermatide normale au stade de condensation chromatique; il est seulement un peu plus petit à cause de la compacité plus considérable de la chromatine.

On observe parfois à ce moment une zone de cytoplasme différencié appliquée contre le noyau dans sa région proximale : quelle est la signification de cette zone? Idiozome ou nebenkern. Je n'ai pu établir son origine, mais il est fort probable qu'il ne s'agit pas là de l'idiozome car on constate en même temps la présence d'un aussenkörper dans lequel se trouve logé cet idiozome; si celui-ci est en réalité mal visible cela tient vraisemblablement à la vacuolisation du cytoplasme.

Le noyau s'allonge tout en gardant sa compacité, il devient conique, puis naviculaire et même quelquefois cylindrique; j'en ai observés qui atteignent jusqu'à 10 à $12 \mu$ de long avec $1 \mu$ d'épaisseur (fig. 135).

Les noyaux appliqués contre l'épimérite du parasite ou contre son extrémité antérieure sont beaucoup plus altérés; au lieu de s'allonger régulièrement, ils prennent des formes variables : blocs massifs irréguliers, massues, haltères à masses inégales, comme on peut le voir sur la figure 134 .

La cellule sexuelle pousse ici son évolution beaucoup plus loin que celles qui sont altérées par M. agilis, sans doute à cause 
de la pénétration tardive de $R$. pilosa; non seulement sa forme se rapproche davantage de celle du spermatozoïde mûr, mais encore, on peut observer ici le changement de nature de la chromatine qui se colore en violet ou en rouge par le Mann, avant même de s'être allongée complètement en fuseau ou en cylindre. Il y aurait donc ici encore accélération de développement, accélération qui retentirait jusque sur la nature chimique de la chromatine

Nous devons noter une disposition particulière des cellules sexuelles autour de $R$. pilosa, disposition que nous retrouverons fréquemment à propos de St. coronata. Dans le voisinage du point où la cavité intrablastophorienne creusée par le parasite est tangente à la surface du blastophore, on constate que les cellules sexuelles, ne sont pas implantées normalement à cette surface comme cela a lieu habituellement, elles sont toutes courbées dans le même sens et tendent à s'appliquer contre le blastophore. Nous chercherons plus loin, à propos de $S t$. coronata, l'explication de cette particularité

Les phénomènes que nous venons de décrire se retrouvent dans les spermatosphères parasitées par $R$. porrecta et $M$. striata, ou tout au moins des phénomènes très voisins, nous savons en effet que les stades intracellulaires de ces deux espèces sont tout à fait semblables à ces mêmes stades chez $R$. pilosa.

M. тURBo. - Nous retrouvons ici les altérations déjà décrites pour $M$. agilis, ou du moins des altérations de même ordre : augmentation du nombre des mitoses et, par suite, du nombre des cellules sexuelles, avec diminution du volume de celles-ci, allongement $d u$ cytoplasme en un filament mince mais néanmoins beaucoup plus épais que la queue des spermatozoïdes, vacuolisation, puis flétrissement de ce cytoplasme ; rassemblement de la chromatine en une masse assez condensée mais toujours vacuolaire, plus ou moins allongée.

Le parasite pouvant pénétrer ici dans un blastophore plus âgé que dans le cas de $M$. agilis, l'évolution de la cellule sexuelle peut se poursuivre plus longtemps et nous en avons observé 
qui étaient arrivées au stade de spermatide à noyau en bâtonnet cylindrique; mais l'aspect le plus général se rapproche de celui qui est représenté (fig. 118.)

St. CORonata. - Nous trouverons pour cette espèce une variété beaucoup plus considérable d'altérations; ceci est dû à ce que la pénétration du parasite dans la spermatosphère peut avoir lieu à n'importe quel stade du développement de celle-ci, dès l'instant où elle se détache du testicule pour tomber dans les vésicules séminales jusqu'à la fin de son évolution et même lorsque, cette évolution terminée, le blastophore dénudé va devenir la proie des phagocytes.

Nous distinguerons comme pour M. agilis, deux cas :

I. Pénétration précoce : le follicule est envahi dès sa sortie du testicule.

II. Pénétration tardive : le follicule est envahi à un stade ultérieur de son développement.

I. Pénétration précoce. Les phénomènes déjà décrits pour $M$. agilis se rencontrent également ici où l'on observe des phénomènes de métaplasie analogues à ceux que SiEdLECKI a signalés (1907) pour Caryotropha Mesnili.

Lorsqu'un parasite pénètre au centre d'un follicule qui vient de tomber des testicules, dans le cordon cytoplasmique qui sert d'union aux diverses cellules sœurs, ce cordon ne s'étire pas comme d'habitude, les cellules se massent régulièrement en cercle autour du parasite, la phase de dissociation est supprimée, mais les mitoses normales ayant lieu, la spermatosphère finit par être formée d'un nombre considérable de cellules sexuelles, disposées le plus souvent sur plusieurs cercles concentriques (fig. 144); nous avons pu compter plus de deux cents de ces cellules autour d'une volumineuse Grégarine (fig. 145).

Les noyaux émigrent aussitôt vers le centre du groupe de façon à entourer le parasite, le cytoplasme s'accumule dans la région distale puis il s'étire rapidement, de telle sorte que la spermatogonie devient piriforme (fig. 144). L'étirement se 
poursuivra jusqu'à ce que la cellule soit transformée en un long appendice cylindro-conique plus ou moins épais, mais très rarment filiforme (fig. 144, 145, 146). C'est absolument ce que nous avons constaté pour $M$. agilis, sauf en ce qui concerne l'idiozo-centrosome que nous n'avons pas observé ici. L'homologie se poursuit pour les phénomènes nucléaires. Les noyaux gardent longtemps l'aspect typique des noyaux de spermatogonies normales, avec un ou deux nucléoles très apparents; puis la chromatine se rassemble en quelques grains d'abord dispersés sur le réseau; le réseau disparaît ensuite et les grains chromatiques s'accolent à la membrane nucléaire qui devient assez fortement chromatique. Toutes les cellules et leurs noyaux gardent sensiblement le même volume, ce qui porte à croire que les mitoses restent normales, cette supposition est encore confirmée par le fait que les phénomènes dégénératifs ne se produisent que tardivement dans le noyau. Malheureusement, ceci est une simple hypothèse, je n'ai pu observer aucune division.

Le termine ultime de l'altération est représenté par la toison des Grégarines velues (fig. 145) que nous avons décrites en étudiant St. coronata.

II. Pénétration tardive. - La pénétration peut dans ce cas avoir lieu soit avant, soit après l'individualisation du blastophore.

a. Le parasite envahit les groupes de spermatocytes de premier mire. - Nous savons, (Hesse 1909) qu'à ce stade, le blastophore n'existe pas ou est à peine ébauché. Le parasite pénètre dans le pédicule d'un spermatocyte, au voisinage du centre de la spermatosphère (fig. 138), nous n'en avons jamais observé qui s'enfonce dans la région distale d'une cellule sexuelle.

Presque aussitôt après l'envahissement se produisent les phénomènes habituels d'attraction des noyaux; le cytoplasme au lieu de se rassembler dans la région proximale des cellules se réfugie dans la partie distale. Le blastophore restera par 
suite un peu moins volumineux qu'un blastophore normal. Le cytoplasme distal des cellules s'étire en une lanière assez épaisse et pointue à son extrémité qui peut atteindre jusqu'à $15 \mu$ de long sur 2 à $3 \mu$ d'épaisseur. Cette action sur le cytoplasme rappelle exactement celle du cas précédent; mais l'action exercée sur le noyau est toute différente. Dans chaque noyau, la chromatine se rassemble en une masse compacte, sphérique (fig. 157), présentant 2 à $3 \mu$ de diamètre et qui devient de plus en plus compacte (fig. 150) en même temps qu'elle s'étire en un court bâtonnet (fig. 149). Ces masses chromatiques sont colorées en bleu foncé ou en violet par le Mann et gardent cette coloration jusqu'au moment où elles sont phagocytées.

b. Le parasite envahit les groupes de spermatocytes de deuxième ordre ou les groupes de spermatides à divers stades de leurs métamorphoses.

Ici, deux facteurs interviennent pour modifier l'action du parasite : d'abord l'époque de la pénétration, puis le volume du blastophore.

L'influence de la Grégarine sur les cellules sexuelles ne se fait pas sentir en effet dès le moment de sa pénétration dans le blastophore, mais seulement lorsque la presque totalité ou tout au moins une grande partie de ce blastophore a été absorbée. A partir de cet instant, le cytoplasme et le noyau des cellules commencent à réagir et l'altération est rapide.

L'action combinée de ces deux facteurs peut produire une série de transformations très variées ; mais il n'est pas toujours facile de discerner la part qui revient à chacun d'eux.

Le dernier terme de l'altération de la spermatide paraît être la disparition à peu près complète du cytoplasme et la condensation de la chromatine en une masse compacte d'aspect assez variable : aciculaire, naviculaire, cylindrique, ovoïde ou sphérique. C'est sous ces divers aspects que l'on aperçoit les spermatides lorsqu'elles recouvrent des Grégarines parvenues au terme de leur croissance et ayant distendu fortement la membrane qui s'est formée autour du parasite à la périphérie du 
blastophore; ce sont aussi ces sortes d'altérations que l'on observe formant des paquets chiffonnés dans les vésicules séminales lorsque le parasite a rompu son enveloppe et s'en est dépouillé. On retrouve encore ces masses chromatiques caractéristiques dans les phagocytes qui ne tardent pas à les englober lorsque la Grégarine les a abandonnées.

Comme nous l'avons dit plus haut, l'action du parasite sur les spermatides ne devient sensible que lorsque la plus grande partie du blastophore a disparu. A ce moment, la Grégarine exerce sur le noyau des cellules sexuelles son action attractive habituelle; il en résulte que, d'une façon passive pour ainsi dire, le cytoplasme s'accumule dans la région distale de ces cellules. Il s'étire en une lanière plus ou moins épaisse suivant le volume qu'il présentait encore dans la cellule au moment où l'influence du parasite a commencé à se faire sentir. La figure 152 montre le début de cet étirement. Dans les figures 160 et 161 il est un peu plus accentué. Nous voyons aussi dans la figure CI la disposition des centrosomes et de l'idiozome.

L'idiozome légèrement hypertrophié est placé dans l'axe de la cellule, à côté de lui, la baguette centrosomienne un peu oblique par rapport à cet axe se prolonge dans l'intérieur de la cellule et ensuite à l'extérieur en un filament grêle, début de la queue.

Les deux grains centrosomiens, placés à une faible distance l'un de l'autre sont très distincts, ainsi que le filament qui les réunit. Ils sont légèrement éloignés du sommet du noyau ; mais, dans certains frottis colorés soit par le Mann, soit par le Fer-Vert lumière, le grain proximal paraît placé au sommet d'un cône cytoplasmique différencié qui a sa base sur le noyau (fig. 153). Il n'est jamais situé à une grande distance de ce noyau, comme nous l'avons observé chez Lumbricus ; mais le grain distal peut être très éloigné du grain proximal, sans doute par suite de l'étirement exagéré de la lanière cytoplasmique.

Nous avons vu que la baguette centrosomienne pouvait se trouver placée obliquement par rapport à l'axe de la celulle, 
comme chez Lumbricus ; dans ce cas, l'étirement du cytoplasme se fait dans le prolongement de cet axe, alors, filament caudal et lanière cytoplasmique constituent deux productions indépendantes (fig. 166, et fig. CII). On peut voir l'idiozome, très hypertrophié parfois, à la base de la lanière, ce qui semblerait

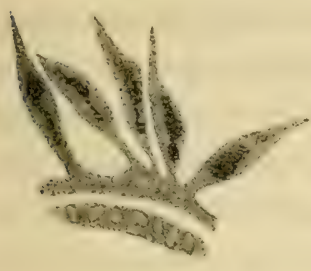

XCTY.

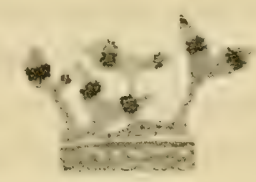

C.

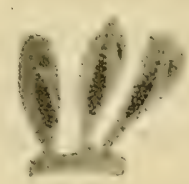

$C L$

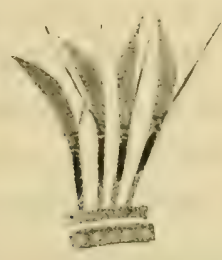

CII

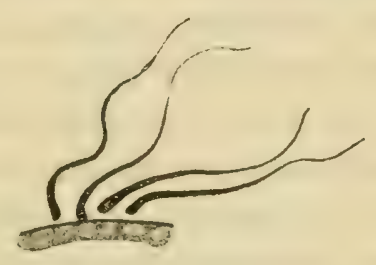

CIII

FIG. XCIX. $L$. herculeus, $M$. agilis dans un blastophore. Le spiresme est très tassé et irrégulier dans les cellules sexuelles $\times 1.300$. - FIG. C. $L$. herculeus. $M$. agilis dans un blastophore. Mitoses asymétriques des cellules sexuelles $\times 1.300 .-$ FIG. CI. Blastophore de Pheretima envahi par St. coronata $\times 1300$. Etirement du cytoplasme; appareil centrosomien dédoublé; idiozome latéral.- FIG. CII. St. coronota dans un blastophore de Pheretima $\times 1.300$. Le cytoplasme des spermatides s'étire dans une direction différente de celle du filament caudal. - Fig. CIII. St. coronota dans un blastophore de Pheretima. Spermatozoïdes altérés $\times 1.300$.

indiquer que cette lanière doit être comparée à un "aussenkörper ").

Nous avons malheureusement observé ce phénomène trop rarement pour pouvoir nous prononcer avec certitude sur ce point; le plus souvent en effet, le filament caudal est placé dans l'axe de la lanière cytoplasmique étirée. Cette lanière occupe généralement une situation radiale, mais il arrive aussi que les cellules, au lieu d'être disposées ainsi normalement 
à la surface du blastophore offrent une disposition que nous avons décrite déjà à propos de $R$. pilosa; elles se courbent toutes dans le même sens et même peuvent s'appliquer contre le blastophore. L'origine de cette courbure peut être trouvée dans la direction oblique primitive de la baguette centrosomienne qui donne naissance au filament, la lanière cytoplasmique suivant ensuite ce filament. Il est fort probable aussi que cette courbure soit accentuée dans la suite par un mouvement de rotation que le parasite imprimerait au blastophore qui lui sert d'asile. Cette courbure en effet s'observe seulement dans les blastophores que la Grégarine emplit presque complètement et qui sont peu volumineux; le parasite peut ainsi facilement les mettre en mouvement.

Lorsque la Grégarine a absorbé toute la substance du blastophore, elle peut rompre la membrane qui le recouvre, se dégager de cette enveloppe et vivre libre dans le fluide séminal. Très souvent cette rupture n'a pas lieu de suite, le parasite reste enfermé dans son étui et continue à grossir semblant sucer le cytoplasme des cellules sexuelles dont le volume diminue considérablement et qui disparaît peu à peu. On peut suivre l'étirement du cytoplasme et ensuite sa disparition graduelle dans les figures $153,154,162,163,164$ qui représentent une série de transformations de la cellule sexuelle. La comparaison des figures $152,161,158$ et 159 qui représentent une autre série d'altérations laisse voir également cette diminution de volume du cytoplasme des cellules sexuelles autour de blastophores de plus en plus distendus par les parasites qu'ils contiennent. L'existence d'une véritable succion semble corroborée par le mouvement des noyaux altérés qui viennent tous se réunir en face de la ventouse de la Grégarine (fig. 142).

Voyons maintenant comment se modifient ces noyaux.

L'action de la Grégarine se manifeste moins rapidement sur la chromatine des cellules sexuelles que sur leur cytoplasme ; on voit souvent des parasites remplir presque complètement un blastophore sans que le noyau des cellules supportées par 
ce blastophore présente la moindre trace d'altération; la figure 153 montre de tels noyaux normaux dans des spermatides dont le cytoplasme commence à s'étirer (comparer avec fig. 155 qui représente des spermatides normales).

Il y a ensuite condensation rapide de la chromatine. On constate d'abord une hyperchromatose du réseau puis, il y a fusion des divers filaments gonflés de ce réseau en une masse plus ou moins compacte ; c'est ici encore un processus tout à fait comparable à la pycnose, mais on peut aussi le considérer comme une accélération de développement qui amène tout de suite la chromatine à l'état où on l'observe dans les spermatides normales à noyau condensé. La pycnose qui est un processus de dégénérescence produit naturellement un changement notable dans la nature de la chromatine (cf. BouIn 1897), et l'altère profondément. Nous ne trouvons pas ici d'altération semblable. Sans doute si l'envahissement parasitaire a eu lieu au début des spermatocytes de deuxième ordre, ou un peu plus tard, dans le cas d'un blastophore peu volumineux, la chromatine ne dépasse pas ce stade de condensation, elle reste à l'état de boule arrondie, compacte, colorée en bleu par la méthode de Mann. Mais si, au contraire, l'envahissement a été un peu plus tardif, l'évolution de cette chromatine continue ; elle reste massive, mais elle peut s'allonger légèrement, devenir piriforme, naviculaire ou prendre la forme d'un très court cylindre. De plus, avec l'aide du Mann, on constate sur elle tous les changements de colorabilité que nous avons observés sur le noyau normal des spermatides se transformant en spermatozoïdes, et finalement, elle se colore en rouge vif. Ce n'est donc pas de la chromatine morte ou en dégénérescence, puisqu'elle subit les mêmes transformations chimiques que la chromatine normale.

Ces mêmes changements de colorabilité se retrouvent dans les divers phénomènes dégénératifs qui nous restent à décrire et qui sont causés par des parasites envahissant un blastophore couvert de spermatides. 
Dans ces cas encore, nous constatons une condensation de la chromatine nucléaire qui se produit simplement alors par un tassement des grains chromatiques disséminés dans le noyau, ce qui donne lieu à la formation de masses fusiformes ou aciculaires. L'emploi du Mann, nous permet de constater que ces masses sont d'abord moniliformes comme les spermatides normales (HESsE 1909), avec une croûte superficielle bleu foncé et un centre bleu pâle ; puis le centre devient rouge et la périphérie est occupée par une couche discontinue de granulations d'un bleu intense, enfin, toute la masse chromatique se colore en rouge. On peut remarquer seulement que, alors que dans la spermatogenèse normale ces phénomènes de changement de colorabilité se produisent d'une façon synchrône pour les cellules d'une même spermatosphère, il n'en est plus de même dans les spermatosphères parasitées où certaines cellules se colorent encore en bleu, alors que d'autres se colorent en violet ou en rouge (fig. 187).

Il est intéressant de remarquer que ces masses chromatiques ne deviennent jamais filiformes comme les spermatozoïdes normaux ; ceci est dû sans doute à l'action centripète exercée par le parasite sur la chromatine. Dans certains cas d'envahissement très tardif, la spermatide achève presque ses métamorphoses, mais elle ne donne jamais un spermatozoïde cylindrique; il y a toujours légère accumulation de chromatine dans la région voisine du blastophore et l'épaisseur du filament va en diminuant à mesure que l'on se rapproche de son extrémité distale. Cette extrémité est aussi le plus souvent légèrement courbée, de même que l'extrémité proximale mais en sens inverse, de façon à figurer un $\mathrm{S}$ à courbures plus ou moins accentuées (fig. 168). C'est là une disposition qui ne s'observe pas dans le spermatozoïde normal. Nous avons représenté (fig. CIII et fig. 168, 169, 170) divers aspects de ces spermatozoïdes que l'on peut comparer aux spermatozoïdes normaux (fig. 167).

Dans ces derniers cas, les éléments sexuels altérés restent 
fixés à la paroi du blastophore jusqu'à ce que le parasite brise son enveloppe et l'abandonne.

Lorsque l'étirement de la chromatine n'a pu se produire et que le cytoplasme des spermatides est absorbé par la Grégarine, on voit les grains chromatiques condensés abandonner leur situation radiale, ils se rapprochent de la surface du parasite (fig. 141), s'appliquent étroitement sur cette surface et parfois se rassemblent en certains points en paquets situés entre le parasite et la membrane blastophorienne. Le plus souvent ils finissent par se réunir tous en une masse compacte en avant de la ventouse (fig. 142), appliquée contre la membrane qui entoure le blastophore et séparée de la ventouse par un espace vide plus ou moins volumineux. Ceci est très facilement visible sur des frottis observés in vivo.

Il semble qu'il y ait là une véritable aspiration du cytoplasme des cellules sexuelles qui viendrait à la ventouse et serait absorbé par le parasite; les blocs de chromatine condensée, restes des noyaux le suivraient dans cette migration mais ne seraient pas absorbés, de là leur accumulation en face de la ventouse où ils restent jusqu'à ce que le parasite quitte son enveloppe.

En somme, toutes les altérations produites par les divers parasites sur les cellules sexuelles de l'hôte peuvent se résumer en quelques lignes:

$1^{0}$ Formation d'une membrane résistante qui entoure le parasite ;

$2^{\circ}$ Destruction du blastophore et distension souvent énorme de ce blastophore ou plutôt de la membrane qui l'enveloppe.

$3^{\circ}$ Attraction des noyaux des cellules sexuelles du follicule spermatique envahi et excitation de ces noyaux à la multiplication;

$4^{\circ}$ Accélération du développement qui se traduit par un étirement précoce du cytoplasme et une condensation rapide de la chromatine, comparables à ce qui se produit à la fin de 
l'histogenèse du spermatozoïde, mais ne donnant jamais que des produits abortifs et stériles.

\section{Défense de l'Hôte.}

Nous avons vu, en étudiant le développement des parasites comment se produisait dans la nature la dissémination et la propagation des Monocystis.

Un grand nombre de germes doivent périr sans pouvoir se développer, soit qu'ils restent longtemps à l'extérieur avant d'être ingérés par un nouvel hôte, soit même qu'ils soient détruits avant d'être mis en liberté. Nous avons vu en effetque si les parasites ne sont pas attaqués en général par les phagocytes de l'hôte durant leur vie végétative, ils sont de suite entourés au contraire quand ils passent à l'état de kystes. Longtemps ces kystes restent inaltérés, le développement des sporocystes se poursuit dans leur intérieur sans subir aucun trouble appréciable et ils arrivent à la maturité ; cependant, à la longue, un certain nombre de kystes sont disloqués, les phagocytes pénètrent dans leur intérieur, s'emparent des sporocystes isolés qui résistent aussi très longtemps à leur attaque mais finalement se ratatinent, se flétrissent et sans nul doute sont digérés. On trouve ainsi, surtout chez les Helodrilus de nombreux sporocystes en dégénérescence dans les nodules phagocytaires brunâtres des vésicules séminales et du cœlome.

Sans doute une partie des parasites est encore détruite au moment de leur migration à travers les parois intestinales et le cœlome pour gagner les vésicules séminales. Ainsi l'action défensive de l'hôte se manifeste à la fois sur le sporocyste et sur le jeune sporozoïte. Malgré cela nous avons vu que presque tous les Oligochètes sont infestés. Nous avons vu aussi que malgré la quantité souvent énorme de parasites que contiennent leurs vésicules séminales, les Oligochètes restent fertiles, cela tient sans doute en grando partie à ce que les testicules 
eux-mêmes ne sont jamais envahis, le parasite n'attaquant la cellule sexuelle que lorsqu'elle s'est détachée de ce testicule. Ainsi celui-ci reste indemne et fournit constamment de nouveaux éléments dont quelques-uns échappent à l'action parasitaire et suffisent à assurer la fécondité de l'hôte.

Simdlecki (1903) chez la Polymmie et Brasil (1905 c) chez le Lombric ont montré que les phagocytes attaquaient non seulement les éléments parasitaires, mais encore qu'ils jouaient un grand rôle dans le nettoyage des vésicules séminales après les émissions spermatiques, se saisissant des éléments reproducteurs mal formés ou inutilisés et des blastophores dénudés, et les digérant.

Ils s'emparent également des

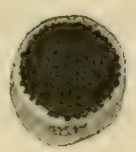

$$
1 \mathrm{~V}
$$

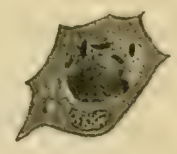

FIG. UIV. Amas de cornichons chromatiques abandonnés par St. coronota et englobés par un phagocyte de l'hóte. Ils se sont tassés fortement $x$ 1.300. - FIG. CV. Quelques grains chromatiques, restes de cellules sexuelles, abandomnés par $S t$. coronota et saisis par un phagocyte $\times 1.300$.

débris abandonnés par les parasites : cellules sexuelles altérées, recouvrant les enveloppes blastophoriennes flétries et rejetées par les Grégarines (fig. clv et cv).

Ainsi se trouvent récupérées une grande quantité de matières qui seraient perdues pour l'organisme de l'hôte.

\section{CONCLUSIONS}

Toutes les Grégarines signalées jusqu'ici chez les Oligochètes sont des Monocystidées. Ces parasites sont très répandus, on en a observé chez toutes les familles d'Oligochètes sauf les Naïdides et les Aelosomides. De tous les Oligochètes que nous avons examinés, seul Eiseniella tetraedra Sav. s'est toujours montré indemne de Grégarines.

Les Monocystidées des Oligochètes habitent quelquefois 
l'intestin, mais on les rencontre plus particulièrement dans la cavité générale et les vésicules séminales de l'hôte. Les formes intestinales sont en effet rares; par contre les espèces coelomiques sont très abondantes; la plupart de ces dernières se donnent rendez-vous dans les vésicules séminales où elles trouvent une nourriture abondante fournie par les cellules sexuelles en voie de développement; les espèces habitant uniquement la cavité générale sont peu nombreuses.

La spécificité parasitaire de ces Monocystidées ne paraît pas très étroite; beaucoup d'entr'elles en effet attaquent indifféremment des hôtes appartenant à diverses espèces d'un même genre ( $M$. lumbrici, $M$. agilis, $Z$. cometa, $S t$. coronata) ; parfois même à des espèces de genres différents ( $M$. herculea) ; quelques-unes cependant paraissent étroitement spécialisées : $N$. magna ne se rencontre que chez $L$. herculeus, $M$. hirsuta que chez $L$. castaneus, etc.

Chez une même espèce d'Oligochète, on rencontre parfois des parasites différant avec l'habitat : ainsi $H$. caliginosus Sav. donne asile en Algérie à $M$. Le Mêmei que l'on ne rencontre pas dans les individus de la même espèce provenant de la France continentale.

Les Monocystidées des Oligochètes présentent une grande variété de formes, ce qui permet de les ranger en sept genres différents, dont deux seulement s'écartent de l'ensemble par la forme de leurs sporocystes, la distinction des autres étant uniquement basée sur les caractères morphologiques du trophozoïte. Ces formes peuvent être considérées comme dérivant de deux types extrêmes l'un globuleux, l'autre nématoïde. Malgré cette grande variété d'aspect des états végétatifs, les sporocystes sont tous construits sur le même type et ne diffèrent guère que par leurs dimensions si l'on met à part les deux genres Schaudinnella et Urospora et deux espèces appartenant à d'autres genres : M. Duboscqi et St. coronata. 
Cette uniformité nous permet de conclure à une parenté assez étroite entre tous ces parasites.

L'origine du groupe serait sans doute à chercher dans les Pseudo-monocystidées des Polychètes, les Doliocystis par exemple, que Brasil (1908) considère déjà comme une étape vers les Monocystidées cœlomiques des Polychètes mêmes. Les espèces du genre Rhynchocystis présentent en effet de véritables épimérites, et celui de $R$. pilosa est fort comparable à celui de Doliocystis aphroditae. L'épimérite des Stomatophora, de St.coronata en particulier est aussi compliqué que celui de bien des Cephalina et comme ces derniers, il est caduc. Les Stomatophora et les Rhynchocystis serviraient ainsi d'intermédiaires entre les Pseudononocystidées et les Monocystidées typiques. Chez ces dernières, il n'y a pas trace d'épimérite ou bien celui-ci est simplement représenté par un rostre hyaloplasmique un peu plus développé que celui du sporozoïte.

La cuticule montre des ornementations variées : stries longitudinales et transversales, mamelons arrondis ou poils d'épaisseur diverse, filiformes ou en lanières. Le sarcocyte n'est jamais très développé, il est quelquefois disposé en bandes longitudinales plus épaisses séparées par des sillons. Les fibrilles du myocyte sont faciles à apercevoir en général, chez quelques espèces cependant nous n'avons pas réussi à les mettre en évidence. Les Nematocystis ont à la fois des fibrilles longitudinales et des fibrilles transversales, il en est de même d'autres espèces très agiles comme $M$. lumbrici.

L'aspect de l'endoplasme est variable, les différences sont dues surtout à la forme et au volume des grains de paramylon logés dans les alvéoles et à la forme des granulations chromatoïdes qui siègent dans les travées de spongioplasme.

La structure du noyau est très simple chez la plupart des espèces. Il existe en général un karyosome et un réseau de linine sur lequel sont répartis des grains chromatiques. Parfois le réseau est absent et toute la chromatine nucléaire est contenue 
dans le karyosome. Celui-ci, simple ou multiple donne naissance par bourgeonnement à des grains chromatiques qui tombent dans le suc nucléaire ou qui, parfois, passent directement dans le cytoplasme. La structure de ce karyosome est en général simple ; c'est une masse dont le centre est vacuolaire et formé de plastine, la croûte périphérique se colorant au contraire comme la chromatine; cependant il présente parfois des couches alternativement colorées comme de la chromatine et comme de la plastine et peut envoyer dans tout le noyau des ramifications arborescentes qui vont s'accoler à la membrane nucléaire $(P$. Cuenoti). On rencontre chez certaines espèces deux karyosomes, l'un chromatique, l'autre nucléinien et qui paraissent provenir l'un de l'autre par bourgeonnement.

Le karyosome semble jouer un rôle important durant la vie de la Grégarine. Les recherches de Cú́not (1901) BrasiL (1905) ont montré qu'il disparaît au contraire au début des processus de la sexualité.

La plupart des espèces ont des sporocystes à parois très résistantes, susceptibles de vivre longtemps au dehors sans subir d'altération. Ces sporocystes mûrs s'ouvrent sous l'action des sucs digestifs de l'intestin moyen s'ils sont absorbés par un hôte approprié. Cette déhiscence est bipolaire ; les sporozoïtes s'échappent par un étroit orifice formé à chaque pointe du fuseau sporocystal. Ils gagnent les vésicules séminales sans doute très rapidement, car nous n'avons pu suivre leur migration. Chez M. Duboscqi, les sporocystes ont toujours une enveloppe très mince, parfois même cette enveloppe manque et les sporozoïtes sont à nu dans l'intérieur du kyste. La paroi de celui-ci, très résistante, leur sert alors d'enveloppe protectrice.

Certaines espèces nous montrent assez souvent des altérations dont la cause première est inconnue. On observe ainsi divers phénomènes dégénératifs: hypertrophie considérable ou métamérisation du corps, dégénérescence hyaline ou granu- 
leuse du cytoplasme, plasmarrhexis, caryorrhexis ou pycnose du noyau ; parfois esquisses de mitoses dans les trophozoites non accouplés.

Outre les Metchnikovella et les Microsporidies, qui ont été signalées comme parasites des Grégarines, il convient de citer comme attaquant ces organismes des Bactéries spécifiques qui les envahissent et en provoquent la dégénérescence et la mort.

L'action sur l'hôte est très sensible et particulièrement intéressante pour les espèces dont une partie du développement est intracellulaire.

$N$. magna habite les pavillons ciliés et est suspendue à la paroi de ces pavillons de la même façon que certaines Polycytisdées aux cellules épithéliales de l'intestin. Elle détermine une hypertrophie considérable de la cellule qui la supporte et à l'intérieur de laquelle s'est effectué le début de son développement. Il apparaît en outre dans le cytoplasme de cette cellule-hôte des fibrilles très différenciées qui sont sans doute des racines ciliaires hypertrophiées et modifiées de façon à supporter une traction considérable. Les racines de ces fibrilles, qui se prolongent à la face interne de la cellule en s'intriquant pour constituer la basale, sont aussi fortement hypertrophiées et constituent des sortes de racines fixatrices.

La cellule-hôte prend la forme d'une cupule très développée à l'intérieur de laquelle est étroitement enchâssée l'extrémité antérieure du parasite.

Une telle formation cupuliforme se retrouve enserrant les pôles antérieurs des couples de $P$. Cuenoti ; mais ici, cette formation est pluricellulaire, chacune des cellules qui la composent présentant les mêmes modifications que la cellule cupuliforme unique de $N$. magna

Les espèces qui parasitent les blastophores produisent sur les cellules sexuelles supportées par ces blastophores des 
altérations fort variées et dont les variations sont en rapport d'une part avec le moment de la pénétration d'autre part avec la nature du parasite.

La Grégarine semble exercer d'abord une action attractive sur le noyau des cellules sexuelles environnantes et une action répulsive sur leur cytoplasme. Puis le cytoplasme est attiré à son tour et souvent absorbé. Le noyau n'est jamais absorbé, il subit des altérations fort variées, et qui pourraient, en dernière analyse, se ramener à une sorte de pycnose, mais que nous considérons plutôt comme une accélération de développement suivie d'un arrêt brusque à divers stades de ce développement. Ainsi se constituent des produits sexuels avortés qui sont abandonnés par le parasite au moment où il s'échappe du blastophore et englobés et digérés par les phagocytes de l'hôte en même temps que les sporocystes altérés et que les Grégarines en voie de dégénérescence.

\section{INDEX BIBLIOGRAPHIQUE}

1908. Awerinzew (S.). Ueber die Gregarinen aus dem Darme von Amphiporus sp. (Zool. Anz. T. XXXIII).

1884. Batbiani (G.). Leçons sur les Sporozoaires, in $8^{\circ}$, Paris.

1888 Beddard (F.-E.). Note on a new Gregarine (Proced. of the Zool. Soc. London).

1889. BEDdARD (F.-E.). On a new Sporozoon from the vesiculae seminales of Perichaeta. (Zool. Jahrb. Abt. f. sys. Bd. IV, Heft 4).

1895 BEDDARD (F.-E.). A Monograph of the order of Oligochaeta (Oxford), (Clarendon press).

1872 BenEDEN (E. van). Note sur la structure des Grégarines. (Bull. de l'Acad. Roy. de Belgique 2e Sér. T. XXXIII.)

1909. Bernard (N.). L'évolution dans la symbiose. Orchidées et leurs champignons commensaux. (An. Sc. nat. Bot. Série IX, Tome 9.)

1902. BeRNDt (A.). Beiträg zur Kenntnis der im Darme der Larve von Tenebrio molitor lebenden Gregarinen. (Arch. f. Protistenkunde Bd. I). 
1894 Bosanquet (W.-Cecil.). Notes on a Gregarine of the Earthworm (Lumbricus herculeus). (Quart. Journ. of. micr. Sc., $2^{\mathrm{e}}$ série, Vol. XXXVI).

1897. Bodin (P.). Etudes sur l'évolution normale et l'involution du tube séminifère. (Arch. d'Anat. microsc. T. I).

1904. Brasir (L.). Contribution à la connaissance de l'appareil digestif des Annélides Polychètes. L'épithélium intestinal de la Pectinaire. (Arch. de Zool. exp. et gén. (4) T. II.)

1905 Brasic (L.). Recherches sur la reproduction des Grégarines mono. cystidées. [Arch. Zool. exp. (4), T. III].

$1905 b$. Brasil (L.). Nouvelles recherches sur la reproduction des Grégarines monocystidées. [Arch. Zool. exp. (4) T. IV].

1905 c. Brasil (L.). La résorption phagocytaire des éléments reproduc-

- teurs dans les vésicules séminales du Lumbricus herculeus Sav. (C. R. Acad. Sc., Paris, T. CXIs).

1908. Brasit (L.). Le genre Doliocystis Léger (C.R.Acad. Sc. Paris, Tome CXLVI).

1883 84. Brass (A.). Biologische Studien. II Die Organisation der Thierischen Zelle. Halle.

1849. BRuch (G.). Einige Bemerkungen über die Gregarinen. (Zeitsch. f. wissensch. Zool. Bd. I).

1905 Bugnion (E.). et N. Popoff. La spermatogenèse du Lombric terrestre. [Arch. Zool. exp. et gen.(4), T. III]

1881. Bütschli (O.). Kleine Beiträge zur Kenntniss der Gregarinen. (Zeitschr. f. wiss. Zool. Bd. XXXV).

1882. Büтschli (O.). Sporozoa. (Bronn's Thierreich Bd. I. Abth. I, Leipzig 1880-82).

1897. Gauliery (M.) et F. Mesnil. Sur un type nouveau (Metchnikovella. n. g.) d'organismes parasites des Grégarines [C.R.Ac. Sc., Paris, T. GXXV et C. R. Soc. Biol. Paris (10), T. IV].

1898 Caullery (M.) et F. Mesnil. Les formes épitoques et l'évolution des Cirratuliens (Annales de l'Univ. de Lyon T. XXXIX).

1899. Cauldere (M.) et F. Mesnil. Sur quelques parasites internes des Annélides. (Travaux Stat. Zool. Wimereux Vol. 7).

1905. Cauliery (M.) et F. Mesnil. Recherches sur les Haplosporidies. [Arch. Zool. exp. et gén. (4), T. IV].

1902. Cecconr (J.). De la sporulation de la Monocystis agilis Stein. (Arch. d'Anat. microsc. T. V).

1861. Claparède (A.-René-Ed.). Etudes anatomiques sur les Annélides, Turbellariés, Opalines et Grégarines observés dans les Hébrides (Mém. de l. Soc. phys. et d'hist. nat. Genève, T. XVI). 
1895. CLARKe (J.-Jackson). Observations on various Sporozoa (Quart. Journ. of micr. Sc. 2e sér. Vol. XXXVII).

1907. Cones (Salvatore). Untersuchungen über den Chromidialapparat der Gregarinen. (Arch. f. Protistenkunde Bd. X).

1898 Cú́хот (L.). Etudes physiologiques sur les Oligochètes. (Arch. de Biologie. T. XV).

1901. Cú́xot (L.). Recherches sur l'évolution et la conjugaison des Grégarines. (Arch, de Biologie, T. XVII, fasc. 4).

1902 Cúnот (L.). Legerella testiculi nov. sp., Coccidie parasite du testicule de Glomeris. (Arch. Zool. exp. et gén. (3), T. X Notes et Revue, $\mathrm{n}^{\text {os }} 3$ et 4 ).

1902 b. Cú́not (L.). Organes agglutinants et organes cilio-phagocy taires. [Arch. Zool. exp. et gén. (3), T. X].

1896 Defaye (Y.) et E. Hérodard. Sporozoaires in Traité de Zoologie concrète, I. Protozoa. Paris (Schleicher).

1906. Depdol.LA (Ph.). Beiträge zur Kenntniss der Spermatogenese beim Regenwurm. (Lumbricus terrestris L. Müll), (Zeitsch. f. viss. Zool. Bd. LXXXI).

1901. Doflein (F.). Die Protozoen als Parasiten und Krankheitserreger. Iéna (Gustave Fischer).

1906. Dogiel (V.). Beiträge zùr İenntniss der Gregarinen. (Arch. f. Protistenkunde $\mathrm{Bd}$. VII.)

1907. Dogres (V.). Beiträge zur Kenntniss der Gregarinen. II Schizocystis sipunculi nov. sp. (Arch. f. Protistenkunde Bd. VIII).

1893. Drüner (L.). Beiträge zur Kenntniss der Kern-und Zell-degeneration und ihrer Ursachen. (Micrococcidium cariolyticum), in den Spermatocyten und im Darmepithel von Salamander und Triton), (Jenaische Zeitschrift, Bd. XXVIII).

1903. Drzewecki (IV.). Ueber vegetative Vorgänge im Kern und Plasma der. Gregarinen der Regenwurmshodens. (Arch. f. Protisten. kunde. $\mathrm{Bd}$. III).

1907 a. Drzewetzky (W.-F.). Ueber bewimperte Gregarinen (en Russe), (Tras. Soc. nat. C. R. séances Saint-Pétersbourg).

$1907 b$. Drzewecki (W.). Ueber vegetative Vorgänge im Kern und Plasma der Gregarinen II Stomatophora coronata nov. gen. (Arch. f. Protistenkunde Bd. X).

1828. DUGÉS. Recherches sur la circulation, la respiration et la reproduction des Annélides Abranches. (Ann. Soc. Nat. T. XV).

1835 DUJARDin (F.). Recherches sur les organismes inférieurs. II. Sur les Infusoires appelés Protées. (Annales des $s c$. natur. $2^{\mathrm{e}}$ série 'Lool. 'T. IV, 1). 
1845 DuJARdin (F.). Histoire naturelle des Helminthes ou vers intestinaux. In $8^{\circ}$, Paris 1845. Appendice I. Helminthes dont la place est incertaine. Gregarine.

1895 EIsen (Gust.). On the various stages of development of Spermatobium n. g., with notes on other parasitic Sporozoa. (Proced. of the California Acad. of Sc., 2e série, Vol. V).

1902. Féltzet (G.) et Al. Branca. Recherches sur le testicule en ectopie. (Journ. Anat. Physiol. Paris. Ann. 38).

1848 Frantzius (Alex. v.). Einige nachträgliche Bemerkungen über die Gregarinen. (Arch. f. Naturg. Iahrg. 14. Bd. 1).

1876 GABRIEI, (B.). Uber Entwickelungsgeschichte der Gregarinen. (53 Iahreshericht d. schles. Gesellsch. f. vaterl. Kultur. i. I. 1875. Breslau, 1876).

1877. Gabriel (B.). Zur Entwickelungsgeschichte der Gregarinen (Amtlicher Bericht d. 50. Versamml. deutscher Naturf. u. Arzte in München).

1878 GaBriel Ueber einige Umbildungen der Pseudonavicellen. (Iahresber. der schles. Gesellsch. f. vaterl. Cultur. [f. d. 1. 1877. Breslau, 1878).

1879. GabRiel Ueber primitives Protoplasma. (Iahresber. der schles. Ges. f. vaterl. Cultur. f. d. I. 1878. Breslau, 1879).

1880. Gabries (B.). Zur Glassification der Gregarinen. (Zool. Anzeiger T. III).

1898. Giard (Alfred.). Sur la synonymie et la géonémie de Microscolex phosphoreus Dugès. (C. R. Soc. de Biol. Paris. T. V, $10^{\mathrm{e}}$ série).

1880. Greef (R.). Die Echiuren (N. Acta d. K. K. Leopold Akad. Bd. 41).

1835. Hente. Uber die Gattung Branchiobdella (Müllers's Archio.).

1845. Hente (J.). Uber die Gattung Gregarina. (Müllers'Archiv.f. Anat. Phys. u. (viss. Med.).

1888. Hennegur (F.). Formation des spores de la Grégarine du Lombric. (Annales de Micrographie, T. I).

1894 Henneguy (F.). Recherches sur l'atrésie des follicules de Graaf chez les Mammifères et quelques autres Vertébrés. (Journ. de l'anat. et de la phys., T. XXX).

1904 Hesse (E.). Monocystidée nouvelle des Pheretima. (Bulletin mensuel de l'Association française pour l'avancement des sciences no 9 , novembre 1904).

1905. Hesse (E.). Sur Myxocystis Mrazeki Hesse, Microsporidie parasite de Limnodrilus Hoffmeisteri Clap. (C. R. Séances Soc. Biol., T. LVIII). 
1909. Hesse (E.). Quelques particularités de la Spermatogenèse chez les Oligochèles. [Arch. Zool. exp. et gén. (4), T. X, no 2].

1908. HoFFMAN (Richard). Uber Fortpflanzungsercheinungen von Monocystideen der Lumbricus agricola. (Arch. f. Protistenkunde Bd. XIII).

1842. Hoffmeister. De vermilius quibusdam ad Genus Lumbricorum pertinentibus Dissertatio. Berolini.

1823 Home (Sir. E.). On the Double Organs of Generation of the Earthworm, etc. (Phil. Trans.).

1895. Israíl et Pappenheim. Ueber die Entkernung der Säugethiererythroblasten. (Arch. f. path. Anat. Bd. CXLIII).

1848. KöLliker (A.). Beiträge zùr Kenntniss niederer Tiere I. Ueber die Gattung Gregarina. (Zeitsch. f. wiss. Zool. Bd. I).

1892 KonotNeFF (A.). Myxosporidium bryozoides. (Zeitschrift für wiss. Zool. Bd. LIII).

1896 Kowalewsky. Sur les glandes lymphatiques des Néréides. (C. R.

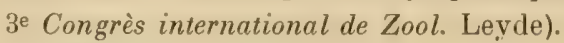

1907 KUnze (Wilhelm.). Uber Orcheobius herpobdellae Schuberg et Kunze ein Coccidium aus Herpobdella atomaria Car. (Nephelis vulgaris Moq. Tand.) (Arch. f. Protistenkunde Bd. IX).

1907. KuschaKewitsci (S.). Beobachtungen über vegetative, degenerative und germinative Vorgänge bei den Gregarinen des Melhwurmdarms. (Arch. f. Protistenkunde Suppl. I).

1899 Labbé (A.). Sporozoa. (Das Thierreich, 5 Lief. in-8o, Berlin).

1863. Lankester (E. Ray.). On our present Knowledge of the Gregarinidae, with Descriptions of three new Species belonging to that class. (Quart. Journ. of microsc. sc. N. Sér., T. III).

1872. LANKESter (R.). Remarks on the structure of the Gregarinae and on the development of Greg. (Monocystis) Sipunculi Köll. (Quart. journ. of micr. Sc. N. Sér. T. XII.)

1900. Laveran (A.) et F. Mesnil. Sur quelques particularités de l'évolution d'une Grégarine et la réaction de la cellule-hôte. (C. R. Soc. Biol. Paris, T'. LII).

1892 Léger (L.). Recherches sur les Grégarines. (Tablettes zool. T. III).

1897. Léger (L.). Contribution à la connaissance des Sporozoaires parasites des Echinodermes. (Bull. sc. de la France et de la Belgique T. $\mathrm{XXX)}$.

1899. Léger (I...). Sporozoaires du tube digestif de l'orvet. (C.R. Ac. Sc. Paris, T. CXXVIII).

1901. LÉGER (L.). Sur une nouvelle Grégarine des Pinnothères des Moules. (C. R. Acad. Sc. Paris, T. GXXXII). 
1904 Léger (L.). La reproduction sexuée chez les Stylorhynchus. (Archio. f. Protistenkunde, Bd. III)

1906 LÉGER (L.). Etude sur Taeniocystis mira Léger, Grégarine métamérique (Arch. f. Protistenkunde, Bd. VII;).

1907. LÉGER (L.). Les Schizogrégarines des Trachéates I. Le genre Ophryocystis. (Archiv. f. Protistenkunde, Bd. VIII).

1908. LÉGER (L.). Mycétozoaires endoparasites des Insectes I Sporomyxa scaurin. g., n. sp. (Arch. f. Protistenkunde Bd. XII).

1899 LÉger (L.) et O. Dubosce. Notes biologiques sur les Grillons. B Gregarina.Davini n. sp. (Arch. Zool. Exp. et gén. Notes et Revue (5), T. VII).

1902 LÉGer (L.). et O. DuBosce. Les Grégarines et l'épithélium intestinal chez les Trachéates. (Arch. de Parasit. T. G).

1903 LÉGer (L.) et O. Dubosce. Recherches sur les Myriapodes de Corse et leurs parasites. [Arch. Zool. exp. et gén. (4) T. I].

1904 LÉger (L.) et O. Dubosce. Nouvelles recherches sur les Grégarines et l'épithélium intestinal des Trachéates. (Arch. $f$. Protistenkunde $\mathrm{Bd}$. IV).

1908. Léger (L.) et O. DuboscQ. L'évolution schizogonique de l'A ggregata (Eucoccidium) eberthi(Labbé). (Arch.f. Protistenkunde, Bd. XII).

1909. Léger L.) et O. DuboscQ. Sur une Microsporidie parasite d'une Grégarine. (C. R. Acad. Sc. Paris. T. CXLVIII, no 11, 15 mars).

1909. Léger (L.) et O. Dubosco. Etudes sur la sexualité des Grégarines (Arch. f. Protistenkunde Bd. XVII).

1882 LeIDy (Jos.). On Enchytraeus, Distichopus and their parasites. (Proced. of the Acad. of Nat. Sci. Philadelphia).

1855 Lreberkühn (Nath.). Evolution des Grégarines. (Mém. cour. et mém. des sav. étrangers de l'Acad. de Belgique, T. XXVI).

1865 LIEBERKüHN (Nath.). Beitrag zur Kenntniss der Gregarinen (Archiv. f. Anat. Physiol. u. wiss. Med. Berlin).

1904 LüHE. Bau und Entwickelung der Gregarinen.I Teil : Die Sporozoîten, die Wachstumperiode und die ausgebildeten Gregarinen. (Arch. f. Protistenkundé Bd. IV).

1907. Marcozzi (V.). Action des poisons de la tuberculose sur le parenchyme du. testicule. (Annales des maladies des organes génito. urinaires. Juillet, no 13).

1844. MEcKeL (H.). Ueber Geschlechtsapparat einiger hermaphroditen Thiere. (Archiv. für Anatomie, Physiologie und wissenschaftliche Médicin Berlin I.)

1845. Menge (A.). Zur Rotwürmergattung Euaxes. (Arch. f. Naturg. XI Iahr., Bd. I). 
1959 Mesnil (F.). Article de critique in (Bulletin de l'Institut Pasteur, T. VII, no 1).

1892 Metchnikoff (E.). Leçons sur la pathologie comparée de I'inflammation. Paris.

1900. Michatensen. Oligochaeta. (Das Thierrheich. 10 Lieferung. Berlin).

1903. Minchin (E.-A.). The Sporozoa in Trealise on Zoo'ogy (Lankester) Part I. Second Fascicle. London. (Adam et Charles Black).

1892. Mingazzivi (P.). Contributo alla conoscenza dei Coccidi. (Atti d. R. Accad. d. Lincei. Rendiconti della classe di Scienze fisiche, matematische e naturali Roma 4, sér. 5, V. I.Sem. I).

1823 Montèqre. Observations sur les Lombrics ou Vers de terre. (Mémde Mus. I).

1906 Moroff (Th.). Bemerkungen über den Kern der Aggregata Frenzel. (Zool. Anz. Bd. XXXI, no 2, 3).

1907. Moroff (Th.). Nucleolen, Karyosomen und ihre Funktion. (Zentralblatt für Physiologie, Bd. XXI, no 6).

1908 Monoff (Th.). Die bei den Cephalopoden vorkommenden Aggregataarten als Grundlage einer kritischen Studie über die Physiologie des Zellkerns. (Arch. f. Protistenk. Bd. XI).

1826 Morren (Carol.-F.-A.). Respontio ad questionenn, etc. : Quaeritur descriptio structurae anatomicae et expositio historio-naturalis Lumbrici vulgaris sive terrestris. (Ann. Ac. Gandavensis. Gandavi 2 octobre 1826).

1899. Mrazek (A.). Studia o Sporozoīch. I. Dèleni jaderné a sporulace u Gregarin. (Studien an Sporozoen. I Kernteilung und Sporulation bei den Gregarinen) in-80 Prag (Véstnik kral ceské spolecnosti nauk, Tr. math-prirod 1899. no 25).

1882 NAsse (D.). Beiträge zur Anatomie der Tubificiden. (Dissert. inaug. in- $4^{\circ}$. Bonn.).

1905 Nereshetmer (Eugen). Uber vegetative Kernveränderungen bei Amoeba Dofleini nov. sp. (Archiv. für Protistenkunde $\mathrm{Bd} \mathrm{VI}$ ).

1886 Neuland (G.). Ein Beitrag zur Kenntniss der Histologie und Physiologie der Generationsorgane des Regenwurmes. (Verh. des naturhistorischen Vereins der preussischen Rheinland. Bonn. Bd. XLIII).

1903 Nusbaum (Josef). Ueber die geschlechtliche heterogame Fortpflanzung einer im Darmkanale von Henlea leptodera Vejd. schmarotzenden Gregarine, Schaudinnella henlea mihi. (Zeitschrift f. wiss. Zool. Bd. LXXV, Heft 2). 
1881. Perrier (Edm.). Etudes sur l'organisation des Lombriciens terrestres. - IV. Organisation des Pontodrilus (e. p.). - Parasites des Pontodriles. (Arch. Zool. Exp., T. IX).

1891. PFEIFFER (L.). Die Epithelzellen-und Spermamutterzellen. Infektion im Hoden des Regenwurmes durch Monocystiden. (Die Protozoen als Kranheitserreger. In-80. Iéna).

1893 Pfeiffer (L.). Untersuchungen über den Krebs. Die Zellerkranhungen und die Geschwulstbildunger durch Sporozoen. In- $8^{\circ}$. Iéna.

1895 Pfeiffer (L.). Zur Verbreitung der Glugeaparasiten im Thierreich. (Die Protozoen als Krankheitserreger. Nachtrag. Iéna).

1897. Porter (James-F.). Two new Gregarinida. (Journ. of. Morphology. T. XIV, no 1).

1908. PoRter (A.). A new Schizogregarine, Merogregarina amaroucii nov. gen., nov. sp., parasitic in the alimentary tract of the composite Ascidian Amaroucium sp. [Arch. Zool. expér. (4), T. IX, Notes et Revue).

1902. Prowazek (S.). Zur Entwickelung der Gregarinen. (Arch. f. Protis. tenkunde, $\mathrm{Bd}$. I).

1896 Racovitza (Emile-G.). Le lobe céphalique et l'encéphale des Annélides Polychètes. (Anatomie, Morphologie, Histologie). (Arch. Zool. exp. et gén. (3) 'T. IV).

1870. RADKeWITSCH (G.). Ueber einen Parasiten aus Enchytraeus vermicularis (Gregarina enchytraei) (en Russe). (Travaux de la Société des Naturalistes à l'Université impériale de Kharkow. V. I).

1885. Roux (W.). Beiträge zur Entwickelungsmechanik des Embryo im Froschei. (Zeistch. f. Biologie XXI).

1885. Ruschнатрт (G.). Beitrag zur Entwickelungsgeschichte der monocystiden Gregarinen aus dem Testiculus des Lumbricus agricola. (Jenaische Zeitschr, f. Naturw. Bd. XVIII).

1894 ScheiwiakofF (W.). Ueber die Ursache der fortschreitenden Bewegung der Gregarinen. (Zeitsch. f. wiss. Zool. Bd. LVIII, Heft. 2).

1854 Sснмпт (Adolphe). Beitrag zur Kenntniss der Gregarinen und deren Entwickelung. (Abhandlg. d. Senckenberg. naturf. Gesellsch. Franckfurt. Bd. I, Heft. 1).

1875. SchneIder (Aimé.). Contributions à l'histoire des Grégarines des Invertébrés de Paris et de Roscoff. (Arch. Zool. exp. T. IV). 1899 Schneider (Guido.). Ueber Phagocytose und Exkretion bei den Anneliden, (Zeitschr. f. wiss. Zool. Bd. LXVI, 4). 
1906 Schuberg (August) und Wilhelm Kunze. Uber eine Coccidienart aus dem Hoden von Nephelis vulgaris.(Herpobdella atomaria), Orcheobius herpobdellae nov. gen., nov. sp. (Verhandlungen der Deutschen Zoologischen Gesellschaft, juin 1906 zu Marburg).

1899. Siedlegki (Michel). Ueber die geschtlechtliche Vermehrung der Monocystis ascidiae R. Lank. (Anz. d. Akad. d. Wiss. Krakau).

1901. SiedLeCKI (M.). Contribution à l'étude des changements cellulaires provoqués par les Grégarines (Arch. Anat. microsc. T. 4).

1902 Siedlecki (M.). Cycle évolutif de la Caryotropha Mesnili Coccidie nouvelle des Polymnies. (Bulletin de l'Ac. des Sc. Cracovie).

1903. Siedlecki (M.). Quelques observations sur le rôle des Amœbocytes dans le cœlome d'une Annélide. (Annales de l'Institut Pasteur, T. XVII, no 7).

1905. Siedlecki (M.). Ueber die Bedeutung des Karyosoms. (Bull. de l'Acad. d. Sc. Cracovie).

1907. SiedLecki (M.). Ueber die Struktur und die Lebensgeschichte von Caryotropha Mesnili). (Anz.d. Akad. d. Wissensch. Krakau).

1848 Stein (Fr.). Uber die Natur der Gregarinen. (Muller's Arch. f. Anat. Phys. u. wiss. Med.).

1904 Stempeli (W.). Ueber Nosema anomalum Monz. (Arch. f. Protistenk. Iéna. Bd. 4.).

1886 Suriray. Notice sur quelques parasites et produits organiques du Lombric terrestre. (Ann. des Sc. Natur, $2^{\mathrm{e}}$ série Zool. T. VI).

1884 TANGL (E.). Zur Lehre von der Continuität des Protoplasma. (Sitz. d. K.-K. Akad. d. Wiss. Wien XC).

1890. Thélohan (P.). Sur deux Coccidies nouvelles parasites de l'Epinoche et de la Sardine. (Annales de Micrographie, T. II).

1856 UdeKem (Jul. d'.). Développement du Lombric terrestre (Mém. cour. et mém. des sav. étrang. de l'Acad. de Belgique, T. XXX).

1876 Vejpovsky. Ueber Phreatothrix, eine neue Gattung der Limicolen. (Zeitsch. f. wiss. Zool. Bd. XXVII).

1896 Wastelewsky (V.). Sporozoenkunde. Iéna (Gustav Fischer).

1891. Wolters (Max:). Die Konjugation und Sporenbildung bei Grega. rinen. (Arch. f. mik. Anat. Bd. XXXVII).

\section{EXPLICATION DES PLANCHES}

PLANCHE I

La plupart des figures de cette planche ont été dessinées d'après des frottis fixés au sublim6. alcool et colorés par l'hématoxyline au fer et le mélange Litchgrün-éonine. La figure 8 seule . est reproduite d'après une coupe. 
Fra. 1. Mucron antérieur de Monocystis lumbrici non recouvert par l'ectoplasme ; stries épicytaires divergentes à partir de sa base; fibrilles du myocyte vues en coupo optique $\times 1300$.

FIG. 2. Touffe de poils postérieurs de $M$. lumbrici. Poils larges et arrondis $\times 1.300$.

FIG. 3. Touffe de poils de $M$. lumbrici. Poils enchevêtrés formant un feutrage $\times 1.300$.

FIG. 4. Touffe de poils de $\boldsymbol{M}$. lumbrici. Poils filiformes $\times 1.300$.

FIa. 5. Jeune $M$. lumbrici. Mucron antérieur, touffe de poils postérieurs, noyau à 2 karyosomss ; cytoplasme granuleux sans grains de paramylon $\times 1.300$

FIG. 6. $M$. lumbrici; noyau à 3 karyosomes, masses chromatoïdes dans le cytoplasme $\times 1.300$.

Fia. 7. M. lumbrici. La membrane nucléaire a disparu en certains points par où la chromatine passe directement dans le cytoplasme. Dans les travées de spongioplasme, masses chromidiales en fer de lance $\times 1.300$.

FIc. 8. M. lumbrici. Coupe. Ectoplasme montrant les fibrilles du myocyte; masses chromidiales en fer de lance dans le cytoplasme $\times 1.300$.

FIG. 9. M. lumbrici, Forme parasite de $L$. rubellus. Noyau à karyosomes nombreux $\times 1.300$.

FrG. 10, M. agilis. Genou des formes massives avec le mucron incolore $\times 1.300$.

Fit. 11. $\boldsymbol{M}$. agilis. Pied des formes allongées $\times 1.300$.

FIG. 12. M. agilis. Noyau à karyosome émietté $\times 1.300$.

FIt. 13. Jeune $\boldsymbol{M}$. agilis. Le cytoplasme renferme 1 grain chromidial accolé au noyau; sur la membrane nucléaire on voit un gros grain chromatique relié par un filament au karyosome $\times 1.300$.

FIG. 14. MI. agilis. Queue des formes allongées. Grain de paramylon géants $\times 1.300$.

Fra. 15. M. agilis. Parasite jeune. Noyau à 2 karyosomes autour desquels la chromatine est disposée en une rangée circulaire. Une grosse masse chromidiale dans le cytoplasme $\times 1.300$.

Fra. 16. $M$, agilis. Karyosome émettant des travées de grains chromatiques qui se répandent dans le noyau et passent même dans le cytoplasme par des orifices de la membrane nucléaire. Début du noyau flammé $\times 1.300$.

Fig. 17. $\boldsymbol{M}$. agilis. Cytoplasme dont les réserves ont presque complètement disparu. Karyosome bourgeonnant $\times 1.300$.

FIC. 18. M. agilis. Noyau au voisinage de l'ectoplasme. Karyosome presque totalement incolore. Grains chromatiques auréolés dans les travées de cytoplasme fortement colorées qui entourent le noyau $\times \mathbf{1 . 3 0 0 .}$

FIG. 19. Rhynchocystis pilosa. Epimérite allongé en trompe entourée par un manchon de sarcocyte orné de côtes sinuées et non velues. Noyau dans l'endoplasme épiméritique et présentant un prolongement antérieur $\times 1.300$.

Fio. 20. $R$. pilosa. Extrémité antérieure de la forme C. Manchon de sarcocyte strié autour du mucron. Poils à base élargie $\times 1.300$.

FIG. 21. R. pilosa. Epimérite en trompe conique entourée par sarcocyte, et ornée de poils à base élargie. Granulations sidérophiles dans la trompe, entre le mucron et le noyau $\times 1.300$.

FIG. 22. $R$. pilosa. Mucron épiméritique enfoui dans une cavité. Tout autour couronne de sarcocyte ornée de côtes radiaires $\times 1.300$.

FIG. 28. R. pilosa. Mucron saillant, Couronne de sarcocyte périmucronaire à côtes ondulées $\times 1.300$.

FIG. 24. $R$. pilosa. Mucron saillant. Couronne de sarcocyte périmucronaire soulevée tout autour en cratère. Karyosome avec grains accolés à sa surface $\times 1.300$.

FIG. 25. $R$. pilosa. Queue avec sa masse chromidiale caractéristique $\times 1.300$.

FIG. 26. $R$. pilosa. Noyau d'une forme jeune avec 2 karyosomes muriformes $\times 1.300$.

FIG. 27. $R$. pilosa. Poils à base élargies et renfermant du sarcocyte $\times 1.300$.

Fia. 23. $R$. porrecta. Mucron antérieur; noyau avec pointe antérieure, karyosome vacuolaire présentant seulement quelques points colorés à la surface $\times 1.300$.

Fia. 29. $R$. porrecta. Mucron antérieur; noyau avec karyosome accolé à la membrane dans la région opposée au mucron $\times 1.300$. 


\section{PLANCHE II}

Toutes les figures représentent des parasites fixés par le sublimé alcool et colorés par le Fer Lichtgrün-éosine (sauf les figures 41, 53, 54 : coloration à l'hémalun, et 51 coloration à la Safranine). FIG. 30. Rhynchocystis pilosa. Poils à base élargie renfermant de l'endoplasme $\times 500$.

FrG. 31. $R$. pilosa. Poils à base elargie avec cytoplasme granuleux renfermant des grains de paramylon $\times 1.300$.

FIG. 32. Monocystis striata. Forme jeune rappelant $R$. pilosa $\times 1.300$.

FIG. 33. M. hirsuta. Parasite jeune avec ses poils larges et son mucron antérieur $\times 1.300$.

FIG. 34. M. hirsuta. Parasite adulte; poils de la région postérieure $\times 1.300$.

FIG. 35. M. Elmassiani. Partie antérieure renflée avec le mucron hyaloplasmique $\times 1.300$.

FIG. 36. $M$. crenulata. Denticulations du péristome. Grains chromidiaux dans le cytoplasme $\times 1.300$.

Eia. 37. M. crenulata. Poils de la région postérieure. Région postérieure du cytoplasme dépourvue de grains de réserve $\times 1.300$.

FIG. 38. $M$, crenulata. Denticulations du péristome en voie de disparition $\times 1.300$.

FIG. 39. $M$. crenulata. Cytoplasme avec grains chromidiaux, noyau à nombreux karyosomes vacuolaires $\times 1.300$.

FiG. 40. Stomatophora coronata. Ventouse à collerette peu marquée et dont les stries se continuent sur l'ectoplasme $\times 650$.

Fi, 41. St. coronata. Noyau des grosses formes velues. Karyosome vacuolaire $\times 1.300$.

FIt. 42. St, coronata. Jeune parasite fusiforme, avec centrosome et différenciation canaliculaire ? dans le rostre. $\times 1300$.

FIG. 43. St. coronata. Jeune parasite ayant envahi un blastophore dénudé. Une vacuole claire avec un grain foncé accolé à sa paroi est visible dans l'extrémité arrondie de la Grégarine $\times 1.300$.

FIG. 44. St. coronata jeune. Centrosome au voisinage de la pointe, vacuole claire à l'autre extrémité. Sur la membrane nucléaire, un gros grain chromatique relié par un flament au karyosome $\times 1.300$.

Fia. 45. St. coronata. Ventouse pédonculée $\times 650$.

FIG. 46. St. coronata. Ventouse en vole de disparition; il n'en reste que des lambeaux $\times 650$.

FIG. 47. St. coronata. Ventouse en entonnoir à large ouverture. Début de l'extroversion $\times 650$.

FIG. 48. St. coronata. Ventouse extroversée en forme de plateau $\times 650$.

Fit. 49. St. coronata. Ventouse aspirant les spermatozoïdes flétris qui recouvrent le blastophore où s'est développé le parasite $\times 650$.

FIG. 50. St. coronata jeune. Ventouse; noyau à 2 karyosomes $\times 1.300$.

FIG. 51. St. coronata. Noyau avec karyosome coloré par la safranine $\times 1.300$.

FrG. 52. St. coronata. Noyau avec karyosome donnant des bourgeonnements arborescents $\times 1.300$.

FIG. 53. St. coronata. Karyosome vacuolaire avec un gros grain bourgeonné $\times 1.300$.

FIG. 54. St. coronata. Karyosome vacuolaire presque complètement décoloré avec un grain bourgeonné $\times 1.300$.

FIG. 55. St. coronata jeune, dans cavité intrablastophorienne. Dans l'extrémité arrondie une vacuole claire renferme un gros grain sidérophile relié par un flament à un épaississement de la membrane nucléaire $\times 1.300$.

FIG. 56. St coronata. Noyau normal; un grain accolé au karyosome $\times 1.300$.

FIG. 57. St. coronata. Un gros grain chromatique dans le noyau, à une certaine distance du karyosome $\times 1.300$

FIG. 58. St. coronata. Chromatine du noyau achromatique; karyosome en partie décoloré avec un grain fortement coloré accolé à sa surface $\times 1.300$.

FIG. 59. St. coronata. Chromatine bien colorée. Karyosome presque totalement décoloré; d'un croissant sidérophile placé à sa surface sort un fllament qui le relie à un gros grain chromatique. Un grain volumineux accolé au karyosome $\times 1.300$.

FIG. 60. St. coronata. Peu de chromatine dans le noyau. Karyosome incolore avec 2 grains colorés à sa surface $\times 1.300$.

FIG. 61. St. coronata. Karyosome incolore avec un grain coloré accolé à sa surface $\times 1.300$.

FIG. 62. St. coronata. Membrane nucléaire disparue ; plus de chromatine dans le noyau. Karyosome fortement coloré avec un grain bourgeonné $\times 1.300$. 
Fic. 63. St. coronata. Pas de membrane nucléaire. Pas de chromatine. Karyosome en grande partie incolore $\times 1.300$.

Fit. 64. St, coronata. Pas de membrane nucléaire. Karyosome presque incolore, pas de chromatine $\times 1.300$.

FIG. 65. St. coronata. Pas de membrane nucléaire. Pas de chromatine, pas de karyosome; à la place de celui-ci une plage où le suc nucléaire est un peu plus coloré $\times 1.300$.

FIG. 69. St. coronata. Parasite jeune sphérique avec nombreuses chromidies dans le cytoplasme $\times 1.300$.

Fid. 70. St. coronata. Parasite jeune fusiforme; noyau à karyosome double; à l'extrémité arrondie du parasite une zone de cytoplasme différencié.

Fra. 71. St. coronata jeune. Masso chromidiale s'échappant du noyau $\times 1.300$.

Fra. 72. St. coronata jeune dans cavité intrablastophorienne. Début de la ventouse $\times 1.300$.

Fig. 73. St. coronata. Deux parasites dans le même blastophore $\times 1.300$.

FIG. 66. St. diadema. Parasite avec lobes profonds $\times 650$.

FI\%. 67. St. diadema. Ornementation de l'épicyte bien visible, mais peu de lobes. Ventouse vue de face $\times 650$.

Fra, 68. St. diculeme. Parasite vu de profil $\times 650$.

\section{PLANCHE III}

Fia. 74. Monocystis agilis en dégénérescence. Hyperchromasie du suc nucléaire. Grains de paramylon peu nombreux et gonflés $\times 650$.

FIG. 75. M. agilis. Ratatinement des deux extrémités. Parasite en fuseau régulier. Noyau renfermé dans plage fortement colorée. Cytoplasme rempli de grains chromatoìdes auréolés $\times 650$.

Fia. 76. MI. lumbrici. Parasite hypertrophié. Surface creusée de cavités où sont logés des phagocytes $\times 650$.

FIG. 77. $\boldsymbol{M}$. lumbrici. Noyau d'un individu phagocyté. Un seul volumineux karyosome. Chromatine en grains très fins et très nombreux $\times 1.300$.

Fì. 78. Rhynchocystis pilosa. Extrémité antérieure. Chromatine dissoute dans le hyaloplasme de la trompe. Une zone plus colorée à l'emplacement du noyau $\times 1.300$.

FiG. 79. R. pilosa. Dégénérescence granuleuse $\times 1.300$.

FIa. 80. Monocystis lumbrici olidi. Noyau en pycnose. Dégénérescence granuleuse du cytoplasme. Plasmarrhexis $\times 1.300$.

FiG. 81-87. St. coronata en dégénérescence $\times 650$.

FIG. 81. Vacuolisation du eytoplasme. Hypertrophie du noyau.

FIG. 82-83. Dégénérescence granuleuse. Disparition de la chromatine.

FIG. 84-85. Ratatinement du noyau. Plasmarrhexis.

FIG. 86. Les grains de paramylon disparaissent. Apparition de vacuoles dans le cytoplasme. Noyau en mitose?

Fia. 87. Plus de grains de réserve. Vacuolisation du cytoplasme. Noyau en mitose ?

Fit. 88. Rhynchocystis pilosa. Métamérisation de la queue. Masses chromatoídes dans le cytoplasme $\times 1.300$.

FIG. 89. Monocystis agilis envahie par Bactéries. Noyau en dégénérescence. Masses chromatoïdes auréolées dans le cytoplasme $\times 1.300$.

FrG. 90. $\boldsymbol{M}$. striata envahie par Bactéries $\times 1.300$.

FIG. 91, 92, 93. St. coronata attaquées par Bactéries $\times 1.300$.

FIG. 93, 91. Début de l'envahissement.

FIG. 92. Microbes condensés au centre du parasite en une sphère compacte, sorte de sclérote. A côté le noyau de la Grégarine est encore visible.

FiG, 94. Rhynchocystis pilosa.remplie de Bactéries. Région antérieure avec noyau en dégénéres. cence $\times 1.300$.

FIG. 95. Monocystis lumbrici attaquée par Bactéries. Grains de paramylon gonflés, en voie de disparition. Disparition de la membrane nucléaire. Pycnose du noyau $\times 1.300$.

Fic. 96. M. striata. Parasite fortement tordu; côtes de sarcocytes se fragmentant en boules qui se détachent. 
FIG. 97. Fragment d'une coupe de pavillon séminal cilié chez Lumbricus herculeus. Cellules phagocytaires en saillies; entre elles des paquets de spermatozoüdes sont entassés parallèlement, la tête piquée entre les cils $\times 750$.

FIt. 98. Nematocystis magna. Jeune parasite renfermé dans une cellule épithéliale; celle-ci est énormément hypertrophiée et montre déjà en son centre las racines ciliaires très accentuées, se prolongeant sous l'épithélium $\times 1.300$.

\section{PLANCHE IV}

Toutes les figures de cette planche représentent des coupes colorées par l'hématoxyline et le Lichtgrün, l'éosine ou l'orange $\times 750$.

Fra. 99. Deux Pleurocystis Cuenoti. Région antérieure adhérente à des cellules géantes de l'épithélium des pavillons séminaux de $\boldsymbol{H}$. longus; celles-ci font saillie hors de l'épithélium. Dans leur intérieur, racines ciliaires développées en fortes tono-flbrilles par la traction du parasite.

Frg. 100. Epithélium des pavillons séminaux de $L$. herculeus. Une cellule phagocytaire très développée fait saillie à la surface, elle renferme des spermatozolides englobés. Dans l'épaisseur de l'épithélium, une autre cellule bourrée de granulations jaunâtres résidus de digestion.

FrG. 101. Extrémité antérieure de Nematocystis magna enserrée dans une cellule cupuliforme. Les côtes ectoplasmiques du parasite s'enfoncent dans la cellule-hôte et s'entremêlent aux tonofibrilles de celles-ci qui se prolongent fort loin dans l'épithélium. Le noyau de la cellule cupuliforme est en dehors de la coupe.

Fig. 102. Cellule cupuliforme en dégénérescence. Une partie seulement đu centre vacuolaire de cette cellule est reprêsentée.

FIG. 103. Cellule cupuliforme. Début de la dégénérescence vacuolaire. La coupe ne passant pas tout à fait par l'axe de la cellule, le trone fibrillaire axial n'est pas visible.

Fia. 104: Fragment d'une coupe des canaux déférents. Épithélium cylindrique cilié. Cellules aux racines ciliaires très développées se prolongeant sous l'epithélium en un feutrage qui constitue la basale.

FIG. 105. Noyau et karyosome de $N$. magna. Striations de la cuticule visibles à gauche de la figure.

FIG. 106. Une cellule saillante de l'épithélium des pavillons séminaux présentant les caractères de la cellule cupuliforme mais ne renfermant pas de Grégarine.

\section{PLANCHE V}

F16. 107. Jeune Monocystis agilis au centre d'une spermatosphère de L.herculeus. Début de l'attraction des noyaux des spermatogonies. Idiozomes presque tous dans la région distale $\times 1.300$.

FIG. 108. Jeune $\boldsymbol{M}$. agilis. Noyau avec karyosome entouré d'un cercle de grains chromatiques. Cytoplasme des spermatogonies accumulé dans leur région distale et devenant vacuolaire; il renferme l'idiozome $\times 1.300$.

FIG. 109. Jeune $\boldsymbol{M}$. agilis au centre d'une spermatosphère. Spermatocytes $I$ avec noyaux en spirème $\times 1.300$.

FIG. 110. Jeune $M$. agilis dans blastophore, elle renferme une vacuole à l'un de ses pôles. Etirement en lanière du cytoplasme des cellules sexuelles $\times 1.300$.

FIG. 111. Jeune $M$. agilis dans blastophore; elle renferme 2 volumineuses masses chromidales dans son cytoplasme. L'étirement du cytoplasme des spermatocytes s'accentue $\times 1.300$.

FiG. 112. Jeune $M$. agilis dans spermatosphère. Noyau du parasite hypertrophié. Cytoplasme des spermatogonies réfugié dans la région distale et renfermant l'idiozome $\times 1.300$.

FIG. 113. Spermatogonies normales de $L$. herculeus $\times 1300$.

FiG. 114 a 131. Divers stades de la spermatogénèse normale et de la spermatogénèse pathologique de $L$. herculeus $\times 1.300$. 
Hia. 114. Début de la dégénérescence du noyau dans les spermatocytes supportés par un blastophore parasité. Etirement et vacuolisation du cytoplasme.

FTG. 116. Dégénérescence des noyaux continuant. Réseau hyperchromatique commençant à se fragmenter. Accumulation de la chromatine sur la.membrane. Cytoplasme étiré en une mince lanière.

FIG. 117-118. La chromatine se tasse pendant que le noyau s'étire.

FIG. 131. Etirement irrégulier. Chromatine en grains fins non encore soudés.

FIG. 120, 121, 122. Condensation et étirement de la chromatine, diminution du cytoplasme.

FIG. 127-128. Spermatocytes I et Spermatocytes II normaux.

Fig. 123. Spermatides normales.

FiG. 124. Une Grégarine dans le blastophore. Le parasite n'est pas représenté. Chromatine sur la membrane des cellules sexuelles. Cytoplasme centrifuge. Idiozo-centrosome a la périphérie. Sortie du fllament caudal. Fixation au Flemming.

FIG. 125. Stade semblable au précédent. Noyau plus petit, cytoplasme plus étiré. Centrosome dédoublé. Grain proximal sur la membrane nucléaire, relié au flament par une traînée de granulations. Fixation au Flemming.

Fia. 126. Suite du précédent. Filament cytoplasmique grêle et allongé. A la base de la partie conique on voit un grain très coloré rattaché à la membrane nucléaire par un grêle filament. Fixation au Flemming.

FIG. 115. Stade semblable au précédent, mais filament cytoplasmique irrégulier et centrosome non visible.

FIG. 129. Spermatides normales en voie d'étirement. Aussenkörper.

FIG. 130. Spermatozoîdes normaux.

FIG. 132. Rhynchocystis pilosa jeune dans blastophore. Mrucron déja visible, cytoplasme chargé de grains chromidiaux. La chromatine commence à se condenser dans les cellules sexuelles. Aussenkörper grêle. $\times 1.300$.

FIG. 133. $R$. pilosa dans un blastophore. Le mucron est entouré de crêtes saillantes supportant des poils. Cytoplasme chargé de chromidies. Chromatine déjà très condensée dans les cellules sexuelles. $\times 1.300$.

FiG. 134. $R$. pilosa à tête piqué dans un blastophore. Condensation de la chromatíne dans les cellules sexuelles et étirement du cytoplasme en flament conique court. $\times 1.300$.

Fit. 135. $R$. pilosa dans blastophore. Condensation de la chromatine dans les cellules sexuelles. Le fllament caudal et l'aussenkörper sont visibles. $\times 1.300$.

\section{PLANCHE VI}

FIG. 136. Jeune Stomatophora coronata dans blastophore $\times 650$.

Frg. 137. Jeune St. coronata pénétrant dans un blastophore qui porte des spermatocytes II. Zone différenciée à l'extrémité postérieure du parasite. Un grain très coloré sur la membrane nucléaire. Cytoplasme des cellules sexuelles accumulé dans la région distale $\times 1.300$.

FrG. 138. Jeune St. coronata venant de pénétrer dans le pédicule d'un spermatocyte I. Canal clair dans le rostre. A côtẻ grain centrosomien relié à la membrane nucléaire. Un grain très coloré placé sur la membrane est relié au karyosome $\times 1.300$.

FIG. 139. St. coronata emplissant un blastophore recouvert de spermatozoĩdes flétris aciculaires $\times 650$.

FIG. 140. St. coronata emplissant un blastophore qui supporte des cellules sexuelles altérées. La chromatine est condensée dans celles-ci en un bloc en forme de larme batavique $\times 650$.

FIG. 141. St. coronata dans blastophore. Les cornichons chromatiques, seuls restes des cellules sexuelles, quittent leur position radiale et se rapprochent de la ventouse $\times 650$.

Fra. 142. St. coronata dans blastophore. Tous les cornichons chromatiques sont rassemblés en face de la ventouse. La membrane blastophorienne enveloppant la Grégarine est visible $\times 650$.

Frg. 143. St. coronata. Coupe d'une Grégarine à toison appendue par sa rentouse à la paroi de la cavité qu'elle s'est creuséc dans un blastophore. Cellules sexuelles à cytoplasme étiré en lanière piriforme, noyau réduit à la membrane et à quelques grains chromatiques, $\times 650$. 
IIG. 144. St. coronata. Jeune Grégarine à toison dans blastophore. Karyosome entouré par cercle de gros grains chromatiques. Accolée au noyau une zone cytoplasmique différenciée renfermant une masse chromidiale. Cytoplasme des cellules sexuelies étiré en alène $\times 1.300$.

FrG. 145. St. coronata. Grégarine velue avec sa toison de cellules sexuelles altérées $\times 650$.

FIG. 146. St. coronata. Cellules sexuelles altérées formant le revêtement des Grégarines à toison $\times 1.300$.

FIG. 147. St. coronata dans blastophore. La chromatine des cellules sexuelles est attirée dans le blastophore $\times 1.300$.

FIG. 148. Spermatogonie normale de Pheretima $\times 1.300$.

FIG. 149. St. coronata dans blastophore. Cellules sexuelles longuement étirées et couchées sur le blastophore. Chromatine tassée $\times 1.300$.

FIG. 150. St. coronata dans blastophore. Altération des cellules sexuelles, Chromatine moins compacte que dans le cas précédent. Au centre de la lanière cytoplasmique, on voit le filament axile s'échappant du centrosome placé sur la membrane nucléaire × 1.300

FIG. 151. Spermatocytes II, normaux $\times 1.300$.

FIG. 152. St, coronata dans blastophore. Etirement du cytoplasme des Spermatocytes II, début du tassement de la chromatine $\times \mathbf{1 . 3 0 0 .}$

F19. 160. St. coronata dans blastophore. Cytoplasme très étiré, filament caudal s'échappant do l'extrémité. Un grain très coloré à sa base. Chromatine fortement tassée $\times \mathbf{1 . 3 0 0}$.

FIG. 161. St. coronate dans blastophore. Chromatine en bâtonnet court, cytoplasme en lanière longue et étroite, conique $\times 1.300$.

Fig. 153, St. coronata dans blastophore. Début de la concentration de la chromatine dans les cellules sexuelles. Cône cytoplasmique différencié unissant les grains centrosomiens proximaux sur la membrane nucléaire au grain distal placé à la base du filament axile $\times 1.300$

FIG. 154. St. coronata dans blastophore. Tassement de la chromatine, diminution de volume du cytoplasme $\times 1.300$.

FrG. 162-163-164. St. coronata dans blastophore. Chromatine compacte en un cornichon court de forme variée $\times 1.300$.

FIG. 155: Spermatides normales $\times 1.300$.

FrG. 156. Kyste de St, coronata recouvert par blastophores portant leurs cellules sexuelles aitérées $\times 1.300$.

FIG. 157. St. coronata dans blastophore. Cytoplasme très étiré. Chromatine commençant à se tasser $\times 1.300$.

Fig. 158-159. St. coronata dans blastophore. Chromatine en bâtonnet compact. Cytoplasme en filament étroit $\times 1.300$.

Fic. 165. Spermatide normale en voie d'étirement $\times 1.300$.

FIG. 166. St. coronata dans blastophore. Spermatides montrant le cytoplasme étiré en lanière conique indépendante du filament caudal $\times 1.300$.

Fra. 167. Spermatozoïdes normaux. Le début seul de la queue est représenté $\times 1.300$.

Fic. 168-169-170. St. coronata dans blastophore. Spermatozoïdes altérés $\times 1.300$.

\section{PLANCHE VII}

Fixation au Bouin-Duboscq. Coloration au Mann.

FIG. 171. Jeune Monocystis agilis. Masses chromidiales dans le cytoplasme $\times \mathbf{1 . 3 0 0}$.

FIG. 172. Jeune $\boldsymbol{M}$. agilis. 3 masses chromatiques accolées au karyosome. Masses chromidiales dans le cytoplasme $\times 1.300$.

EIG. 173. M. agilis. Chromatine accumulée autour du karyosome, réseau non visible $\times 1.300$.

FıG. 174. M. agilis. Le karyosome tangent à la membrane nucléaire est formé de 2 régions, l'une de plastine, l'autre de chromatine; celle-ci fait hernie dans le cytoplasme $\times 1.300$.

EIG. 175. M. agilis. Noyau sans memorane, karyosome à contours peu tranchés, sue nucléaire très coloré $\times 1.300$.

Ere. 176. $M$. agilis. Noyau renfermant un grain probablement bourgeonné par le karyosome. Chromatine en réseau $\times 1.300$. 
FIG. 177. Pleurocystis Cuenoti. $\times 1.300$. Noyau avec karyosome arborescent, formé de 2 parties : chromatine entourée par plastine ramifiée.

FIc. 178. P. Cuenoti. Karyosome simple $\times 1.300$.

Fig. 179. P. Cuenoti. Karyosome semblable à celui de la figure 177 mais à plastine non ramiflée $\times 1.300$.

FIG. 180. Noyau normal de $M$. lumbrici $\times 1.300$.

FIG. 181. Monocystis lumbrici phagocytée. Karyosome moniliforme. Au centre plastine en rouge, autour zone chromatique $\times 1.300$.

FI. 182. Nematocystis magna coupe de la cellule cupuliforme renfermant le parasite. Cotes du parasite engrénées dans les stries du plateau de la cellule $\times 750$.

Fir. 183. Monocystis lumbrici olidi. Noyau montrant les 2 karyosomes tous deux vacuolaires $\times 1.300$.

F1' 184-185. M. Le Mêmei. Noyau avec les deux karyosomes, 2 stades différents $\times 1.300$.

FIG. 186. Zygocystis pilosa. Noyau avec le karyosome très volumineux. Poils larges $\times 1.300$.

FIG. 187 à 193. Stomatophora coronata $\times 1.300$.

Fra. 187. Karyosome formé de 2 parties accolées. Cytoplasme bleu. Les spermatides moniliformes sont très diversement colorées.

Fir. 188. Cytoplasme violet renfermant gouttelettes rouges. Suc nucléaire violet.

Fig. 189. Karyosome en eroissant ; membrane nucléaire disparue en un point.

FIG. 190. Karyosome violet. Gouttelettes rouges dans le cytoplasme.

FIG, 191. Noyau normal; karyosome vacuolaire.

Fie. 192. Parasite jeune montrant le début de la ventouse.

FIG. 193. Parasite jeune, amas de grains rouges entre la ventouse et le noyau. 



\title{
SECONDE THESE
}

\section{PROPOSITIONS DONNÉES PAR LA FACULTÉ}
Géologie. - Variations de faciès des terrains crétacés dans le Dauphiné.

Botanique. - Les Myxomycètes.

\author{
Vu et approuvé. \\ Paris, le 22 Juillet 1909. \\ LE DOYEN DE LA FACULTÉ DES SCIENCES, \\ Paul APPELL
}

Vu et permis d'imprimer :

LE VICE-RECTEUR DE L'ACADÉMIE DE PARIS,

L. LIARD 


Arch de Zool. Exple et Gén.
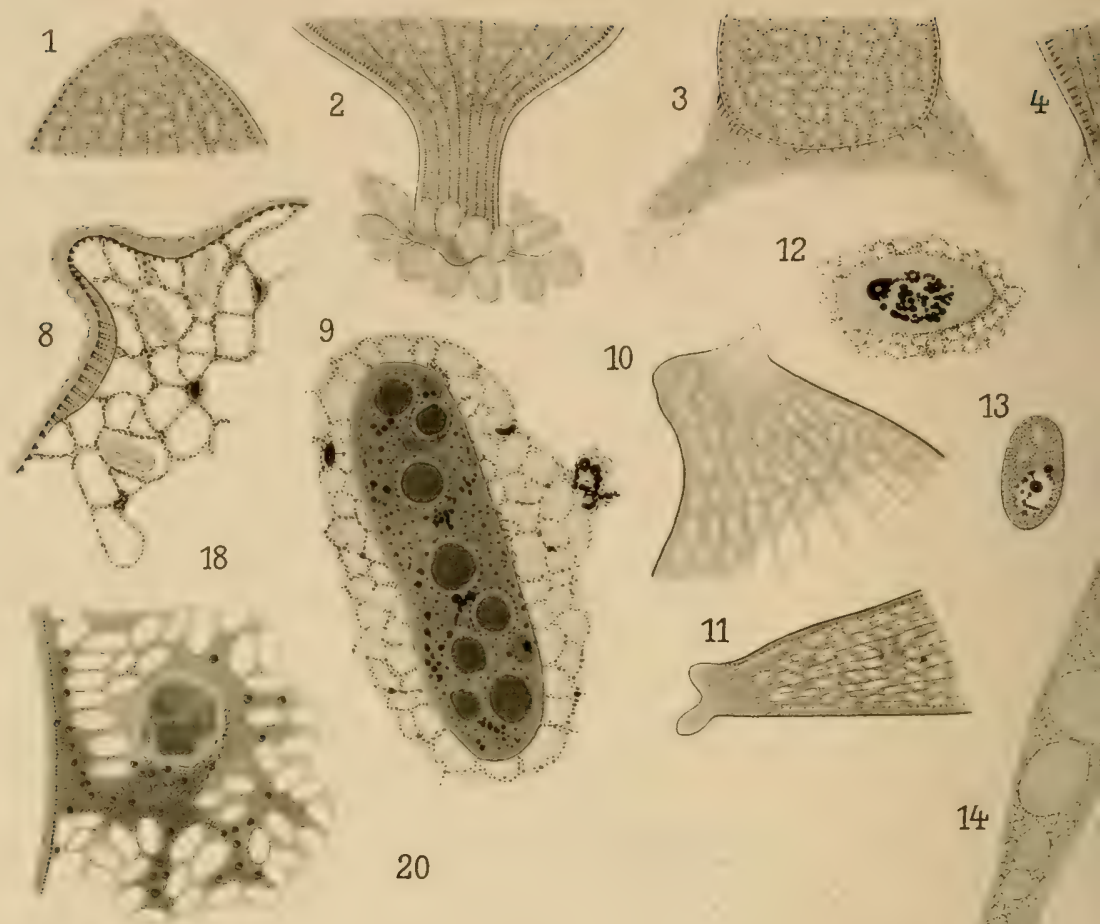

20

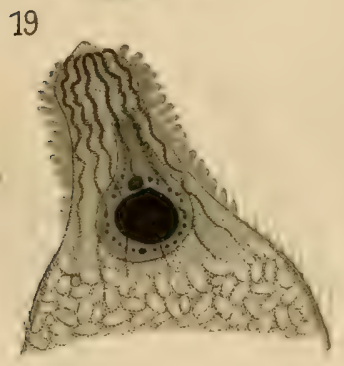

24
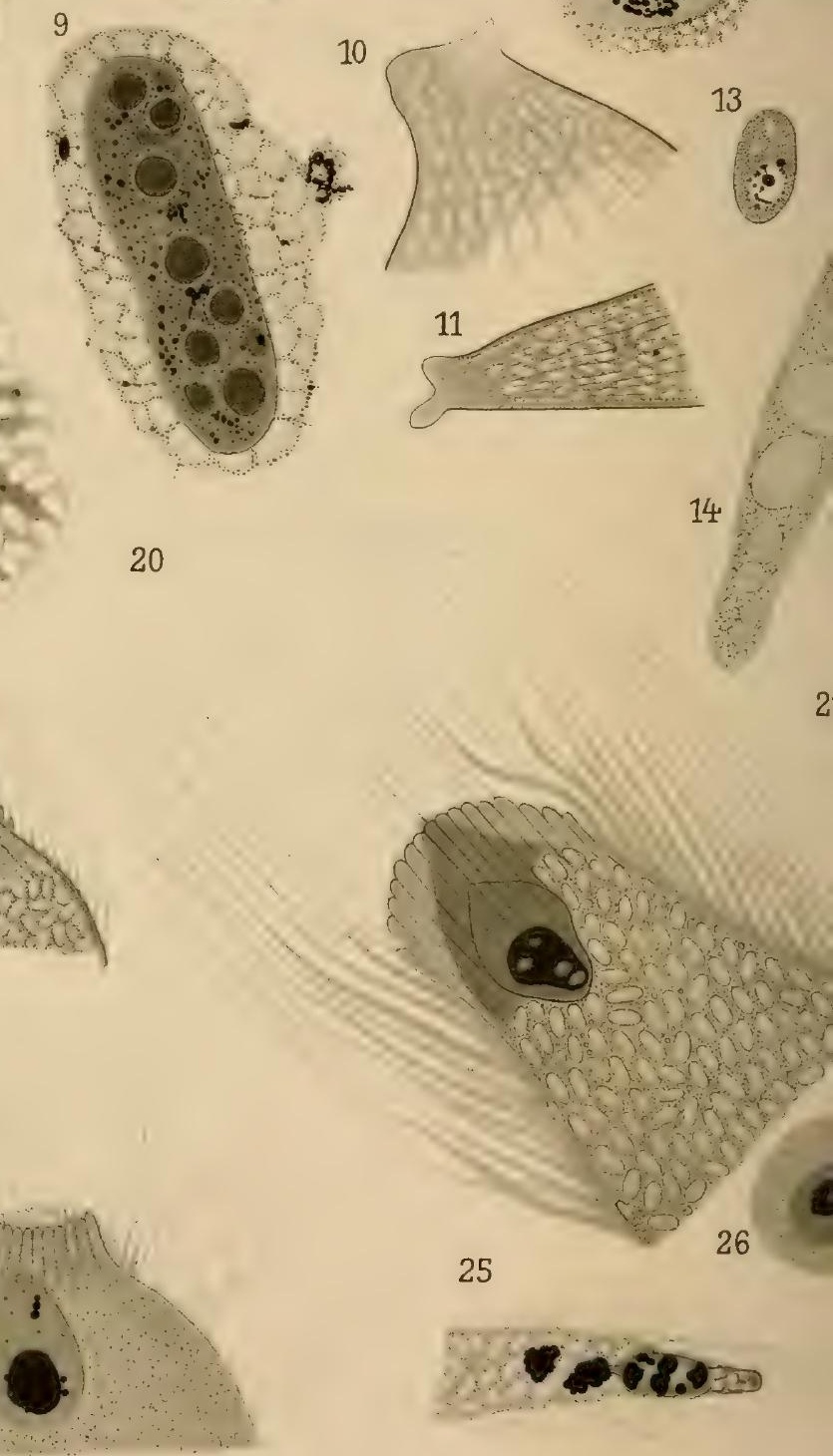

Arch de Zool. Expg. et Gén?

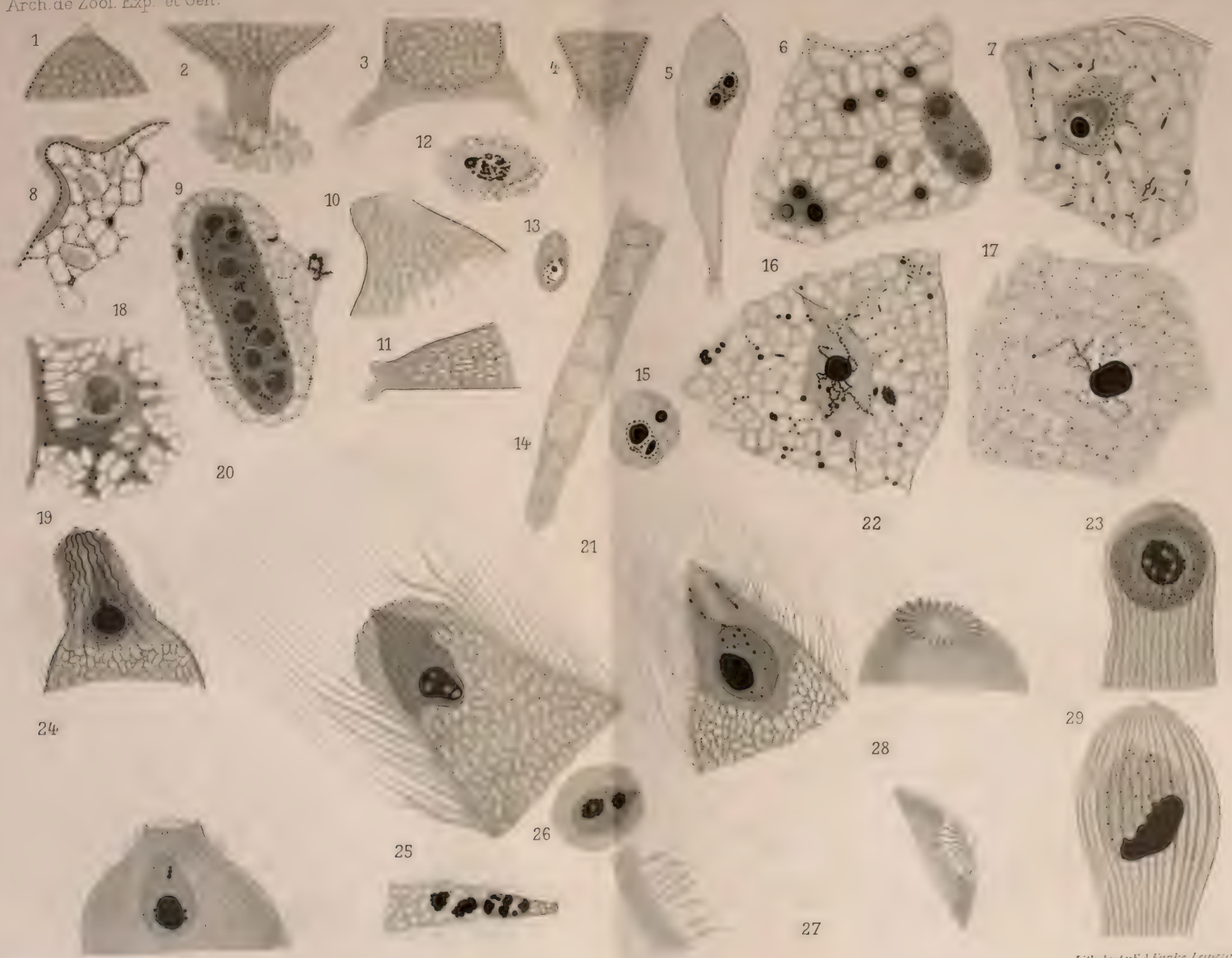




Arch de Zool. Exp. le et Gén?

30
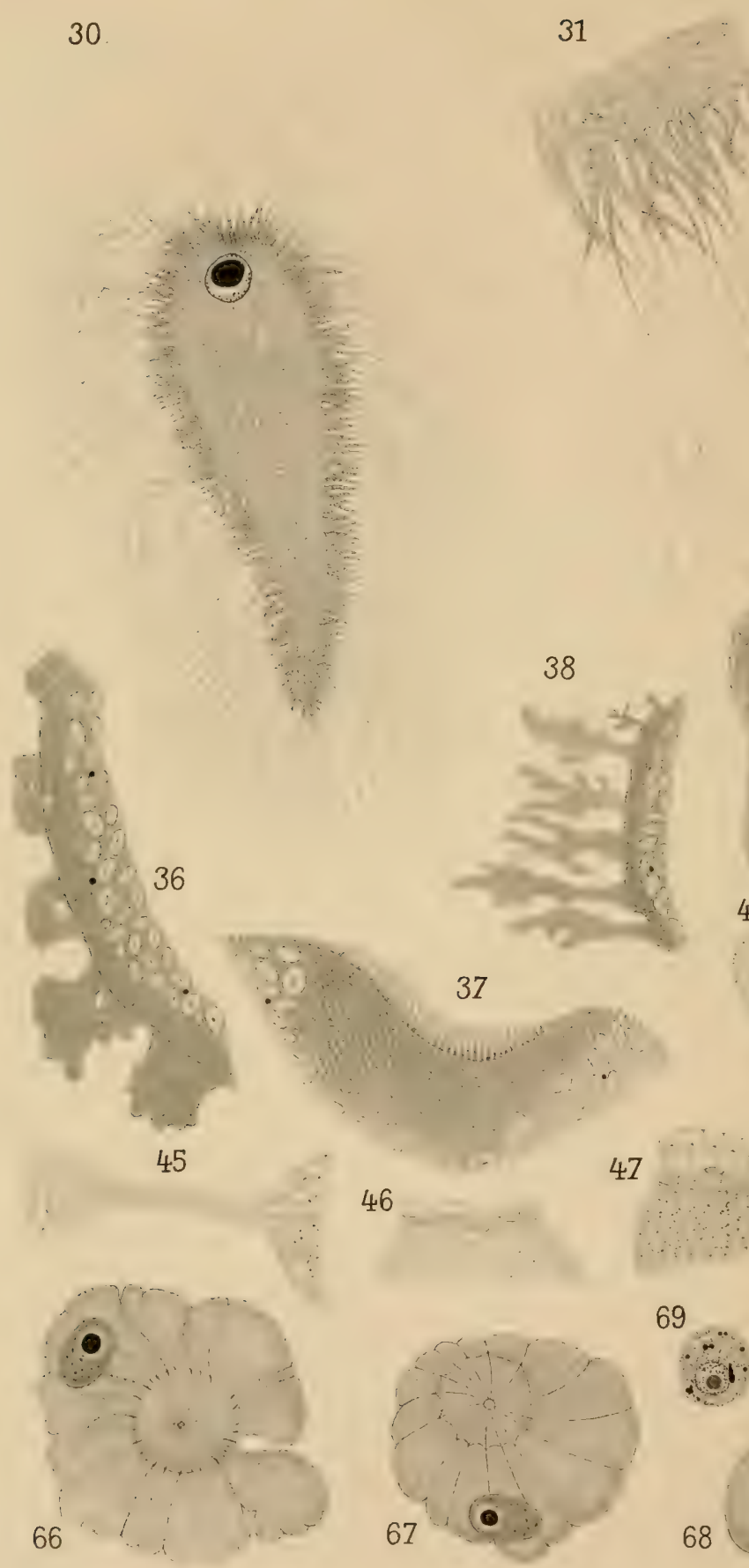

38

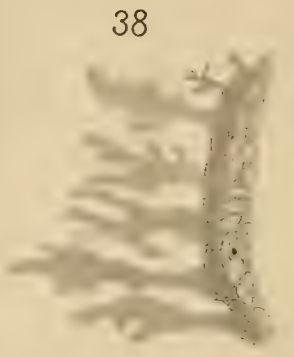

37

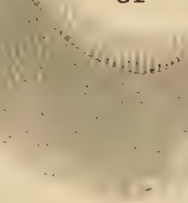

46

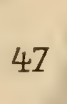

\section{8}
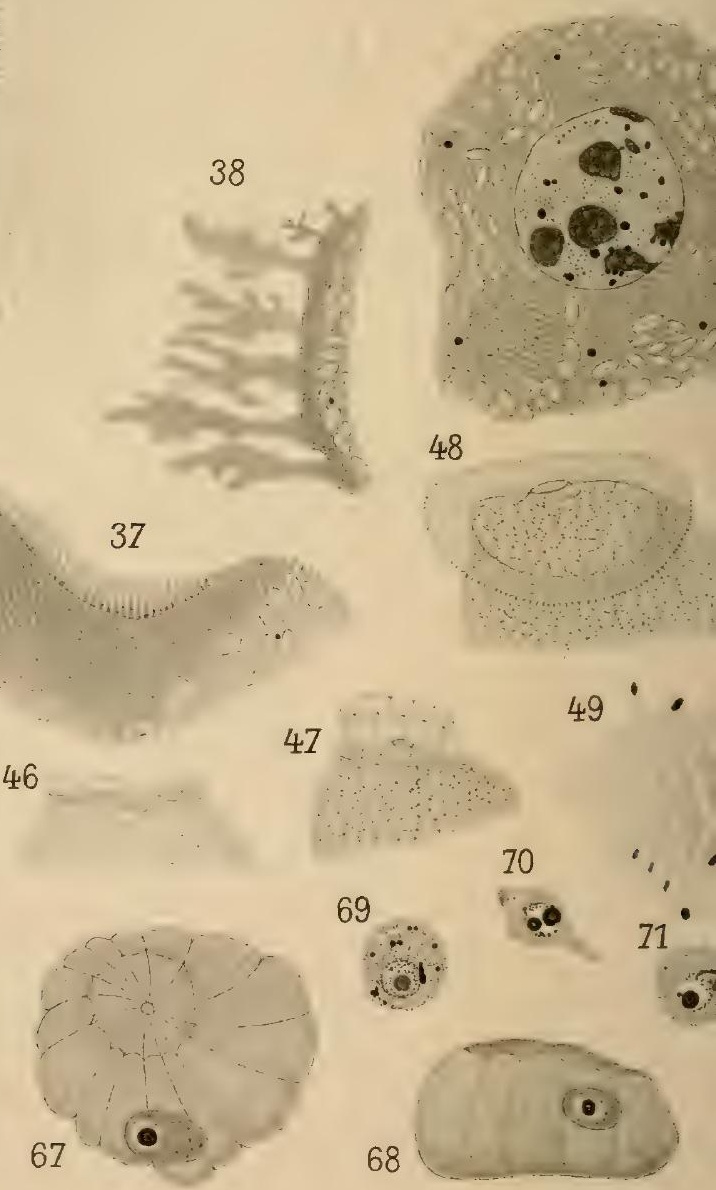

Ed. Hesse del.

RHYNCHOCYSTIS PILOSA, MONOCYSTIS STRTATA, M.HIRSUTA, 


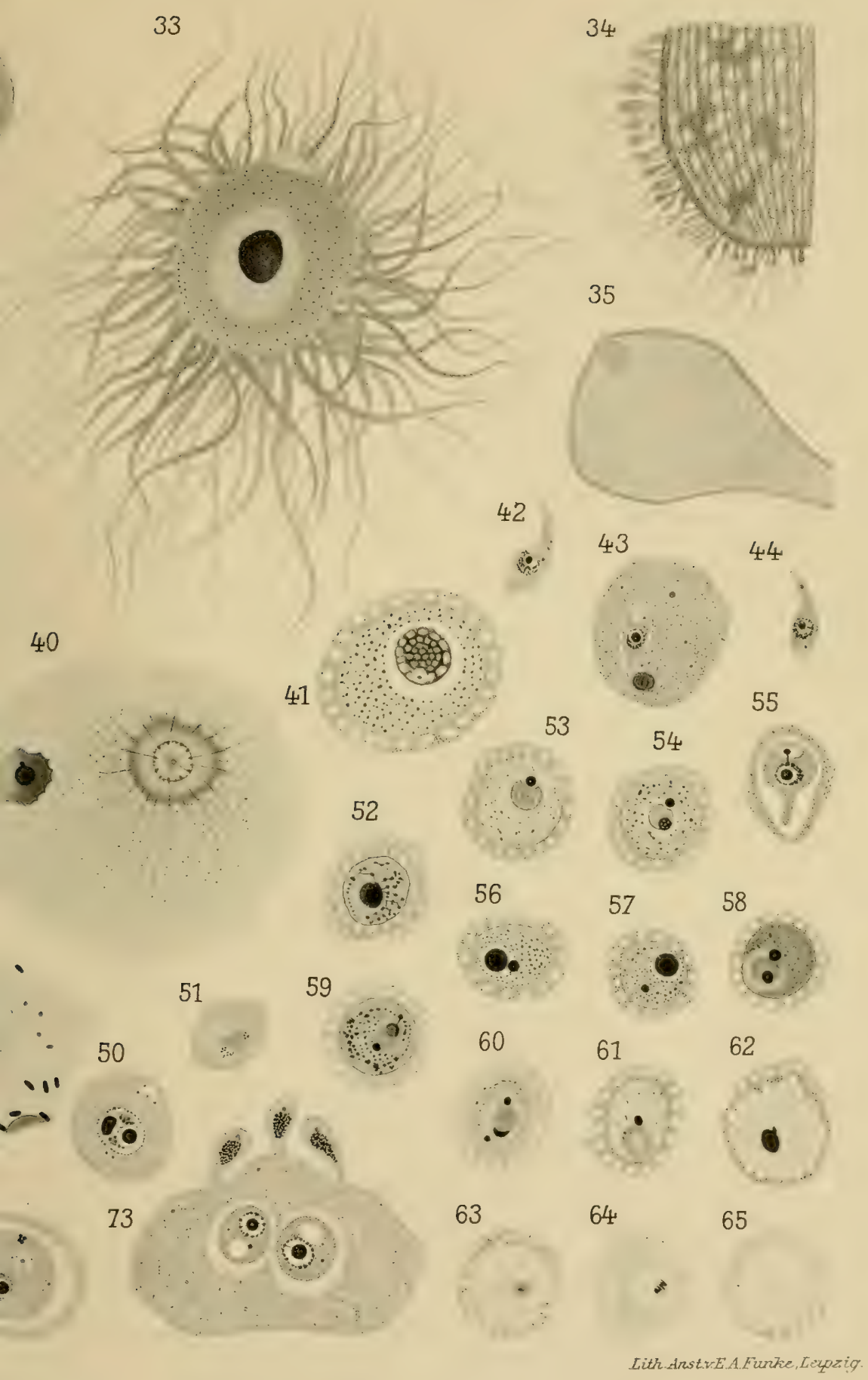

ENUIATA, M. ELMASSIANI, STOMATOPHORA DIADEMA, S.CORONATA 

Arch de Zool. Exp. et Gén?

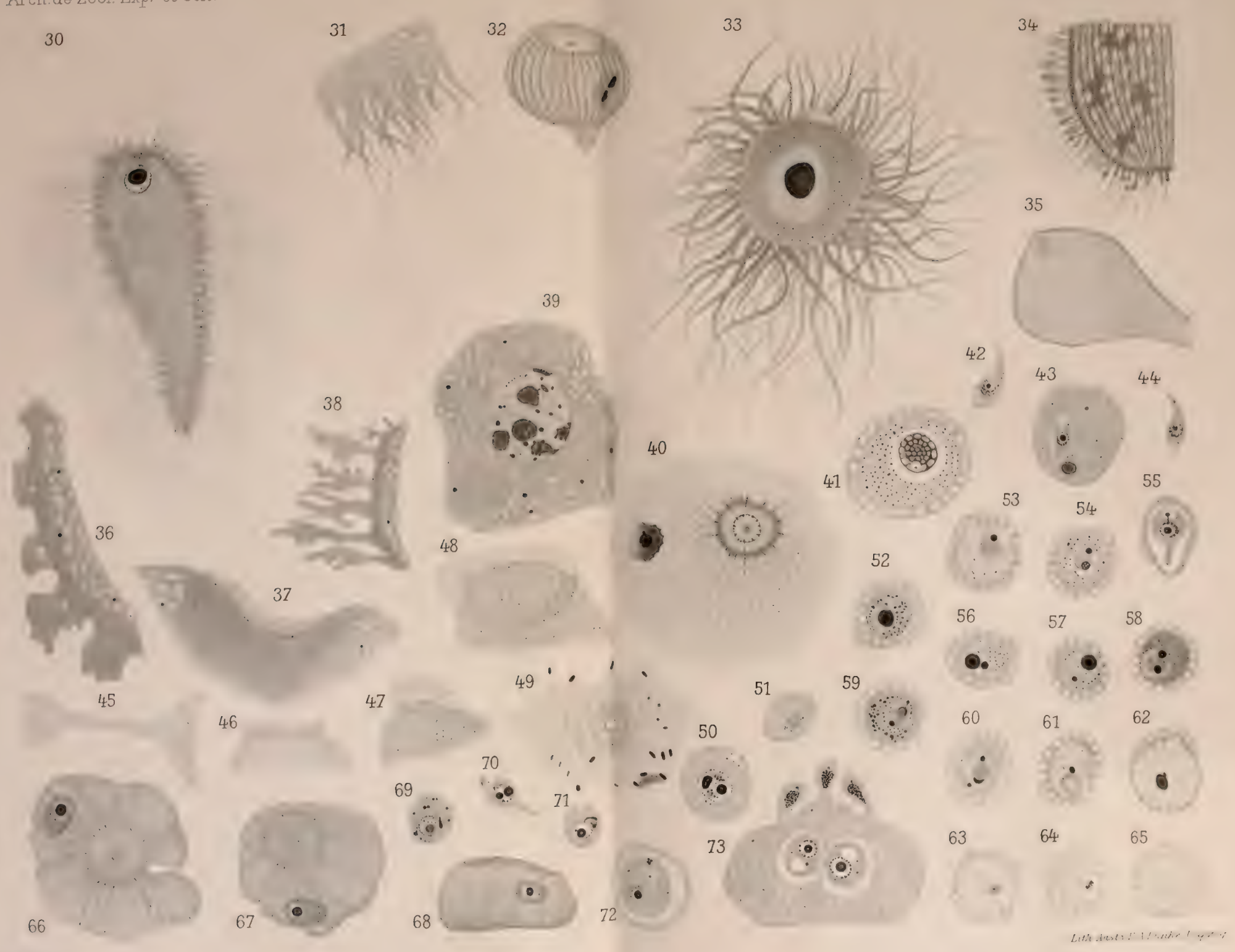

Edullesse del

RHYNCHOCYSTIS PIIOSA NONOCYSTIS STRTATA M HIRSUTA N CRENULATA, M. ELMASSIANI, 


Arch de Zool. Expp. et Gén.
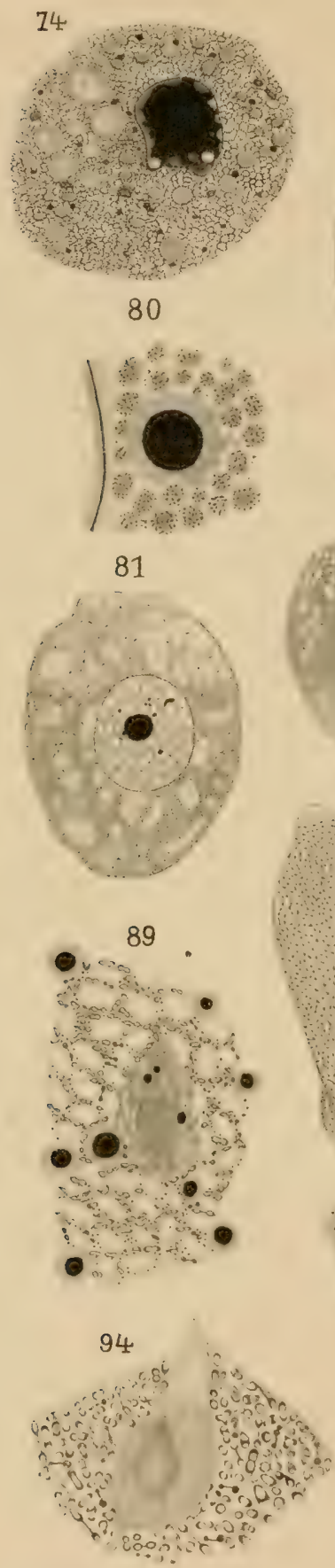

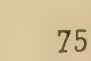

75

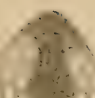

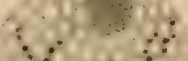

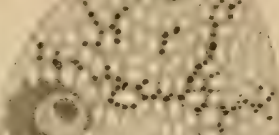

$+1, \ldots+\ldots \therefore \cdots$

wate?

$\therefore$ is:

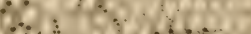

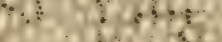

on.
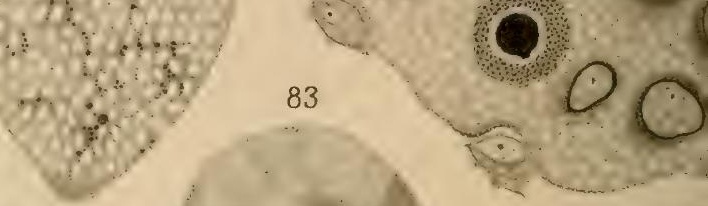

82
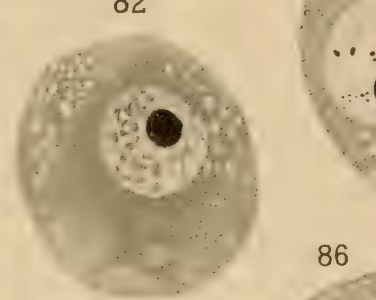

0

20

86

90
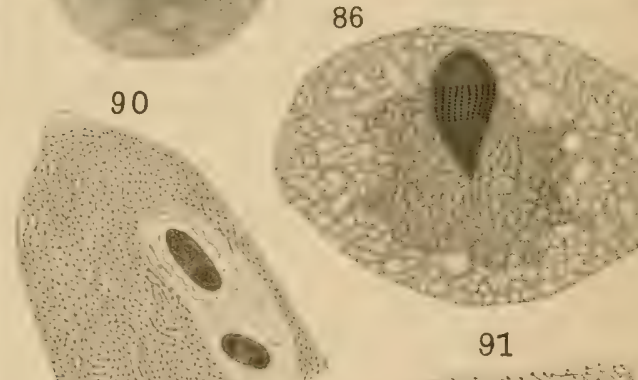

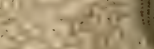

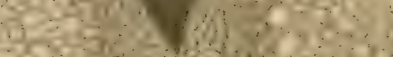

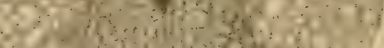
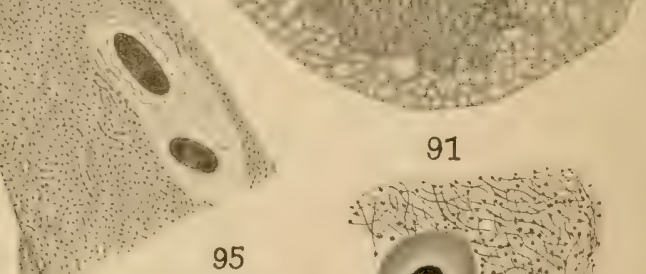

95
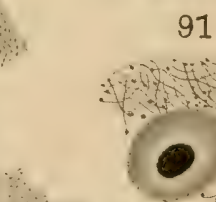

91

84

50

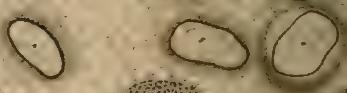

Wiste:
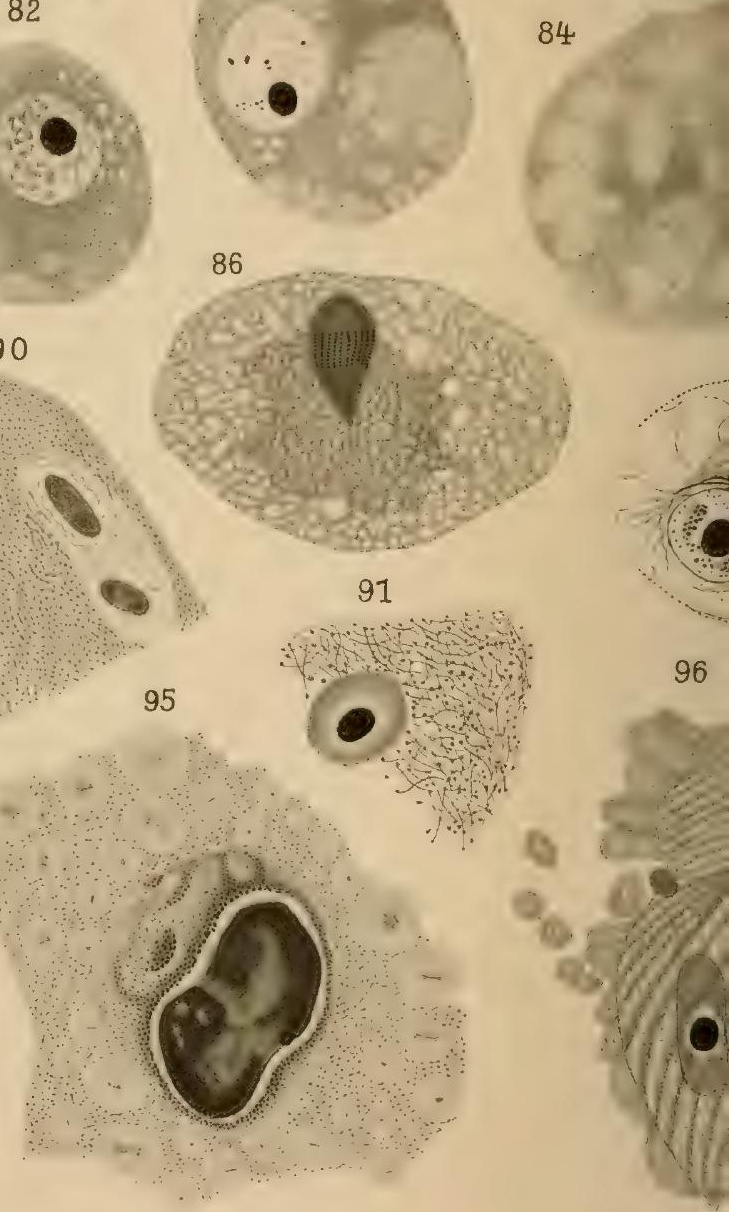



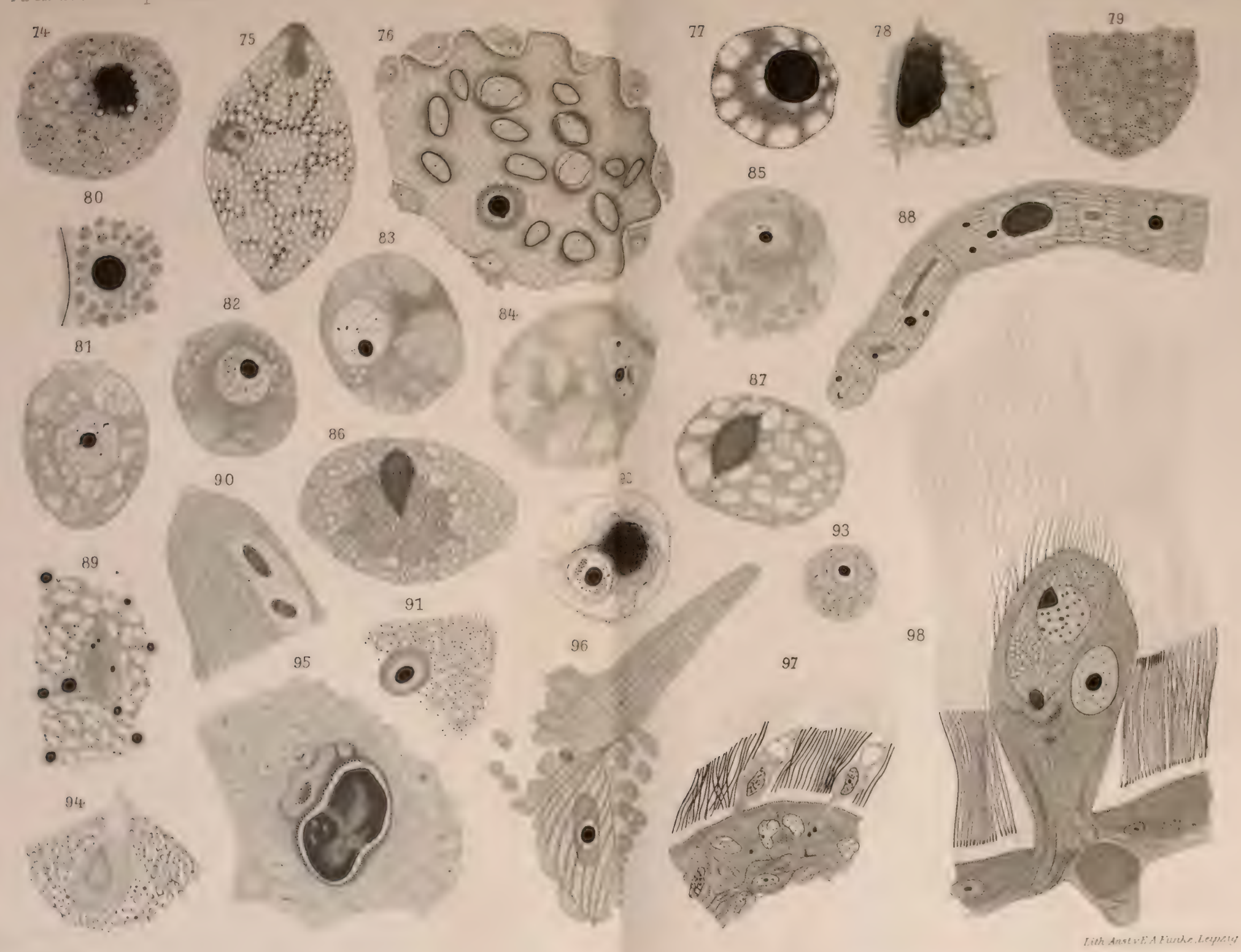

Rii. Hesce del 


Arch de Zool. Exp. le et Gén!

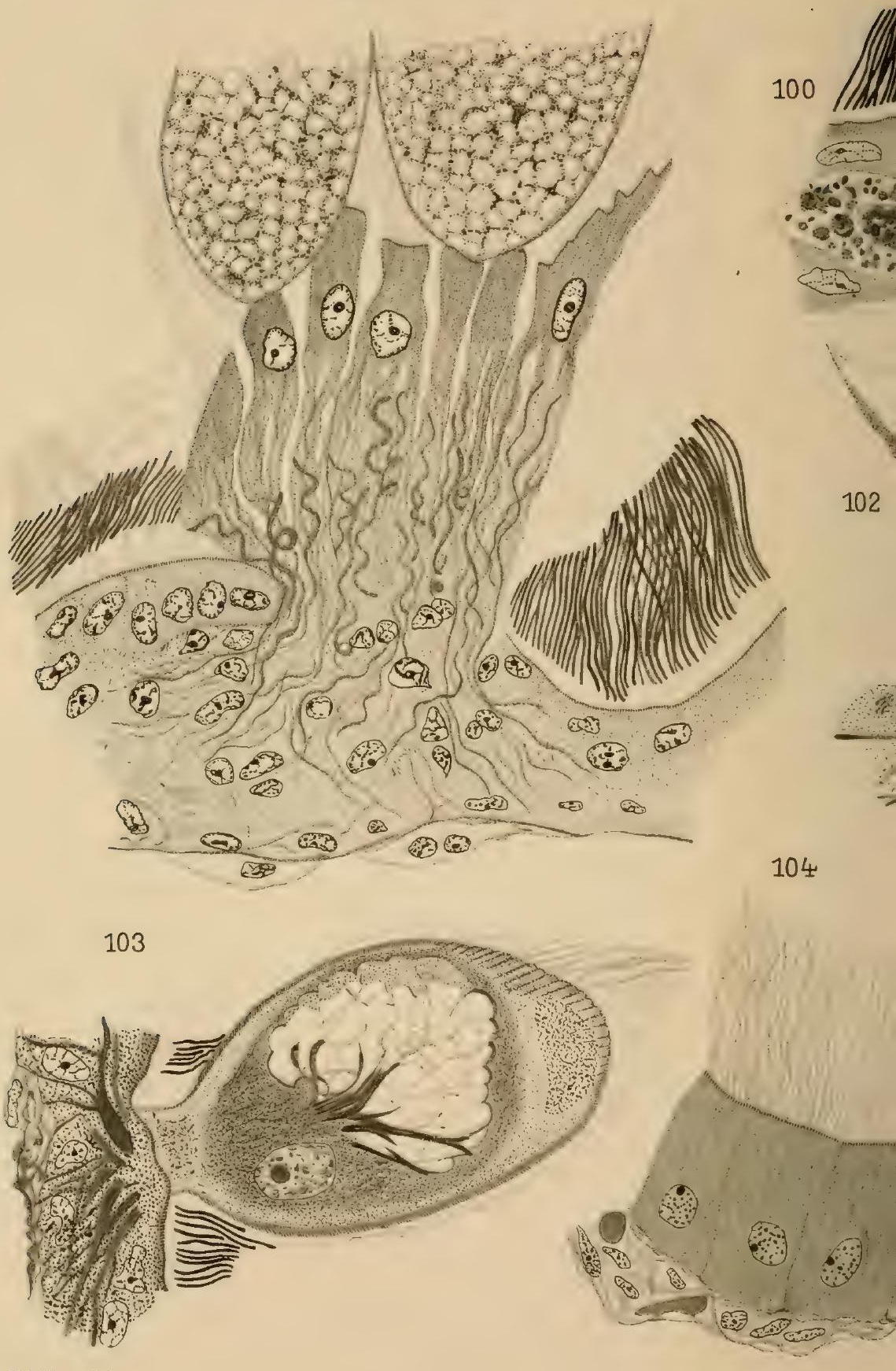

Ed. Hesse del. 


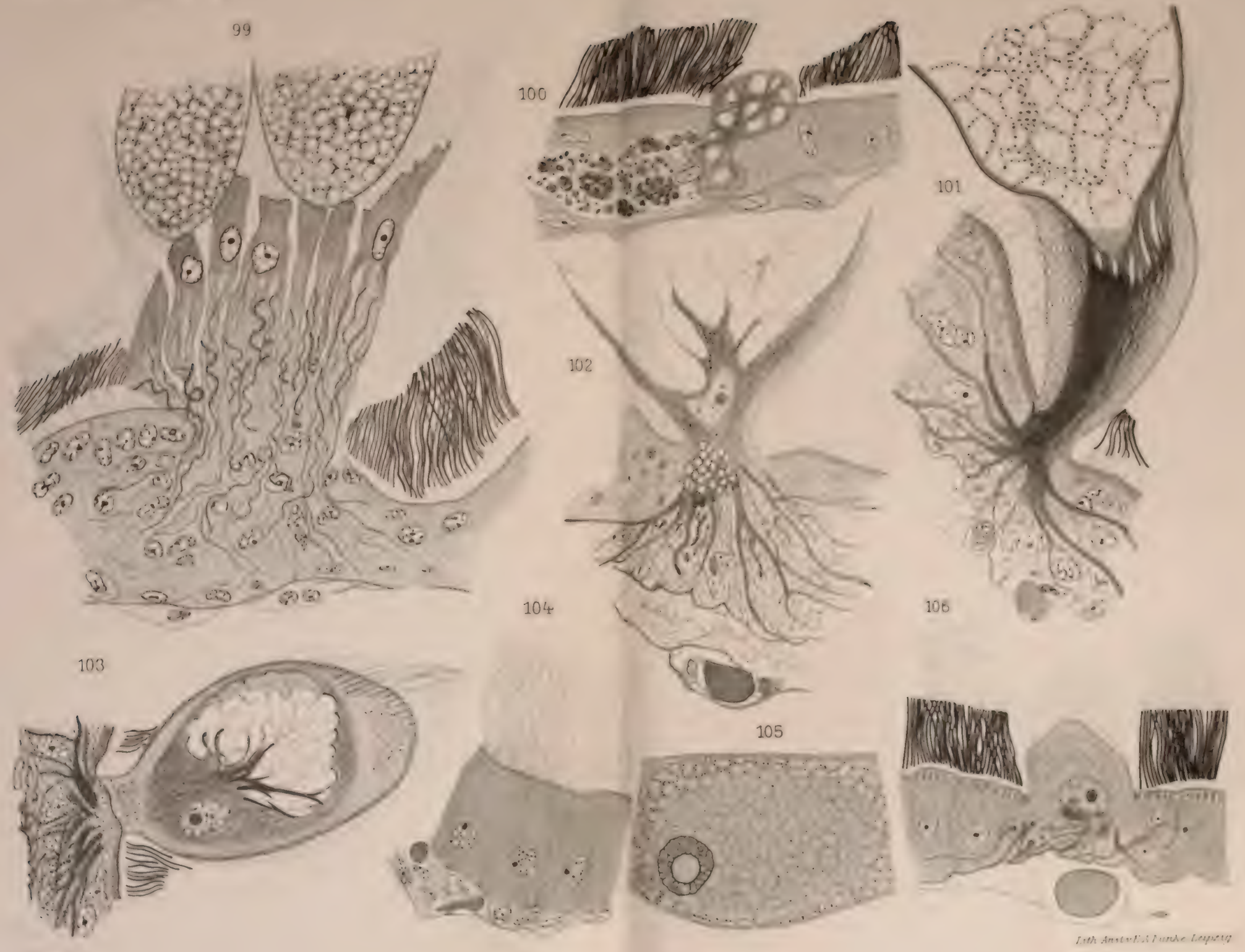





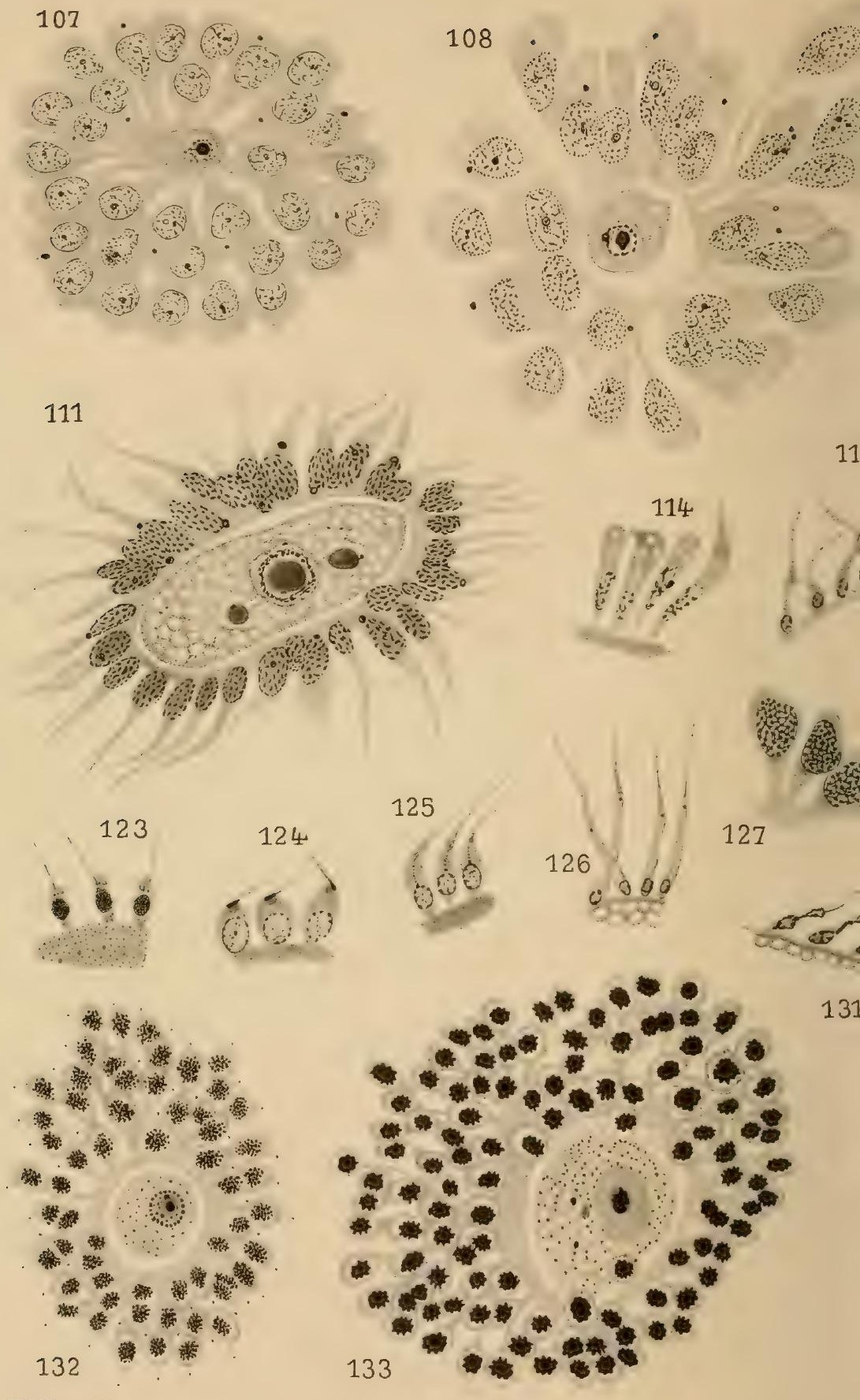

Ed.Hesse del 
109

को का का की

8

क क 6

b 6

as:

(6)

a

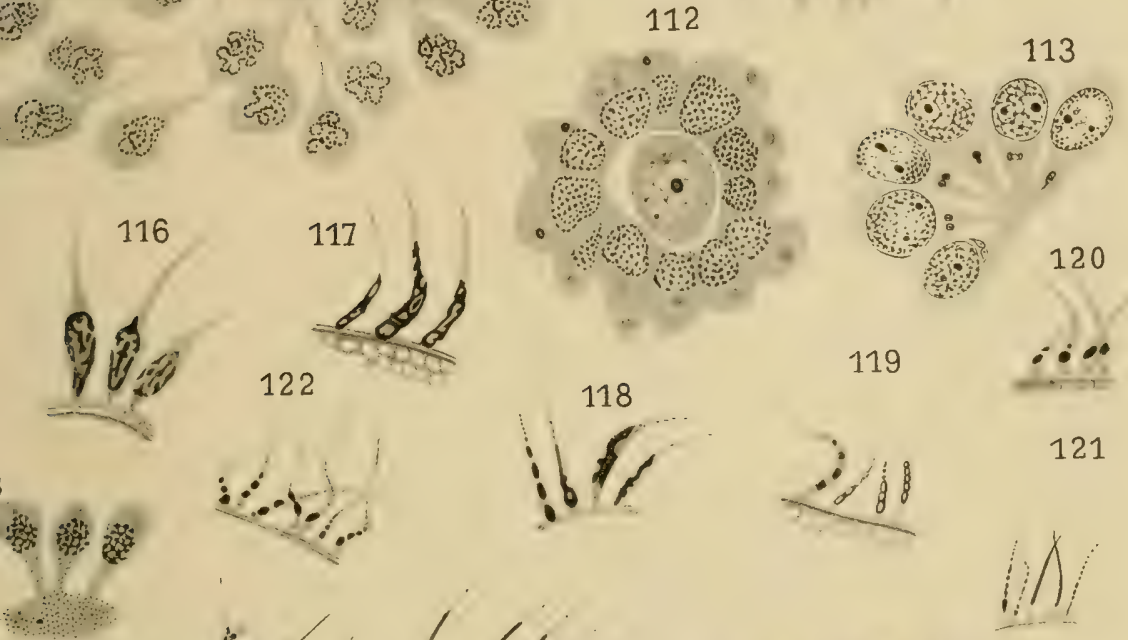

110

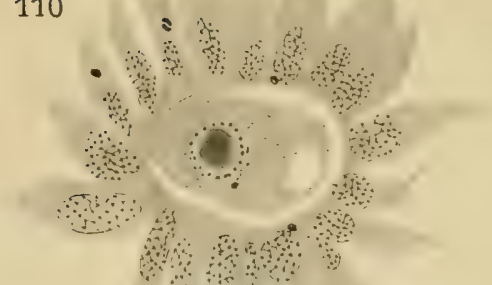


Arch do Zool. Exis? ot Gón?

111

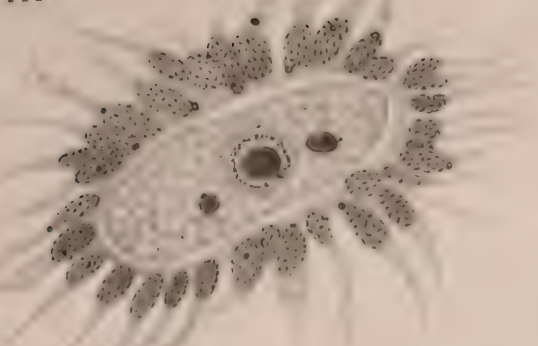

\section{ใ:}

c.

$\therefore \quad$
$\therefore$

\section{$109 \ldots 8$}

$80 \quad \therefore \therefore \div$

ches

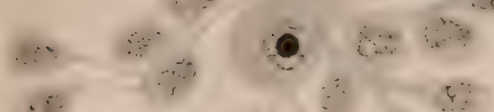
$4 x-\cos ^{4}$ $4 x^{2}+a^{8}$
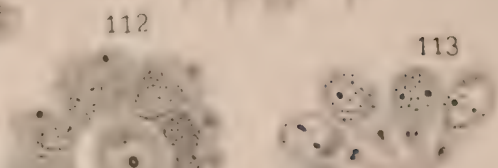

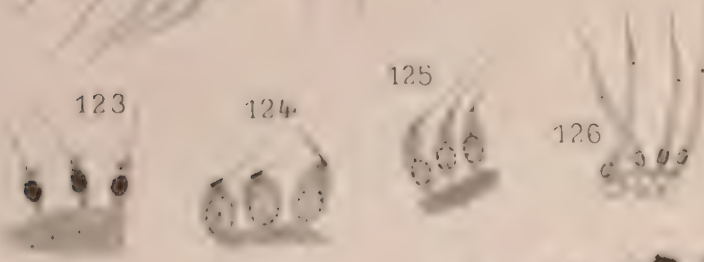
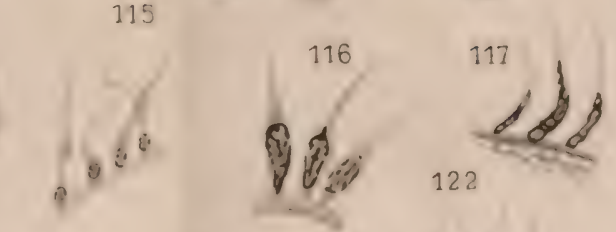

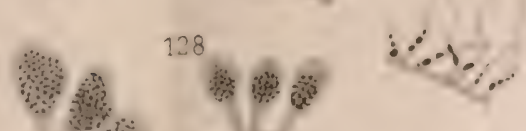

if 118 is
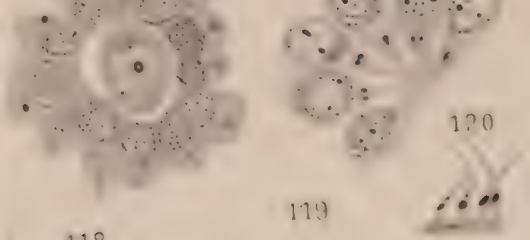

(n)
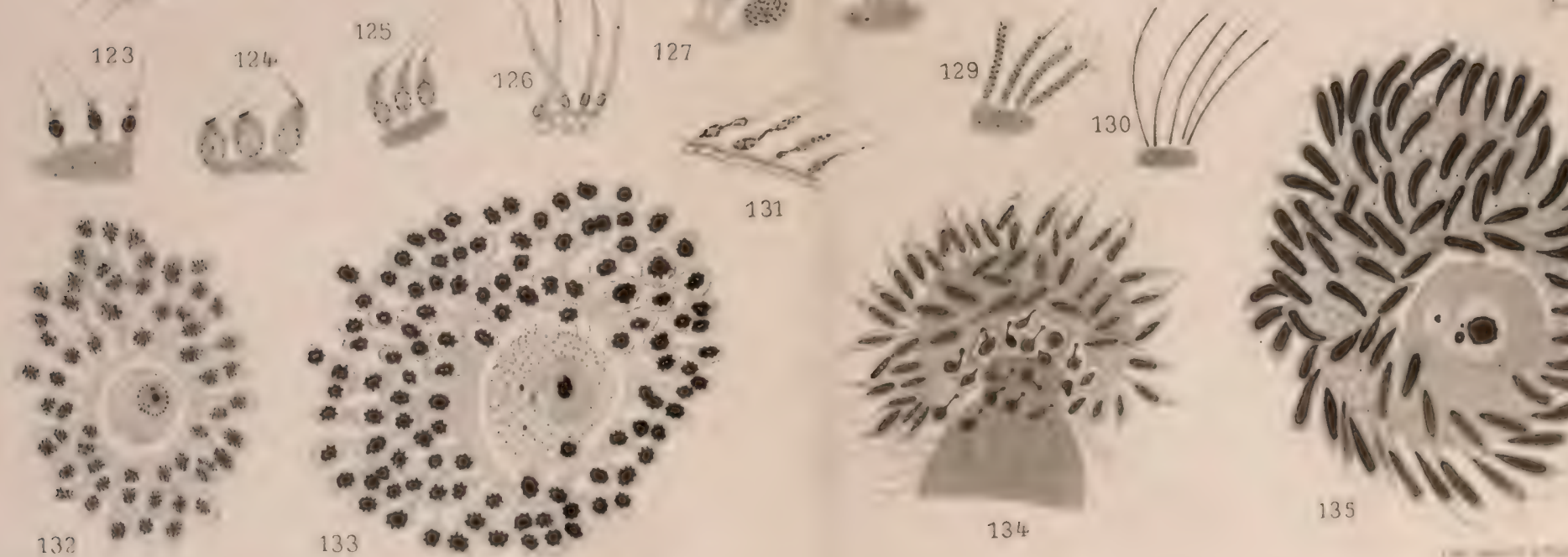

35

Ait. Hease dicl 


Arch de Zool. Expp. et Gén?

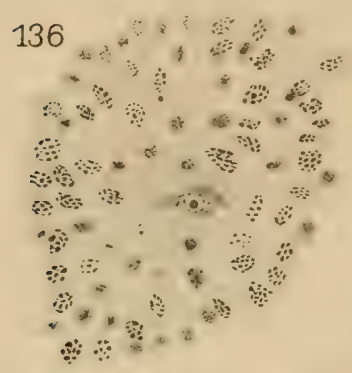

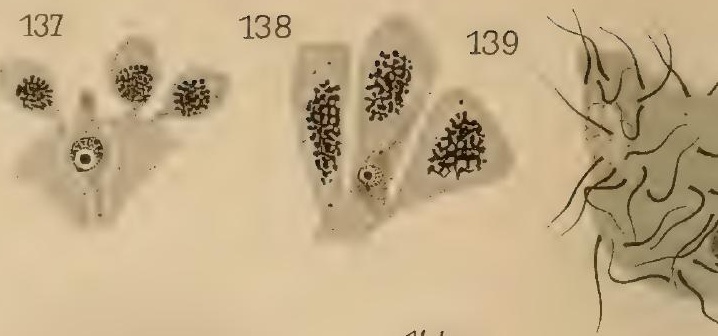

143

151

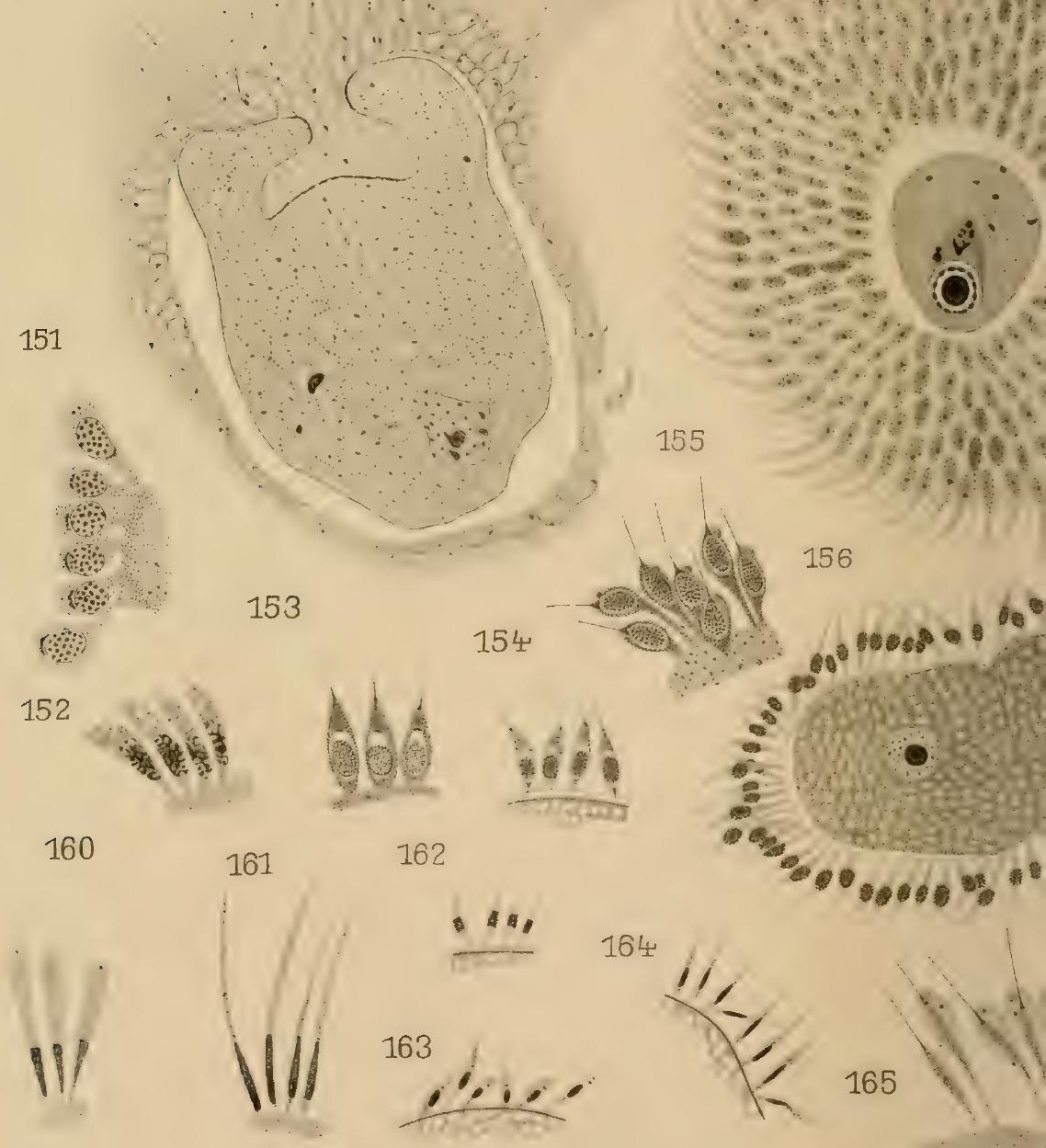


$\therefore$. . ......

$$
\begin{aligned}
& 136 \ldots 137
\end{aligned}
$$

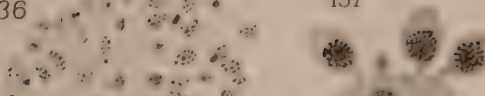

$$
\begin{aligned}
& a^{\infty}-0-\infty \\
& \because \div ;= \\
& \text { a }
\end{aligned}
$$

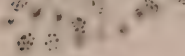

143$$
143
$$

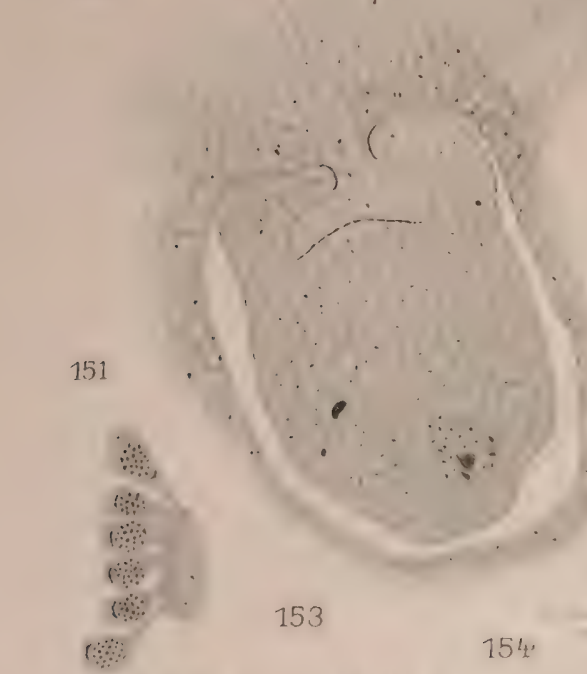

\section{2 \\ (w) Me wa}

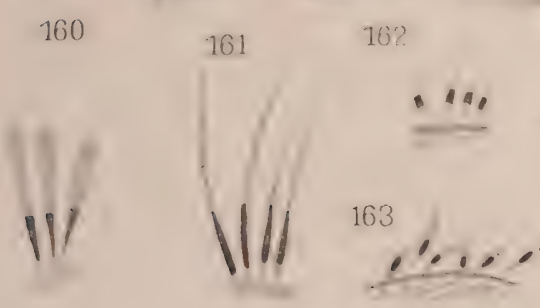

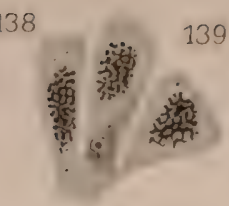

114

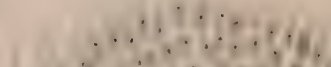

$\therefore \because y)$

$\therefore 3, \ldots$ $\cdots, \ldots, y, \ldots, \cdots$

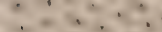

$\therefore \div \div$

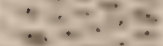

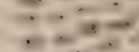

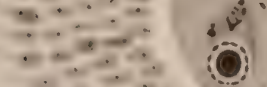
$-2.2 .10 \times 8$

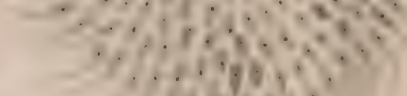

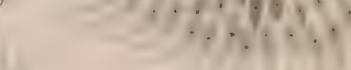

\section{-} $+25$

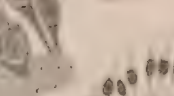
(2) $(2)=5$

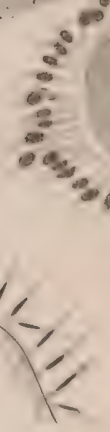

165

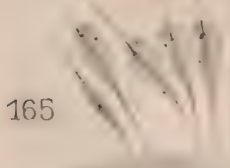

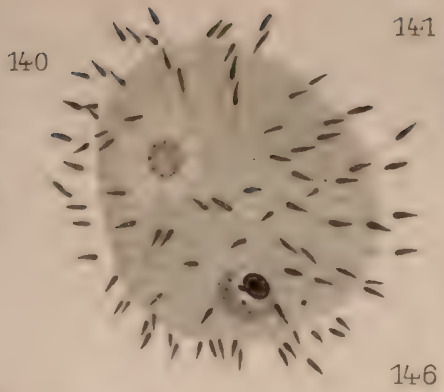

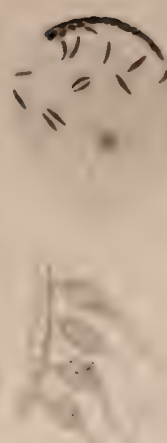

147

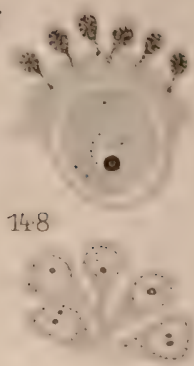

14.9

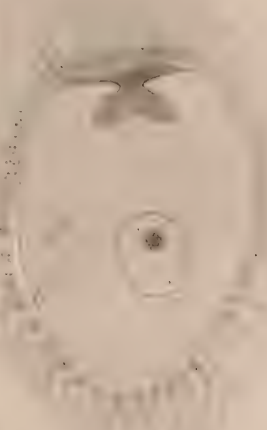

150
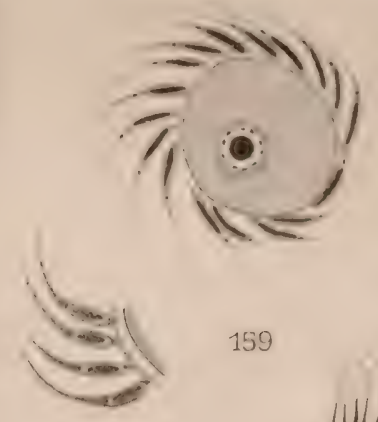

159 $\rightarrow$
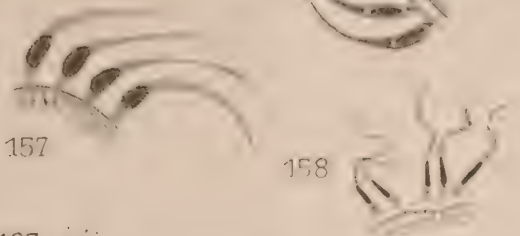

IIIIII

170

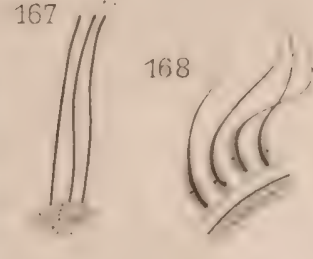



Arch de Zool. Exp. et Gén?

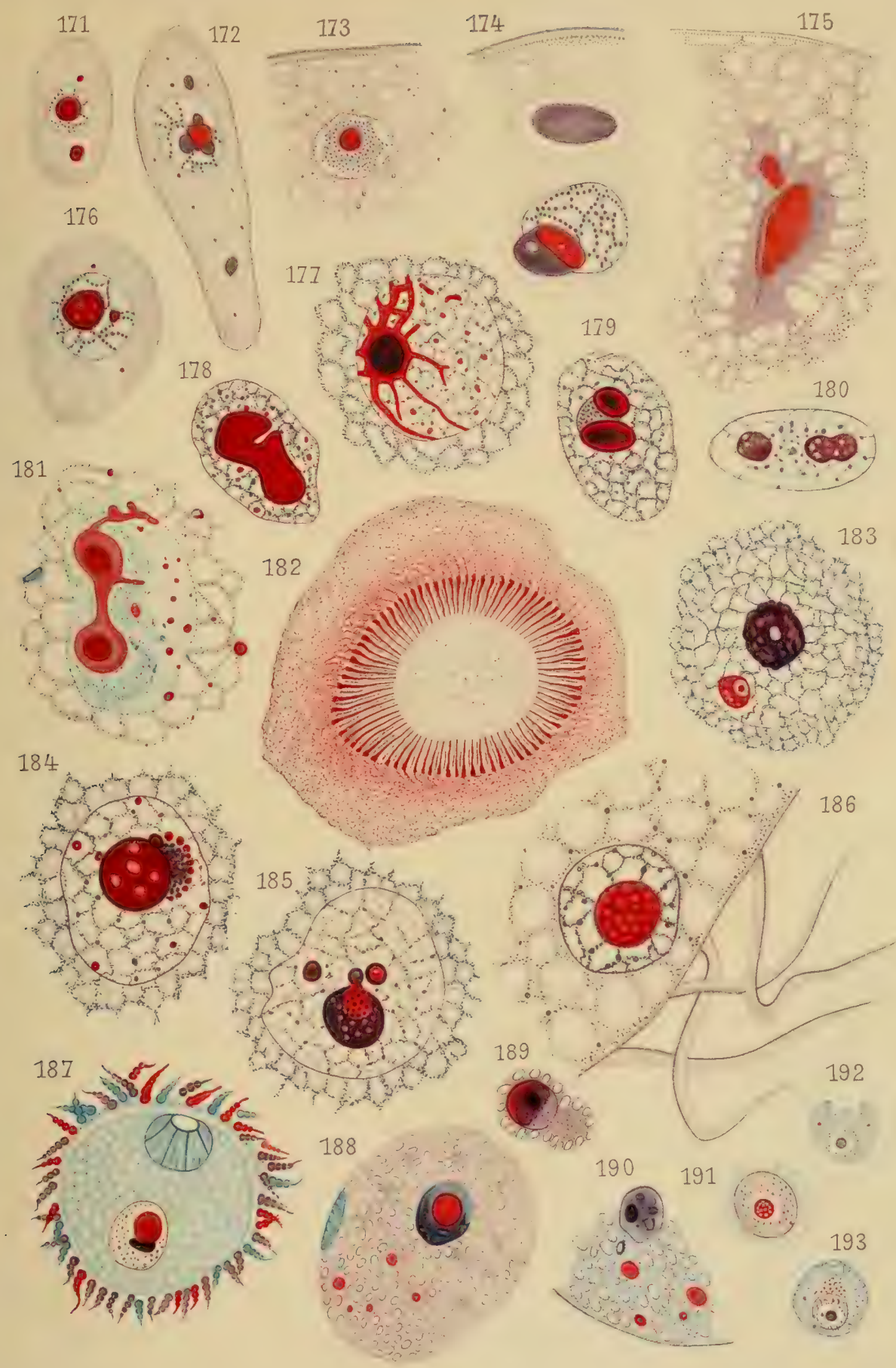


$\psi$ 


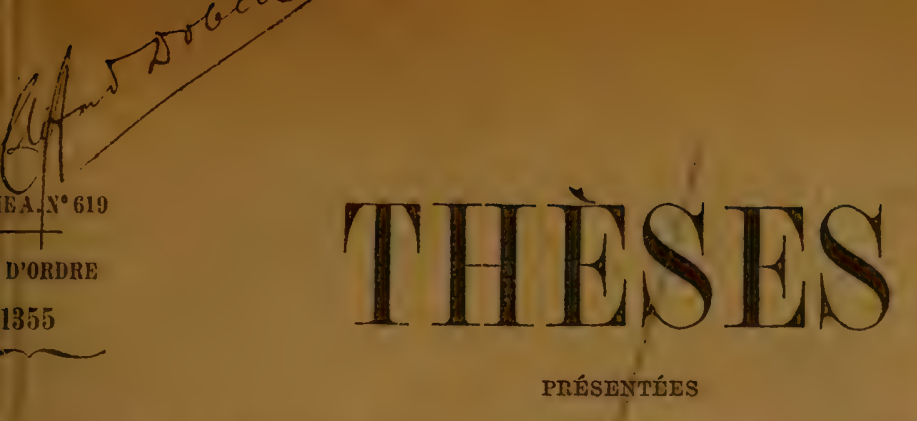

\section{A LA FACULTÉ DES SGIENCES DE L'UNIVERSTTÉ DE PARIS \\ POUR OBTENIR}

LE GRADE DE DOCTEUR ÈS SCIENCES NATURELLES

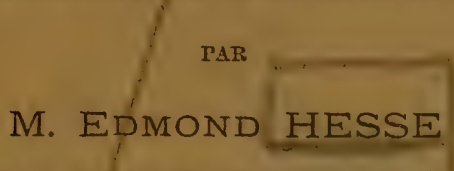

PREPARATEUR DE ZOOLOCIE A LA FACULTG DES SCIENCES DE L'UNIVERSITE DE GRENOBLE

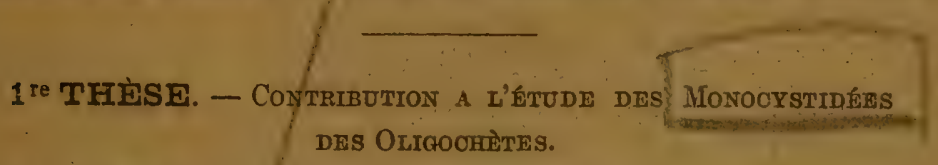

$2^{\text {me }}$ THESE. - PROPOSTIONS DONNEES PAR LA FACULTE.

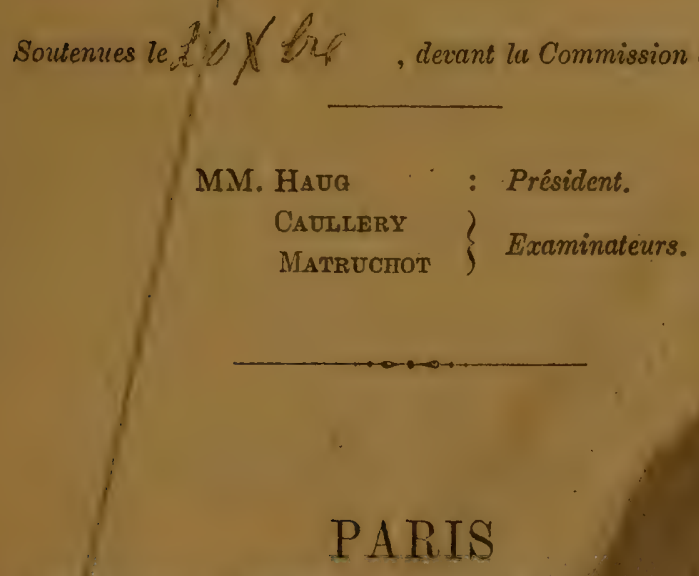

ĹDITIOA DES AROHIVES DE ZOOLOGIS F

LIBRAIRIE A. SCF

3, Place de la So: 





SMITHSONIAN INSTITUTION LIBRARIES

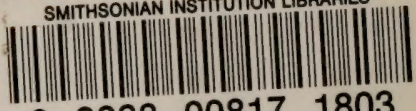

$3908800817 \quad 1803$ 